\title{
Ein Beitrag zur einheitlichen Modellierung und durchgängigen Nutzung fertigungstechnologischen Wissens im Produktentwicklungsprozess
}

\author{
Vom Fachbereich Maschinenbau \\ der Technischen Universität Darmstadt \\ zur Erlangung des akademischen Grades \\ Doktor-Ingenieur (Dr.-Ing.) \\ genehmigte \\ DISSERTATION \\ vorgelegt von \\ Michael Roos
}

aus Marktheidenfeld

Erstgutachter: Prof. Dr. h. c. Dr. h. c. Dr.-Ing. Herbert Birkhofer Zweitgutachter: Prof. Dr.-Ing. Dipl.-Wirtsch.-Ing. Peter Groche 
Roos, Michael: Ein Beitrag zur einheitlichen Modellierung und durchgängigen Nutzung fertigungstechnologischen Wissens im Produktentwicklungsprozess

Darmstadt, Technische Universität Darmstadt, Jahr der Veröffentlichung der Dissertation auf TUprints: 2018

URN: urn:nbn:de:tuda-tuprints-71876

Tag der mündlichen Prüfung: 24.10.2017

Veröffentlicht mit den vom Gesetz vorgesehenen Nutzungsrechten gemäß UrhG 


\section{Vorwort}

Die Entwicklung von Produkten und die gleichzeitige intensive Auseinandersetzung mit geeigneten Fertigungsprozessen ist eine Herausforderung, der sich bereits viele namhafte Ingenieure gestellt haben. Als einer der herausragenden $\mathrm{Na}-$ men ist auch Henry Ford (1863-1947) zu nennen. Er erkannte die Relevanz einer aufeinander abgestimmten Produktentwicklung und Fertigung ohne die er Produkte wie das „Modell T“ kaum hätte erfolgreich umsetzen können. Doch auch er bemerkte einst: „Es gibt mehr Leute, die kapitulieren, als solche, die scheitern." [Henry Ford] Ganz im Sinne dieser Aussage ist auch diese Dissertation nicht nur das Resultat jahrelanger Forschung zum Thema einer fertigungsintegrierenden Produktentwicklung, sondern vielmehr auch das Ergebnis eines ständigen Ringens mit Hürden, Rückschlägen und den eigenen Bedenken. Aus diesem Grund waren vor allem die Impulse aus unzähligen Gesprächen und Diskussionen von großer Bedeutung für den Fortschritt meiner Arbeit. Den betreffenden Personen möchte ich an dieser Stelle meinen ganz persönlichen Dank aussprechen.

In erster Linie gilt mein Dank meinem Doktorvater, Herrn Prof. Dr. h. c. Dr. h. c. Dr.-Ing. Herbert Birkhofer. Er hat das erste Referat dieser Arbeit mit großem Engagement übernommen, obwohl er die aktive Leitung des Fachgebiets Produktentwicklung und Maschinenelemente der Technischen Universität Darmstadt (pmd) bereits seit einigen Jahren an seine Nachfolger abgegeben hat. Seine Ideen und Anmerkungen aber auch seine Kritik lieferten mir stets äußert wertvolle Denkanstöße. Gerne blicke ich auf unsere gemeinsamen Gespräche zurück. Darüber hinaus bedanke ich mich ganz herzlich bei Herrn Prof. Dr.-Ing. Dipl.-Wirtsch.-Ing. Peter Groche. Nicht nur als Korreferent dieser Arbeit hat er mich maßgeblich unterstützt. Auch als Sprecher des Sonderforschungsbereichs 666 und zwischenzeitlicher Leiter des Fachgebiets pmd hat er meine Forschung über die letzten Jahre stetig begleitet und vorangetrieben.

Mein besonderer Dank gilt Herrn Dr.-Ing. Dipl.-Wirtsch.-Ing. Sebastian Gramlich, dessen Vorarbeiten und Ideen das Fundament darstellen, auf dem ich diese Arbeit aufbauen konnte. Seine Denkansätze haben mich und die Inhalte dieser Arbeit stark geprägt. Seiner strukturierten Arbeitsweise gilt auch heute noch meine größte Anerkennung. In gleichem Zuge möchte ich auch Herrn Christian Wagner danken, der mir über die vergangenen Jahre hinweg einer der scharfsinnigsten Diskussionspartner war, aber ebenso auch eine der wichtigsten Stützen während 
meiner Arbeit. Darüber hinaus gebührt auch den übrigen Kollegen des Fachgebiets pmd mein Dank für die stets kollegiale Zusammenarbeit und die vielen fachlichen Gespräche die wir gemeinsam geführt haben.

Zudem möchte ich Herrn Jannik Reichwein, Herrn Victor Listmann, Herrn Matthias Schaschek, Frau Heike Gute, Herrn Daniel Dieter und Herrn Martin Gowik danken, die mich insbesondere während der finalen Phase meines Forschungsvorhabens intensiv unterstützt haben. Ich habe sie allesamt über die gemeinsamen Jahre hinweg sehr schätzen gelernt.

Zuletzt gilt mein Dank auch der Deutschen Forschungsgemeinschaft (DFG), deren Förderung es mir ermöglicht hat, mich konsequent mit den fachlichen Schwerpunkten meiner Arbeit zu befassen.

Roßdorf, 10.01.2018

Michael Roos 


\section{Inhaltsverzeichnis}

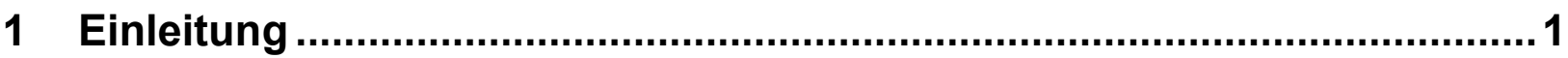

1.1 Bedeutung der Fertigung für die Entwicklung von Produkten ................ 1

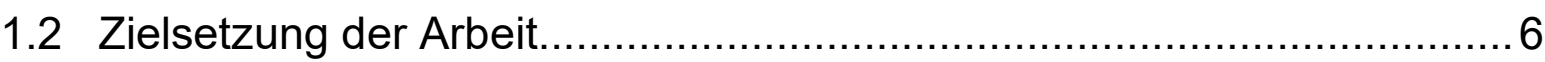

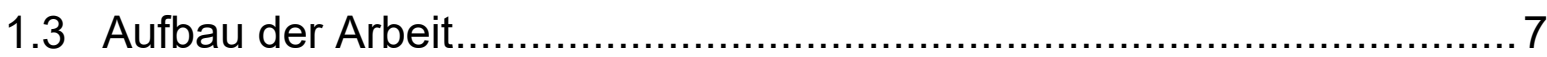

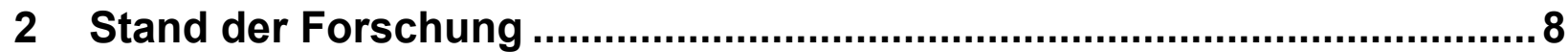

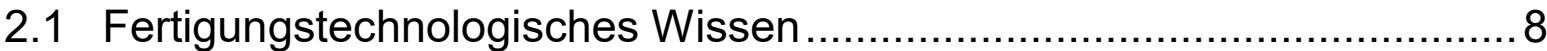

2.2 Dokumentationsformen für fertigungstechnologisches Wissen im Rahmen der Produktentwicklung

2.3 Methoden und Werkzeuge zur Aufarbeitung und Nutzung von fertigungstechnologischem Wissen in der Produktentwicklung

2.4 Modellbasierte Methodiken zur Berücksichtigung der Fertigung im Kontext der Produktentwicklung ................................................... 17

2.4.1 Modellierung technischer Produkte ...................................... 17

2.4.2 Modellierung technischer Prozesse ….................................27

2.4.3 Modellierung des Produktlebenslaufs .................................... 37

2.4.4 Integrale Ansätze zur Berücksichtigung der Fertigung während

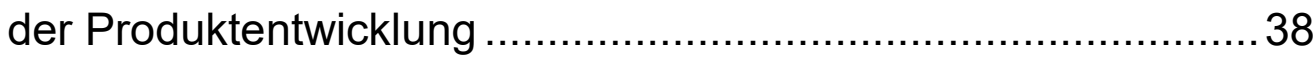

2.5 Generelles Bereitstellen und Nutzen von Wissen durch Wissensmanagementsysteme

2.6 Fazit - Defizite hinsichtlich der systematischen Entwicklung fertigungsintegrierender Produktlösungen

3 Idee einer fertigungsintegrierenden Entwicklungsmethodik

4 Standardisierung fertigungstechnologischen Wissens für die Produktentwicklung

4.1 Verfahren und fertigungstechnologisches Wissen ...........................47

4.2 Konkretisierungsebenen der Prozessmodellierung ............................50

4.2.1 Modellierung von Prozessen auf Basis der Zustandsänderung. 50

4.2.2 Modellierung von Verfahren auf Basis physikalischer Effekte ...54

4.2.3 Modellierung von Verfahren auf Basis des Verfahrensprinzips .56

4.3 Vorgehen zum Konkretisieren technischer Produkte im Kontext des Einsatzprozesses und der Produktfunktion ......................................67

4.4 Fazit - Abbildung fertigungstechnologischen Wissens ......................74 
5 Ansatz zum Entwickeln fertigungsintegrierender Produktlösungen .....75

5.1 Idee - Modellübergreifende Verarbeitung fertigungstechnologischen

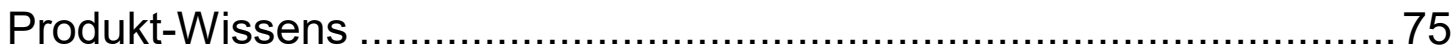

5.2 Antizipieren technischer Prozesse - Kernelement einer fertigungsintegrierenden Entwicklungsmethodik ..............................77

5.2.1 Grundgedanke des Antizipierens technischer Prozesse ............77

5.2.2 Antizipieren von Fertigungsprozessen .................................. 80

5.3 Vorgehensmodell zum Entwickeln fertigungsintegrierender

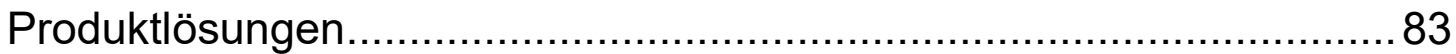

5.4 Fertigungsintegrierende Entwicklungsmethodik ................................ 85

5.5 Design Pattern Matrix - Werkzeug zur Entwicklung fertigungsintegrierender Produktlösungen ......................................... 88

5.5.1 Fertigungsintegrierende Design Patterns ................................ 88

5.5.2 Erarbeiten von Design Patterns - Design Pattern Matrix........... 90

5.5.3 Process Integrated Design Guidelines ...................................... 95

5.6 Fazit - Modellbasierte Entwicklungsmethodik.................................97

6 Evaluierung der fertigungsintegrierenden Entwicklungsmethodik .......99

6.1 Beispiel innenhochdruckumgeformter Fahrradrahmen ......................99

6.1.1 Antizipieren des Innenhochdruckumformens ......................... 101

6.1.2 Aufbereiten der Ergebnisse aus dem Antizipieren .................. 102

6.1.3 Ergreifen projektspezifischer Maßnahmen.............................109

6.2 Mehrfaches Antizipieren am Beispiel spaltprofilierter Deckenschienen

6.2.1 Antizipieren des Fügens von Schnappverbindungen .............. 113

6.2.2 Aufbereiten der Ergebnisse aus dem Antizipieren .................. 115

6.2.3 Ergreifen projektspezifischer Maßnahmen............................116

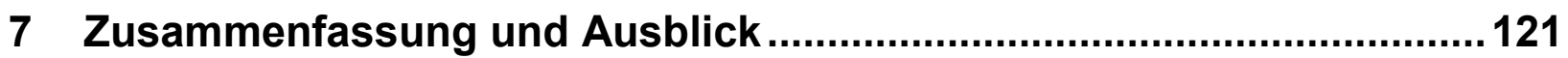

Anhang A - Design Pattern Matrix und Punktbewertung für das Beispiel innenhochdruckumgeformter Fahrradrahmen.....................................123

Anhang B - Beispiele für die Anwendung des VP-Modells .......................129

Anhang C - Process Integrated Design Guidelines ..................................135 
Anhang D - Übersicht über die Arbeitsschritte der fertigungsintegrierenden

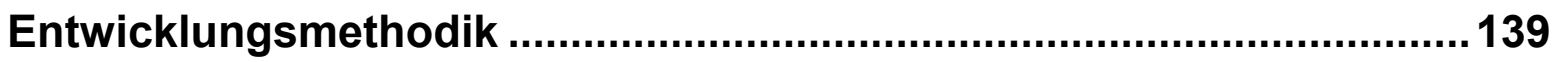

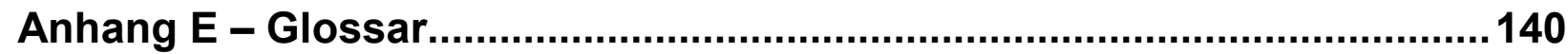

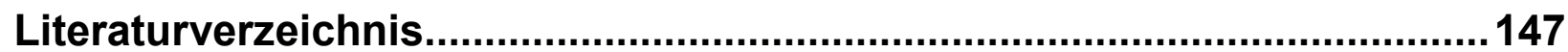

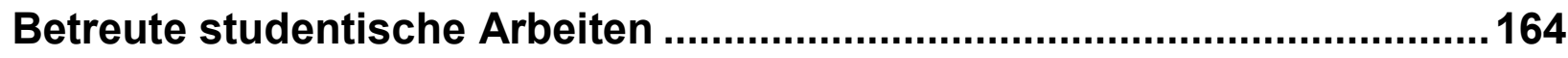

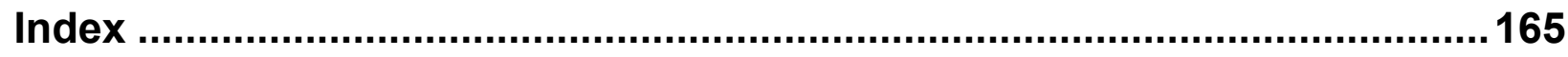




\section{Abkürzungsverzeichnis}

AZ

Bsp.

CAD

CPM

DfA

DfM

DfMA

DIN

DP

DPM

Eig

EZ

FE

Fkt

GE

GPPE

HSC

$\mathrm{IHU}$

PDD

PhEff

PIDG

PzEig

Spabi

Spapro

TF

UFG

VDI

VP

Wapro

WE

WP
Anfangszustand

Beispiel

Computer Aided Design

Characteristics-Properties Modelling

Design for Assembly

Design for Manufacture

Design for Manufacture and Assembly

Deutsches Institut für Normung

Design Pattern

Design Pattern Matrix

Eigenschaft

Endzustand

Finite Element

Funktion

Gestaltelement

Ganzheitliche Produkt- und Prozessentwicklung

High Speed Cutting

Innenhochdruckumformen

Property-Driven-Development

Physikalischer Effekt

Process Integrated Design Guideline

Prozesseigenschaft

Spaltbiegen

Spaltprofilieren

Teilfunktion

Ultra Fine Grained

Verein Deutscher Ingenieure

Verfahrensprinzip

Walzprofilieren

Wirkelement

Wirkprinzip 


\section{Kurzfassung}

Unter dem Begriff des Design for Manufacture werden eine Vielzahl an Produktentwicklungsansätzen zusammengefasst, die den Entwickler vor allem im Hinblick auf das Sicherstellen der Herstellbarkeit technischer Produkte unterstützen. Es mangelt jedoch an Ansätzen, die die noch ungenutzten Möglichkeiten einer Fertigungstechnologie zur Realisierung oder Verbesserung des technischen Produkts aufzeigen. Diese ungenutzten Möglichkeiten können der Ausgangspunkt für neuartige fertigungsintegrierende Produktlösungen sein, die sich z. B. durch eine verbesserte Funktionserfüllung, einen erhöhten Leichtbaugrad oder auch eine erhöhte Lebensdauer auszeichnen.

Im Rahmen dieser Arbeit wird der Grundstock für die Systematisierung einer fertigungsintegrierenden Produktentwicklung gelegt, mittels der die noch ungenutzten Möglichkeiten einer betrachteten Fertigungstechnologie systematisch erschlossen werden können. Dazu wird eine Methodik vorgestellt, die den Produktentwickler gezielt bei der Aufbereitung vorhandenen fertigungstechnologischen Wissens unterstützt. Durch die Modellierung technischer Prozesse mittels Eigenschaften kann fertigungstechnologisches Wissen einheitlich abgebildet und dokumentiert werden. Der Fokus der Modellierung richtet sich vor allem auf die Abbildung von Wirkzusammenhängen im Kontext eines gewählten Fertigungsverfahrens. Sie geben Aufschluss über das den Fertigungsprozessen zugrunde liegende Verfahrensprinzip. Das so abgebildete fertigungstechnologische Wissen wird im Rahmen eines frühzeitig im Produktentwicklungsprozess durchgeführten Antizipierens von Fertigungsprozessen umfassend analysiert. Aufeinander abgestimmte Produkt- und Prozessmodelle bilden darüber hinaus die Basis für die weitere Aufbereitung des fertigungstechnologischen Wissens mit Blick auf die Produktentwicklung. Die durch das Antizipieren erfassten Eigenschaften und Eigenschaftsrelationen dienen der anschließenden Erarbeitung fertigungsintegrierender Produktlösungen. Durch Zuhilfenahme geeigneter Werkzeuge, wie der Design Pattern Matrix, wird der Produktentwickler bei deren Erarbeitung gezielt unterstützt. Die Anwendung der vorgestellten Methodik anhand zweier Fallbeispiele verdeutlicht den Mehrwert, den die Fertigung für die Realisierung der betrachteten technischen Produkte bietet. Dieser Mehrwert zeigt sich an den Beispielen u. a. durch zusätzlich integrierte Funktionen, eine erhöhte Bauteilsteifigkeit oder eine Produktgestalt, die Einsatzund Fügeprozessen gleichermaßen gerecht wird. 



\section{Einleitung}

Für Produktentwicklungsprojekte existiert eine Vielzahl unterschiedlicher Treiber, die alle einen maßgeblichen Einfluss auf die Lösungsfindung und -konkretisierung haben. Fertigungstechnologien sind einer dieser Treiber. Sie sind nicht nur für die Realisierung der technischen Produkte verantwortlich, sondern stellen auch Möglichkeiten bereit, auf deren Basis neuartige Produktlösungen konzipiert und umgesetzt werden können. Es ist daher unabdingbar, dem Produktentwickler ${ }^{1}$ fertigungstechnologisches Wissen zur Verfügung zu stellen, um inn im Hinblick auf die Lösungsfindung und -konkretisierung zu unterstützen. Fertigungstechnologisches Wissen gehört zusammen mit weiterem natur- und ingenieurwissenschaftlichen Wissen zu den wichtigsten Grundlagen für die Entwicklung technischer Produkte. ${ }^{2}$

Das fertigungstechnologische Wissen beinhaltet allgemeingültige ${ }^{3}$ Aussagen, die u. a. aus Versuchen, analytischen Rechnungen, Beobachtungen oder Simulationen abgeleitet wurden und deren Relevanz für das Verständnis der Fertigungstechnologie belegt werden konnte. ${ }^{4}$

Das systematische Anwenden des fertigungstechnologischen Wissens im Rahmen der Produktentwicklung und das umfassende Ausschöpfen fertigungstechnologischer Potenziale für neuartige Produktlösungen gestaltet sich nicht immer leicht und bedarf daher einer gezielten methodischen Unterstützung.

\subsection{Bedeutung der Fertigung für die Entwicklung von Produkten}

Die Produktentwicklung und die Fertigung ${ }^{5}$ repräsentieren zwei zentrale Bereiche innerhalb von Unternehmen, die beide einen großen Einfluss auf die Realisierung

\footnotetext{
${ }^{1}$ Bei PAHL findet sich der Begriff Konstrukteur synonym für Entwicklungs- und Konstruktionsingenieure. (Vgl. Pahl et al. (2007), S. 1.)

${ }^{2}$ Vgl. Pahl et al. (2007), S. 1.

${ }^{3}$ Die Allgemeingültigkeit einer Aussage zu einer Fertigungstechnologie kann erst dann sichergestellt werden, wenn der Nachweis erbracht wird, dass die Aussage auch unter geänderten Rand-/ Versuchs-/ Simulationsbedingungen getroffen werden kann.

${ }^{4}$ Vgl. Roos et al. (2014), S. 1855. Auch in Flach (1994), S. $133 \mathrm{ff}$.

${ }^{5}$ Die Fertigung dient im Allgemeinen der Herstellung geometrisch bestimmter materieller Güter in diskreten Prozessen. (Vgl. Dangelmaier (2003), S. 50; DIN 8580 (2003), S. 4; Westkämper (2006), S. 24.) In diesem Kontext wird häufig auch der Begriff Produktion verwendet. Produktion wird als Überbegriff über alle technischen und organisatorischen Prozesse zur Herstellung, Erhalt und Recycling von Produkten und damit auch über Fertigungsprozesse verstanden. (Vgl. Westkämper (2006), S. 24.)
} 
erfolgreicher Produkte haben. Erfolgreiche Produkte erfordern auf der einen Seite die Bereitstellung eines hohen Kundennutzens. Auf der anderen Seite ist eine möglichst hohe Effizienz und Effektivität bei der Realisierung der Produkte anzustreben. Dazu müssen wesentliche Anforderungen in Bezug auf Qualität, Flexibilität, Kosten und Durchlaufzeiten eingehalten werden. ${ }^{6}$ Das Erreichen dieses Ziels wird vor allem durch die Vielzahl an existierenden Fertigungstechnologien erschwert. ${ }^{7}$ Jede Fertigungstechnologie zeichnet sich durch spezifische Charakteristika aus, die nicht nur den Fertigungsprozess, sondern auch die realisierbaren Produkte betreffen. Anhand des einfachen Beispiels eines Biegebalkens lässt sich dieser Einfluss verdeutlichen (siehe Abbildung 1-1).

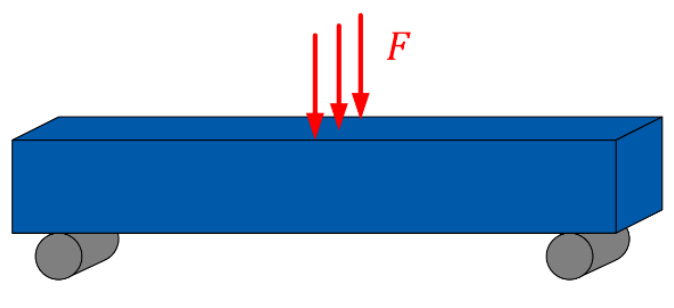

Funktion des

Biegebalkens:

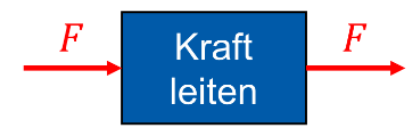

A)

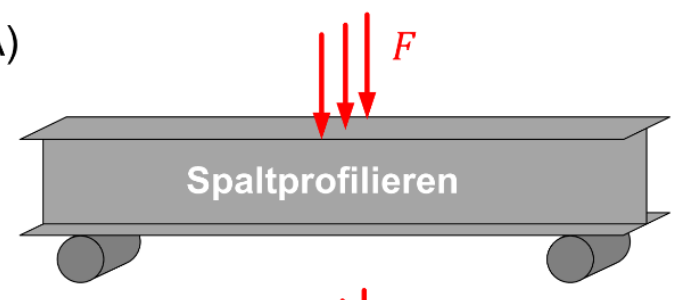

B)

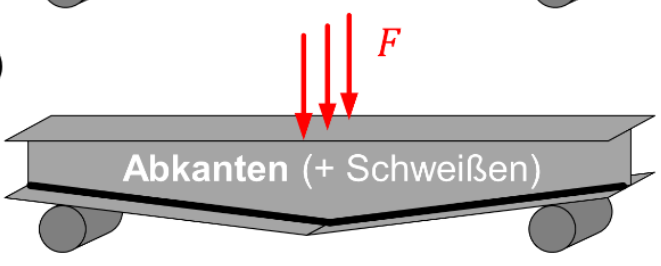

C)

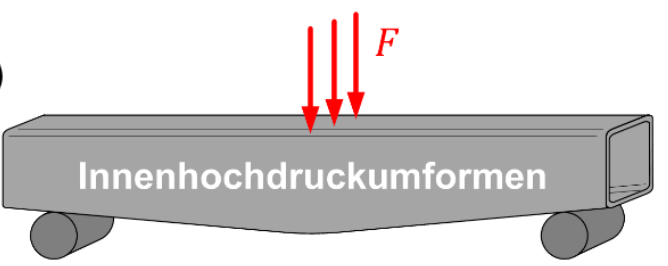

Abbildung 1-1: Ausführungsformen A, B und C eines Biegebalkens unter Berücksichtigung fertigungstechnologischer Charakteristika

Die grundsätzliche Funktion des Biegebalkens ist einfach: Ausgehend vom Krafteinleitungspunkt soll die Querkraft $F$ auf möglichst direktem Weg in die Lager geleitet werden. Für die Realisierung dieser Funktion sind unterschiedliche Ausführungsformen denkbar. Drei dieser Ausführungsformen sind in Abbildung 1-1 dargestellt. Ausführungsform A des Biegebalkens wird durch Verwendung der Fertigungstechnologie Spaltprofilieren ${ }^{8}$ in Kombination mit Walzprofilieren der spaltprofilierten Flansche ${ }^{9}$ erzeugt (siehe Abbildung 1-1). Durch das Walzprofilieren der

\footnotetext{
${ }^{6} \mathrm{Vgl}$. Tangen (2005), S. $40 \mathrm{ff}$.

${ }^{7}$ Vgl. DIN 8580 (2003).

${ }^{8} \mathrm{Vgl}$. Jöckel (2005).

${ }^{9}$ Vgl. Neuwirth et al. (2017), S. $52 \mathrm{ff}$.
} 
Flansche werden vollständig ebene Gurtbereiche realisiert. Die in das Profil eingebrachten Verzweigungen in Form der spaltprofilierten Flansche ermöglichen es, gezielt Material in die stark biegebeanspruchten Randbereiche des Profils zu legen. Auf Fügeoperationen kann dabei komplett verzichtet werden. Dies hat den Vorteil, dass das Material in den Verzweigungsbereichen keine Gefügeänderungen aufweist, wie sie charakteristischerweise infolge von Schweißoperationen auftreten. Zwar ist die spaltprofilierte Querschnittgeometrie beanspruchungsgerechter als ein Balken mit Vollquerschnitt. Dennoch besteht weiteres Optimierungspotenzial in Bezug auf die Anpassung der Geometrie an die Schnittkraftverläufe über der Balkenlänge. Aufgrund der aufwendigen Werkzeugherstellung und der damit verbundenen Kosten ist die spaltprofilierte Ausführungsform des Biegebalkens vor allem für große Stückzahlen geeignet.

Eine abgekantete geschweißte Ausführungsform B des Biegebalkens (siehe Abbildung 1-1) führt bei gleicher maximaler Bauteilhöhe zu einer deutlich beanspruchungsgerechteren Gestalt. Zwar kann die offene Profilgeometrie ebenso wie die spaltprofilierte Ausführungsform nur geringe Torsionsbelastungen aufnehmen, falls diese überlagert auftreten sollten. Dennoch wird durch eine geschickte Anpassung des Profilquerschnitts an den Biegemomentenverlauf ein annähernd gleichmäßiger Biegespannungsverlauf über der Balkenlänge erreicht. Auf diese Weise können unnötige Materialanhäufungen vermieden werden, wodurch nicht nur ein erhöhter Leichtbaugrad, sondern auch eine Fertigung mit geringerem Materialaufwand möglich wird. Durch die Verwendung mehrerer Einzelteile, die anschließend einzeln geschweißt werden, erhöht sich der zeitliche Aufwand für die Fertigung und Montage. Bei geringen Stückzahlen fallen die Kosten im Vergleich zur spaltprofilierten Lösung dennoch deutlich geringer aus, da keine kostenintensiven Werkzeuge benötigt werden. ${ }^{10}$

Der Einsatz der Fertigungstechnologie Innenhochdruckumformen (IHU) ${ }^{11}$ ermöglicht die Ausführung $\mathrm{C}$ des Biegebalkens, die als Hohlkammerprofil mit einer ebenfalls an den Momentenverlauf angepassten Querschnittgeometrie ausgelegt ist (siehe Abbildung 1-1). Aufgrund des geschlossenen Querschnitts zeichnet sich der Balken zusätzlich durch eine hohe Torsionssteifigkeit gegenüber offenen Querschnittgeometrien aus. Der Einsatz von Innenhochdruckumformen ermög-

\footnotetext{
${ }^{10} \mathrm{Vgl}$. Matek et al. (2011), S. 114.

${ }^{11}$ Vgl. Neugebauer (2007).
} 
licht zudem die Herstellung großer Stückzahlen bei kurzen Durchlaufzeiten. Bedingt durch die hohen Werkzeugkosten und des hohen Einrichtungsaufwands und der damit verbunden geringen Flexibilität der Fertigung eignet sich diese Ausführungsform des Biegebalkens kaum für die Herstellung von Einzelbauteilen.

Die dargestellten Beispiele zeigen, dass die Wahl der Fertigungstechnologie starken Einfluss auf die Möglichkeiten hat, die sich für die Umsetzung des technischen Produkts z. B. im Hinblick auf Beanspruchungsgerechtheit oder Leichtbau und damit dessen Funktionserfüllung ergeben. Umgekehrt beeinflusst die Produktgestalt auch die Möglichkeiten der Fertigung in Bezug auf Aufwand, Zeit und Kosten.

Innovative Fertigungstechnologien bieten neben den Möglichkeiten zur Realisierung definierter Produktgeometrien gleichzeitig Möglichkeiten, die mechanischen und werkstofflichen Eigenschaften der Bauteile gezielt zu beeinflussen. Spaltprofilierte Bauteile weisen so z. B. eine erhöhte Härte (siehe Abbildung 1-2), Festigkeit und Wälzfestigkeit im Flanschbereich auf. Die Nutzbarmachung dieser mechanischen und werkstofflichen Eigenschaften in Kombination mit den Möglichkeiten der Formgebung der Bauteile stellt für die Produktentwicklung eine zentrale Herausforderung dar. Jedoch bietet die Erschließung dieser Eigenschaften zugleich ein erhebliches Innovationspotenzial im Hinblick auf die Funktionserfüllung des Produkts.

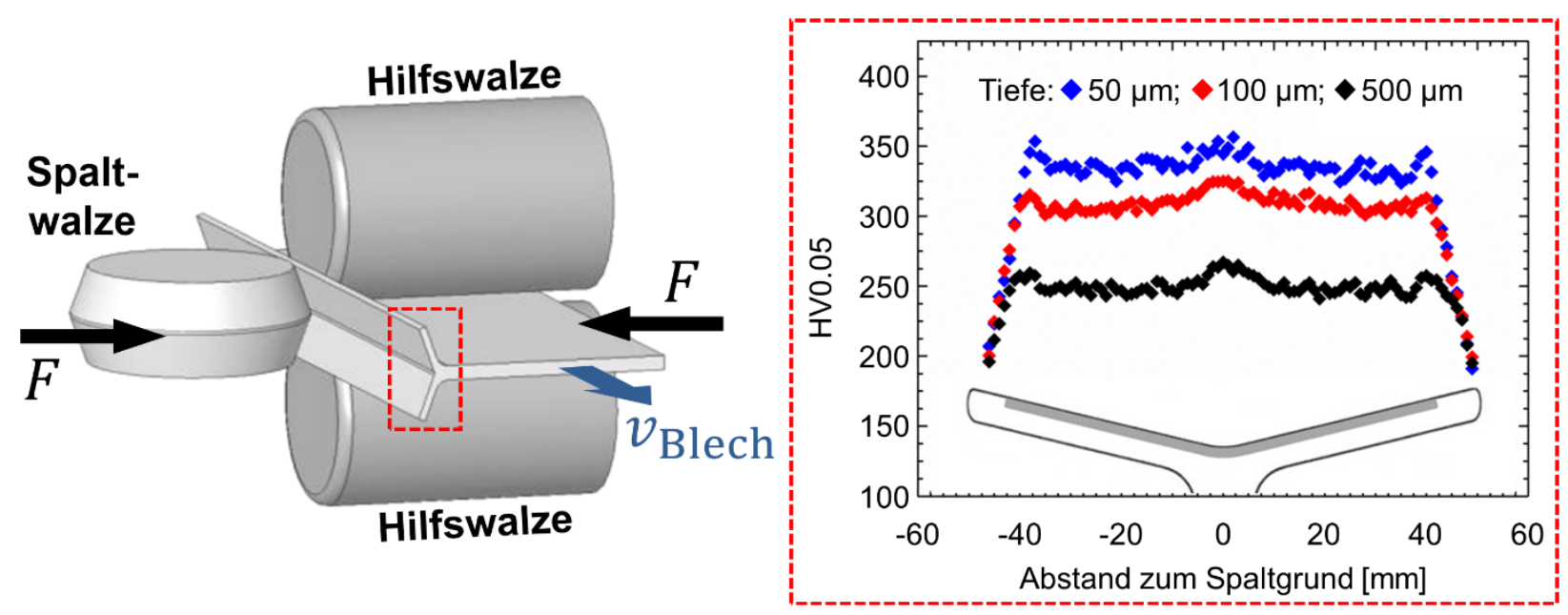

Abbildung 1-2: Aufbau des Werkzeugsystems für das Spaltprofilieren von Blechbauteilen und Härteverlauf über den spaltprofilierten Flanschen ${ }^{12}$

12 In Anlehnung an Groche et al. (2010) und Ahmels et al. (2017), S. 111. 
Die hohe Wälzfestigkeit spaltprofilierter Bauteile im Bereich der Flansche bietet die Möglichkeit, diese als Profile in Wälzführungen einzusetzen. ${ }^{13}$ So können sie z. B. als Führungsprofil in einem spaltprofilierten Leichtkransystem verwendet werden (siehe Bereich A in Abbildung 1-3), welches Lasten tragen und bewegte Komponenten translatorisch führen soll. ${ }^{14}$
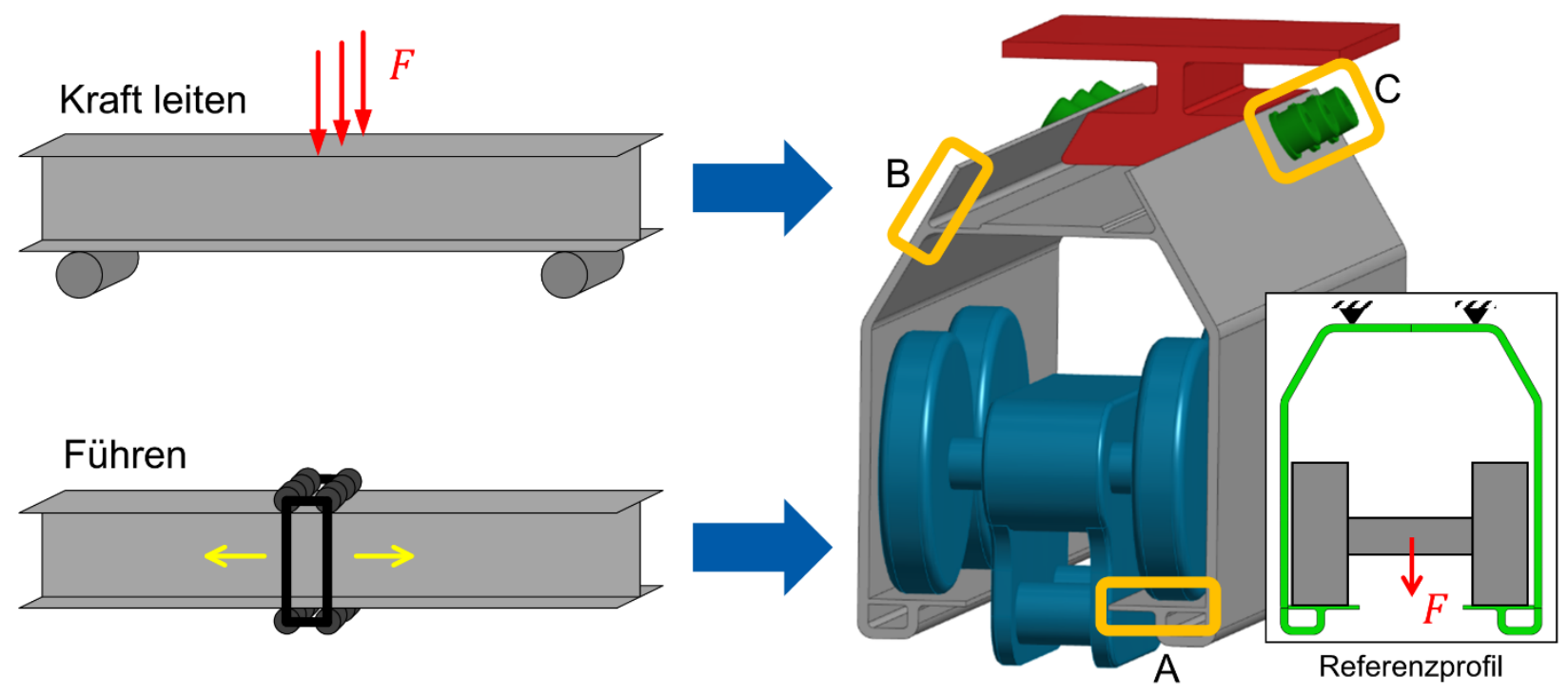

Führen
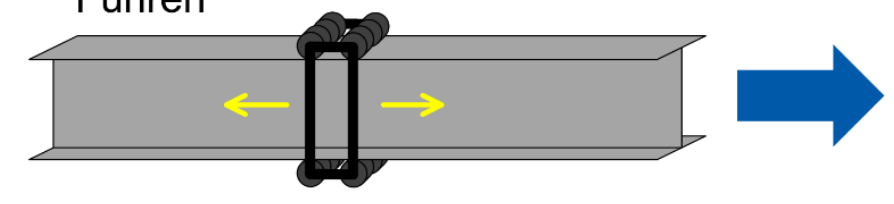

Referenzprofil

\section{Abbildung 1-3: Fertigungsintegrierende Produktlösung am Beispiel eines spaltprofilierten Leichtkransystems ${ }^{15}$}

Die Verwendung spaltprofilierter Flansche als tragende Elemente in hoch beanspruchten Bereichen des Führungsprofils (siehe Bereich B in Abbildung 1-3) führt zu einer um den Faktor 1,6 gesteigerten Lebensdauer der Führung im Vergleich zu einem Referenzprofil (siehe Referenzprofil in Abbildung 1-3), das die Möglichkeiten der Fertigungstechnologie nicht umfassend ausschöpft. Das gezielte Einbringen geometrischer Verzweigungen (siehe Bereich B in Abbildung 1-3) erhöht die Steifigkeit der Profilstruktur um den Faktor 1,4 im Vergleich zum Referenzprofil. Unter Ausnutzung der verminderten Kerbempfindlichkeit spaltprofilierter Flansche konnten Formelemente in das Profil integriert werden (siehe Bereich $\mathrm{C}$ in Abbildung 1-3), die als Verbindungselemente genutzt werden. ${ }^{16}$

\footnotetext{
${ }^{13} \mathrm{Vgl}$. Lommatzsch et al. (2011), S. 442.

${ }^{14} \mathrm{Vgl}$. Gramlich et al. (2015).

15 In Anlehnung an Gramlich et al. (2015), S. 8.

${ }^{16}$ Vgl. Gramlich et al. (2015), S. 9.
} 
Der Vergleich des spaltprofilierten Führungsprofils mit dem Referenzprofil zeigt deutlich, dass die gewählte Fertigungstechnologie konkrete Möglichkeiten zur Verbesserung der Funktionserfüllung des Produkts bietet. Bleiben diese Möglichkeiten ungenutzt, so ergeben sich daraus Potenziale für die Produktentwicklung. Eine besondere Rolle bei der Ausschöpfung dieser Fertigungspotenziale spielen die Bauteileigenschaften, die durch eine bestimmte Fertigungstechnologie in charakteristischer Weise erzeugt werden, wie z. B. die erhöhte Wälzfestigkeit spaltprofilierter Flansche. Deren nutzenbringende Integration in einen Bauteilentwurf führt letztlich zu fertigungsintegrierenden Produktlösungen. Dies bedingt jedoch umfassendes Wissen über die Fertigungstechnologie. Nur durch den gezielten Zugriff auf fertigungstechnologisches Wissen ist es möglich, geeignete Schlüsse über eine vorteilhafte Produktgestaltung zu ziehen. ${ }^{17}$ Aus dieser Tatsache heraus können fertigungsintegrierende Produktlösungen wie folgt definiert werden:

Fertigungsintegrierende Produktlösungen sind Lösungen, die nicht nur die Erwartungen der Stakeholder bestmöglich erfüllen, sondern auch einen erkennbaren Mehrwert in Bezug auf die Erfüllung der Produktfunktion sowie die Fertigung und Montage bieten, indem Fertigungspotenziale durch Zurückgreifen auf fertigungstechnologisches Wissen umfassend genutzt werden. ${ }^{18}$

\subsection{Zielsetzung der Arbeit}

Ziel dieser Arbeit ist es, ein systematisches Vorgehen bereitzustellen, mittels dessen es dem Produktentwickler möglich ist, die Potenziale einer Fertigungstechnologie bei der Entwicklung fertigungsintegrierender Produktlösungen umfassend auszuschöpfen. Der Produktentwickler soll hierzu durch geeignete Methoden und Werkzeuge unterstützt werden, die es ihm erlauben, während des Produktentwicklungsprozesses gezielt auf fertigungstechnologisches Wissen zurückzugreifen. Dies erfordert eine vereinheitlichte Modellwelt, in der das fertigungstechnologische Wissen abgebildet und auf seine für die Produktentwicklung relevanten Aussagen hin analysiert werden kann. Zudem soll gezeigt werden, welcher Nutzen sich durch Anwendung des systematischen Vorgehens anhand konkreter Beispiele generieren lässt.

\footnotetext{
${ }^{17}$ Vgl. Gramlich et al. (2015), S. 3 ff.

${ }^{18} \mathrm{Vgl}$. Roos et al. (2017a), S. 13.
} 


\subsection{Aufbau der Arbeit}

Ein Überblick über existierende Ansätze zur Speicherung und Verarbeitung fertigungstechnologischen Wissens wird in Kapitel 2 gegeben. Im Fokus stehen vor allem modellbasierte Ansätze, die mittels einer konsistenten Differenzierung technischer Produkte und Prozesse bestimmte Charakteristika von Fertigungstechnologien modellübergreifend verarbeiten können. Kapitel 3 reflektiert die vorhandenen Ansätze und legt den Grundgedanken einer neuartigen fertigungsintegrierenden Entwicklungsmethodik dar. In Kapitel 4 werden Modelle vorgestellt, die eine standardisierte Beschreibung und modellübergreifende Verwendung fertigungstechnologischen Wissens ermöglichen. Darauf aufbauend wird in Kapitel 5 eine umfassende Methodik vorgestellt, mittels derer fertigungsintegrierende Produktlösungen systematisch erarbeitet werden können. Kapitel 6 evaluiert diese Methodik anhand zweier Fallbeispiele. Kapitel 7 fasst die Kernaussagen der Arbeit noch einmal zusammen und gibt einen kurzen Ausblick über denkbare Erweiterungen der Methodik sowie weiterführende Forschungsfragen. Im Anhang finden sich ergänzende Unterlagen einschließlich eines Glossars. 


\section{Stand der Forschung}

Der Erfolg der Entwicklung fertigungsintegrierender Produktlösungen wird wesentlich durch das fertigungstechnologische Wissen beeinflusst, das dem Entwickler zur Verfügung steht. Bedingt durch die hohe Anzahl unterschiedlicher Fertigungstechnologien und die damit verknüpfte Menge an fertigungstechnologischem Wissen sind Ansätze notwendig, die den Produktentwickler bei der Verwendung des Wissens unterstützen. Das bedingt eine Auseinandersetzung mit dem in der Fertigungstechnik vorhandenen Wissen (siehe Abschnitt 2.1) und den vorhandenen Aufbereitungsformen dieses Wissens in der Produktentwicklung (siehe Abschnitt 2.2). Darauf aufbauend werden Ansätze im Rahmen des Design for Manufacture betrachtet, die fertigungstechnologisches Wissen für die Produktentwicklung nutzbar machen (siehe Abschnitt 2.3). Demgegenüber werden in Abschnitt 2.4 verschiedene Ansätze und Methodiken erläutert, die sich durch Verwendung einheitlicher Produkt- und Prozessmodelle zur Verknüpfung von Produkt- und Fertigungsprozesssicht deutlich von den DfM-Ansätzen abheben. Ergänzend wird aufgezeigt, wie Wissen ohne konkreten Themenbezug mittels verschiedener Wissensmanagementsysteme verarbeitet werden kann (siehe Abschnitt 2.5). Zwar finden sich auch in der Praxis unterschiedliche Herangehensweisen, um eine abteilungsübergreifende Produktentwicklung und Fertigung zu realisieren. Die folgenden Ausführungen sollen das Problem jedoch in erster Linie aus dem wissenschaftlichen Blickwinkel betrachten.

\subsection{Fertigungstechnologisches Wissen}

Der Begriff der Technologie beschreibt im allgemeinen Sprachgebrauch die wissenschaftliche Auseinandersetzung mit der „Umwandlung und Transformation von Roh- und Werkstoffen in fertige Produkte"19. Die Auseinandersetzung mit einer konkreten Fertigungstechnologie bedingt, Wissen über eine bestimmte Fertigungstechnik ${ }^{20}$ und damit über bestimmte Verfahren zu generieren und zu bewahren. In diesem Sinne beschreibt fertigungstechnologisches Wissen Sachverhalte, die sich je nach Fertigungstechnik bzw. Verfahren deutlich unterscheiden. Zudem findet sich dieses Wissen in unterschiedlichen Quellen wieder, die jeweils auf unterschiedliche Dokumentationsformen zurückgreifen. Fertigungstechnologisches

\footnotetext{
${ }^{19}$ Dudenredaktion (2017a).

20 Die Fertigungstechnik befasst sich damit, ein vorgegebenes Werkstück unter Verwendung geeigneter Fertigungsverfahren zu realisieren. (Vgl. Fritz, Schulze (2010), S. 1.)
} 
Wissens kann u. a. in Büchern, Datenbanken und Anleitungen abgelegt sein oder auch als mündliche Einweisung weitergereicht werden. Fertigungstechnologisches Wissen umfasst beispielsweise die mechanischen und werkstofflichen Eigenschaften gefertigter Bauteile. Wie in Abbildung 2-1 a) und b) dargestellt, werden häufig Graphen verwendet, um die mechanischen Eigenschaften der Bauteile zu charakterisieren. Abbildung 2-1 a) zeigt, wie sich die ertragbaren Spannungen durch Einsatz des Spaltprofilierens ändern sowie die Dehnungen, die bei unterschiedlichen Spannungen auftreten. So kann nachvollzogen werden, wie der Zusammenhang zwischen Spannungen und Dehnungen durch das Spaltprofilieren beeinflusst wird. Abbildung 2-1 b) verdeutlicht den Zusammenhang zwischen dem E-Modul im Bereich spaltprofilierter Flansche und der Richtung, in der die äußere Kraft aufgebracht wird.
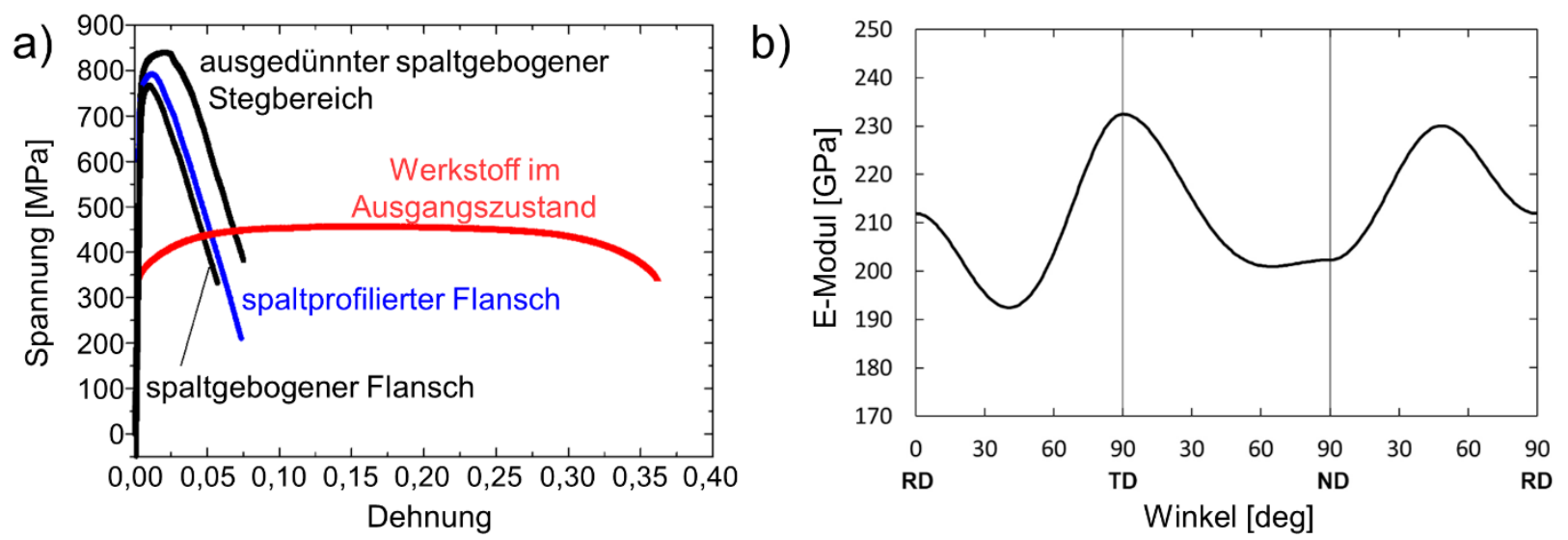

\begin{tabular}{|l|c|c|c|}
\hline \multirow{2}{*}{ Variationsparameter } & \multicolumn{2}{|c|}{ Auswirkungen bei Wertezunahme des Parameters } \\
\cline { 2 - 4 } & Walzenkräfte & $\begin{array}{c}\text { Versagensfreie } \\
\text { Gesamtspalttiefe }\end{array}$ & $\begin{array}{c}\text { Flanschrauheit } \boldsymbol{R}_{\mathbf{z}} \\
\text { (Oberseite) }\end{array}$ \\
\hline Spaltwalzenwinkel $\alpha$ & $\downarrow$ & 0 & $\downarrow$ \\
\hline Spaltwalzenradius $R$ & $\uparrow$ & $\uparrow$ & $\downarrow$ \\
\hline Spaltwalzendurchmesser $D_{\text {SW }}$ & $\uparrow$ & 0 & $\downarrow$ \\
\hline Inkrementelle Spalttiefe $y_{\text {ink }}$ & $\uparrow$ & $\uparrow$ & $\downarrow$ \\
\hline Flanschdicke $s_{\mathrm{f}}$ & $\downarrow$ & $\downarrow$ & $\uparrow$ \\
\hline
\end{tabular}

Abbildung 2-1: a) Spannungs-Dehnungskurven spaltprofilierter Bauteilproben im Vergleich zum Ausgangswerkstoff; ${ }^{21}$ b) Anisotropie des E-Moduls im Bereich spaltprofilierter Flansche ${ }^{22}$; c) Sensitivität von Prozessparametern und Eigenschaften beim Spaltprofilieren ${ }^{23}$

\footnotetext{
${ }^{21}$ Ahmels et al. (2017), S. 108.

22 Niehuesbernd et al. (2013).

${ }^{23}$ In Anlehnung an Jöckel (2005), S. 108.
} 
Demgegenüber zeigt Abbildung 2-1 c) eine tabellarische Übersicht über die Auswirkungen verschiedener Variationsparameter beim Spaltprofilieren auf Prozessparameter (Walzenkräfte und versagensfreie Gesamtspalttiefe) und Bauteileigenschaften (Flanschrauheit). Die Angabe der Zusammenhänge in Tabellenform gibt eine eindeutige Struktur für die dokumentierten Inhalte vor. Da es sich jedoch um qualitative Aussagen handelt, weisen sie inhaltlich einen deutlich geringeren Informationsgehalt auf als die zuvor dargestellten Graphen. Abbildung 2-2 zeigt hingegen einen Graphen, mittels dessen die beim Spaltprofilieren auftretenden Prozesskräfte am Werkzeugsystem deutlich präziser dokumentiert werden. Die Kräfte sind in Abhängigkeit der Stichtiefe $y_{\text {tot }}$ und des Spaltwalzenwinkels $\alpha$ aufgetragen. Das durch den Graphen dargestellte Spektrum möglicher Kombinationen der genannten Prozessparameter bildet die Grundlage zur Beherrschung des Fertigungsprozesses.
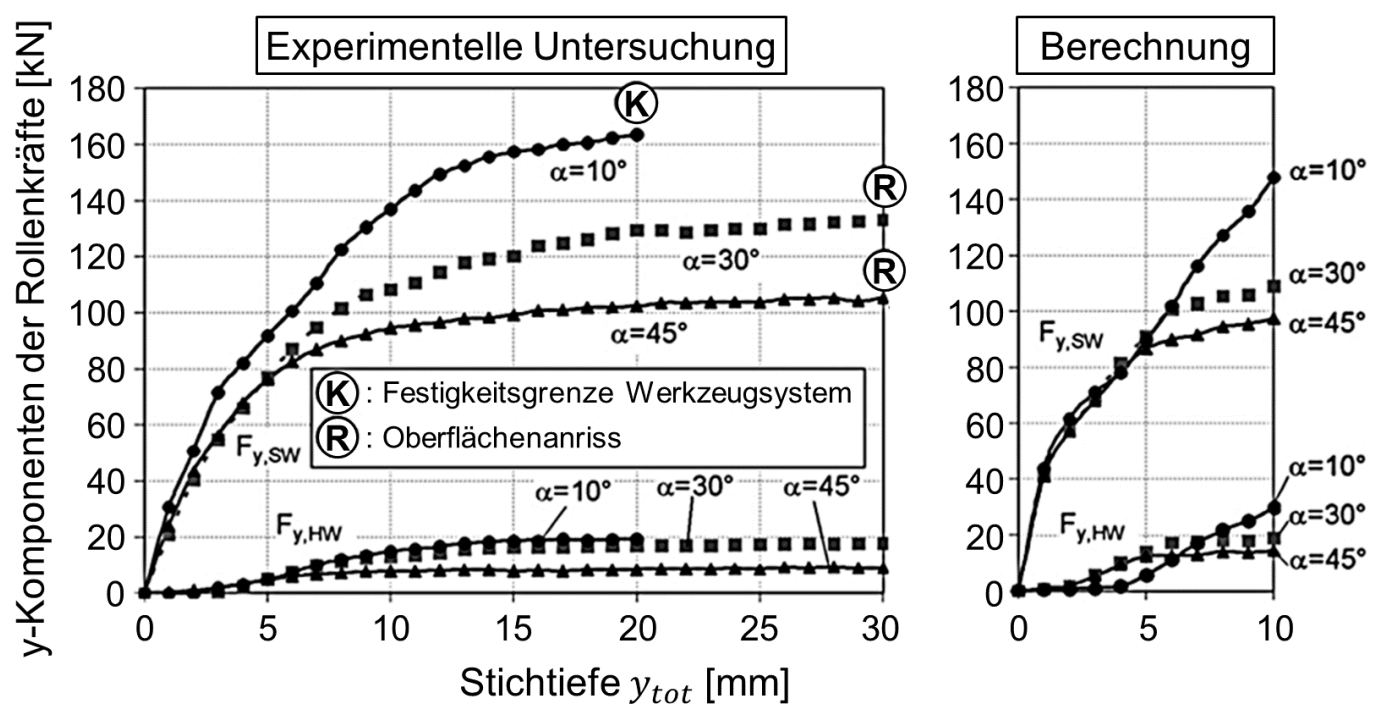

Abbildung 2-2: Experimentell und numerisch ermittelte Reaktionskräfte ${ }^{24}$

Fertigungstechnologisches Wissen findet sich häufig auch in textueller Form. Gerade bei einfachen Zusammenhängen, wie z. B. der Angabe erzeugbarer Bauteilformen und -abmessungen für ein gewähltes Fertigungsverfahren, bietet sich diese Dokumentationsform an. So findet sich die Aussage: „Bei Verwendung eines Stahls ZStE 340 können in zehn Umformstufen Flanschspannweiten von 22 mm, bei ZStE 500 bis zu $30 \mathrm{~mm}$ hergestellt werden. “25

${ }^{24}$ Groche et al. (2007), S. 254.

${ }^{25}$ Groche et al. (2012b), S. 87. 
Ebenso findet sich auch für andere Fertigungstechnologien fertigungstechnologisches Wissen, das das Zusammenwirken der am Prozess beteiligten Elemente mit Blick auf die wesentlichen Prozessparameter erklärt. Prinzipdarstellungen, wie am Beispiel des Innenhochdruckumformens in Abbildung 2-3 zu erkennen, können das Zusammenwirken der am Prozess beteiligten Elemente auf einfache Art und Weise erklären. Beim Innenhochdruckumformen werden zylindrische Rohre zu Hohlkörpern mit komplexeren Geometrien umgeformt. Dazu kommt ein Werkzeugsystem zum Einsatz, das die Negativform des späteren Bauteils abbildet. Oberund Unterwerkzeug werden durch eine Schließkraft $F_{\text {Schließ }}$ zusammengehalten. Zwei Stempel dichten das Rohr durch Aufbringen von Axialkräften $F_{\text {axial }}$ ab. Das mit einer Flüssigkeit gefüllte Rohr wird unter dem Innendruck $p_{\mathrm{i}}$ soweit umgeformt, bis es vollständig am Werkzeug anliegt. Die zulässige Obergrenze des zu Beginn der Umformung aufgebrachten Innendrucks $p_{\text {izul }}$ kann mittels der Kesselformel bestimmt werden. Der Druck wird darin in Abhängigkeit von der Geometrie und dem Werkstoff des verwendeten Halbzeugs beschrieben (siehe Abbildung 2-3).

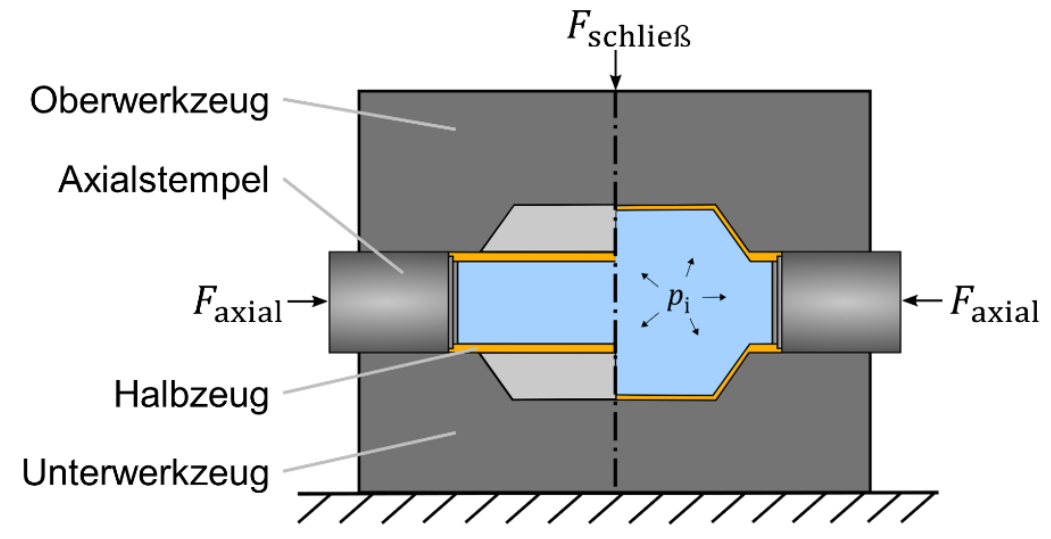

$p_{\mathrm{i} \mathrm{zul}} \approx \frac{2 \cdot s_{0} \cdot R_{\mathrm{m}}}{D_{0}-2 \cdot s_{0}}$

$p_{\text {i zul }}$ Zulässiger Innendruck

$s_{0} \quad$ Ausgangswanddicke

$D_{0} \quad$ Rohrausgangsdurchmesser

$R_{\mathrm{m}} \quad$ Zugfestigkeit

Abbildung 2-3: Innenhochdruckumformen unter Angabe des zulässigen Innendrucks ${ }^{26}$

Durch die Kesselformel wird nicht nur ersichtlich, wie die einzelnen Prozessparameter zusammenhängen. Durch gezieltes Anpassen der Parameter kann direkt Einfluss auf die erreichbaren Eigenschaften der gefertigten Bauteile genommen werden. Die mathematische Formel beschreibt damit einen Zusammenhang, der auf der Vernetzung fertigungstechnologischer Informationen beruht. Nur durch dieses Wissen ist es möglich, den Prozess innerhalb der durch Halbzeug, Werkzeugsystem und Prozesskräfte gegebenen Grenzen sinnvoll auszulegen. Zusätzliches Wissen über die Prozessparameter beim Innenhochdruckumformen und

${ }^{26}$ In Anlehnung an Noack, Sterzing (2007), S. 15 und VDI 3146-1 (1999). 
darüber, wie diese eingestellt werden müssen, findet sich in Abbildung 2-4. Sowohl eine tabellarische Übersicht möglicher thermomechanischer Prozesswege sowie eine Gegenüberstellung von Axialkraft und Innendruck geben nicht nur Aufschluss über den zeitlichen Ablauf des Prozesses, sondern auch über mögliche Prozessfenster. Insbesondere die Angabe der Prozessfenster zeigt deutlich, dass fertigungstechnologisches Wissen nicht immer den hohen Formalisierungsgrad einer mathematischen Formel aufweisen muss oder so konkrete Aussagen zulässt, wie dies mittels gemessener Spannungs-Dehnungskurven der Fall ist.

a)

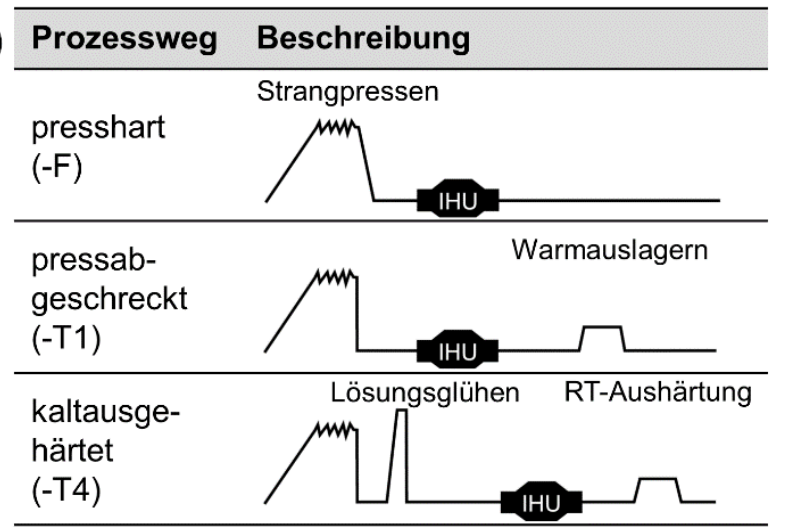

b)

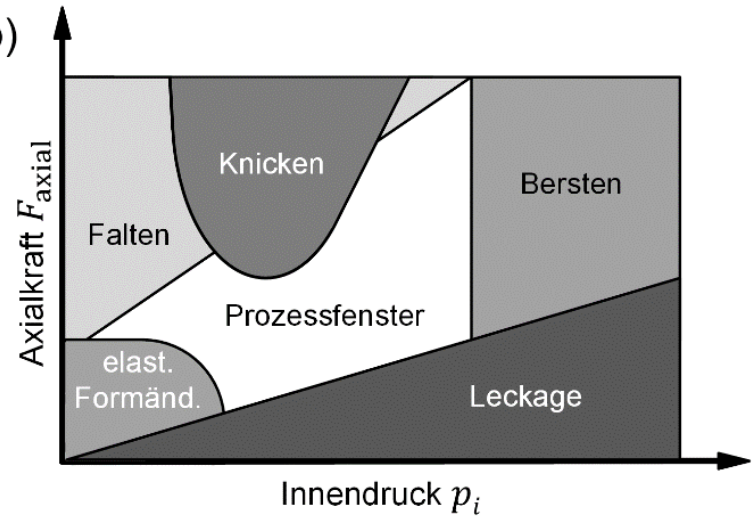

Abbildung 2-4: a) Thermomechanische Prozesswege ${ }^{27}$ und b) Prozessfenster beim Innenhochdruckumformen ${ }^{28}$

Die genannten Beispiele verdeutlichen, dass fertigungstechnologisches Wissen sowohl inhaltlich als auch formal sehr unterschiedlich dokumentiert sein kann. Zudem werden die dokumentierten Aussagen teils unterschiedlich konkret wiedergegeben. So werden Graphen, mathematische Formeln, Tabellen oder auch Abbildungen verwendet, um bestimmte Informationen zu Prozessparametern, Werkzeugen, Halbzeugen, etc. in Zusammenhang zu setzen. Die daraus resultierende Heterogenität fertigungstechnologischen Wissens erschwert dessen Verwendung insbesondere für die Entwicklung fertigungsintegrierender Produktlösungen.

\subsection{Dokumentationsformen für fertigungstechnologisches Wissen im Rahmen der Produktentwicklung}

Fertigungstechnologisches Wissen wird dem Produktentwickler meist in textgebundener und/oder bildlicher Form zur Verfügung gestellt. Das Wissen gibt Anhaltspunkte darüber, welche Verfahrensgrenzen in Form von Fertigungs- und

${ }^{27}$ Bahn et al. (2007), S. 51 in Anlehnung an Kunz (1998).

${ }^{28}$ Bahn et al. (2007), S. 80 in Anlehnung an VDI 3146-1 (1999). 
Montageprozessrestriktionen bei einer konkreten Problemstellung einzuhalten sind, aber auch wie eine vorteilhafte Bauteilgestalt aussehen kann. Die Verwendung textgebunden dokumentierter Hinweise, Regeln und (Gestaltungs-)Richtlinien gewährt einen einfachen und schnellen Zugriff auf das hinterlegte Wissen (siehe Tabelle 2-1). Gestaltvorbilder (siehe Tabelle 2-1) machen Wissen zu Fertigung und Montage in bildlicher Form zugänglich. Sie illustrieren anhand Beispielbauteilen, fiktiven Objekten oder Gut-Schlecht-Beispielen, wie ein Bauteil fertigungs- bzw. montagegerechter gestaltet werden kann. ${ }^{29}$

Tabelle 2-1: Gestaltungsrichtlinien und Beispiele zum fertigungsgerechten Gestalten von Blechteilen ${ }^{30}$

Anzustreben
$\begin{aligned} & \text { Vermeiden komplexer } \\ & \text { Biegeteile (Materialver- } \\ & \text { schnitt), dann besser } \\ & \text { teilen und fügen }\end{aligned}$
$\begin{aligned} & \text { Anstreben großer blei- } \\ & \text { bender Öffnungen bei } \\ & \text { Hohlkörpern und hinter- } \\ & \text { schnittenen Biegungen }\end{aligned}$

Einfache Schnitte, Verschnitt vermeiden
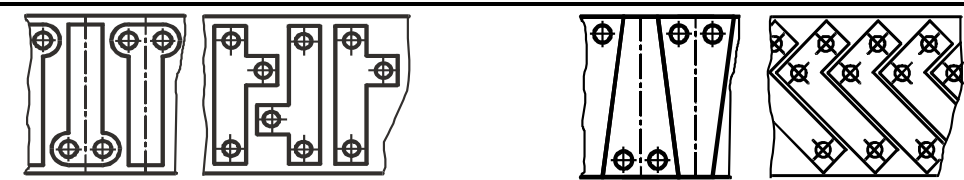

$\begin{aligned} & \text { Aussparungen mit Min- } \\ & \text { destabstand von Biege- } \\ & \text { kante }\end{aligned}$

Falls Mindestabstand nicht möglich, Aussparungen über Biegekante ziehen
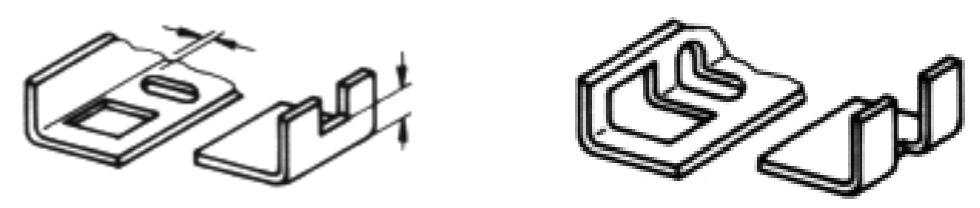

${ }^{29}$ Vgl. Andreasen et al. (1985); Ashby (2010); Bauer (2007); Boothroyd et al. (2010); Bralla (1999); Koller (1994); Meerkamm et al. (2012); Pahl et al. (2007). Grundlagen zur Konstruktion unter Einbeziehung des Einflusses bestimmter Fertigungstechnologien wurden bereits bei LEYER dokumentiert. (Vgl. Leyer (1963), S. 37 ff.)

${ }^{30}$ Gramlich (2013), S. 55 (Auszug aus Birkhofer, Nordmann (2002), S. 46 und Pahl et al. (2007), S. 459). 
Textgebundene Hinweise, Regeln und Richtlinien sowie bildliche Darstellungen können in Abhängigkeit der dokumentierten Inhalte grundsätzlich zu verschiedenen Entwicklungszeitpunkten angewendet werden. Durch deren kompakte Dokumentationsform wird das zugrunde liegende fertigungstechnologische Wissen jedoch nicht in Gänze wiedergegeben, sondern auf wesentliche Aussagen beschränkt, die beispielsweise eine günstige bzw. weniger günstige Gestaltung des Produkts hinsichtlich eines bestimmten Aspekts beschreiben. Zudem können auf der Grundlage des dokumentierten Wissens häufig keine Rückschlüsse über die Auswirkungen einer konstruktiven Festlegung des Produkts auf den Fertigungsprozess, z. B. die Prozessparameter betreffend, gegeben werden. So kann ein Entwickler aus der in Tabelle 2-1 dokumentierten Regel „Vermeiden komplexer Biegeteile (Materialverschnitt), dann besser teilen und fügen" Rückschlüsse für die Gestaltung seiner Blechbauteile ziehen. Ihm wird aber kein Einblick darin gewährt, welche konkreten Auswirkungen eine solche konstruktive Festlegung auf Montageprozesse hat. Ebenso kann er ohne weitere Angaben keine Aussage darüber treffen, ob die Kosten für die zusätzlich benötigten Werkzeugsysteme tatsächlich den geringeren Materialverschnitt rechtfertigen. Es ist vor allem die Erfahrung des Entwicklers notwendig, um das dokumentierte Wissen zu interpretieren und es auf das zu entwickelnde Produkt zu übertragen. Aufgrund des durch die gewählte Dokumentationsform beschränkten Zugriffs auf fertigungstechnologisches Wissen, gibt es zahllose weitere Möglichkeiten für die Entwicklung von fertigungsintegrierenden Produktlösungen, die bisher noch nicht ausgeschöpft werden, da sie nicht bekannt bzw. systematisch identifiziert wurden.

\subsection{Methoden und Werkzeuge zur Aufarbeitung und Nutzung von fertigungstechnologischem Wissen in der Produktentwicklung}

Jede konstruktive Festlegung, die während des Produktentwicklungsprozesses getroffen wird, hat nicht nur Auswirkungen auf die Produktgestalt, sondern auch auf entsprechende Lebenslaufprozesse (z. B. Fertigung, Montage, Recycling) sowie wesentliche Eigenschaften des Produkts (z. B. Kosten und Qualität). Dieses Bewusstsein begründet eine eigene Strömung innerhalb der Konstruktionswissenschaft, deren Ansätze unter dem Begriff „Design for X“ (DfX) zusammengefasst werden. Deren gemeinsames Ziel ist es, die Entwicklung und Konstruktion eines 
Produkts im Hinblick auf bestimmte Qualitätskriterien zu unterstützen. ${ }^{31}$ Unter das Themengebiet DfX fallen auch eine Reihe etablierter Ansätze, die sich mit der Berücksichtigung von Fertigung und Montage im Rahmen der Produktentwicklung beschäftigen. Sie werden als „Design for Manufacture“ (DfM) ${ }^{32}$ und „Design for Assembly" (DfA) bezeichnet. ${ }^{33}$ Dazu zählen auch spezialisierte Ansätze, die auf einzelne Fertigungstechnologien zugeschnitten sind, wie z. B. das „Design for Forming“. ${ }^{34}$ Im deutschsprachigen Raum werden diese Ansätze meist dem fertigungsgerechten Konstruieren bzw. dem montagegerechten Konstruieren zugeordnet. ${ }^{35}$ Aufgrund der starken Überschneidungen der Ansätze des DfM und DfA bietet sich auch eine gemeinsame Betrachtung im Rahmen des „Design for Manufacture and Assembly" (DfMA) an. ${ }^{36}$

Die Hauptzielsetzung des DfM liegt darin, dem Produktentwickler fertigungstechnologisches Wissen zur Unterstützung seiner konstruktiven Festlegungen zugänglich zu machen. ${ }^{37}$ Die Ansätze des DfM greifen dazu häufig auf die bereits genannten textgebundenen Hinweise, Regeln und Richtlinien sowie bildliche Darstellungen zurück. Gleiches gilt für die Ansätze des DfA, deren Fokus auf Wissen zu den Montageprozessen liegt. Auf diese Weise sollen ein geringerer Fertigungs- und Montageaufwand, geringere Durchlaufzeiten, geringere Kosten sowie eine geringere time-to-market bei gleichzeitiger Gewährleistung einer hohen Produktqualität erreicht werden. ${ }^{38}$ Existierende Ansätze zielen dazu auf eine Vereinfachung der Produkt- und Baustrukturen, eine stärker durch ökonomische Aspekte getriebene Auswahl von Materialien und Fertigungstechnologien sowie eine mit dem Ziel geringerer Fertigungskosten angepasste Feingestaltung ab (siehe Abbildung 2-5). ${ }^{39}$

Die dem Entwickler zur Verfügung stehenden DfM/DfA-Werkzeuge sind im Sinne einer möglichst zweckdienlichen Verwendung während der Produktentwicklung

\footnotetext{
${ }^{31} \mathrm{Vgl}$. Meerkamm et al. (2012), S. 446. ULRICH UND EPPINGER sprechen von Qualitätskriterien. (Vgl. Ulrich, Eppinger (2008), S. 221.) EHRLENSPIEL UND MEERKAMM verwenden den Begriff „nicht funktionsrelevante Forderungen“. (Ehrlenspiel, Meerkamm (2013), S. 354.)

32 Teilweise finden sich auch die Begriffe „Design for Manufacturing“ (vgl. Poli (2001)), „Design for Manufacturability“ (vgl. Anderson (2014)) und „Design for Production“ (vgl. Pahl et al. (2007), S. 445).

${ }^{33}$ Vgl. Meerkamm et al. (2012), S. 450; Herrmann et al. (2004), S. 2.

${ }^{34}$ Vgl. Altan, Miller (1990).

${ }^{35}$ Vgl. Pahl et al. (2007), S. 445 ff.; Meerkamm et al. (2012), S. 450 ff.; Andreasen et al. (1985).

${ }^{36} \mathrm{Vgl}$. Boothroyd et al. (2010).

${ }^{37} \mathrm{Vgl}$. Herrmann et al. (2004), S. 2.

${ }^{38}$ Vgl. Meerkamm et al. (2012), S. 451; Kesselring (1954); Pahl et al. (2007), S. 445.

${ }^{39}$ Vgl. Andreasen et al. (1985); Ashby (2010); Boothroyd et al. (2010); Bralla (1999); Pahl et al. (2007).
} 
meist so aufgebaut, dass sie die konstruktiven Festlegungen während bestimmter Phasen des Produktentwicklungsprozesses unterstützen. Während der frühen Entwicklungsphasen, in denen die prinzipielle Lösung für das zugrunde liegende Problem konzipiert wird, greifen Entwickler häufig auf Methoden wie das „Quality Function Deployment“ (QFD) zurück. Durch iteratives Einbeziehen von Fertigungsrestriktionen in die Überlegungen kann die Herstellbarkeit der Produkte frühzeitig abgesichert werden. Für die Erstellung des Entwurfs werden Softwaretools angeboten, die die Wahl von Werkstoff und Fertigungstechnologie unterstützen. Auch für die finale Ausarbeitung des Produkts existieren Werkzeuge, mittels derer eine bessere Herstellbarkeit der Produkte erreicht wird. Expertensysteme, „Failure Mode and Effects Analysis" (FMEA) und CAD-integrierte Analysesysteme auf Basis von Regeln stellen nur eine kleine Auswahl möglicher Werkzeuge dar. ${ }^{40}$

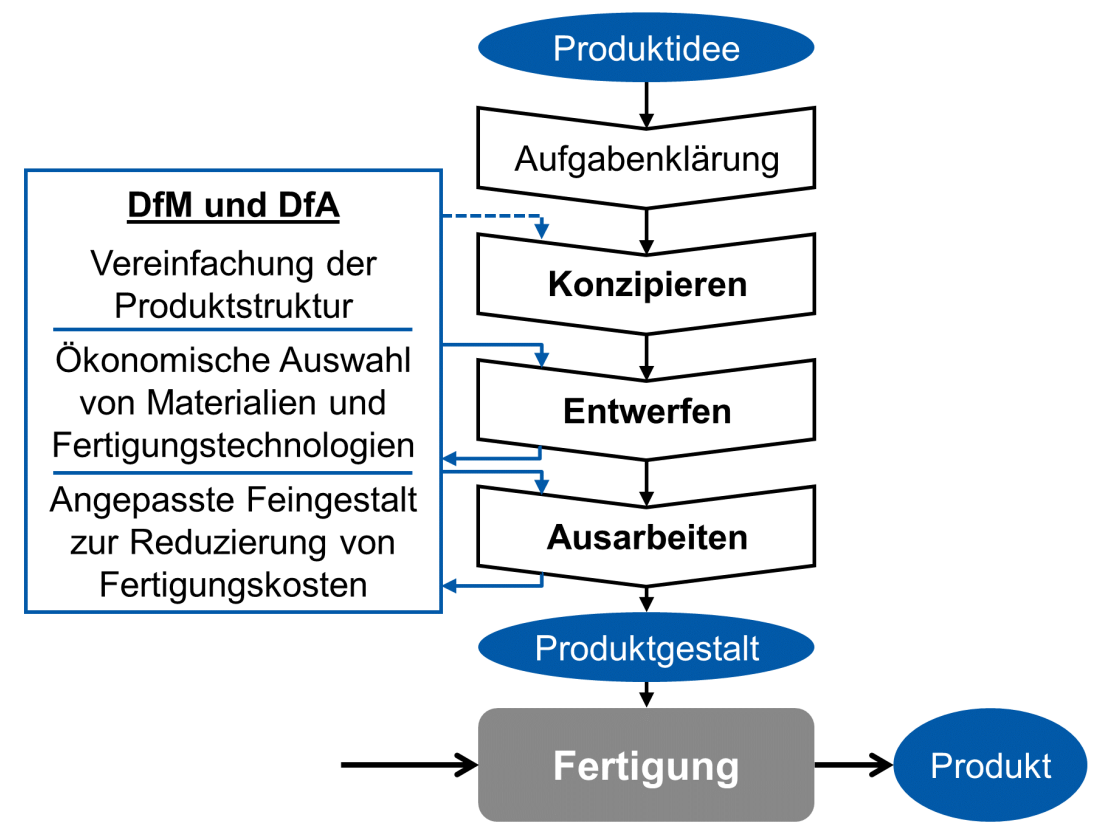

\section{Abbildung 2-5: Iterationsschleifen von DfM und DfA während des Produktentwicklungsprozesses nach VDI $2221^{41}$}

Auch wenn bereits Werkzeuge zur Unterstützung der frühen Entwicklungsphasen existieren, so führt die Anwendung von DfM/DfA-Ansätzen häufig zu zeitraubenden Iterationen in den späten Entwicklungsphasen (Entwerfen und Ausarbeiten) (siehe Abbildung 2-5). ${ }^{42}$ In den späten Entwicklungsphasen spielt zudem hauptsächlich das Sicherstellen der Herstellbarkeit des Produkts eine Rolle, was zur

\footnotetext{
${ }^{40}$ Vgl. Herrmann et al. (2004), S. 3.

41 Übersetzt nach Tekkaya et al. (2015), S. 648 in Anlehnung an Groche et al. (2012a), S. 164.

$42 \mathrm{Vgl}$. Groche et al. (2012a), S. 164.
} 
Folge hat, dass positive wie negative Einflüsse auf die Produktfunktion kaum berücksichtigt werden.

\subsection{Modellbasierte Methodiken zur Berücksichtigung der Fertigung im Kontext der Produktentwicklung}

Gegenüber den bisher vorgestellten Methodiken ${ }^{43}$, Methoden ${ }^{44}$ und Werkzeugen im Rahmen der Produktentwicklung, existieren solche, die Produkt- und Prozessmodelle nutzen, um die Möglichkeiten der Fertigung für die Produktentwicklung nutzbar zu machen. Der Einsatz von Modellen erlaubt es, die komplexe Realität vereinfacht und abstrahiert abzubilden. ${ }^{45}$

„Modelle sind vereinfachte abstrahierte Abbilder, Darstellungen oder gedankliche oder stoffliche Gebilde der komplexen Realität" ${ }^{46}$ mit einer dem Zweck der Modellbildung entsprechenden Genauigkeit. ${ }^{47}$

\subsubsection{Modellierung technischer Produkte}

Unter technischen Produkten werden Objekte verstanden, die der Lösung einer technischen Aufgabe dienen. ${ }^{48}$ Objekte sind stets geometrisch stoffliche Gebilde. $^{49}$

„Technische Produkte werden [...] als [...] technische Systeme oder Bestandteile übergeordneter Systeme verstanden und beschrieben." ${ }^{50}$

${ }^{43}$ Eine Methodik beschreibt ein planmäßiges Vorgehen, in das mehrere Methoden und Werkzeuge einbezogen werden. (Vgl. Lindemann (2009), S. 57; VDI 2223 (2004), S. 88.)

${ }^{44}$ Eine Methode beschreibt ein planmäßiges, regelbasiertes Vorgehen unter Angabe bestimmter Arbeitsschritte, mit deren Hilfe ein zuvor definiertes Ziel erreicht werden soll. Eine Methode gibt dazu Empfehlungen oder Anweisungen, die bei der Durchführung der Arbeitsschritte und der Dokumentation der Ergebnisse helfen sollen. (Vgl. Lindemann (2009), S. 56 f.; VDI 2223 (2004), S. 88.)

${ }^{45}$ Meist sind die Grenzen des menschlichen Denkvermögens ausschlaggebend für die Beherrschbarkeit. (Vgl. Heidemann (2001), S. 55; ebenso Birkhofer, Wäldele (2005).)

${ }^{46}$ Gramlich (2013), S. 11 in Anlehnung an Lindemann (2009), S. 331; VDI 2221 (1993), S. 41.

${ }^{47} \mathrm{Vgl}$. Pahl et al. (2007), S. 80.

${ }^{48} \mathrm{Vgl}$. Pahl et al. (2007), S. 39.

49 Vgl. Gramlich (2013), S. 62.

${ }^{50}$ Gramlich (2013), S. 14 in Anlehnung an Franke (1976), S. 186; Grote et al. (2008), S. K12. 


\section{Modelle technischer Produkte}

In Anlehnung an die Definition von Modellen können Produktmodelle als vereinfachte abstrahierte Abbilder des realen Produkts verstanden werden. Sie bilden alle produktdefinierenden Informationen ab, die während des Produktentwicklungsprozesses festgelegt werden. ${ }^{51}$ Im Rahmen der Konstruktionsmethodik werden in erster Linie Produktmodelle verwendet, deren Zweck darin liegt den Produktentwickler bei der methodischen Lösungsfindung zu unterstützen. ${ }^{52}$ Produktmodelle, die zur Unterstützung der Lösungsfindung eingesetzt werden, können in verschiedene Partialmodelle eingeteilt werden. EHRLENSPIEL ${ }^{53}$, ANDREASEN ${ }^{54}$ und SAUER ${ }^{55}$ definieren Partialmodelle, die sie verschiedenen Ebenen ihrer jeweiligen Produktmodellpyramide zuordnen. Sie eignen sich zur Systematisierung des Vorgehens während der Produktentwicklung. Die Komplexität und der Konkretisierungsgrad nehmen dabei in Richtung der unteren Ebenen zu. ${ }^{56}$

SAUER beschreibt eine Produktmodellpyramide, die sich in die Ebenen Funktionen, Effekte, Wirkprinzip und Gestalt gliedert (siehe Abbildung 2-6). Jeder dieser Ebenen wird ein Partialmodell der Produktentwicklung zugeordnet, um die den Ebenen zugeordneten Entwicklungsschritte gezielt zu unterstützen. Darüber hinaus findet sich der technische Prozess an der Spitze der Pyramide. Diese Anordnung basiert auf der Überlegung, dass der Zweck des Produkts durch den Einsatzprozess und dessen verfahrenstechnische Umsetzung festgelegt wird. ${ }^{57}$ Die Produktfunktion wird auf der obersten Ebene der Pyramide angeordnet. Die Zweckerfüllung eines technischen Produkts, repräsentiert durch dessen Funktion, wird jedoch erst durch deren technische Umsetzung erreicht. ${ }^{58}$ Dementsprechend dienen die weiteren Ebenen der Produktmodellpyramide der Unterstützung dieses Vorgehens.

\footnotetext{
${ }^{51}$ Vgl. Wäldele (2012), S. 28.

52 Vgl. Gramlich (2013), S. 24. Dieses Verständnis von Produktmodellen geht auf deren konstruktionsmethodische Verwendung zurück, wie sie bei Birkhofer (2011); Ehrlenspiel (2009); Andreasen, Hein (1987); Sauer (2006) verwendet wird. In Rahmen der Produktdatentechnologie werden ebenfalls Produktmodelle eingesetzt, deren Schwerpunkt auf der digitalen Repräsentation des Produkts liegt. So wird z. B. in der Norm ISO 10303 mit dem Standard for the Exchange of Product model data (STEP) ein Produktmodell vorgestellt, das die digitale Abbildung aller Produktdaten über sämtliche Produktlebenslaufphasen hinweg und deren Integration in ein einheitliches Produktdatenmodell ermöglicht. (Vgl. Anderl, Trippner (2000), S. 9 f.; Grabowski et al. (1993); Rollmann (2012), S. 17 ff.; ISO 10303-1 (1994).)

${ }^{53} \mathrm{Vgl}$. Ehrlenspiel (2009), S. $37 \mathrm{f}$.

${ }^{54} \mathrm{Vgl}$. Andreasen, Hein (1987).

${ }^{55}$ Vgl. Sauer (2006), S. $67 \mathrm{ff}$.

${ }^{56}$ Vgl. Wäldele (2012), S. 28.

57 Vgl. Sauer (2006), S. $67 \mathrm{ff}$.

${ }^{58} \mathrm{Vgl}$. Feldhusen (2008), S. 168.
} 


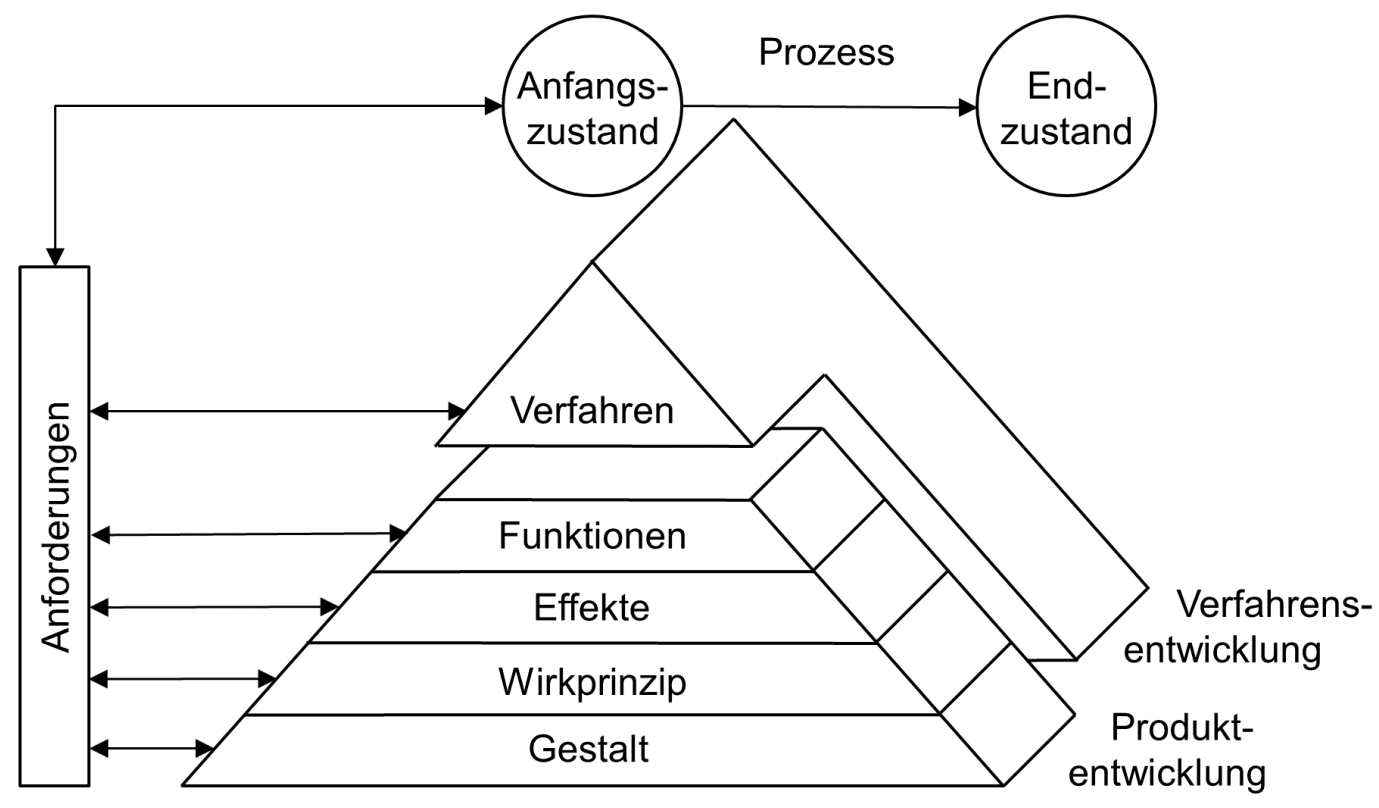

Abbildung 2-6: Produktmodellpyramide nach SAUER ${ }^{59}$

Aufbauend auf den Definitionen von PAHL ${ }^{60}$ und der VDI $2221^{61}$ gibt GRAMLICH eine Begriffsdefinition für die Funktion eines Produkts:

„Die Produktfunktion ist eine lösungsneutrale Beschreibung des kausalen Zusammenhangs zwischen gewollten Ein- und Ausgangsgrößen ${ }^{62}$ des technischen Produkts. Als Ein- und Ausgangsgrößen [...] [fungieren] ausschließlich Energien oder Signale“. 63

Dieser Definition liegt der Gedanke zugrunde, die Rolle von Objekten und Größen in den unterschiedlichen Modellen der Konstruktionswissenschaft zu differenzieren. Auf der einen Seite werden technische Objekte bzw. Produkte im Kontext ihrer Funktion modelliert. Auf der anderen Seite werden technische Objekte bzw. Produkte in technischen Prozessen verwendet. Größen beschreiben diese Objekte und Vorgänge im Kontext der technischen Prozesse. Sie sind selbst jedoch keine Objekte und somit nicht gegenständlicher Natur. Größen werden darüber hinaus im Kontext der Thermodynamik eindeutig in Zustandsgrößen und Prozessgrößen

\footnotetext{
59 Sauer (2006), S. 68.

60 Vgl. Pahl et al. (2007), S. 783.

${ }^{61}$ Vgl. VDI 2221 (1993), S. 40.

62 Im weiteren Verlauf der Arbeit wird der Begriff der Funktionsgrößen in Anlehnung an ROTH (vgl. Roth (2000), S. 113) verwendet.

${ }^{63}$ Vgl. Gramlich (2013), S. 164.
} 
unterteilt. ${ }^{64}$ Während Zustandsgrößen die Objekte in einem bestimmten Zustand des Prozesses beschreiben ${ }^{65}$, umfassen Prozessgrößen sowohl Energien als auch Signale die zwischen den Objekten ausgetauscht werden ${ }^{66}$. Während Energien u. a. mechanischer, thermischer, elektrischer, chemischer oder optischer $\mathrm{Na}$ tur sein können, fungieren Signale als Träger von Informationen ${ }^{67}$. Größen - im Sinne von Prozessgrößen - beschreiben somit keinen Zustand eines Objekts, sondern Energien und Signale die zwischen den Objekten ausgetauscht werden. ${ }^{68}$

Die für das Produkt definierte Gesamtfunktion kann zudem in Teilfunktionen (TF) unterteilt werden, die zueinander in Beziehung stehen. Auf diese Weise wird die Modellbildung in besser beherrschbare Teilprobleme zerlegt. ${ }^{69}$ Funktionen bzw. Teilfunktionen werden mithilfe physikalischer, biologischer oder chemischer Effekte realisiert, die auf physikalischen bzw. biologischen oder chemischen Gesetzmäßigkeiten (z. B. Hebelgesetz, Coulombsches Reibungsgesetz) beruhen. ${ }^{70}$ Diese Gesetzmäßigkeiten verknüpfen die Ein- und Ausgangsgrößen der Produktfunktion. ${ }^{71}$ Durch das Festlegen erster geometrischer und werkstofflicher Eigenschaften des Produkts im Partialmodell des Wirkprinzips werden Wirkelemente in einen Wirkzusammenhang gebracht. Das Wirkprinzip stellt somit ein wesentliches Bindeglied zwischen den physikalischen Effekten und den physischen Elementen des Produkts dar. ${ }^{72} \mathrm{Im}$ Gestaltmodell werden die Wirkelemente durch Gestaltelemente geometrisch und werkstofflich umgesetzt und damit die Produktgestalt vollständig festgelegt. ${ }^{73}$

\footnotetext{
${ }^{64} \mathrm{Vgl}$. Weigand et al. (2010), S. $5 \mathrm{ff} ., 174 \mathrm{f}$.

${ }^{65} \mathrm{Vgl}$. Baehr (2005), S. 13.

${ }^{66}$ Vgl. Gramlich (2013), S. 62 ff.

${ }^{67}$ Vgl. Tröster (2005), S. 37.

${ }^{68}$ Vgl. Gramlich (2013), S. 62 ff. Größen werden im Rahmen dieser Arbeit ausschließlich im Sinne von Prozessgrößen verwendet, da aufgrund der Beschreibung von Objekten mittels derer Eigenschaften die Verwendung von Zustandsgrößen eine inhaltliche Dopplung darstellen würde.

${ }^{69}$ Vgl. Pahl et al. (2007), S. $41 \mathrm{ff}$.

${ }^{70}$ Vgl. Koller (1994), S. 52; Wäldele (2012), S. 31 f. Häufig wird nur der Begriff „physikalischer Effekt" verwendet.

71 Vgl. Gramlich (2013), S. 82.

72 Vgl. Pahl et al. (2007), S. 52; Ponn, Lindemann (2008), S. 79.

${ }^{73}$ Vgl. Gramlich (2013), S. 73, 83; Wäldele (2012), S. 33.
} 


\section{Beschreibung technischer Produkte durch Eigenschaften}

Eine Eigenschaft wird als gedanklich abgegrenzter Teil der Beschreibung eines Objektes verstanden. ${ }^{74}$ Die Beschreibung mittels Eigenschaften ermöglicht die eindeutige Charakterisierung des Objekts sowie die präzise Differenzierung unterschiedlicher Objekte. ${ }^{75}$ Eigenschaften können durch Beobachtungen, Messungen, Berechnungen, etc. festgestellt werden. ${ }^{76}$ Sie sind qualitativer, quantitativer oder komparativer Natur und müssen nicht zwangsweise eine Einheit besitzen. ${ }^{77}$ BIRKHOFER definiert Eigenschaften in Anlehnung an LEINEMANN als eine Kombination aus einem Merkmal und einer Ausprägung aus einer merkmalspezifischen Menge möglicher Ausprägungen. ${ }^{78}$ So bildet beispielsweise die Kombination aus dem Merkmal "Steifigkeit“ und der Ausprägung „10 N/m" eine Eigenschaft, die der Beschreibung einer Schraubenfeder dient.

Eine Eigenschaft beschreibt ein Objekt in Form eines Merkmals und einer zugehörigen Ausprägung, wobei die Ausprägung einer merkmalspezifischen Menge möglicher Ausprägungen entstammt. ${ }^{79}$

Eigenschaften, die der Beschreibung technischer Produkte dienen, werden als Produkteigenschaften bezeichnet. ${ }^{80}$ BIRKHOFER UND WÄLDELE unterteilen Produkteigenschaften in unabhängige und abhängige Produkteigenschaften. Die unabhängigen Produkteigenschaften können unabhängig von anderen Eigenschaften festgelegt werden. Durch die Summe dieser Produkteigenschaften ist das Produkt vollständig festgelegt. Die abhängigen Produkteigenschaften können demgegenüber stets nur indirekt durch Festlegen der unabhängigen Produkteigenschaften festgelegt werden. ${ }^{81}$ Abbildung 2-7 zeigt eine Schraubenfeder unter Angabe der Merkmale unabhängiger und abhängiger Produkteigenschaften. Steifigkeit,

\footnotetext{
${ }^{74}$ Vgl. Gramlich (2013), S. 17.

${ }^{75} \mathrm{Vgl}$. Birkhofer (1980), S. 5. BIRKHOFER bezieht diese Aussage auf technische Systeme.

${ }^{76}$ Vgl. Wäldele (2012), S. 16.

77 Vgl. Gramlich (2013), S. 18.

${ }^{78}$ Vgl. Leinemann (1974), S. 439; Birkhofer (1980), S. 6. In aktuelleren Veröffentlichungen wird statt des Begriffs Wert der Begriff Ausprägung verwendet. (Vgl. Ehrlenspiel (2009), S. 28; Lindemann (2009), S. 160.)

${ }^{79}$ Vgl. Birkhofer (1980), S. 5 f.; Gramlich (2013), S. 17 f.

${ }^{80}$ Bei den meisten Autoren werden die Begriffe Eigenschaft und Produkteigenschaft synonym verwendet. Der Begriff Produkteigenschaft wird u. A. bei WÄLDELE und GRAMLICH verwendet. (Vgl. Wäldele (2012); Gramlich (2013).)

${ }^{81}$ Vgl. Birkhofer, Wäldele (2008), S. 4 f.; Wäldele (2012), S. 56 ff.
} 
Dämpfung, Masse und Volumen der Schraubenfeder hängen u. a. von Federlänge, Federdurchmesser, Drahtdurchmesser, Werkstoff und Windungszahl ab.

\begin{tabular}{l}
\hline Unabhängige \\
Produkteigenschaften \\
\hline - Federlänge \\
- Federdurchmesser \\
- Drahtdurchmesser \\
- Werkstoff \\
- Windungszahl
\end{tabular}

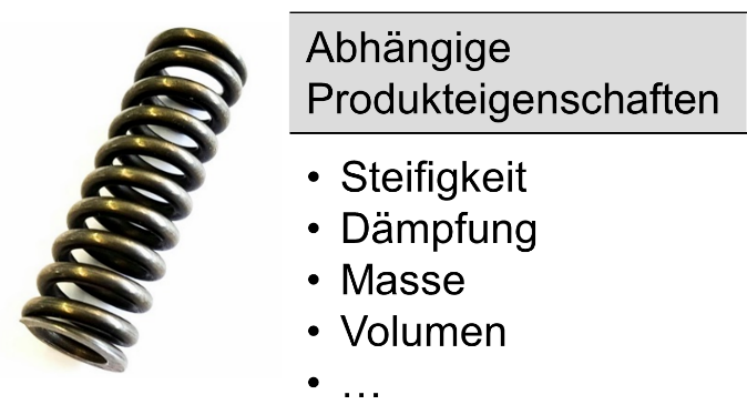

Abbildung 2-7: Unabhängige und abhängige Produkteigenschaften einer Schraubenfeder

An dieser Stelle sei noch die Kategorisierung von Eigenschaften nach WEBER erwähnt. Im Rahmen seines Characteristics-Properties Modelling (CPM) unterscheidet er zwischen Merkmalen (Characteristics) und Eigenschaften (Properties) eines technischen Systems. ${ }^{82}$ Diese Kategorisierung ist der Unterscheidung in unabhängige und abhängige Eigenschaften inhaltlich sehr ähnlich.

\section{Eigenschaften in Produktmodellen}

Zur Beschreibung des technischen Produkts existieren auf jeder Ebene der Produktmodellpyramide modellspezifische Produkteigenschaften, die als Produktmodelleigenschaften bezeichnet werden. Diese lassen sich gemäß der oben genannten Unterscheidung in unabhängige und abhängige Produktmodelleigenschaften unterteilen. ${ }^{83}$ Auf der Funktionsebene werden auch die Ein- und Ausgangsfunktionsgrößen mit in die Modellierung einbezogen. ${ }^{84}$ Die Produktgestalt wird durch die Summe der unabhängigen Gestalteigenschaften beschrieben. Diese umfassen neben geometrischen Eigenschaften auch werkstoffliche Eigenschaften ${ }^{85}{ }^{86}$ Werkstoffliche Eigenschaften bzw. Eigenschaften, die vom verwendeten Material dominiert werden, umfassen zudem mechanische Eigenschaften (Festigkeit, Härte,

\footnotetext{
${ }^{82} \mathrm{Vgl}$. Weber (2012), S. 31; Weber (2005), S. 163.

${ }^{83}$ Vgl. Gramlich (2013), S. $79 \mathrm{ff}$.

${ }^{84}$ Vgl. Gramlich (2013), S. 80.

${ }^{85}$ In der Literatur finden sich auch die Begriffe stoffliche Eigenschaften und Materialeigenschaften. (Vgl. Gramlich (2013), S. 73; Birkhofer, Wäldele (2008), S. 22.)

${ }^{86}$ Vgl. Gramlich (2013), S. 83, 162; Ponn, Lindemann (2008), S. 124.
} 
Dauerfestigkeit, Umformbarkeit, Eigenspannungen, etc.), Eigenschaften der Oberfläche ${ }^{87}$ (Oberflächentopologie, Reibwiderstand, Korrosionsbeständigkeit, etc.) und physikalische Eigenschaften (elektrische Eigenschaften, magnetische Eigenschaften, optische Eigenschaften, etc.). Ergänzend können auch Eigenschaften über den energetischen, informationstechnischen sowie orts- und lagebezogenen Zustand erfasst werden. ${ }^{88}$

Die Modellierung einer Schraubenfeder bedingt die Beschreibung durch verschiedene Produktmodelleigenschaften auf verschiedenen Ebenen der Produktmodellpyramide (siehe Tabelle 2-2). Die Beschreibung ist durch Angabe der Merkmale bis auf die Gestaltebene konkretisiert. Die Produktfunktion der Schraubenfeder wird durch ihre Eingangsfunktionsgröße $\Delta F$ und ihre Ausgangsfunktionsgröße $\Delta s$ beschrieben (siehe Abbildung 2-8 b). Mit zunehmendem Konkretisierungsgrad kommen weitere Produkteigenschaften dazu.

Tabelle 2-2: Produktmodelleigenschafen einer Schraubenfeder

\begin{tabular}{|c|c|c|c|}
\hline Modellebene & Produktmodell & eigenschaften & \\
\hline Produktfunktion & & $\Delta F=f(\Delta s)$ & $\begin{array}{l}\Delta F: \text { Kraft } \\
\Delta s: \text { Auslenkung }\end{array}$ \\
\hline $\begin{array}{l}\text { Physikalischer } \\
\text { Effekt }\end{array}$ & $\begin{array}{l}\text { elastische } \\
\text { Verformung }\end{array}$ & $\Delta F=c \cdot \Delta s$ & $c$ : Steifigkeit \\
\hline Wirkprinzip & Torsionsstab & $\begin{array}{l}\Delta M_{\mathrm{t}}=\frac{G \cdot I_{\mathrm{p}}}{L} \Delta \varphi \\
\text { mit } M_{\mathrm{t}}=r \cdot F \\
\text { und } \Delta \varphi=\frac{\Delta s}{r} \\
\text { für kleine Winkel }\end{array}$ & $\begin{array}{l}M_{\mathrm{t}}: \text { Torsionsmoment } \\
G: \text { Schubmodul } \\
I_{\mathrm{p}}: \text { polares Flächen- } \\
\text { trägheitsmoment } \\
L: \text { Stablänge } \\
\Delta \varphi: \text { Verdrehwinkel } \\
r: \text { radialer Abstand } \\
\text { zum Kraftangriffspunkt }\end{array}$ \\
\hline Produktgestalt & Schraubenfeder & $\begin{array}{l}\Delta F=\frac{G \cdot d_{\mathrm{D}}^{4}}{\left(8 \cdot d_{\mathrm{F}}^{3} \cdot n\right)} \Delta s \\
\text { mit } d_{\mathrm{F}}=2 \cdot r\end{array}$ & $\begin{array}{l}d_{\mathrm{D}}: \text { Drahtdurchmesser } \\
d_{\mathrm{F}}: \text { Federdurchmesser } \\
n: \text { Anzahl Windungen }\end{array}$ \\
\hline
\end{tabular}

${ }^{87}$ Eigenschaften der Oberfläche umfassen korrekterweise sowohl werkstoffliche Eigenschaften, wie Reibwerte oder Korrosionsbeständigkeit als auch geometrische Eigenschaften, wie die Oberflächentopologie. Um dennoch eine Eindeutigkeit in der Zuordnung zu ermöglichen, werden Eigenschaften der Oberfläche vereinfacht den werkstofflichen Eigenschaften zugeordnet.

${ }^{88}$ Vgl. Gramlich (2013), S. 73; Wäldele (2012), S. 33; Tekkaya et al. (2015), S. 630. 
Die Funktion wird durch den physikalischen Effekt der elastischen Verformung konkretisiert. Die Steifigkeit $c$ fungiert auf dieser Ebene als unabhängige Produktmodelleigenschaft, die das Zusammenspiel der Funktionsgrößen beschreibt. Für eine Schraubenfeder konkretisiert sich die elastische Verformung durch einen Torsionsstab (siehe Abbildung 2-8 a).
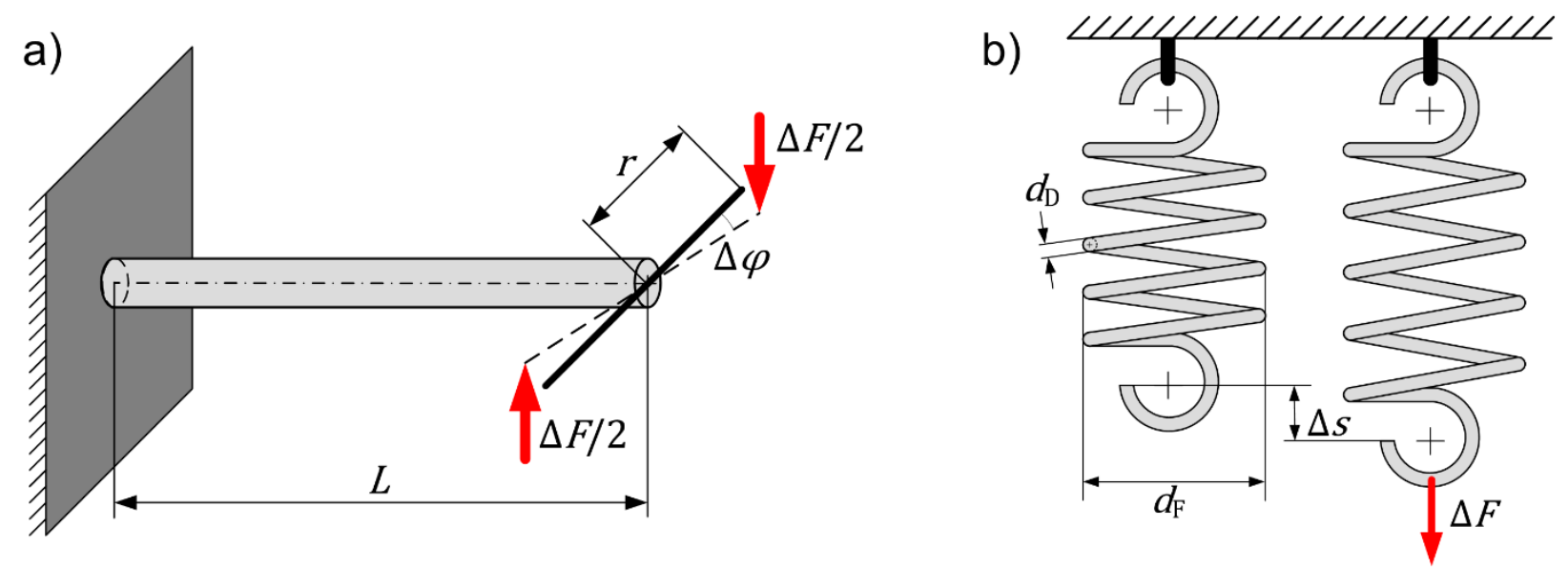

Abbildung 2-8: a) Torsionsstab; b) Schraubenfeder unter Zugbelastung

Die Steifigkeit des Torsionsstabs ist auf der Modellebene des Wirkprinzips nur noch eine abhängige Produktmodelleigenschaft, die von den unabhängigen Produktmodelleigenschaften Schubmodul $G$, polares Flächenträgheitsmoment $I_{\mathrm{p}}$ und Stablänge $L$ abhängt. Zusätzliche geometrische Zusammenhänge ermöglichen die Verknüpfung der ursprünglichen Funktionsgrößen mit dem Torsionsmoment $\Delta M_{\mathrm{t}}$ und dem Verdrehwinkel $\Delta \varphi$. Die Produktgestalt wird durch die unabhängigen Gestalteigenschaften in Bezug auf Werkstoff (Schubmodul $G$ ) und Geometrie (Drahtdurchmesser $d_{\mathrm{D}}$, Federdurchmesser $d_{\mathrm{F}}$, und Anzahl der Windungen $n$ ) beschrieben.

Die den Produktmodellen zugrunde liegenden Zusammenhänge können in Form von Produktmodelleigenschaften oder Größen und deren Relationen miteinander verknüpft werden. ${ }^{89}$ "Größen beschreiben [...] Eigenschaften von Objekten und Vorgängen und setzen sich daher, genau wie Eigenschaften, aus einem Merkmal und einer Ausprägung zusammen. " ${ }^{90}$ Aufbauend auf der oben aufgeführten Erläuterung kann die folgende Definition zu Größen gegeben werden.

\footnotetext{
${ }^{89}$ Vgl. Gramlich (2013), S. 76 ff.; Wäldele (2012), S. 70 ff. Eigenschaftsrelationen bilden demnach die Abhängigkeiten und Beziehungen zwischen Eigenschaften ab.

${ }^{90}$ Gramlich (2013), S. 63.
} 
Größen - im Sinne von Prozessgrößen - beschreiben keinen Zustand eines Objekts, sondern Energien und Signale die zwischen den Objekten ausgetauscht werden. Sie setzen sich aus einem Merkmal und einer zugehörigen Ausprägung zusammen. ${ }^{91}$

Eigenschaftsrelationen können in netzwerkartigen Strukturen abgebildet werden. ${ }^{92}$ Eigenschaftsnetzwerke sind jedoch in Bezug auf die Aspekte Übersichtlichkeit und Nachverfolgbarkeit in der Anzahl darstellbarer Relationen limitiert. Abbildung 2-9 zeigt den Zusammenhang zwischen der Steifigkeit $c$ einer Schraubenfeder und deren unabhängigen Gestalteigenschaften in Form eines Eigenschaftsnetzwerks. Das Produktmodell der Schraubenfeder wird durch ein mechanisches Ersatzmodell und eine entsprechende Formel für die Federsteifigkeit $c$ abgebildet. Es gibt den Zusammenhang zwischen der Absenkung $\Delta s$ und der Kraft $F$ in Abhängigkeit von der Federsteifigkeit $c$ an.
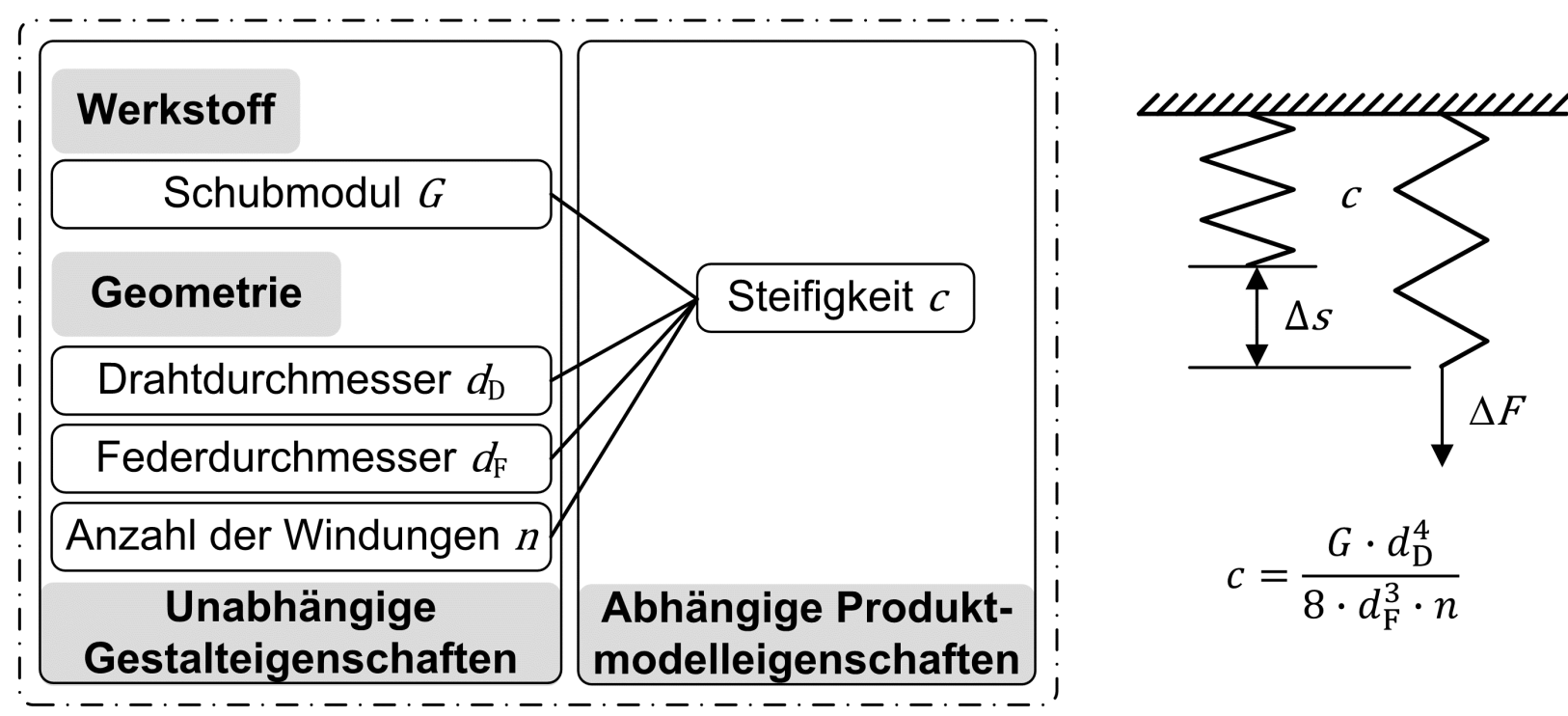

$$
c=\frac{G \cdot d_{\mathrm{D}}^{4}}{8 \cdot d_{\mathrm{F}}^{3} \cdot n}
$$

Abbildung 2-9: Mittels Eigenschaften beschriebenes Modell einer Schraubenfeder ${ }^{93}$

\footnotetext{
${ }^{91}$ Vgl. Gramlich (2013), S. $62 \mathrm{ff.}$

92 Vgl. Wäldele (2012), S. $81 \mathrm{ff}$.

${ }^{93}$ Aus dem Englischen übersetzt von Lüthen et al. (2017), S. 152.
} 


\section{Strukturierung technischer Produkte}

Mit steigender Komplexität technischer Produkte wird auch deren Modellierung immer schwieriger. Im Einklang mit dem hierarchischen Systemkonzept nach RoP$\mathrm{OHL}^{94}$ ist es sinnvoll, technische Produkte in beherrschbare Elemente zu strukturieren. Abhängig vom zugrunde gelegten Modell werden die Produkte unter Zuhilfenahme von Eigenschaften und zugehöriger Relationen in Elemente (vgl. auch Wirkelemente, Gestaltelemente ${ }^{95}$ ) zerlegt. In Abbildung 2-10 a) ist beispielsweise eine Profilstruktur dargestellt, die sich aus Profilelementen (Träger und Knoten) zusammensetzt. Die Profilelemente werden mittels Eigenschaften beschrieben. Die einzelnen Elemente stehen mittels Eigenschaftsrelationen wiederum innerhalb eines übergeordneten Eigenschaftsnetzwerks in Beziehung. ${ }^{96}$

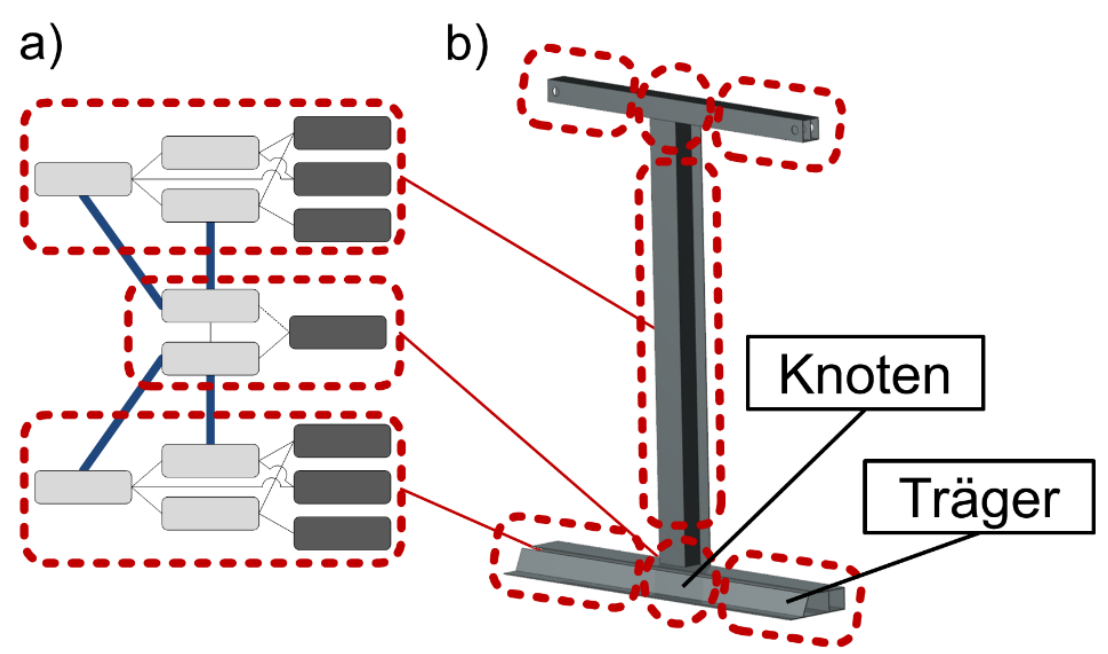

Abbildung 2-10: a) Eigenschaftsnetzwerk und b) Funktionsträger eines Posterständers ${ }^{97}$

Eine diesem Gedanken entsprechende Möglichkeit, das technische Produkt zu strukturieren, setzt bei der Produktfunktion an. Ausgehend von der Strukturierung der Gesamtfunktion in Teilfunktionen, können bestimmte Elemente des Produkts identifiziert werden, die gemeinsam zur Erfüllung der jeweiligen Teilfunktion beitragen. Diese Elemente werden als Funktionsträger bezeichnet. Dabei muss es sich nicht zwangsweise um physisch separierte Bauteile des technischen Produkts

\footnotetext{
${ }^{94} \mathrm{Vgl}$. Ropohl (2009), S. 75 f.

${ }^{95}$ Gestaltelemente sind im Rahmen dieser Arbeit die Elemente, in die sich die Produktgestalt zerlegen und damit strukturieren lässt. Sie werden mittels Gestalteigenschaften geometrisch und werkstofflich beschrieben. (Vgl. Gramlich (2013), S. 80.)

${ }^{96}$ Vgl. Gramlich et al. (2011), S. 302 f.

${ }^{97}$ In Anlehnung an Gramlich et al. (2011), S. 303.
} 
handeln. Funktionsträger können sowohl die komplette Funktion als auch die durch physikalische Effekte oder Wirkprinzipien konkretisierte Funktion bzw. Teile davon repräsentieren. ${ }^{98}$ Funktionsträger werden durch unabhängige und abhängige Eigenschaften beschrieben, die zueinander in Relation stehen.

Funktionsträger sind konstruktionsbestimmende Elemente, die die Funktion des Produkts bestimmen. Funktionsträger können Teile, Lösungsprinzipien oder Prinziplösungen sein, die eine oder mehrere Funktionen erfüllen. ${ }^{99}$

Der in Abbildung 2-10 dargestellte Posterständer kann so z. B. in Träger und Knoten aufgeteilt werden. Die Knoten verteilen alle auftretenden Kräfte an die angeschlossenen Träger (Funktion: Kraft verteilen), die wiederum die Kräfte zwischen den Knoten und Kraftangriffspunkten leiten (Funktion: Kraft leiten). Anhand der gewählten Konstruktion wird ersichtlich, dass die Strukturierung der Funktionsträger nicht zwangsweise mit der Bauteilstruktur, bestehend aus drei Profilelementen, korrelieren muss.

\subsubsection{Modellierung technischer Prozesse}

Im Kontext der Produktentwicklung werden technische Prozesse als Transformationsprozesse aufgefasst. Sie beschreiben die definierte Zustandsänderung eines Operanden. ${ }^{100}$ Technische Produkte sind das (Arbeits-)Mittel, um im Rahmen technischer Prozesse den Zweck der gewollten Zustandsänderung eines oder mehrerer Objekte zu erfüllen (Zweck-Mittel-Differenzierung). ${ }^{101}$ Mit der bereits genannten strikten Differenzierung von Objekten und (Prozess-) Größen schränkt GRAMLICH technische Prozesse auf die Zustandsänderung stofflicher Gebilde ein. Energien und Informationen können somit nicht als Operanden auftreten, es sei denn sie sind an stoffliche Gebilde, also Objekte, gebunden. ${ }^{102}$

\footnotetext{
${ }^{98}$ Vgl. Pahl et al. (2007), S. 256, 308, 783; Ehrlenspiel, Meerkamm (2013), S. 46 f., 745; Wagner et al. (2016), S. 742.

99 Vgl. Pahl et al. (2007), S. 256, 308, 783; Ehrlenspiel, Meerkamm (2013), S. 46 f., 745; Wagner et al. (2016), S. 742.

100 Vgl. Hubka (1984), S. 23 ff.

101 Vgl. Heidemann (2001), S. 21, 38; ebenso Hubka (1984), S. 17.

102 Vgl. Gramlich (2013), S. 64.
} 
Ein technischer Prozess beschreibt eine Zustandsänderung eines oder mehrerer Operanden in einem Zeitintervall. Der oder die Operanden werden dabei von einem Anfangszustand in einen Endzustand transformiert. Als Operanden fungieren ausschließlich Objekte, also stoffliche Gebilde. ${ }^{103}$

\section{Modelle technischer Prozesse}

Die im Rahmen der Produktentwicklung zur Verfügung stehenden Prozessmodelle ${ }^{104}$ bilden die Zustandsänderung von Objekten ab (siehe Abbildung 2-11). Das Modell gibt keinerlei Informationen darüber, auf welche Art und Weise der Prozess realisiert wird. ${ }^{105}$ Es werden lediglich der Anfangs- und der Endzustand des Operanden beschrieben.

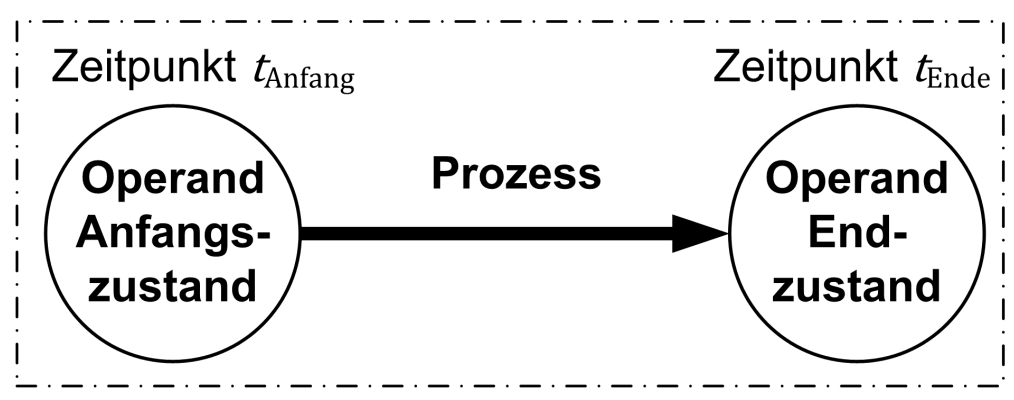

Abbildung 2-11: Zustandsänderung des Operanden im Prozess ${ }^{106}$

Im erweiterten Prozessmodell nach HEIDEMANN findet sich in Anknüpfung an HUBKA ${ }^{107}$ das technische Produkt als Arbeitsmittel wieder (siehe Abbildung 2-12). Mit Blick auf die Einsatzprozesse des Produkts stellt dieses, seiner Funktion folgend, eine Wirkgröße bereit, mittels derer die eigentliche Zustandsänderung der Operanden ${ }^{108}$ als passives Element aktiv eingeleitet und vorangetrieben wird. ${ }^{109}$

\footnotetext{
${ }^{103}$ Vgl. Gramlich (2013), S. 63 f.

104 Prozessmodelle stellen nach GRAMLICH zweckdienliche Abbildungen von Prozessen dar. (Vgl. Gramlich (2013), S. 165.)

105 Vgl. Gramlich (2013), S. 86.

106 Gramlich (2013), S. 64.

107 Vgl. Hubka (1984), S. 35.

108 HEIDEMANN verwendet die Begriffe Objekt und Größe im Gegensatz zum Verständnis in dieser Arbeit synonym, wodurch Größen bei inm auch stofflicher Natur sein können. (Vgl. Heidemann (2001), S. 39 f.)

109 Vgl. Heidemann (2001), S. $88 \mathrm{ff}$.
} 


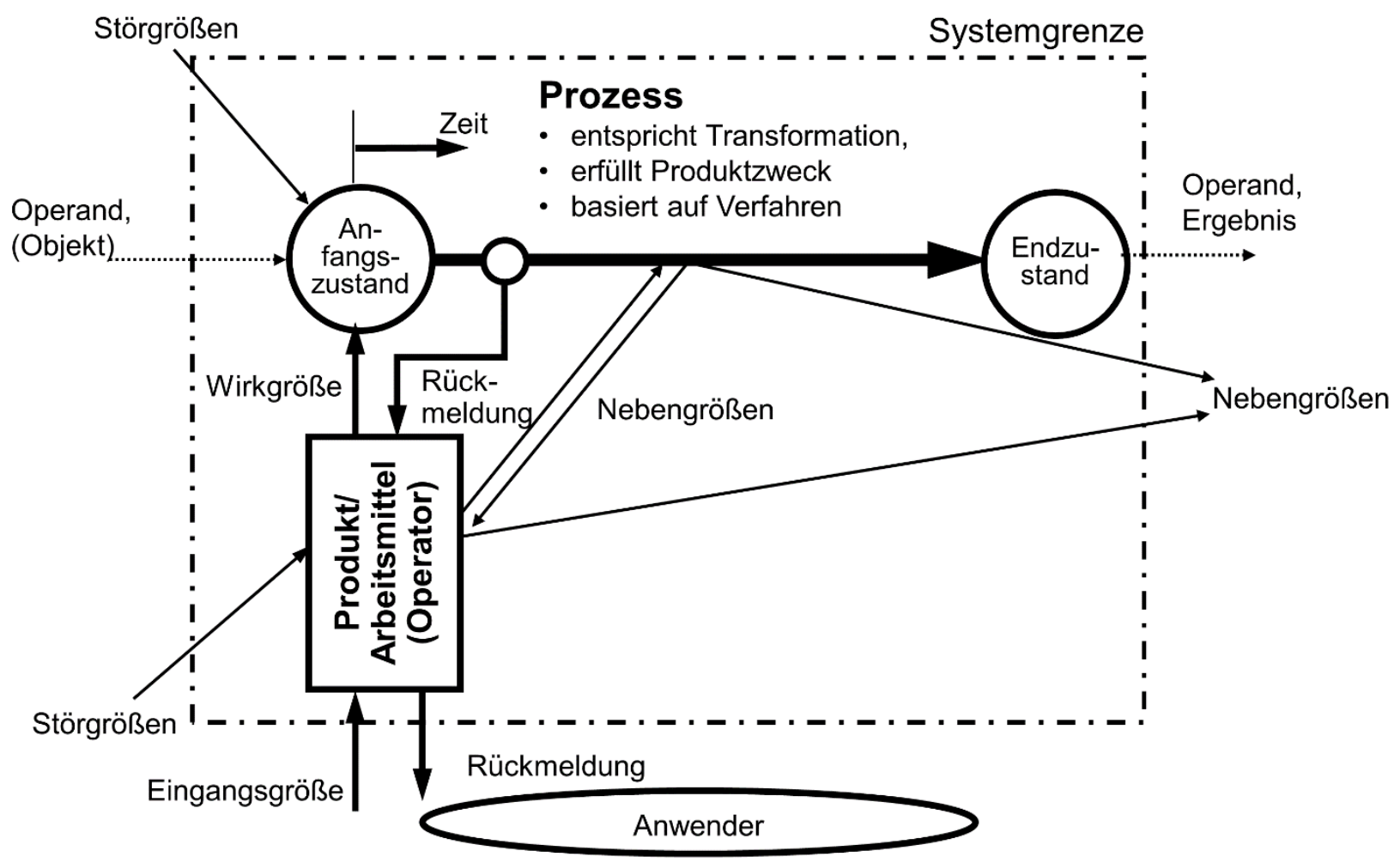

Abbildung 2-12: Erweitertes Prozessmodell nach HeIDEMANN ${ }^{110}$

Das Arbeitsmittel (Operator) erfährt selbst keine Zustandsänderung. Die Zustandsänderung des Operanden vom Anfangs- in den Endzustand ${ }^{111}$ erfolgt innerhalb einer definierten Systemgrenze. Elemente, die außerhalb der Systemgrenze liegen, tragen nicht direkt zur eigentlichen Zustandsänderung bei. Lediglich der Anwender kann durch Beeinflussung des Arbeitsmittels indirekt auf den Prozess einwirken. Zusätzlich bildet das Modell Stör- und Nebengrößen ab, die sowohl innerhalb der Systemgrenze als auch über sie hinweg ungewollte Einflüsse auf den Prozess bzw. vom Prozess auf die Umgebung darstellen. ${ }^{112}$ Insbesondere für komplexere Prozesse mit mehreren Operanden am Eingang bietet es sich an, diese durch Teilprozesse innerhalb einer Prozessstruktur abzubilden. Auf diese Weise können verschiedene parallel bzw. in Reihe geschaltete (Teil-)Prozesse abgebildet werden, die über definierte Zustände miteinander verknüpft sind. In der

\footnotetext{
110 Gramlich (2013), S. 32 in Anlehnung an Heidemann (2001), S. 138; begrifflich und grafisch angepasst nach Birkhofer (2011), S. 354.

111 HEIDEMANN verwendet synonym auch die Begriffe Eingang für Anfangszustand sowie Ausgang, Ergebnis und Prozessergebnis für den Endzustand (Vgl. Heidemann (2001), S. 77, 138.)

112 Vgl. Heidemann (2001), S. 76 ff.
} 
Prozessstruktur fungieren (Zwischen-)Zustände als Knoten, die durch Teilprozesse verbunden werden. ${ }^{113}$ Abbildung 2-13 zeigt die Teilprozesse zur Fertigung des bereits vorgestellten Posterständers unter Angabe der den Zustandsänderungen zugrunde liegenden Verfahren.

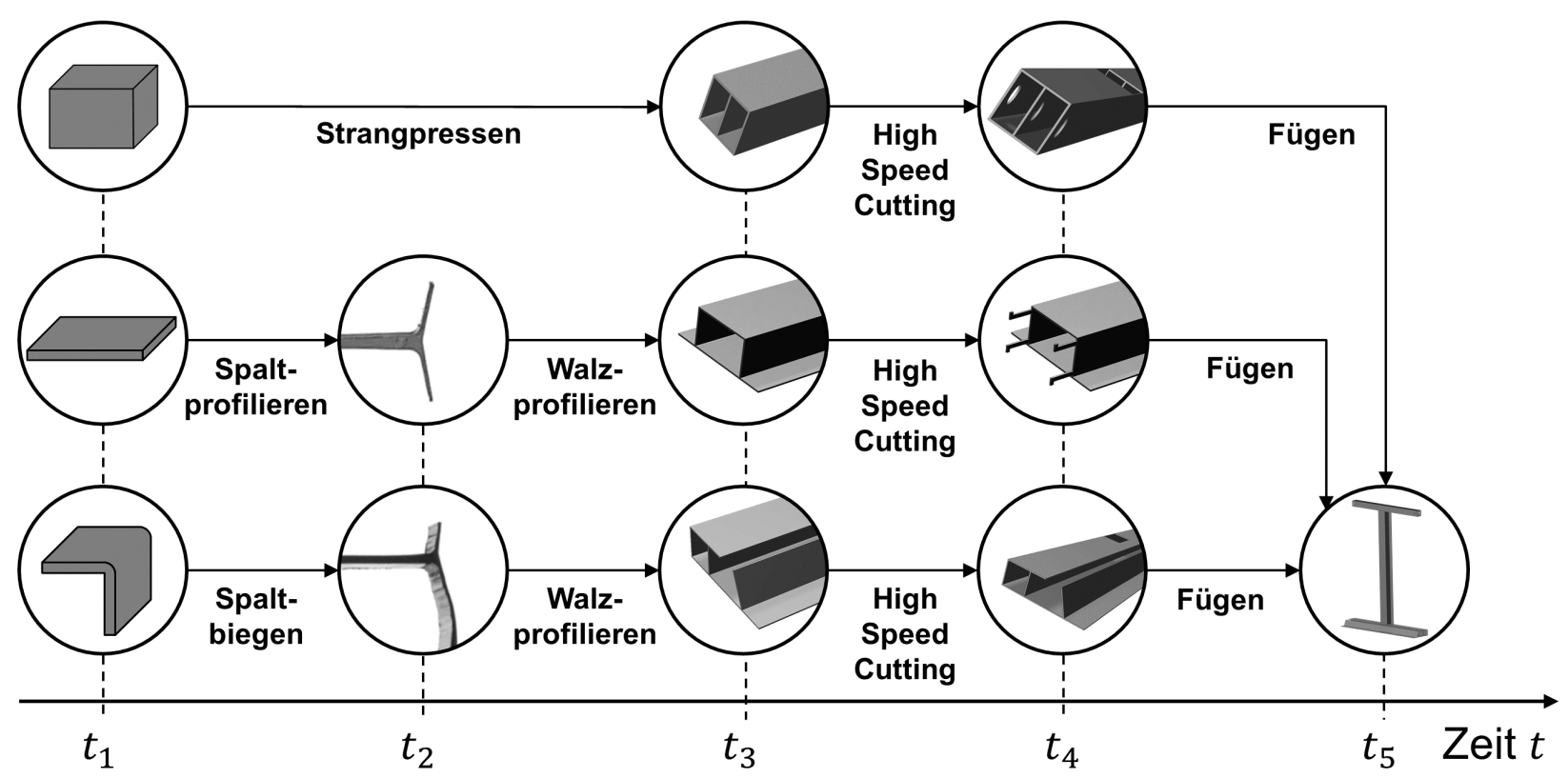

Abbildung 2-13: Prozessstruktur für die Fertigung eines Posterständers

\section{Verfahren und Verfahrensprinzip}

Verfahren geben Auskunft darüber, auf welche Art und Weise der technische Prozess abläuft. Sie erklären, wie die Zustandsänderung realisiert wird. ${ }^{114}$ Tabelle 2-3 gibt eine Übersicht gängiger Verfahrensdefinitionen. Ein Teil dieser Definitionen versteht unter Verfahren vor allem die für den technischen Prozess notwendigen Tätigkeiten, Operationen, Vorgänge, etc. und deren Abfolge. Diesem Begriffsverständnis liegt meist keine explizite Differenzierung der Begriffe Verfahren und Prozess zugrunde. HEIDEMANN fasst dies wie folgt zusammen: „Ein Verfahren identifiziert sich damit als Oberbegriff für eine Anzahl von Operationen in einer spezifischen Abfolge, wobei die einzelnen Operationen wieder aus Teilverfahren zusammengesetzt werden können." 115

\footnotetext{
113 Vgl. Heidemann (2001), S. $79 \mathrm{ff}$.

114 Vgl. Gramlich (2013), S. 86.

115 Heidemann (2001), S. 82.
} 
Tabelle 2-3: Übersicht gängiger Verfahrensdefinitionen

\begin{tabular}{|c|c|}
\hline Themengebiet & Definition \\
\hline Verfahrenstechnik & $\begin{array}{l}\text { „Unter einem Verfahren wird [...] die Kombination mehrerer } \\
\text { Grundoperationen zu dem Zweck, Materie (Stoff) nach Art, } \\
\text { Eigenschaft oder Zusammensetzung zu verändern, ver- } \\
\text { standen." }{ }^{116}\end{array}$ \\
\hline $\begin{array}{l}\text { Qualitäts- } \\
\text { management }\end{array}$ & $\begin{array}{l}\text { „festgelegte Art und Weise, eine Tätigkeit oder einen Pro- } \\
\text { zess [...] auszuführen“117 }\end{array}$ \\
\hline Fertigung & $\begin{array}{l}\text { „alle Verfahren zur Herstellung von geometrisch bestimm- } \\
\text { ten festen Körpern; sie schließen die Verfahren zur Gewin- } \\
\text { nung erster Formen aus dem formlosen Zustand, zur Ver- } \\
\text { änderung dieser Form sowie zur Veränderung der Stoffei- } \\
\text { genschaften ein“118 }\end{array}$ \\
\hline \multirow{5}{*}{ Produktentwicklung } & $\begin{array}{l}\text { "Als Verfahren bezeichnet man die Ausführung von Tätig- } \\
\text { keiten, Arbeitsabläufe, Stufen von Vorgängen oder } \underline{\text { Opera- }} \\
\text { tionsfolgen." }\end{array}$ \\
\hline & $\begin{array}{l}\text { "Verfahren realisieren Prozesse. Ein Verfahren bezeichnet } \\
\text { die geordnete Menge zweckentsprechend erforderlicher } \\
\text { Operationen technischer, manueller oder gedanklicher Na- } \\
\text { tur." }{ }^{120}\end{array}$ \\
\hline & $\begin{array}{l}\text { „Als Verfahren bezeichnet man die Abfolge von physika- } \\
\text { lisch-technischen, chemischen und biologischen Wirkungs- } \\
\text { abläufen, die zur Realisierung einer gewünschten Stoffän- } \\
\text { derung notwendig sind. Das Verfahren konkretisiert den } \\
\text { abstrakt formulierten Prozess, der die Transformation ei- } \\
\text { nes Operanden (Stoff) von einem Ausgangszustand in ei- } \\
\text { nen Endzustand beschreibt." }{ }^{21}\end{array}$ \\
\hline & $\begin{array}{l}\text { "Ein Verfahren ist stets ein komplexes Wirkungssystem } \\
\text { aus vielen Elementen, die untereinander und mit der Um- } \\
\text { gebung durch Stoff-, Energie-, und Informationsflüsse ver- } \\
\text { bunden sind." }{ }^{222}\end{array}$ \\
\hline & $\begin{array}{l}\text { „Verfahren werden als geordnete Abfolge von Wirkungsab- } \\
\text { läufen bzw. Operationen zur Realisierung einer Transfor- } \\
\text { mation eines oder mehrerer Operanden von einem An- } \\
\text { fangszustand in einen definierten Endzustand verstanden. } \\
\text { Durch die Festlegung eines Verfahrens wird die Realisie- } \\
\text { rung der als Zustandsänderung abstrakt beschriebenen } \\
\text { Prozesse konkretisiert." } 123\end{array}$ \\
\hline $\begin{array}{l}\text { Allgemeiner } \\
\text { Sprachgebrauch }\end{array}$ & $\begin{array}{l}\text { "Art und Weise der Durch-, Ausführung von etwas; Me- } \\
\text { thode“124 }\end{array}$ \\
\hline
\end{tabular}

116 Kögl, Moser (1981), S. 2; Hervorhebung durch den Verfasser.

117 DIN EN ISO 9000 (2015), S. 35; Hervorhebung durch den Verfasser. 
HEIDEMANN greift darüber hinaus einen Gedanken auf, der dem Verfahren eine gänzlich andere Bedeutung zugesteht: „Verfahren realisieren Prozesse“. ${ }^{125}$ Sie beschreiben somit, wie die durch die Zustandsänderung abstrakt beschriebenen Prozesse konkret realisiert werden. ${ }^{126}$ Eine Reihe neuerer Verfahrensdefinitionen, wie in DIN EN ISO 9000 zu finden, greift diesen Grundgedanken in ähnlicher Form auf. Verfahren umfassen demnach Angaben darüber, wie eine Tätigkeit auszuführen ist. Durch Gegenüberstellung mit den in DIN 8580 aufgeführten Fertigungsverfahren zeigt sich, dass $u$. a. Angaben zu Arbeitsmitteln und deren Verwendung benötigt werden, um die Art und Weise der Zustandsänderung zu beschreiben.

Verfahren beschreiben die geordnete Abfolge von Operationen, die notwendig sind, um einen Prozess zu realisieren. Verfahren definieren, in welcher Art und Weise die Zustandsänderung abläuft. ${ }^{127}$

\section{Verfahrensprinzip}

Das Verfahren beinhaltet gemäß der Definition von GRAMLICH Aussagen über Wirkungsabläufe die auf physikalischen Gesetzmäßigkeiten (physikalischen Effekten) beruhen. „Diese Gesetzmäßigkeiten werden durch Festlegen von Wirkgrößen sowie geometrischen und stofflichen Eigenschaften der am Prozess beteiligten bzw. für die Transformation relevanten stofflichen Systeme in einen Wirkzusammenhang gebracht, der durch das Verfahrensprinzip beschrieben ist." ${ }^{128}$

Das Verfahrensprinzip eines Verfahrens beschreibt den Wirkzusammenhang zwischen Wirkelementen und Wirkgrößen, die an der Realisierung der Zustandsänderung des Operanden beteiligt sind. ${ }^{129}$

\footnotetext{
${ }^{118}$ DIN 8580 (2003), S. 4.

119 Rodenacker (1991), S. 47; Hervorhebung durch den Verfasser.

120 Heidemann (2001), S. 45; Hervorhebung durch den Verfasser.

${ }^{121}$ Sauer (2006), S. 74; Hervorhebung durch den Verfasser.

122 VDI 2221 (1993), S. 24.

${ }^{123}$ Gramlich (2013), S. 167; Hervorhebung durch den Verfasser.

124 Dudenredaktion (2017b); Hervorhebung durch den Verfasser.

${ }^{125}$ Heidemann (2001), S. 45.

${ }^{126}$ Vgl. Sauer (2006), S. 70.

127 Vgl. Gramlich (2013), S. 167; Heidemann (2001), S. 45; DIN EN ISO 9000 (2015), S. 35.

${ }^{128}$ Gramlich (2013), S. 87.

${ }^{129}$ Vgl. Gramlich (2013), S. 87.
} 
Das Verfahrensprinzip beschreibt das Verfahren, also die Art und Weise des Ablaufs der Zustandsänderung, mittels des Zusammenwirkens von Arbeitsmittel und Operanden. Das Arbeitsmittel wird durch verfahrensprinziprelevante Wirkelemente beschrieben, die für die Durchführung des technischen Prozesses relevant sind. Verfahrensprinziprelevante Wirkelemente können auch dem Operanden zugeordnet sein. Sie umfassen Wirkflächen, Wirkkörper oder Wirkräume. In Übereinstimmung mit der Differenzierung von Objekten und Größen sind verfahrensprinziprelevante Wirkelemente stofflicher Natur. Die während der Zustandsänderung auf den Operanden einwirkenden Wirkgrößen sind ausschließlich Energien oder Signale. ${ }^{130}$

Das Verfahrensprinzip des Spaltprofilierens beschreibt das Zusammenwirken einer Spaltwalze (Arbeitsmittel) und zwei Hilfswalzen (Arbeitsmittel) mit einem Blechhalbzeug (Operand) (siehe Abbildung 2-14). Unter einer Kraft $F$ (Wirkgröße) auf die Blechkante, werden zwei Flansche ausgeformt. Das Blech wird mit der Geschwindigkeit $v_{\text {Blech }}$ durch das Werkzeugsystem befördert. Während der Umformung fließt das durch die um $y_{\text {ink }}$ zugestellte Spaltwalze verdrängte Halbzeugvolumen durch die Freiräume zwischen Spaltwalze und Hilfswalze, wodurch sich die charakteristischen Flansche ausbilden.

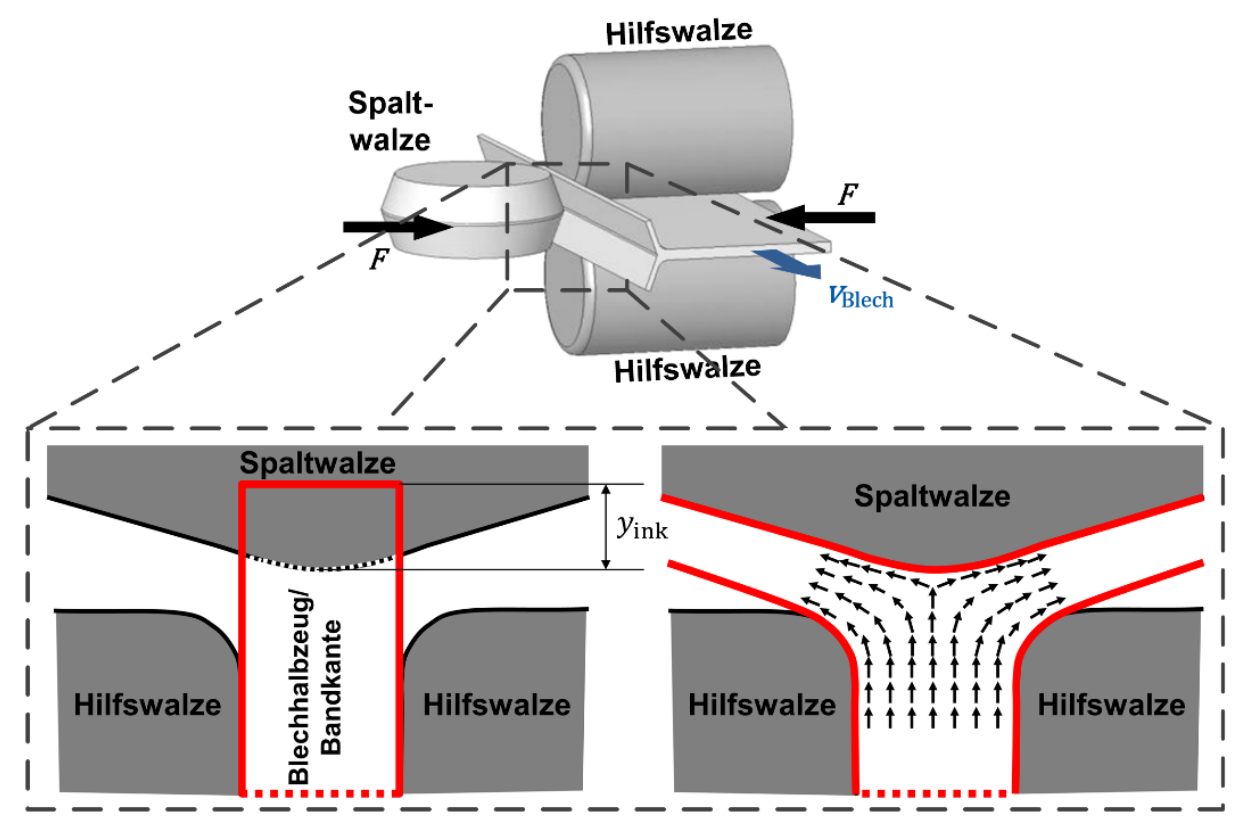

Abbildung 2-14: Verfahrensprinzip Spaltprofilieren ${ }^{131}$

\footnotetext{
130 Vgl. Gramlich (2013), S. 87.

131 Gramlich (2013), S. 88 in Anlehnung an Bruder (2011), S. 16; Groche et al. (2012b), S. 87.
} 
Dem Innenhochdruckumformen liegt ein gänzlich anderes Verfahrensprinzip zugrunde. Die Arbeitsmittel, bestehend aus Oberwerkzeug, Unterwerkzeug und Axialstempeln, umschließen das umzuformende rohrförmige Halbzeug (Operand) komplett (siehe Abbildung 2-15). Zusätzlich ist das Rohr mit einem Wirkmedium gefüllt, das ebenfalls zum Arbeitsmittel zu zählen ist. Über dieses wird ein Innendruck $p_{\mathrm{i}}$ als Wirkgröße auf das Rohr ausgeübt, der letztlich zur Umformung des Rohres führt. Das Rohr legt sich dabei an die Innenkontur des Ober- und Unterwerkzeugs an und erhält somit seine Außengeometrie im Endzustand. Zusätzlich werden eine Schließkraft $F_{\text {schließ }}$ und eine Axialkraft $F_{\text {axial }}$ als Wirkgrößen benötigt, um zu gewährleisten, dass das Werkzeug auch während der Umformung des Rohres geschlossen bleibt. Durch das Zusammenwirken der beschriebenen Elemente wird ein Rohr erzeugt, das durch eine Geometrie mit unterschiedlichen Querschnitten über der Rohrlänge gekennzeichnet ist.

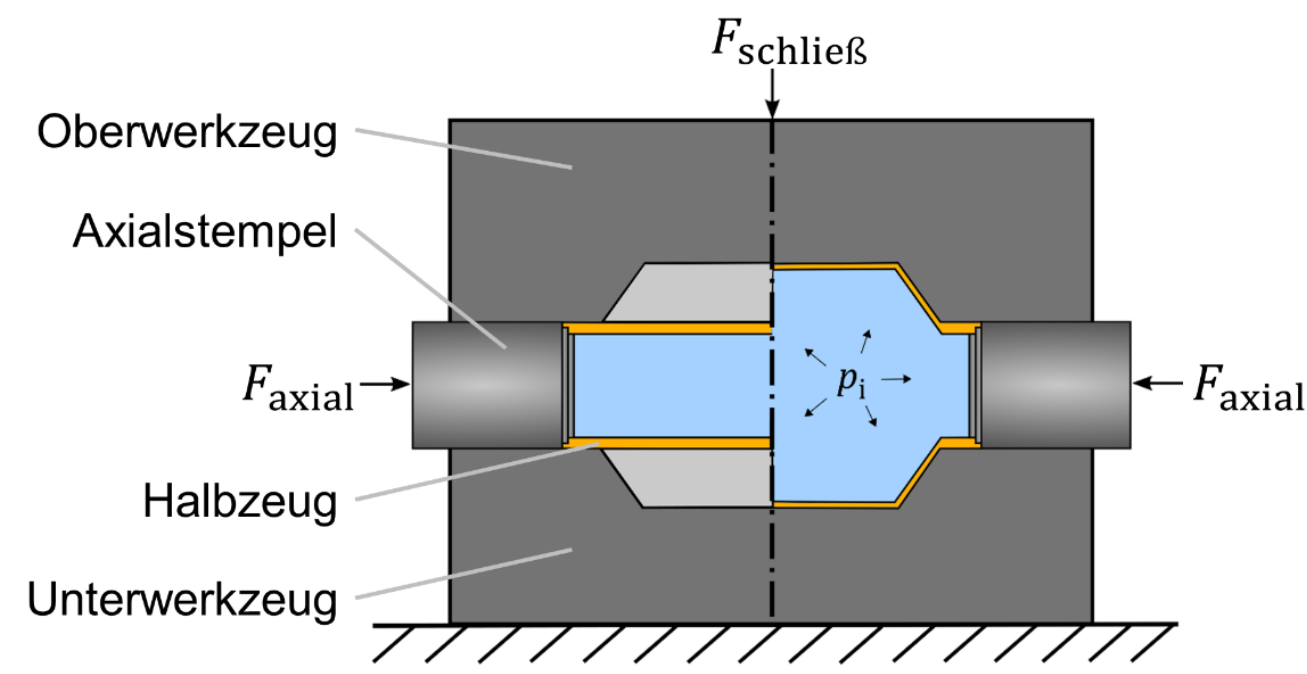

Abbildung 2-15: Verfahrensprinzip Innenhochdruckumformen ${ }^{132}$

\section{Beschreibung technischer Prozesse durch Eigenschaften}

HUBKA beschreibt, dass die Eigenschaften des Operanden durch den Transformationsprozess geändert werden. ${ }^{133}$ Jeder Zustand, den der Operand bzw. das Objekt zu bestimmten Zeitpunkten durchläuft, kann über eine Menge von Eigenschaften beschrieben werden. In Abbildung 2-16 werden diese in Form von Eigenschaftsvektoren dargestellt. Die Zustände unterscheiden sich dabei in mindestens einer Eigenschaft. ${ }^{134}$

\footnotetext{
132 In Anlehnung an Noack, Sterzing (2007), S. 15 und VDI 3146-1 (1999).

${ }^{133}$ Vgl. Hubka (1984), S. 23.

${ }^{134}$ Vgl. Gramlich (2013), S. 64.
} 


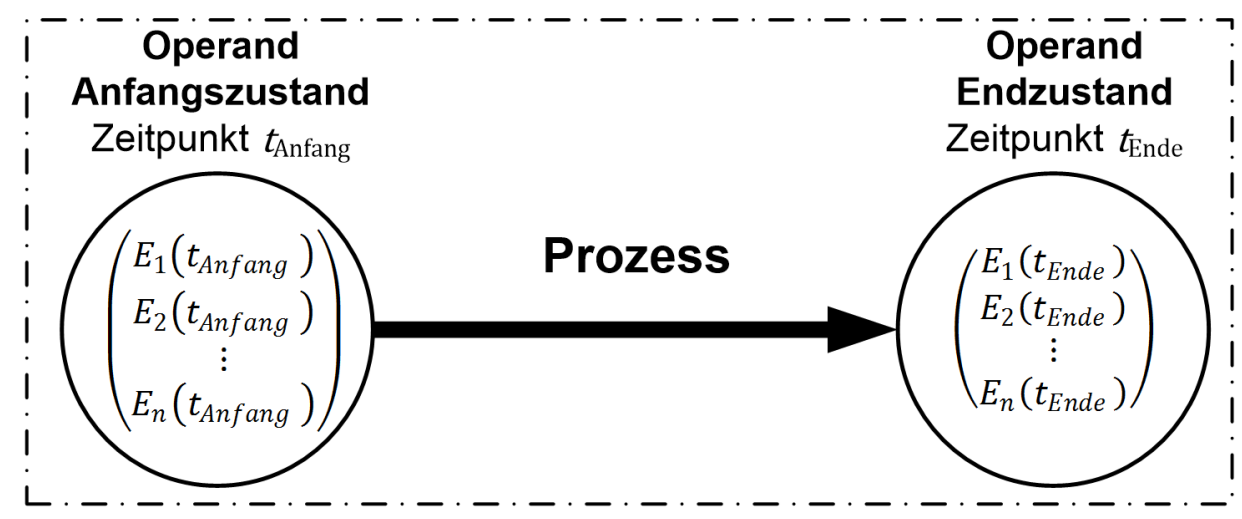

Abbildung 2-16: Beschreibung der Objektzustände mittels Eigenschaften ${ }^{135}$

GRAMLICH beschreibt die durch das Verfahren charakteristischerweise hervorgerufenen Eigenschaften als verfahrensinduzierte Eigenschaften ${ }^{136}$. Diese umfassen alle Eigenschaften des Operanden im Endzustand, die durch Verwendung eines bestimmten Verfahrens geändert wurden. Somit umfassen diese nicht alle Eigenschaften des Objekts im Endzustand, sondern nur diejenigen, auf die im Zuge der Zustandsänderung aktiv Einfluss genommen wurde. Die verfahrensinduzierten Eigenschaften sind stets verfahrensinduzierten Gestaltelementen zugeordnet, die ebenso charakteristisch durch ein Verfahren erzeugt werden. Die verfahrensinduzierten Eigenschaften sind daher Eigenschaften, die die Gestalt des Objekts beschreiben. Sie sind geometrischer und werkstofflicher Natur. ${ }^{137}$ Verfahrensinduzierte Eigenschaften und verfahrensinduzierte Gestaltelemente charakterisieren somit das verwendete Verfahren. ${ }^{138}$ Für das Beispiel spaltprofilierter Bauteile stellen die spaltprofilierten Flansche verfahrensinduzierte Gestaltelemente dar (siehe Abbildung 2-17). Sie werden durch eine Vielzahl an Eigenschaften beschrieben, zu denen insbesondere die hohe Härte, die hohe Festigkeit, die Flanschlängen sowie die ultrafeinkörnige (UFG) Gefügestruktur zählen.

Die verfahrensprinziprelevanten Wirkelemente des Arbeitsmittels werden durch verfahrensprinziprelevante Eigenschaften beschrieben. ${ }^{139}$ Am Beispiel des Spaltprofilierens sind dies u. a. die Durchmesser der einzelnen Spalt- und Hilfswalzen oder die Radien an deren Außenkontur. Auch die Steifigkeit der Walzen hat als

135 Gramlich (2013), S. 64, 65.

${ }^{136}$ In der Literatur finden sich ebenso die Begriffe technologieinduzierte Eigenschaften (vgl.

Groche et al. (2012b)), technology-induced properties (vgl. Groche et al. (2012b)) und manufacturing-induced properties (vgl. Groche et al. (2012a)).

${ }^{137}$ Vgl. Gramlich (2013), S. $90 \mathrm{ff}$.

138 Vgl. Gramlich (2013), S. 116.

139 Vgl. Gramlich (2013), S. 87 ff. 
verfahrensprinziprelevante Eigenschaft des Arbeitsmittels einen entsprechenden Einfluss auf die Umformung. Die verfahrensprinziprelevanten Wirkelemente des Operanden im Anfangszustand werden ebenso durch verfahrensprinziprelevante Eigenschaften beschrieben. Am Beispiel des Spaltprofilierens ist eine hohe Duktilität, eine hohe Festigkeit sowie eine glatte, gerade und rechtwinklige Bandkante vorzusehen. Die Spaltprofilieranlage, zu der das Werkzeugsystem mit den Spaltund Hilfswalzen zählt, stellt wiederum als Wirkgrößen die Kraft $F$ und den Bandvorschub $v_{\text {Blech }}$ bereit, die formal wie Eigenschaften beschrieben werden können.

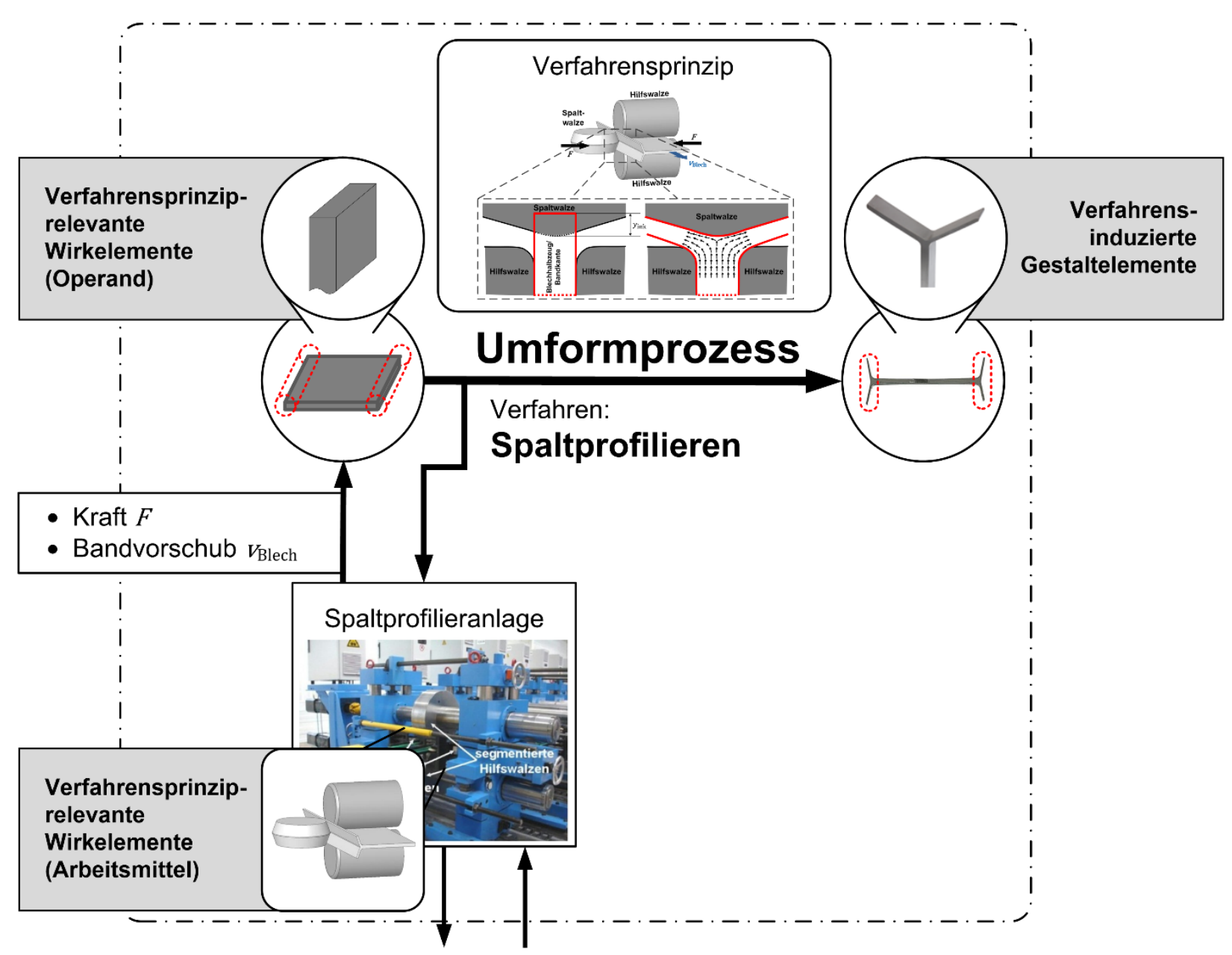

Abbildung 2-17: Modellelemente des Verfahrensprinzips am Beispiel des Spaltprofilierens ${ }^{140}$

140 In Anlehnung an Gramlich (2013), S. 98. 


\subsubsection{Modellierung des Produktlebenslaufs}

Die Summe aller Prozesse, die ein Produkt in seinem Leben durchläuft, werden im Produktlebenslauf zusammengefasst (siehe Abbildung 2-18). Er ist in vier Phasen unterteilt: Werkstoffherstellung, Produktion, Nutzung und Recycling/Entsorgung. Mit Ausnahme der Nutzung dient das Produkt als Operand der technischen Prozesse. In der Nutzungsphase wechselt es zur Rolle des Arbeitsmittels. ${ }^{141}$

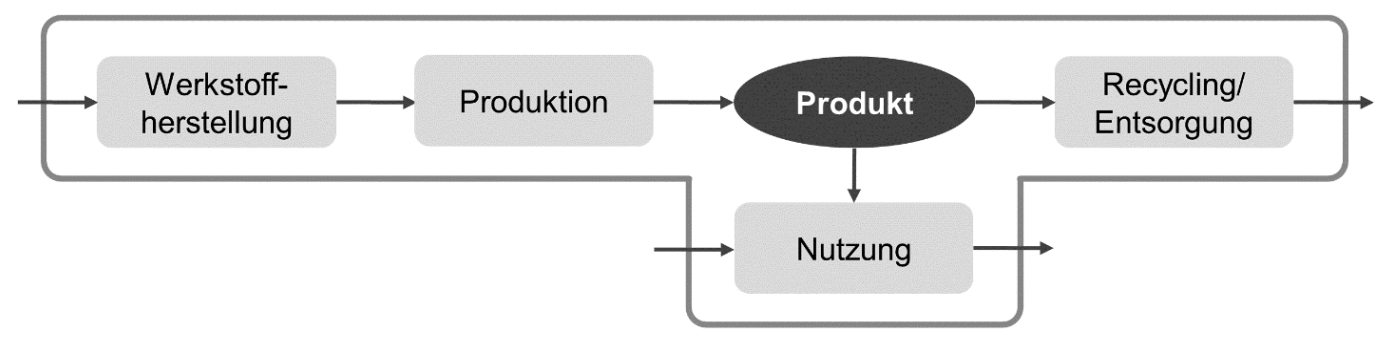

Abbildung 2-18: Lebenslaufphasen eines technischen Produkts ${ }^{142}$

Die Nutzungsphase kann zudem in Nutzungsteilphasen gegliedert werden (Abbildung 2-19). In der Teilphase Einsatz fungiert das Produkt als Arbeitsmittel der zugehörigen technischen Prozesse. Es stellt selbst die Wirkgröße für die ablaufenden Zustandsänderungen bereit. Diese Teilphase ist ausschlaggebend für die Sonderrolle der Nutzung im Produktlebenslauf. ${ }^{143}$ In den Prozessen der übrigen Teilphasen ist das Produkt wiederum der Operand. Sie werden fertigungsanaloge Nutzungsprozesse genannt, da sie wie Fertigungsprozesse modelliert werden. ${ }^{144}$

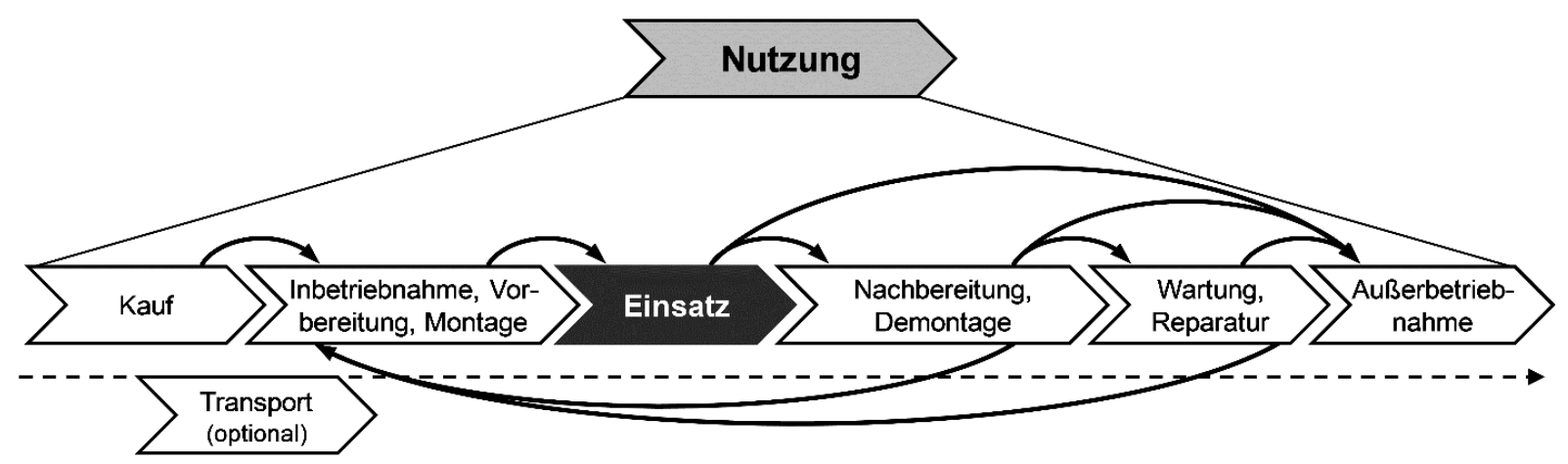

Abbildung 2-19: Nutzungsteilphasen eines technischen Produkts ${ }^{145}$

\footnotetext{
${ }^{141} \mathrm{Vgl}$. Birkhofer et al. (2012), S. $564 \mathrm{f}$.

${ }^{142}$ Birkhofer et al. (2012), S. 564.

${ }^{143}$ Vgl. Dannheim (1999), S. 46; Oberender (2006), S. 33 ff.

${ }^{144} \mathrm{Vgl}$. Gramlich (2013), S. 104.

145 Gramlich (2013), S. 104 in Anlehnung an Dannheim (1999), S. 46 und Oberender (2006), S. 34.
} 
Für Bauteilverbindungen ist es erforderlich die Begriffe Montage und Fügeprozess zu differenzieren, um die entsprechenden Lebenslaufprozesse eindeutig zu benennen. Fügen ist gemäß DIN 8580 wie folgt definiert.

Fügen ist ein „auf Dauer angelegtes Verbinden oder sonstiges Zusammenbringen von zwei oder mehreren Werkstücken geometrisch bestimmter fester Form oder von eben solchen Werkstücken mit formlosem Stoff; dabei wird der Zusammenhalt örtlich geschaffen und im Ganzen vermehrt“. ${ }^{146}$

Gemäß dieser Definition existieren Fügeverfahren, die entsprechende Fügeprozesse realisieren. Die Montage wird demgegenüber wie folgt abgegrenzt.

Montieren wird als „die Gesamtheit aller Vorgänge [verstanden], die dem Zusammenbau von geometrisch bestimmten Körpern dienen." 147

Sie umfasst Fügeprozesse sowie Prozesse zur Werkstückhandhabung, zum Kontrollieren, zum Justieren und Sonderoperationen. Die Montage ist somit ein übergeordneter Begriff für die Gesamtheit der Prozesse bzw. Vorgänge, die über die gewollte Zustandsänderung des Operanden hinaus erledigt werden müssen. ${ }^{148}$

\subsubsection{Integrale Ansätze zur Berücksichtigung der Fertigung während der Produktentwicklung}

Zur Strukturierung des Vorgehens während der Produktentwicklung findet sich in der Literatur eine Reihe unterschiedlicher Vorgehensmodelle. Diese setzen sich stets aus aufeinanderfolgenden oder parallelisierten Schritten bzw. Aktivitäten zusammen, die während der Entwicklung durchlaufen werden. ${ }^{149} \mathrm{Im}$ englischsprachigen Raum ist das Axiomatic Design ${ }^{150}$ weitverbreitet. Das Vorgehen wird durch vier horizontal angeordnete Domains strukturiert, in denen das Produkt schrittweise konkretisiert wird: Customer Domain, Functional Domain, Physical Domain und Process Domain. Die letzte Domain ist der Herstellung gewidmet. Hier werden

\footnotetext{
146 DIN 8580 (2003), S. 5.

147 Lotter, Wiendahl (2006), S. 2 in Anlehnung an VDI 2860 (1990).

$148 \mathrm{Vgl}$. Lotter, Wiendahl (2006), S. 2.

${ }^{149} \mathrm{Vgl}$. Lindemann (2009), S. 57.

150 Vgl. Suh (1998).
} 
Process Variables definiert, die die Prozesse und Ressourcen beschreiben, die zur Herstellung des Produkts benötigt werden. ${ }^{151}$ Fertigungstechnologisches Wissen wird hier lediglich genutzt, um die Herstellbarkeit der Produkte im Kontext der Process Domain sicherzustellen. TEKKAYA zeigt, dass gerade beim Übergang von der Physical Domain zur Process Domain erhebliche Iterationen notwendig werden, um die Herstellbarkeit der festgelegten Produktgestalt zu erreichen. ${ }^{152}$

Im deutschsprachigen Raum sind die Vorgehensmodelle nach VDI $2221^{153}$ und VDI $2206^{154}$ weitverbreitet. Die einzelnen Konkretisierungsschritte werden in die Phasen Projektdefinition, Konzept, Entwurf und Ausarbeitung gegliedert und durch geeignete Methoden und Werkzeuge unterstützt. ${ }^{155}$ Die Partialmodelle der Produktmodellpyramide korrelieren mit dem, während der Entwicklung zunehmenden, Konkretisierungsgrad der Lösung. ${ }^{156}$ Fertigungsaspekte werden im Rahmen dieser Vorgehensmodelle meist erst in den späten Entwicklungsphasen z. B. durch Anwendung von DfM-Werkzeugen berücksichtigt.

Aufbauend auf dem Grundgedanken der VDI 2221 finden sich vielzählige Abwandlungen und Erweiterungen dieses Vorgehensmodells. Unter anderem ist hier das Modell der ganzheitlichen Produkt- und Prozessentwicklung (GPPE) ${ }^{157}$ zu nennen. Es vereint die Prozesskette der Produktentwicklung mit dem Produktlebenslauf (siehe Abbildung 2-20). Die Teilphasen der Produktentwicklung basieren im Wesentlichen auf den Phasen, wie sie bereits in der VDI 2221 angegeben werden. An deren Ende steht das Produkt, dessen geometrische und werkstoffliche Eigenschaften vollständig festgelegt sind. Diese sind die Grundlage für die Produktion und damit die physische Realisierung des Produkts. Das entscheidende Modellelement der GPPE stellt das Antizipieren von Lebenslaufprozessen dar, da es sich in dieser Form weder in konventionellen Entwicklungsansätzen noch in den vorgestellten Ansätzen des DfM wiederfindet. Ziel dabei ist es, Lebenslaufprozesse, wie die Fertigung, bereits während der Produktentwicklung vorauszudenken und entsprechende Informationen bei der Konkretisierung des Produkts zu

\footnotetext{
151 Vgl. Suh (1998), S. 204 f.

152 Vgl. Tekkaya et al. (2015), S. 647.

153 Vgl. VDI 2221 (1993).

154 Vgl. VDI 2206 (2004).

155 Vgl. Birkhofer (2011), S. 335.

156 Vgl. Wäldele (2012), S. 28 ff.

${ }^{157}$ Vereinzelt findet sich in der Literatur auch der Begriff T-Modell, da die grafische Darstellung des Modells einem auf den Kopf gestellten „T“ ähnelt.
} 
berücksichtigen. Die Schlagkraft dieses Ansatzes liegt darin, Informationen sämtlicher Lebenslaufprozesse gleichermaßen der Produktentwicklung zur Verfügung zu stellen. Das führt jedoch zu dem Schluss, dass während der Produktentwicklung nicht nur das Produkt konkretisiert und festgelegt wird, sondern auch die einzelnen Prozesse des Lebenslaufs beeinflusst werden. ${ }^{158}$

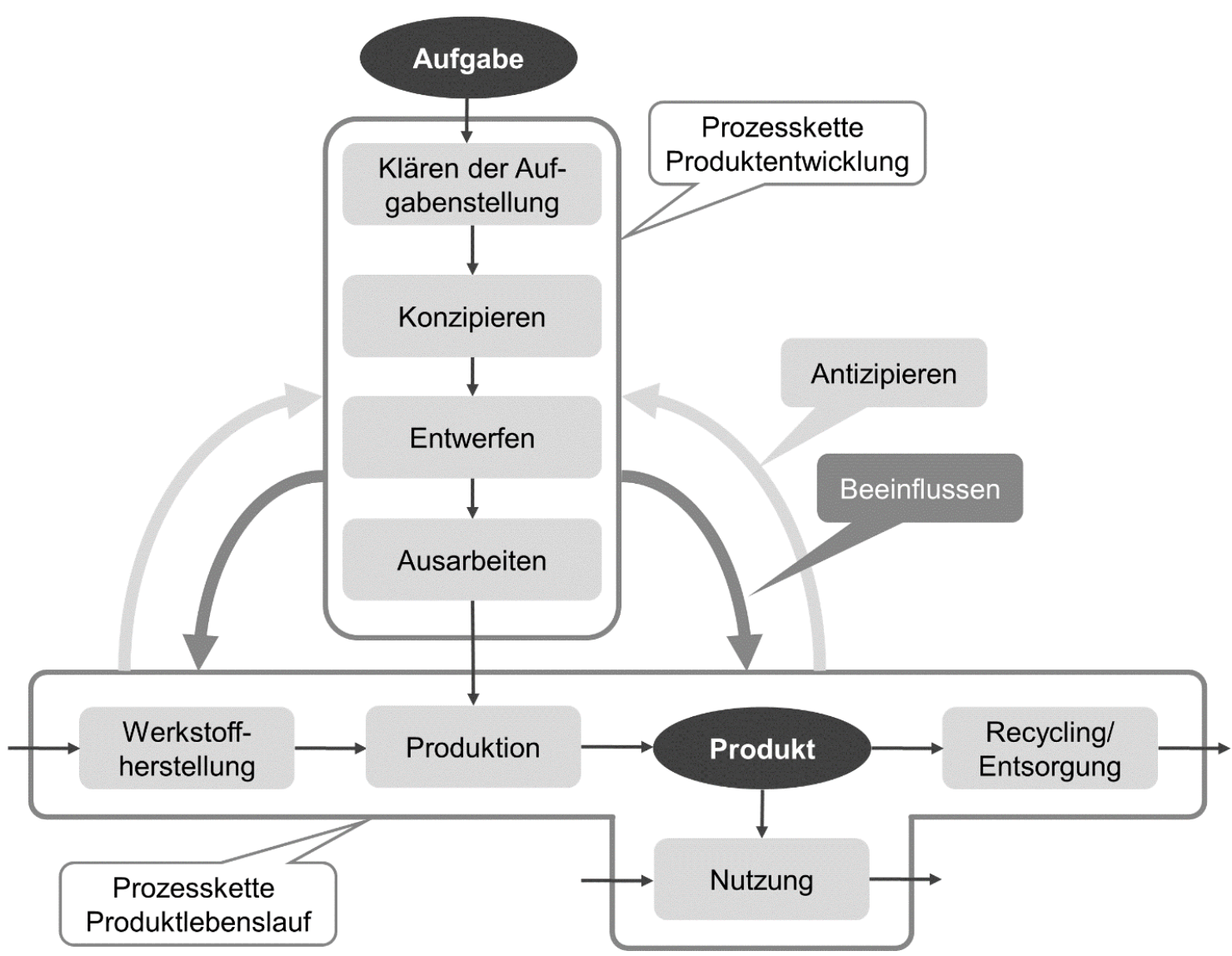

Abbildung 2-20: Modell der ganzheitlichen Produkt- und Prozessentwicklung ${ }^{159}$

Eine besondere Stellung unter den Entwicklungsansätzen nimmt die produktionsintegrierende Entwicklungsmethodik nach GRAMLICH ein. Sie basiert auf der konsequenten Modellierung technischer Produkte und Prozesse mittels Eigenschaften, um ganzheitliche Produktinnovation zu generieren. ${ }^{160}$ Durch diesen Ansatz ist es möglich, verfahrensinduzierte Eigenschaften und Gestaltelemente einheitlich aufzubereiten und dem Entwicklungsprozess zugänglich zu machen. Kernbestandteil der Methodik ist ein systematischer Abgleich von verfahrensinduzierten

\footnotetext{
${ }^{158}$ Vgl. Birkhofer, Schott (1996), S. 386 ff.; Schott (1998), S. 38 ff.; Grüner (2001), S. 45 ff.; Abele et al. (2008), S. 5 f.; Birkhofer et al. (2007), S. 206 f.; Birkhofer (2011), S. 348 f.

159 Grüner (2001), S. 46; Birkhofer et al. (2012), S. 566.

${ }^{160}$ Vgl. Gramlich (2013), S. 3, 117 ff.
} 
Eigenschaften mit wirkprinziprelevanten ${ }^{161}$ und verfahrensprinziprelevanten Eigenschaften im Rahmen eines sogenannten Mappings. ${ }^{162}$ Durch Verwendung des Mappings in marktgetriebenen Produktsynthesestrategien kann eine Produktgestalt generiert werden, die die Möglichkeiten der gewählten Fertigungstechnologien gezielt ausschöpft. Zudem können Fertigungsverfahren in Übereinstimmung mit dem Wirkprinzip des technischen Produkts frühzeitig im Entwicklungsprozess festgelegt werden (siehe Abbildung 2-21).

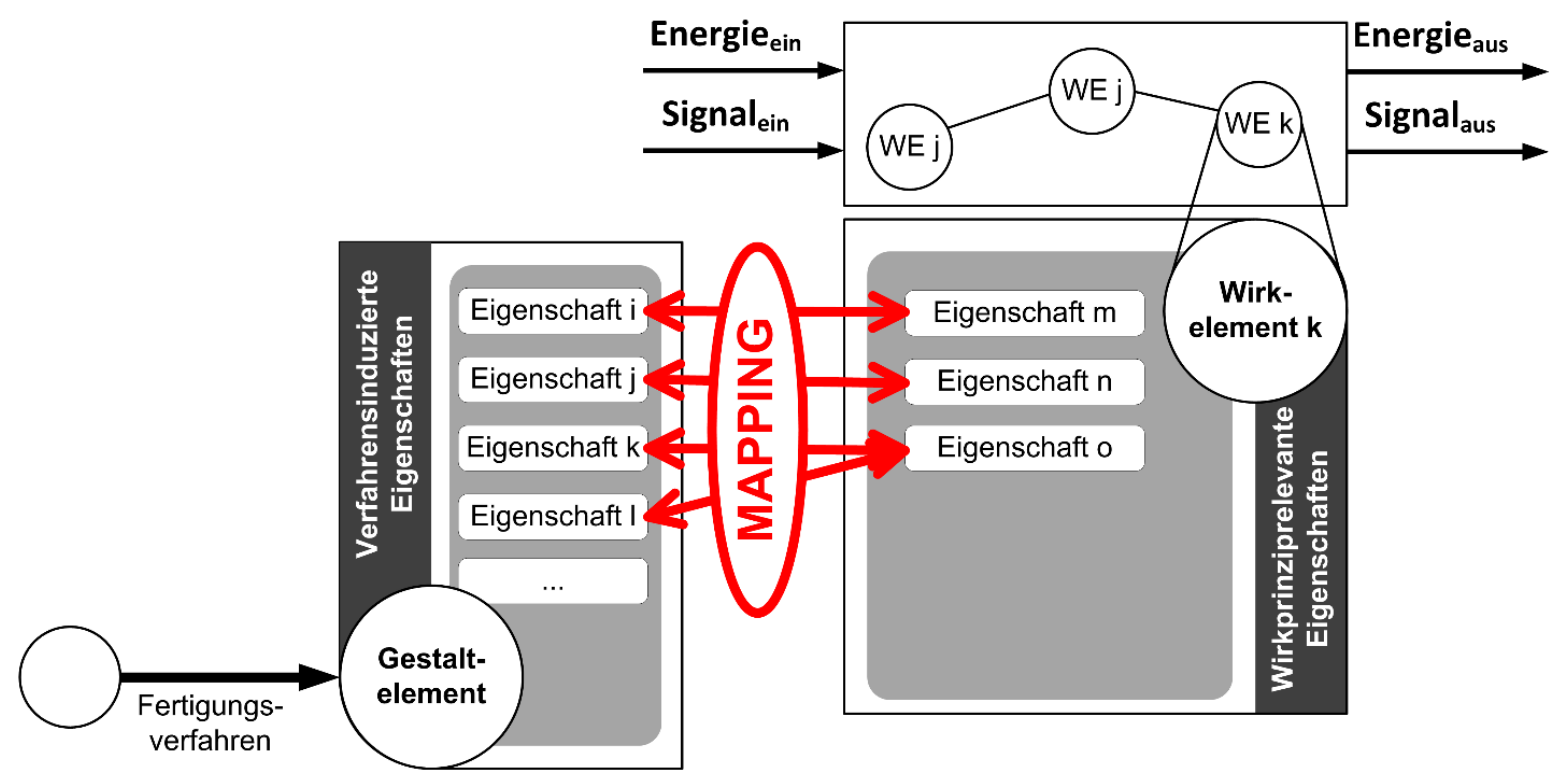

Abbildung 2-21: Marktgetriebenes Mapping zum Festlegen geeigneter Fertigungsverfahren ${ }^{163}$

Die produktionsintegrierende Entwicklungsmethodik nach GRAMLICH unterstützt gezielt die gleichwertige Verarbeitung von Fertigungsaspekten und Produktaspekten auf der Grundlage einer einheitlichen Modellierung mittels Eigenschaften. Die vorgestellten Ansätze können fertigungstechnologisches Wissen jedoch noch nicht umfassend für die Produktentwicklung nutzbar machen. Der vorgestellte Abgleich von Eigenschaften liefert Aussagen über die Kompatibilität von Fertigungsverfahren mit dem Produkt. Darüber hinaus ist es jedoch notwendig, gezielt Verknüpfungen zwischen fertigungstechnologischem Wissen und dem Produkt her-

\footnotetext{
${ }^{161}$ Wirkprinziprelevante Eigenschaften, sind die Produktmodelleigenschaften, die für die Umsetzung des Wirkprinzips unabdingbar sind. (Vgl. Gramlich (2013), S. 85.)

162 Vgl. Gramlich (2013), S. 124 ff. An anderer Stelle wird auch der Begriff Matching für das Eigenschafts-Mapping verwendet: Lüthen et al. (2017), S. 159 ff.

163 In Anlehnung an Gramlich (2013), S. 127.
} 
zustellen, um neuartige Lösungsmöglichen für die Produktentwicklung zu erschließen. Die konsistente Modellierung von Produkten und Prozessen mittels Eigenschaften bietet eine geeignete Grundlage, um darauf aufbauend eine noch engere Verknüpfung von Produkt- und Prozessmodellierung zu erreichen und Verknüpfungen zwischen fertigungstechnologischem Wissen und dem Produkt herauszuarbeiten. Dies erfordert weitere Aufarbeitungsschritte im Rahmen einer übergeordneten Methodik.

\subsection{Generelles Bereitstellen und Nutzen von Wissen durch Wissensmanagementsysteme}

Fertigungstechnologisches Wissen wurde als Schlüsselelement für die Lösung technischer Problemstellungen identifiziert. ${ }^{164}$ Auch auf Unternehmensebene wird Wissen als entscheidender Produktions- und Wettbewerbsfaktor verstanden, dem im strategischen und operativen Management besondere Aufmerksamkeit geschenkt werden muss. ${ }^{165}$ In diesem Kontext haben sich verschiedene Wissensmanagementsysteme herausgebildet, deren Ziel die Planung, Steuerung, Organisation und Kontrolle aller Prozesse von der Wissensgenerierung bis hin zur Wissensbereitstellung ist. ${ }^{166}$ Die folgenden Ausführungen beziehen sich exemplarisch auf das „Wissensmanagement-Referenzmodell“167. Dieses basiert auf dem Gedanken, die große Zahl bestehender Wissensmanagementsysteme möglichst umfassend in einem Referenzmodell zusammen zu führen. Das daraus resultierende Modell (siehe Abbildung 2-22) stellt die wertschöpfenden Geschäftsprozesse ins Zentrum der Betrachtungen. Sie stellen auf der einen Seite die Wissensquelle dar und liefern auf der anderen Seite den Anwendungskontext des Wissens.

Um die Geschäftsprozesse drehen sich die Kernaktivitäten, Wissen zu erzeugen, zu speichern, zu verteilen und anzuwenden. In der Aktivität „Wissen erzeugen“ wird das Wissen entweder intern entwickelt oder aus dem Umfeld des Unternehmens bezogen. In der darauffolgenden Aktivität wird das Wissen identifiziert, selektiert und personen- oder dokumentgebunden gespeichert. Das Verteilen des Wissens hat zum Ziel das Wissen an den Stellen im Unternehmen zur Verfügung zu stellen, an denen es tatsächlich gebraucht wird. Durch Anwenden des Wissens

\footnotetext{
${ }^{164} \mathrm{Vgl}$. Pahl et al. (2007), S. 1.

165 Vgl. Rehäuser, Krcmar (1996), S. 18.

166 Vgl. Rehäuser, Krcmar (1996), S. 10.

167 Vgl. Probst et al. (2012).
} 
soll die Problemlösung unterstützt werden. Um Kernaktivitäten ordnen sich sechs Gestaltungsfelder an: Unternehmenskultur, Personal, Controlling, Organisation, Informationstechnik und Führungssysteme. Ihr Vorhandensein dient der Sensibilisierung der Managementaktivitäten der einzelnen Unternehmensbereiche. ${ }^{168}$

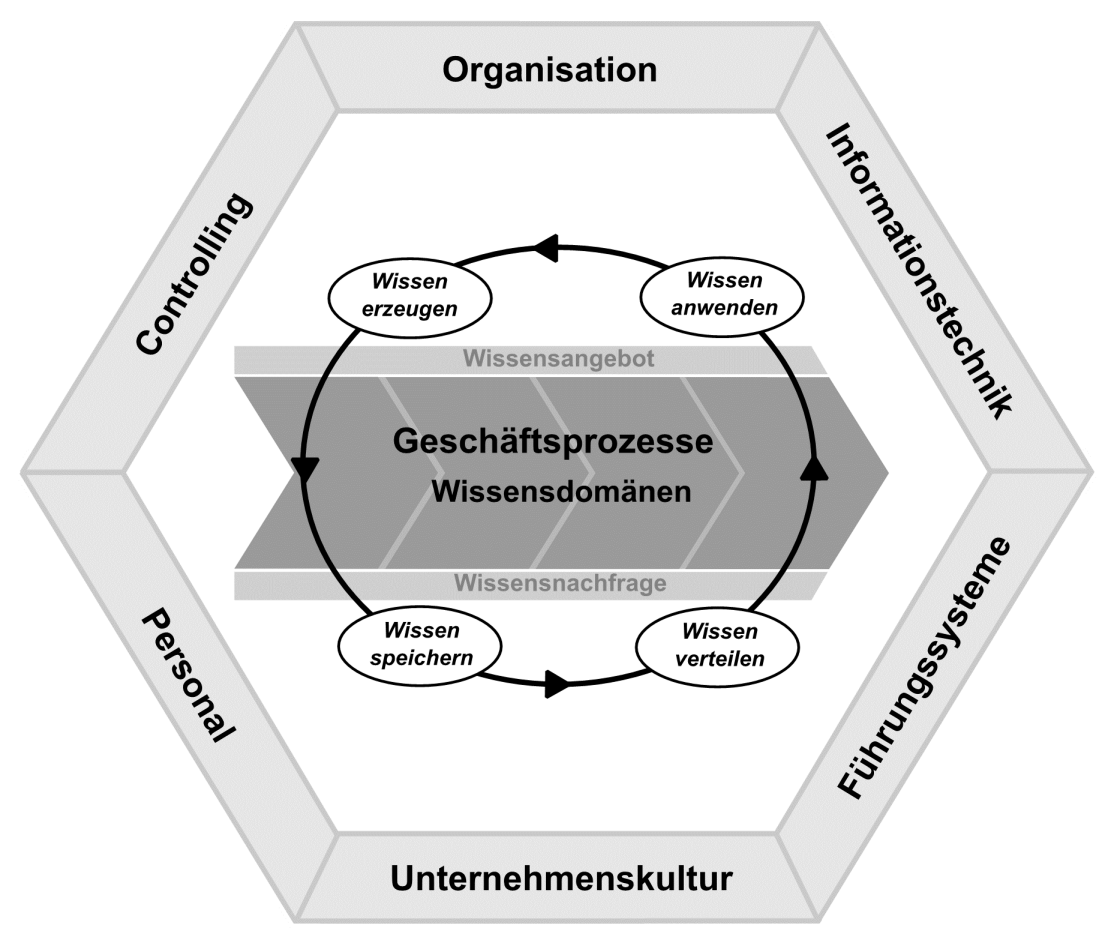

Abbildung 2-22: Wissensmanagement-Referenzmodell ${ }^{169}$

Wissensmanagementsysteme beschreiben das Vorgehen bei der Erzeugung und Verarbeitung von Wissen vorwiegend aus der Sicht der Managementebene in Unternehmen. Sie unterstützen den Anwender im Hinblick auf die Organisation und Verwaltung von unternehmensinternen Strukturen, die die Erhaltung und den Ausbau des Wissens im Unternehmen begünstigen. Das in einer Organisation vorhandene Wissen soll dazu möglichst vollständig erfasst und verarbeitet werden, um zu verhindern, dass relevante Informationen verloren gehen. Für die einzelnen Ansätze spielt es kaum eine Rolle, welche Art von Wissen verarbeitet wird. Im Gegensatz zu den genannten Ansätzen der Produktentwicklung, die sich mit der Aufarbeitung und Nutzung fertigungstechnologischen Wissens beschäftigen, fehlt es den Wissensmanagementsystemen an geeigneten Modellen und Beschreibungsstandards, um dieses Wissen für die Produktentwicklung nutzbar zu machen.

\footnotetext{
${ }^{168} \mathrm{Vgl}$. Mertins et al. (2016), S. $31 \mathrm{ff}$.

${ }^{169}$ Mertins et al. (2016), S. 32.
} 


\subsection{Fazit - Defizite hinsichtlich der systematischen Entwicklung fertigungsintegrierender Produktlösungen}

Die Entwicklung fertigungsintegrierender Produktlösungen stellt vor allem aufgrund der Vielfalt und Heterogenität fertigungstechnologischen Wissens eine große Herausforderung für den Produktentwickler dar (siehe Abschnitt 2.1). Vor allem textgebundene Hinweise, Regeln und Richtlinien sowie bildliche Darstellungen sind weitverbreitete Hilfsmittel, um dem Produktentwickler dieses Wissen zur Verfügung zu stellen (siehe Abschnitt 2.2). Deren kompakte Dokumentationsform reduziert das fertigungstechnologische Wissen auf die für den Produktentwickler wesentlichen Aussagen, die inn bei der Sicherstellung der Herstellbarkeit seiner Produkte unterstützen sollen. Auf diese Weise gehen jedoch auch Informationen verloren, die möglicherweise Ausgangspunkt für neuartige Produktlösungen sein können. Zudem können auf Basis dieser verkürzten Dokumentationsform fertigungstechnologischen Wissens kaum mehr Rückschlüsse über die Auswirkungen einer konstruktiven Festlegung auf den Fertigungsprozess getroffen werden.

Die im Rahmen des DfM/DfA vorgestellten Ansätze fokussieren vorwiegend ebenfalls das Ziel, die Herstellbarkeit der Produkte sicherzustellen. Das dazu notwendige fertigungstechnologische Wissen wird meist erst in späteren Entwicklungsphasen eingebracht, wo es häufig zu zeitraubenden Iterationen führt (siehe Abschnitt 2.3). Darüber hinaus existieren weitere Produktentwicklungsansätze, die zur gezielten Aufarbeitung und Nutzung fertigungstechnologischen Wissens geeignete Modelle verwenden. Die konsistente Modellierung von Produkten und Prozessen mittels Eigenschaften liefert die Grundlage, um fertigungstechnologisches Wissen strukturiert abzubilden und es den Eigenschaften des Produkts vergleichend gegenüberzustellen. Entsprechende Ansätze sind jedoch noch nicht soweit ausgearbeitet, dass fertigungstechnologisches Wissen umfassend für die Produktentwicklung nutzbar gemacht werden kann. Dazu fehlt es vor allem an der Möglichkeit, gezielt Verknüpfungen zwischen fertigungstechnologischem Wissen und dem Produkt herzustellen. Erst auf diese Weise können neuartige Lösungsmöglichkeiten für die Produktentwicklung systematisch erschlossen werden (siehe Abschnitt 2.4). Daneben gibt es Wissensmanagementsysteme, die in erster Linie als Hilfsmittel für die Managementebene von Unternehmen gedacht sind. Ihnen fehlt es vor allem an geeigneten Modellen und Beschreibungsstandards, um fertigungstechnologisches Wissen mit Blick auf die Produktentwicklung nutzbar zu machen (siehe Abschnitt 2.5). 


\section{Idee einer fertigungsintegrierenden Entwicklungsmethodik}

Das gezielte Einbeziehen fertigungstechnologischen Wissens in die Produktentwicklung eröffnet dem Entwickler nicht nur die Möglichkeit, die Herstellbarkeit seiner Produkte frühzeitig sicherzustellen, sondern auch, einen erkennbaren Mehrwert in Bezug auf die Produktfunktion sowie die Fertigungs- und Montageprozesse zu schaffen. Auch wenn der Gedanke, die Fertigung in die Lösungsfindung einzubeziehen, nicht neu ist, so zeigt sich dennoch, dass ein systematisches Produktentwicklungsvorgehen einer gezielten methodischen Unterstützung bedarf. Auf diese Weise kann fertigungstechnologisches Potenzial u. a. hinsichtlich Leichtbau, Produktlebensdauer, Bauteilsteifigkeit oder funktionaler Mehrwerte umfassend im Rahmen der Produktentwicklung ausgeschöpft werden.

Wie bereits in Kapitel 2 gezeigt, ist fertigungstechnologisches Wissen formal und inhaltlich sehr heterogen dokumentiert. So finden sich u. a. Graphen, Prinzipdarstellungen oder Formeln, um dieses Wissen zu repräsentieren. Zur Verwendung während der Produktentwicklung ist es notwendig, das fertigungstechnologische Wissen in einer einheitlichen Struktur standardisiert abzubilden, die eine grundsätzliche Übertragbarkeit der einzelnen Aussagen auf Modelle ermöglicht, die Produktentwickler zur Konkretisierung des Produkts verwenden. Die im Rahmen der modellbasierten Methodiken (siehe Abschnitt 2.4) verwendete Modellierung von Produkten und Prozessen mittels Eigenschaften bietet einen vielversprechenden Anknüpfungspunkt für die Standardisierung fertigungstechnologischen Wissens. Da fertigungstechnologisches Wissen mitunter sehr unterschiedliche Inhalte mit unterschiedlichem Konkretisierungsgrad adressiert, ist es notwendig, technische Prozesse ebenso wie technische Produkte auf unterschiedlichen Konkretisierungsebenen einheitlich zu modellieren. Es soll daher eine zur Produktmodellierung kompatible und in sich konsistente Prozessmodellwelt geschaffen werden, die über die Abbildung der Zustandsänderung hinausgeht und auch Wissen über die Verfahren und deren Verfahrensprinzipien umfassend abbildet. Auf diese Weise kann fertigungstechnologisches Wissen aus der Modellwelt der Fertigung in die Modellwelt der Produktentwicklung übertragen und modellübergreifend zu sogenannten fertigungsintegrierenden Lösungselementen verknüpft werden. Diese können genutzt werden, um systematisch fertigungsintegrierende Produktlösungen zu entwickeln (siehe Abbildung 3-1). 


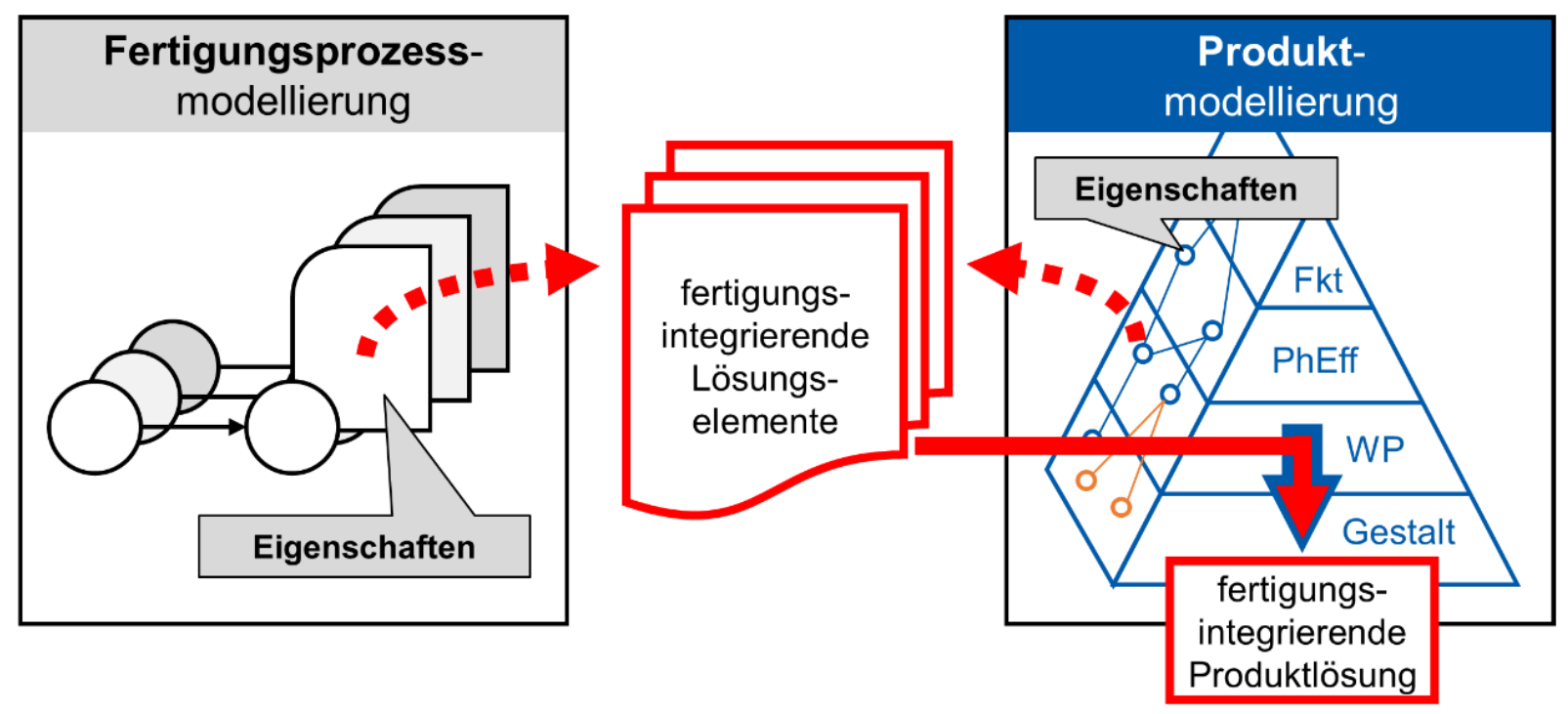

Abbildung 3-1: Schema zur modellübergreifenden Erarbeitung fertigungsintegrierender Produktlösungen

Neben dem Fehlen einheitlicher Modelle zur Abbildung fertigungstechnologischen Wissens zeigen die in Kapitel 2 vorgestellten Ansätze ebenfalls ein deutliches Defizit im Hinblick auf die methodische Unterstützung der Wissensbereitstellung und -verarbeitung im Rahmen der Produktentwicklung. Diese werden derzeit in weiten Bereichen nur unvollständig oder umständlich realisiert. Die vorgestellten Ansätze bedingen häufig aufwendige Iterationen, weisen eine nur unzureichende Operationalisierung auf oder sind aufgrund fehlender Modelle nur bedingt für die Aufarbeitung fertigungstechnologischen Wissens im Rahmen der Produktentwicklung geeignet. Erst modellbasierte Ansätze, wie die GPPE und die produktionsintegrierende Entwicklungsmethodik nach GRAMLICH ermöglichen ein frühzeitiges Antizipieren von Fertigungsprozessen im Produktentwicklungsprozess und deren modellbasierte Gegenüberstellung mit dem Produkt. Aufbauend auf deren Grundgedanken soll eine fertigungsintegrierende ${ }^{170}$ Entwicklungsmethodik erarbeitet werden, mittels derer die Aufbereitung fertigungstechnologischen Wissens ausgehend von der konsistenten Modellierung von Produkten und Prozessen unterstützt wird. Durch Verwendung der Methodik soll es möglich sein, fertigungsintegrierende Produktlösungen systematisch zu entwickeln.

170 Gegenüber dem Begriff „produktionsintegrierend“, der von GRAMLICH verwendet wird, betont der Begriff „fertigungsintegrierend“ einen stärkeren Bezug zum fertigungstechnologischen Wissen, das im Rahmen der hier vorgestellten Methodik erschlossen werden soll. 


\section{Standardisierung fertigungstechnologischen Wissens für die Produktentwicklung}

Die Standardisierung fertigungstechnologischen Wissens ist eine wesentliche Voraussetzung, um Wissen in einem übergeordneten Ansatz aufbereiten und weiterverarbeiten zu können. Den Ausgangspunkt bildet die eigenschaftsbasierte Modellierung der gewollten Zustandsänderung von Objekten. Durch Einbeziehung von Verfahren in die Prozessmodellierung wird der Grundstein für die Abbildung fertigungstechnologischen Wissens gelegt (siehe Abschnitt 4.1). Auf dieser Basis wird eine Modellwelt vorgestellt, die es ermöglicht, Prozesse und Verfahren in aufeinander aufbauenden Konkretisierungsebenen zu modellieren (siehe Abschnitt 4.2). Vor allem die Modellierung des Verfahrensprinzips eröffnet völlig neue Möglichkeiten für die standardisierte Abbildung fertigungstechnologischen Wissens. Es stellt gleichzeitig den fehlenden Baustein einer vollständig auf die Produktmodellwelt abgestimmten Prozess- und Verfahrensmodellierung dar (siehe Abschnitt 4.3), wodurch die modellübergreifende Verarbeitung von fertigungstechnologischem Wissen ermöglicht wird.

\subsection{Verfahren und fertigungstechnologisches Wissen}

Fertigungstechnologisches Wissen umfasst im Einklang mit der Definition aus Kapitel 1 allgemeingültige Aussagen über eine Fertigungstechnologie. Gemäß den Ausführungen in Abschnitt 2.1 beschreibt fertigungstechnologisches Wissen Sachverhalte, die sich je nach Verfahren deutlich unterscheiden. Die Benennung und Gliederung bekannter Fertigungsverfahren, wie sie z. B. in der DIN 8580 ${ }^{171}$, bei FRITZ UND SCHULZE ${ }^{172}$ oder bei WESTKÄMPER UND WARNECKE ${ }^{173}$ zu finden sind, liefert somit eine erste Übersicht über das existierende fertigungstechnologische Wissen, gegliedert nach Verfahren. Durch Kenntnis des fertigungstechnologischen Wissens sind die Stellhebel bekannt, mittels derer der Fertigungsprozess konkret beeinflusst werden kann.

Verfahren erklären, auf welche Art und Weise die Zustandsänderung eines Operanden, d. h. das Erreichen charakteristischer verfahrensinduzierter Eigenschaf-

\footnotetext{
171 Vgl. DIN 8580 (2003).

172 Vgl. Fritz, Schulze (2010).

${ }^{173}$ Vgl. Westkämper, Warnecke (2010).
} 
ten realisiert wird. Dies umfasst auch das Verfahrensprinzip, das das Zusammenwirken der am technischen Prozess beteiligten Wirkelemente und Wirkgrößen beschreibt und damit neben dem Operanden auch das Arbeitsmittel des Prozesses in die Betrachtungen einbezieht. Das Arbeitsmittel erfährt in dieser Betrachtung selbst jedoch keine gewollte Zustandsänderung. ${ }^{174}$ Fertigungsverfahren werden durch fertigungstechnologisches Wissen u. a. über die Wirkungsabläufe, Wirkzusammenhänge, Arbeitsmittel, Operanden oder Wirkgrößen charakterisiert, das für die Realisierung eines technischen Prozesses notwendig ist. Dieses fertigungstechnologische Wissen beschreibt die Einflussmöglichkeiten auf die Erzeugung und Änderung der verfahrensinduzierten Eigenschaften. Die Aussage „Verfahren realisieren Prozesse" 175 bedeutet somit, dass mit der Wahl des Verfahrens das entsprechende fertigungstechnologische Wissen zur Verfügung steht, das genutzt werden kann, um den technischen Prozess weiter zu konkretisieren.

Im Hinblick auf den Produktentwicklungskontext muss fertigungstechnologisches Wissen vor allem hinsichtlich seiner Relevanz unterschieden werden, die es für den Produktentwickler hat. Abbildung 4-1 a) zeigt ein Beispiel für fertigungstechnologisches Wissen, das den Zusammenhang zwischen der Axialkraft $F_{\text {axial }}$ und dem Innendruck $p_{\mathrm{i}}$ für das Fertigungsverfahren Innenhochdruckumformen erläutert. Die angegebenen Prozessfenster lassen jedoch keine direkten Aussagen über die erreichbare Gestalt des gefertigten Bauteils zu. Für die Lösungsfindung im Rahmen der Produktentwicklung hat die Kenntnis möglicher Prozessfenster des Innenhochdruckumformens daher keine erkennbare Relevanz, sofern nur rein technische Gesichtspunkte betrachtet werden. Wirtschaftliche Gesichtspunkte, wie z. B. Kosten, die sich aus den Fertigungsprozessen ergeben, werden im Rahmen dieser Arbeit nicht näher betrachtet.

Wissen, das für die Produktentwicklung relevant ist, muss also immer auch das Produkt umfassen. Produkt-Wissen betrifft so z. B. Zusammenhänge, die u. A. die Produktfunktion näher erläutern. Abbildung 4-1 c) zeigt hierzu drei Federkennlinien, die den Zusammenhang zwischen der aufgebrachten Kraft $\Delta F$ und dem da-

\footnotetext{
174 Vgl. Gramlich (2013), S. 93.

${ }^{175}$ Diese Aussage bezieht sich nicht nur auf Fertigungsprozesse, sondern auch auf alle anderen technischen Prozesse des Produktlebenslaufs. Dies ist möglich, da alle technischen Prozesse des Produktlebenslaufs entweder wie Fertigungsprozesse modelliert werden oder Einsatzprozesse sind, die selbst wiederum als Fertigungsprozess verstanden werden können, in denen das Produkt als Arbeitsmittel fungiert.
} 
raus resultierenden Federweg $\Delta s$ qualitativ darstellen. Dies führt zu der Schlussfolgerung, dass auch nur solches fertigungstechnologische Wissen für die Produktentwicklung relevant ist, das Objekte oder technische Produkte adressiert. Abbildung 4-1 b) zeigt den Einfluss der kleinsten Innenradien eines Bauteils $r_{\min }$ (bezogen auf die Ausgangswanddicke $s_{0}$ ) auf den Kalibrierdruck $p_{\text {i max }}$ (bezogen auf die Umformfestigkeit $k_{\mathrm{f}}$ ) am Beispiel des Innenhochdruckumformens. Auf diese Weise können Limitierungen des Verfahrens direkt auf den Operanden bezogen werden. Für den Produktentwickler hat dieser Zusammenhang eine hohe Relevanz, da er anhand dessen seine Produktgestalt mit Bezug auf die verwendeten Halbzeuge anpassen kann. Dieses fertigungstechnologische Wissen wird als fertigungstechnologisches Produkt-Wissen bezeichnet.

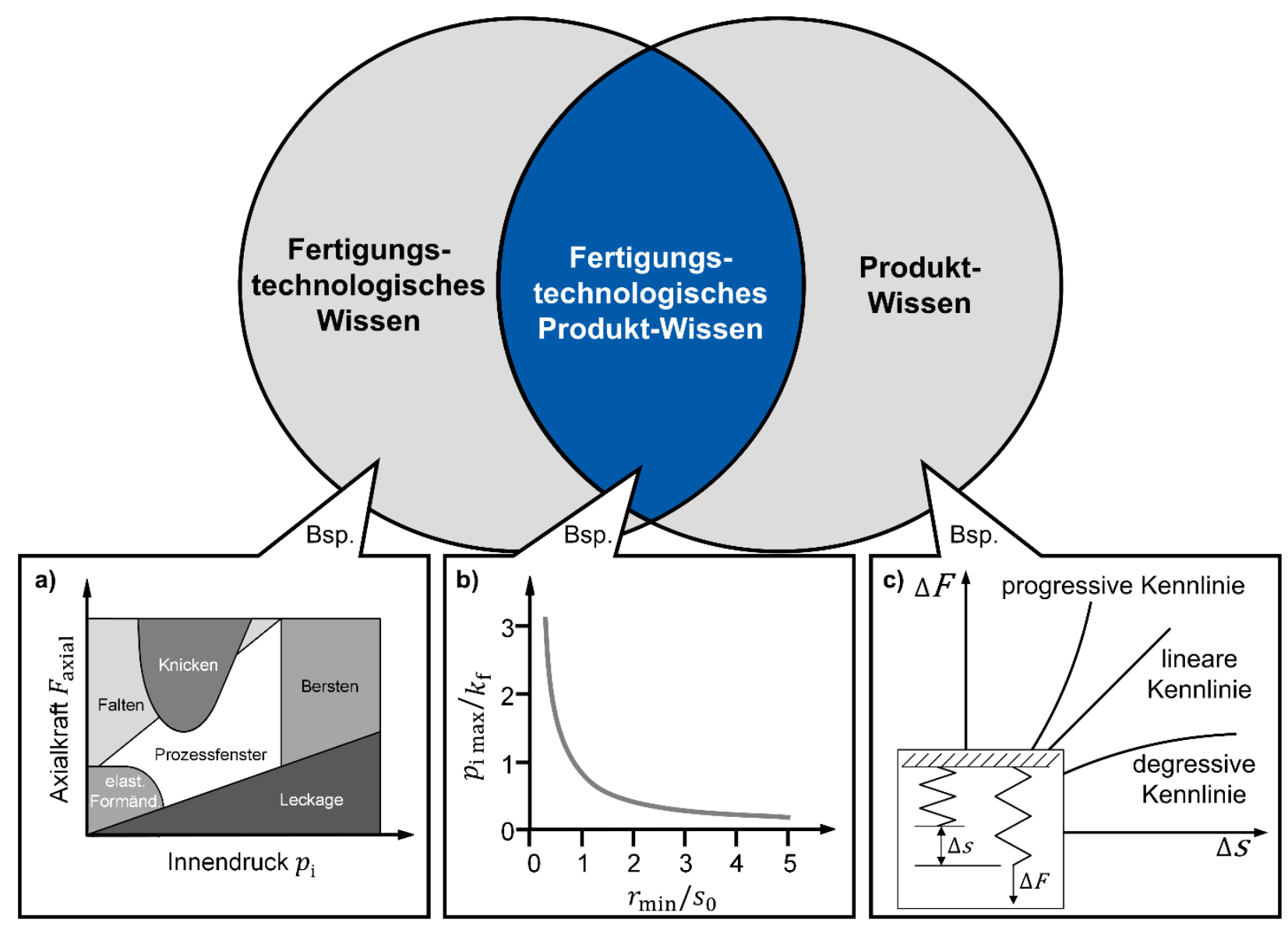

Abbildung 4-1: Fertigungstechnologisches Wissen, fertigungstechnologisches ProduktWissen und Produkt-Wissen anhand ausgewählter Beispiele; a) Prozessfenster beim Innenhochdruckumformen ${ }^{176}$ b) Auf die Umformfestigkeit bezogener Kalibrierdruck in Abhängigkeit von der Bauteilgeometrie ${ }^{177}$; c) Federkennlinien

\footnotetext{
${ }^{176}$ Bahn et al. (2007), S. 80 in Anlehnung an VDI 3146-1 (1999).
}

177 Bahn et al. (2007), S. 81 in Anlehnung an Hartl (1999). 
Fertigungstechnologisches Produkt-Wissen muss über entsprechende einheitliche Modelle, die das Verfahren mit all seinen Aspekten beschreiben, abgebildet werden, um die dokumentierten Zusammenhänge für die Produktentwicklung nutzbar zu machen. Dies erfordert jedoch die Verwendung eines einheitlichen Beschreibungsstandards für fertigungstechnologisches Wissen. Einen Anknüpfungspunkt bietet die Beschreibung mittels Eigenschaften. Aussagen zu Produkten werden im Kontext der Produktfunktion mittels Eigenschaften beschreibbar, die zueinander in Relation stehen. Fertigungstechnologisches Produkt-Wissen umfasst Aussagen zu den Produkten im Rahmen des betrachteten Verfahrens. Dementsprechend können diese Aussagen ebenfalls mittels Eigenschaften und deren Relationen beschrieben werden. ${ }^{178}$ Auf diese Weise wird fertigungstechnologisches Produkt-Wissen standardisiert beschreibbar, sodass es im Kontext der Produktentwicklung verwendet werden kann. Bislang existiert jedoch noch kein einheitliches Modell, das diesen Beschreibungsstandard zur Abbildung fertigungstechnologischen Produkt-Wissens unter der Angabe von Eigenschaftsrelationen nutzt.

\subsection{Konkretisierungsebenen der Prozessmodellierung}

\subsubsection{Modellierung von Prozessen auf Basis der Zustandsänderung}

Die umfassende und gleichzeitig einheitliche Abbildung fertigungstechnologischen Produkt-Wissens ist nur mittels geeigneter Prozessmodelle möglich. Im Hinblick auf die Aufbereitung des Wissens für die Produktentwicklung sind vor allem solche Prozessmodelle von Interesse, deren Beschreibung auf Eigenschaften basiert. Die Abbildung fertigungstechnologischen Produkt-Wissens erfordert insbesondere die Abbildung von Eigenschaftsrelationen. Die Modellierung der Zustandsänderung stellt die abstrakteste Form der Prozessmodellierung dar. Dieser Konkretisierungsgrad der Modellierung eignet sich insbesondere für die Darstellung von Prozessstrukturen als Abfolge bestimmter Zustände des Operanden des technischen Prozesses. Die Zustandsänderung kann dabei nicht ohne Berücksichtigung der zeitlichen Abfolge der einzelnen Zustände des Operanden beschrieben werden.

\section{Modellierung von einzelnen Prozessen}

Prozesse im Sinne einer Zustandsänderung werden durch Verfahren realisiert. Das gewählte Verfahren gibt somit vor, welche Zustände erreicht werden können,

${ }^{178}$ Vgl. Roos et al. (2014), S. 1857. 
bzw. auf Basis welcher Anfangszustände ein bestimmter Endzustand erreicht werden kann. Die Angabe des Verfahrens für die Modellierung der Zustandsänderung ist unabdingbar, da es den spezifischen technischen Prozess ohne geeignetes Verfahren nicht gäbe (siehe Abbildung 4-2).

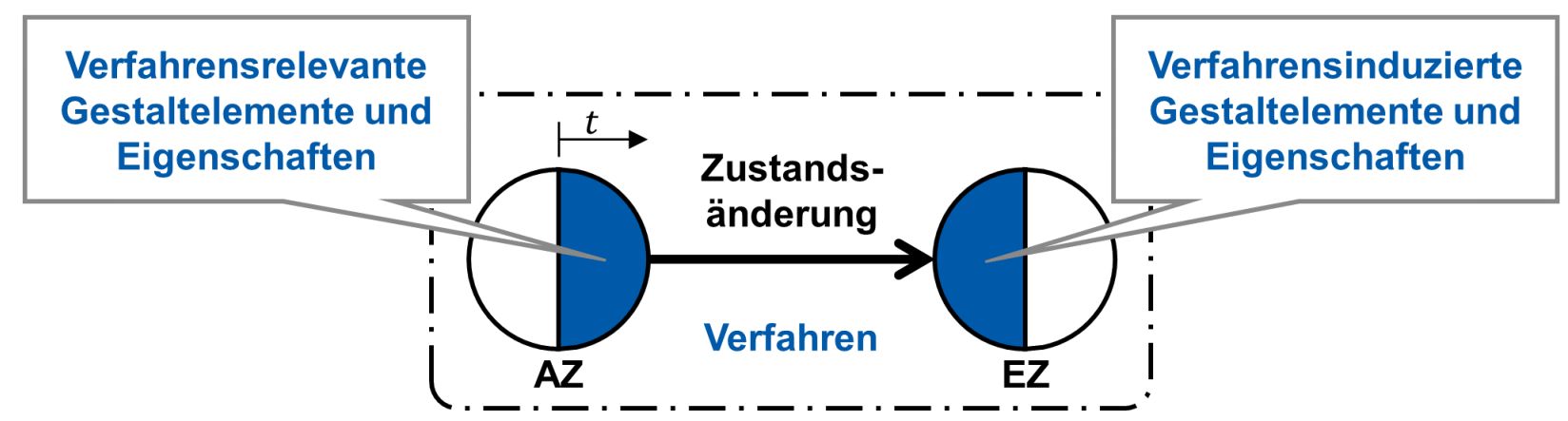

Abbildung 4-2: Modellierung der Zustandsänderung für ein bestimmtes Verfahren

Die Zustände des Operanden werden über Gestaltelemente und deren Eigenschaften beschrieben. Das gewählte Verfahren gibt vor, welche Gestaltelemente und Eigenschaften im Endzustand erreichbar sind (verfahrensinduzierte Gestaltelemente ${ }^{179}$ ) und welche im Anfangszustand benötigt werden (verfahrensrelevante Gestaltelemente). Die verfahrensrelevanten Gestaltelemente geben an, welche geometrischen und werkstofflichen Eigenschaften des Halbzeugs notwendig sind, um den Prozess durchführen zu können. Am Beispiel einer Zustandsänderung, die auf Basis des Spaltprofilierens durchgeführt wird (siehe Abbildung 4-3) zeigt sich zudem, dass es für die Modellierung unerheblich ist, alle Eigenschaften des Operanden im Anfangszustand zu kennen. Für die grundsätzliche Durchführbarkeit der Zustandsänderung sind nur Eigenschaften zu erfassen, die die relevanten Gestaltelemente betreffen (blau markierte Teile des Anfangszustands in Abbildung 4-3). Für das Spaltprofilieren sind das vor allem die Eigenschaften Blechdicke, Blechbreite und Werkstoff, die den Anfangszustand des Blechhalbzeugs beschreiben.

${ }^{179}$ Vgl. Gramlich (2013), S. $90 \mathrm{ff}$. 


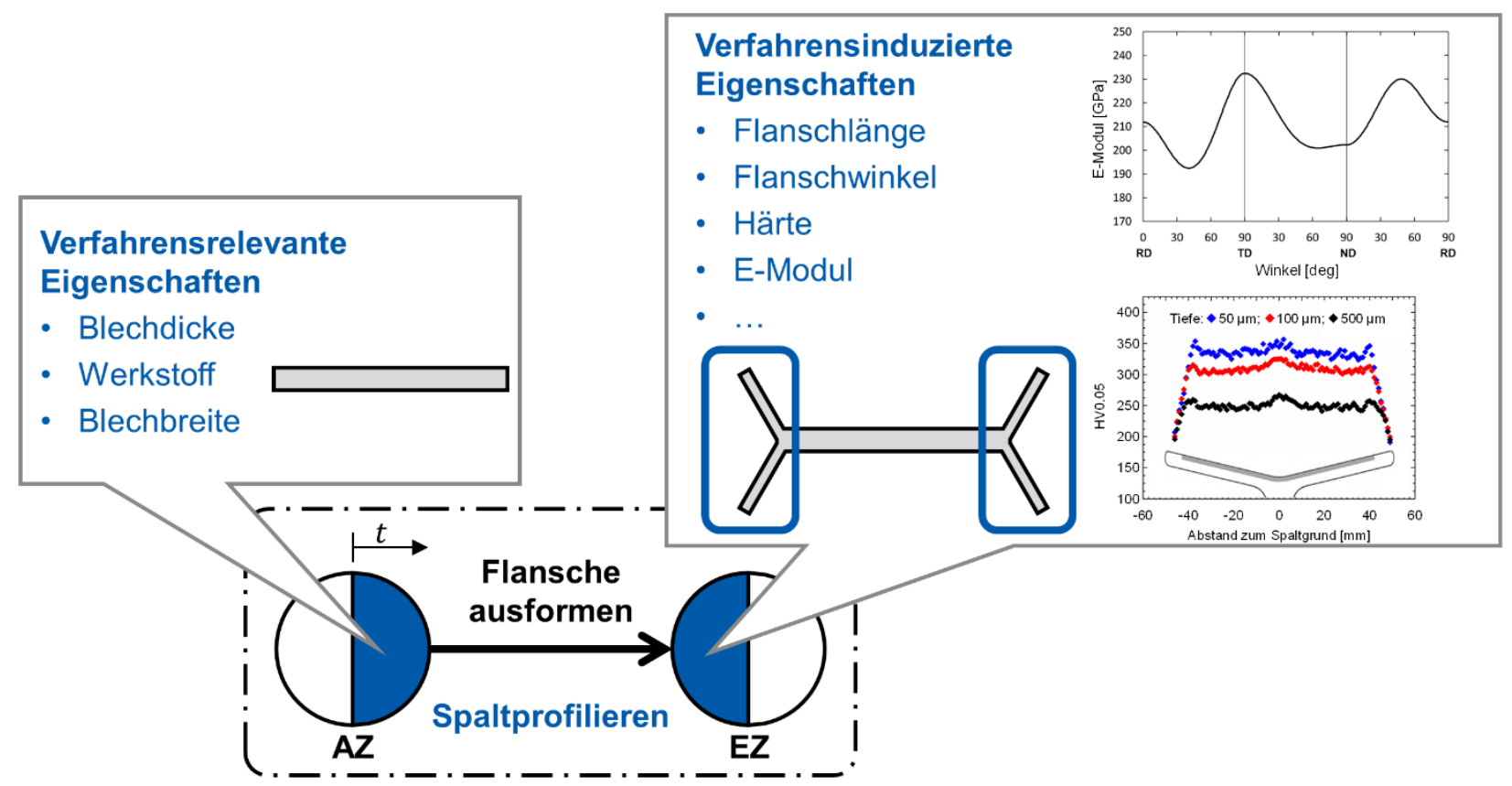

Abbildung 4-3: Modellierung des Ausformens von Flanschen mittels Spaltprofilieren

Im Endzustand liegen durch die Zustandsänderung erzeugte Gestaltelemente die spaltprofilierten Flansche - an den Bandkanten vor, die u. a. durch eine bestimmte Flanschlänge, einen Flanschwinkel, eine erhöhte Härte sowie einen anisotropen E-Modul charakterisiert sind. ${ }^{180}$ Eine detailliertere Angabe der Eigenschaften der spaltprofilierten Gestaltelemente kann z. B. in Form grafischer Verläufe gegeben werden (siehe Abbildung 4-3). Auch für den Endzustand gilt, dass die verfahrensinduzierten Gestaltelemente und deren Eigenschaften nur einen Teil des Endzustands des Operanden im technischen Prozess abbilden. Der Operand ist in diesem Zustand ebenso durch Eigenschaften beschreibbar, die nicht durch das Verfahren in der vorausgegangenen Zustandsänderung erzeugt wurden. So weist das spaltprofilierte Bauteil auch Gestaltelemente und Eigenschaften auf, die auf das verwendete Halbzeug zurückzuführen sind und nicht durch das Verfahren erzeugt bzw. verändert worden sind. Darunter fallen beispielsweise auch die Ausprägungen der Festigkeit und der Härte des Stegbereichs, die auf dem Niveau des Ausgangsmaterials liegen. ${ }^{181}$

Die Zustände des Operanden werden demnach zwar durch eine Vielzahl weiterer Eigenschaften beschrieben (siehe weiße Halbkreise in Abbildung 4-3). Zur Charakterisierung der Zustandsänderung im Kontext eines bestimmten Verfahrens genügen jedoch die angegebenen Eigenschaften, die sowohl die zur Durchführung

${ }^{180}$ Vgl. Neuwirth et al. (2017), S. 31 ff.; Ahmels et al. (2017), S. 99 ff.

${ }^{181}$ Vgl. Jöckel (2005), S. 84 ff. 
des Verfahrens unbedingt erforderlichen Eigenschaften (verfahrensrelevante Eigenschaften) sowie die Eigenschaften der durch dieses Verfahren charakteristisch erzeugten Gestaltelemente (verfahrensinduzierte Eigenschaften) beschreiben.

Am Beispiel des Innenhochdruckumformens von Rohren (siehe Abbildung 4-4) werden im Anfangszustand Bauteile benötigt, die einen geschlossenen Rohrquerschnitt aufweisen. Entsprechende verfahrensrelevante Eigenschaften sind der Rohrdurchmesser, die Wanddicke, die Rohrlänge und der Werkstoff. Durch das Innenhochdruckumformen wird eine Zustandsänderung realisiert, die zur Ausbildung von Gestaltelementen wie z. B. Domen führt. Diese sind dementsprechend durch die Domhöhe, den Domdurchmesser, dessen Wanddicke und den Winkel, unter dem der Dom zum Rohr steht, beschrieben.

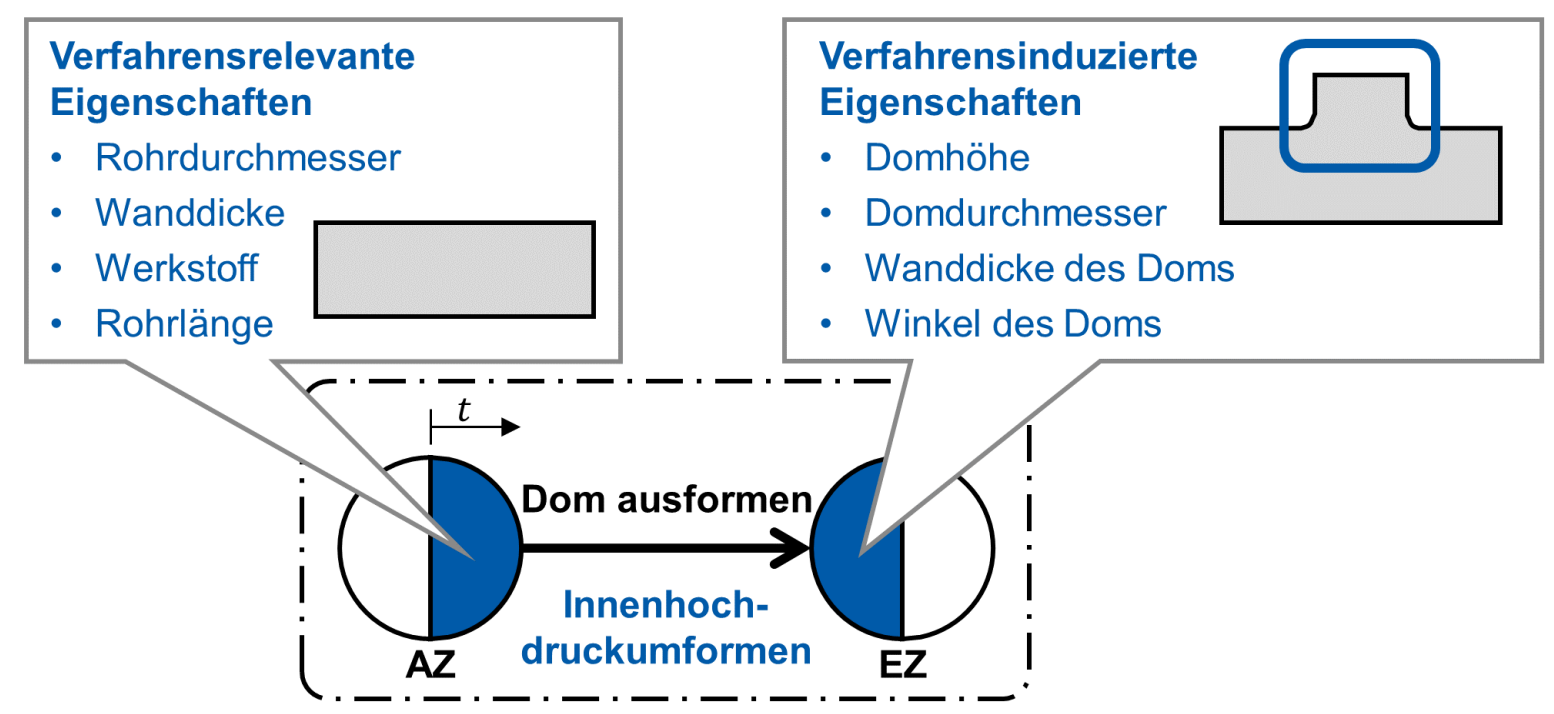

Abbildung 4-4: Modellierung des Ausformens eines Doms mittels Innenhochdruckumformen

\section{Modellierung von Prozessketten}

Auch Prozessstrukturen bzw. Prozessketten ${ }^{182}$ können wie die Zustandsänderung einzelner Prozesse mittels Eigenschaften und zugrunde liegende Verfahren beschrieben werden. Für Prozessketten gilt, dass nur die verfahrensinduzierten Eigenschaften sowie die verfahrensrelevanten Eigenschaften der kompletten Prozesskette abgebildet werden, da nur diese charakteristisch für die gewählte Prozesskette sind. Dabei beinhalten die verfahrensinduzierten Eigenschaften im End-

182 Als Prozessketten werden in dieser Arbeit alle Prozessstrukturen bezeichnet, die eine lineare nicht verzweigte Struktur aus Zustandsänderungen aufweisen. 
zustand nicht nur verfahrensinduzierte Eigenschaften des letzten Verfahrens, sondern auch verfahrensinduzierte Eigenschaften aller vorgelagerten Verfahren der Prozesskette, die durch das letzte Verfahren nicht weiter geändert wurden. So ändert das Verfahren Ablängen nur die Bauteillänge, z. B. von Rohren. Die RohrForm ist keine verfahrensinduzierte Eigenschaft dieses Prozesses. Für ein nachfolgendes Innenhochdruckumformen des abgelängten Rohres ist die Rohr-Form hingegen eine notwendige Information, die als verfahrensrelevante Eigenschaft für die Durchführung der Umformung geklärt sein muss. Es ist daher unabdingbar auch die verfahrensinduzierten Eigenschaften aller dem Ablängen vorgelagerten Prozesse zu kennen, um Aussagen über die Durchführbarkeit eines nachgelagerten Prozesses, wie dem Innenhochdruckumformen, treffen zu können. Aus diesem Grund wird für eine Prozesskette immer die Summe der verfahrensinduzierten Eigenschaften der zugrunde liegenden Verfahrenskette ${ }^{183}$ angegeben.

Für die Modellierung von Prozessketten und einzelner Prozesse gilt zudem ein gemeinsamer Grundsatz: Zwischenzustände müssen nicht modelliert werden. Es werden daher nicht zwangsweise Eigenschaften zu den Zwischenzuständen angegeben. Lediglich der Anfangs- und Endzustand werden eindeutig und charakteristisch für das gewählte Verfahren bzw. die gewählte Verfahrenskette durch verfahrensrelevante und verfahrensinduzierte Eigenschaften beschrieben (siehe Abbildung 4-5).

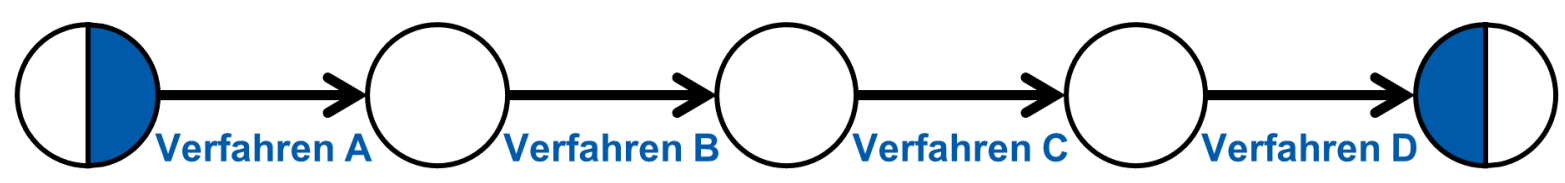

Abbildung 4-5: Modellierung einer Prozesskette

\subsubsection{Modellierung von Verfahren auf Basis physikalischer Effekte}

SAUER benennt physikalische, chemische oder biologische Wirkungsabläufe als wesentlichen Bestandteil von Verfahren. ${ }^{184}$ Die einzelnen Wirkungsabläufe beruhen auf physikalischen, chemischen oder biologischen Gesetzmäßigkeiten, ohne die ein Verfahren nicht realisierbar wäre. Zur weiteren Konkretisierung der Zu-

\footnotetext{
183 Die definierte Abfolge von Verfahren wird als Verfahrenskette bezeichnet. Die Zustände des Operanden sind bei dieser Betrachtung nicht von Bedeutung.

${ }^{184}$ Vgl. Sauer (2006), S. 74.
} 
standsänderung ist es daher zweckmäßig, physikalische, chemische und biologische Gesetzmäßigkeiten ${ }^{185}$ näher zu betrachten und diese in einem entsprechenden Modell abzubilden. Sie erläutern die Art und Weise wie die eigentliche Zustandsänderung initiiert wird. Insbesondere sind hier diejenigen Effekte von Interesse, die auf der Transformation von Stoffen beruhen. Tabellarische Übersichten physikalischer Effekte, wie sie z. B. bei KoLLER ${ }^{186}$ zu finden sind, können dabei gezielt zur Unterstützung der Lösungsfindung eingesetzt werden.

Abbildung 4-6 zeigt das Verfahren Spaltprofilieren, das durch physikalische Effekte näher erläutert wird. Durch Aufbringen einer ausreichend hohen Kraft $F$ bilden sich Spannungen in der Umformzone aus. Das Material beginnt zu fließen, sobald die vorhandenen Spannungen oberhalb der Fließgrenze $R_{\mathrm{e}}$ liegen. Der physikalische Effekt beschreibt, dass es infolge des Aufbringens einer ausreichend hohen Kraft zu einer plastischen Verformung des Operanden kommt. Dabei haben vor allem die Fläche $A$ und die Fließspannung $R_{\mathrm{e}}$ - beide Eigenschaften des Operanden im Anfangszustand - Einfluss auf die aufzubringenden Kräfte. Durch Abstützung der Umformzone wird ein hydrostatischer Druckspannungszustand erreicht, der zu einem gerichteten Fließen des verdrängten Materials führt. Dies hat zur Folge, dass sich ein UFG Gefüge ausbildet. ${ }^{187}$

$$
\sigma=\frac{F}{A}>R_{\mathrm{e}}
$$

Plastische Verformung: Überschreiten der Fließspannung $R_{\mathrm{e}}$ bei Kraft $F$ auf ein Fläche $A$ führt zu plastischer Verformung
Gerichtetes Fließen: Allseitige Abstützung („Kanalisierung“) eines Werkstücks während der Umformung führt unter Vorhandensein eines hydrostatischen Druckspannungszustands zur gerichteten Ausformung von Gestaltelementen mit speziellen Gefügeeigenschaften

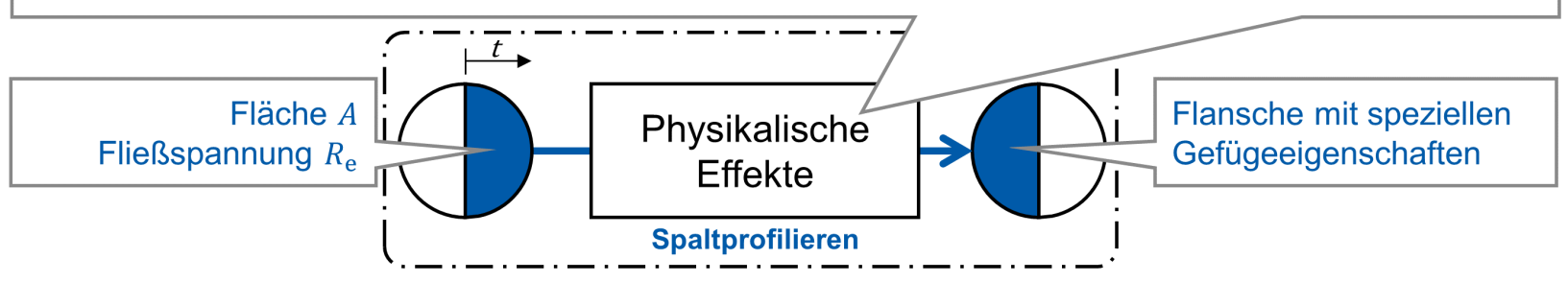

Abbildung 4-6: Physikalische Effekte für das Verfahren Spaltprofilieren

Das Innenhochdruckumformen ist in erster Linie durch das plastische Fließen eines geschlossenen Rohres unter Innendruck charakterisiert (siehe Abbildung 4-7).

\footnotetext{
185 Im Folgenden wird der Begriff der physikalischen Gesetzmäßigkeiten stellvertretend für physikalische, biologische und chemische Gesetzmäßigkeiten verwendet.

${ }^{186}$ Vgl. Koller (1994), S. 547 ff.

${ }^{187}$ Vgl. Neuwirth et al. (2017), S. $40 \mathrm{ff}$.
} 
Voraussetzung für die Durchführung der Zustandsänderung ist ein Halbzeug mit geschlossenem Querschnitt (Durchmesser $d_{\mathrm{m}}$ ). Durch Aufbringen eines Innendrucks $p$ bildet sich gemäß der Kesselformel eine Tangentialspannung $\sigma_{\mathrm{t}}$ im Rohr aus, die bei Überschreiten der Streckgrenze $R_{\mathrm{e}}$ zum Fließen des Materials führt. Das Rohr weitet sich auf diese Weise auf.

Plastische Verformung unter Innendruck: Bei ausreichend hohem Druck im geschlossenen Rohr mit Durchmesser $d_{\mathrm{m}}$ wird die Fließspannung $R_{\mathrm{e}}$ in der Rohrwand (Tangentialspannung) überschritten und der Werkstoff $\sigma_{\mathrm{t}}=\frac{p \cdot d_{\mathrm{m}}}{2 \cdot s}>R_{\mathrm{e}}$ beginnt zu fließen, sodass sich das Rohr aufweitet

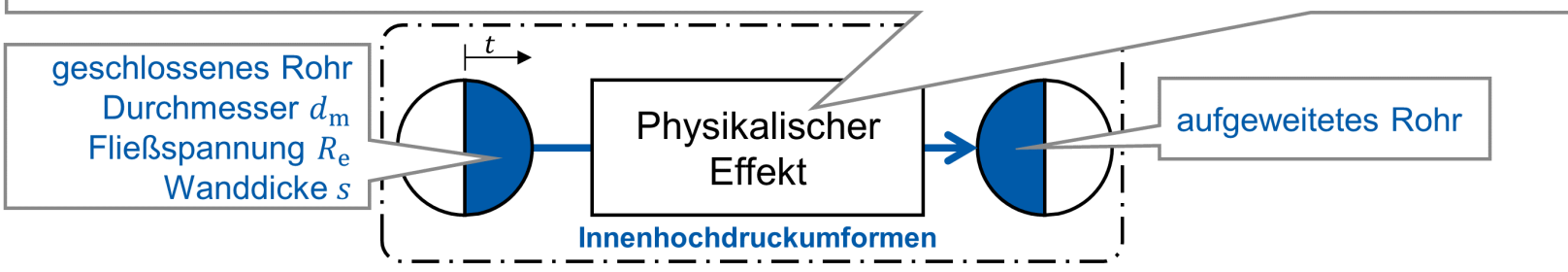

Abbildung 4-7: Physikalischer Effekt für das Verfahren Innenhochdruckumformen

Durch die Modellierung technischer Prozesse unter Einbeziehung physikalischer Effekte kann bereits fertigungstechnologisches Produkt-Wissen abgebildet werden. Das Modell erlaubt es, Eigenschaften und deren Vernetzungen abzubilden, die hier meist als Formeln vorliegen. Die allgemeingültigen physikalischen Gesetzmäßigkeiten sind jedoch nicht an konkrete technische Objekte gebunden. Die Modellierung gibt somit keine Auskunft darüber, in welcher Weise die am technischen Prozess beteiligten Objekte miteinander interagieren.

\subsubsection{Modellierung von Verfahren auf Basis des Verfahrensprinzips}

Durch die Modellierung von Verfahren unter Berücksichtigung des Verfahrensprinzips soll fertigungstechnologisches Produkt-Wissen möglichst umfassend abgebildet werden. Durch die eigenschaftsbasierte Modellierung der am Verfahrensprinzip beteiligten Wirkelemente sowie derer Wirkzusammenhänge kann letztlich das Zusammenspiel der am technischen Prozess beteiligten Objekte näher beschrieben werden. Das Modell schließt damit eine Lücke in der Prozessmodellwelt, indem Wirkzusammenhänge in ähnlich formaler Weise abgebildet werden, wie dies im Kontext der Produktmodellierung der Fall ist. Ausgehend von der grundlegenden Idee des Modells wird ein eigenschaftsbasiertes Modell vorgestellt, das auf der Abbildung von Verfahrensprinzipien beruht. Anhand der Beispiele Innenhochdruckumformen und Spaltprofilieren wird das Modell erläutert. 


\section{Definition und Idee des Verfahrensprinzips}

Gemäß Abschnitt 2.4.2 beschreibt das Verfahrensprinzip eines Verfahrens den Wirkzusammenhang zwischen Wirkelementen und Wirkgrößen, die an der Realisierung der Zustandsänderung des Operanden beteiligt sind. Zur Abbildung fertigungstechnologischen Produkt-Wissens ist es unabdingbar, diese Wirkzusammenhänge in einem konsistenten Modell abzubilden. Neben der Beschreibung durch Eigenschaften sind dazu auch Eigenschaftsrelationen notwendig.

Abbildung 4-8 gibt die Verfahrensprinzipien für das Spaltprofilieren und das Innenhochdruckumformen wieder. Die jeweilige Darstellung stellt das Verfahrensprinzip in seiner abstraktesten Form dar, die alle Modellelemente abbildet, deren Zusammenhänge jedoch nur rudimentär wiedergibt. Es wird darin veranschaulicht, welche Elemente miteinander interagieren, worauf Wirkgrößen einwirken und worin die Zustandsänderung besteht. Verfahrensprinzipien beruhen auf physikalischen Gesetzmäßigkeiten (siehe Abschnitt 4.2.2) bzw. konkretisieren diese, indem sie alle am Verfahren beteiligten Wirkelemente und Wirkgrößen in einen Wirkzusammenhang bringen. Durch die Zuordnung physikalischer Größen zu bestimmten Wirkelementen bzw. Wirkgrößen erklärt sich der Wirkzusammenhang.
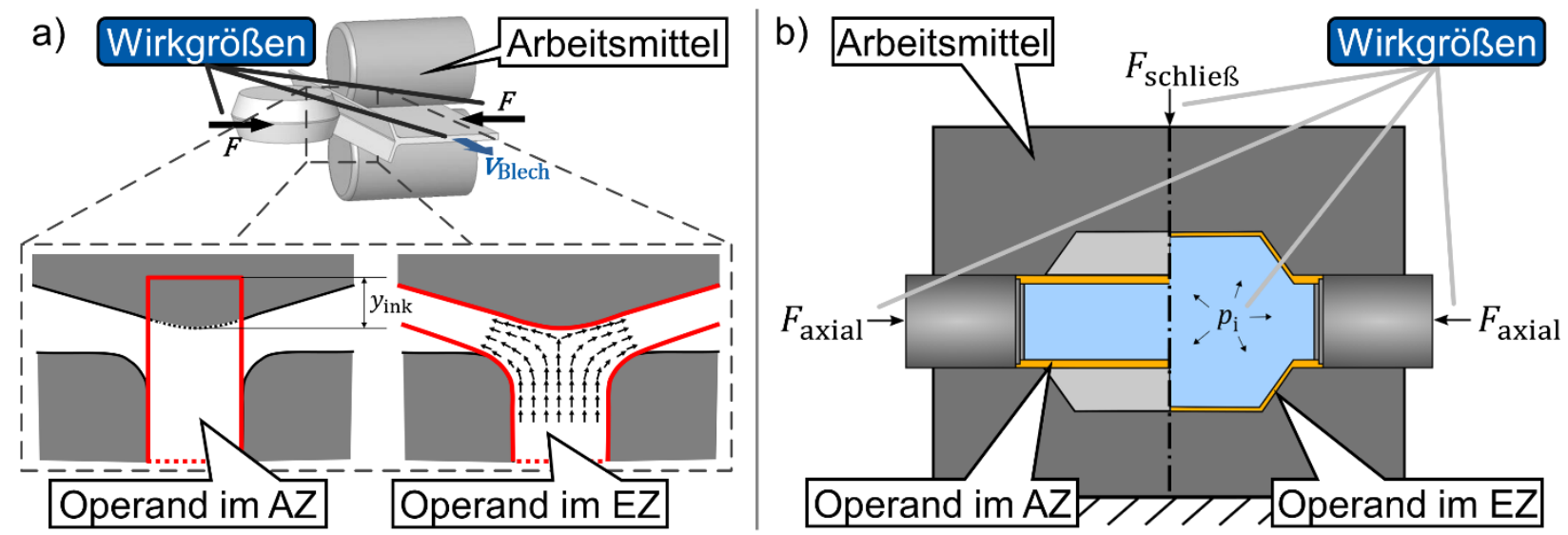

Abbildung 4-8: Verfahrensprinzipien und deren Elemente am Beispiel a) Spaltprofilieren ${ }^{188}$ und b) Innenhochdruckumformen ${ }^{189}$

Verfahrensprinzipien können durch das fertigungstechnologische Wissen beschrieben werden, auf dem die Wirkzusammenhänge beruhen. Die Idee einer Modellierung des Verfahrensprinzips zur Abbildung fertigungstechnologischen Produkt-Wissens beruht daher auf der Abbildung von Wirkzusammenhängen unter

\footnotetext{
${ }^{188}$ In Anlehnung an Gramlich (2013), S. 88.

189 In Anlehnung an Noack, Sterzing (2007), S. 15.
} 
Berücksichtigung der am Prozess beteiligten Produkte und ihrer Wirk- und Gestaltelemente (siehe Abbildung 4-9).

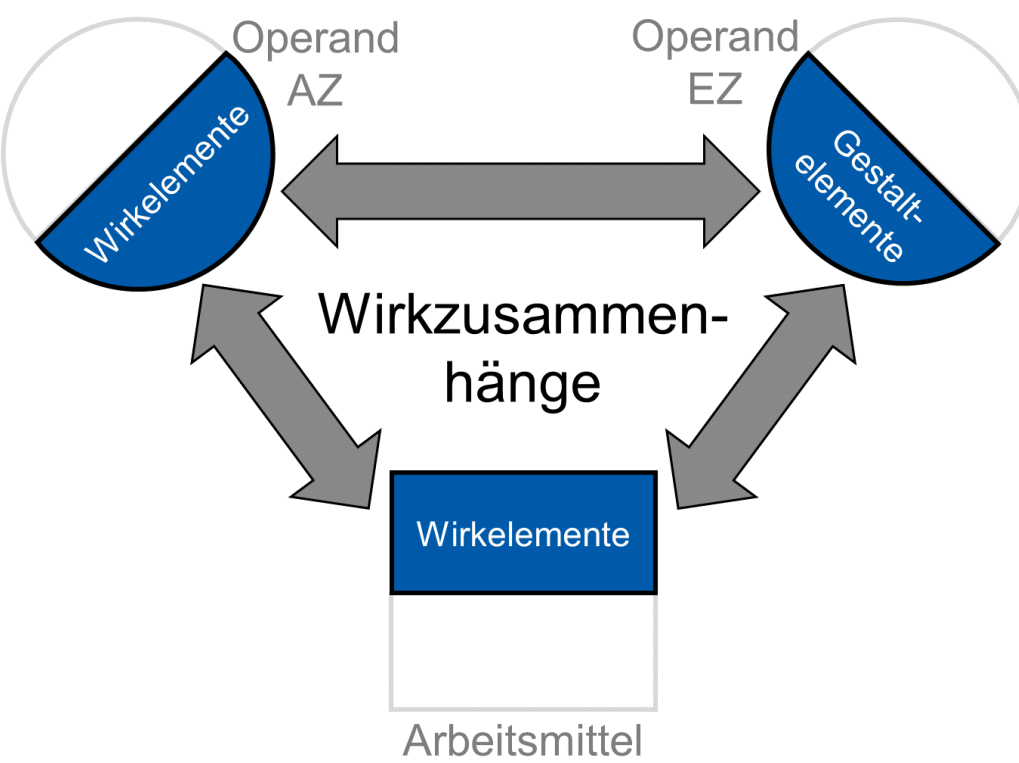

Abbildung 4-9: Kerngedanke des Verfahrensprinzips

\section{Modellierung des Verfahrensprinzips mittels Eigenschaften und}

\section{Eigenschaftsrelationen}

Die entscheidenden Wirkzusammenhänge, die durch entsprechendes fertigungstechnologisches Produkt-Wissen wiedergeben werden, können sehr unterschiedlich dokumentiert sein. Es ist deshalb notwendig, ein Modell zu schaffen, das die vorhandenen Wirkzusammenhänge in einer einheitlichen Struktur abbildet - das sogenannte Verfahrensprinzipmodell (VP-Modell) (siehe Abbildung 4-10).

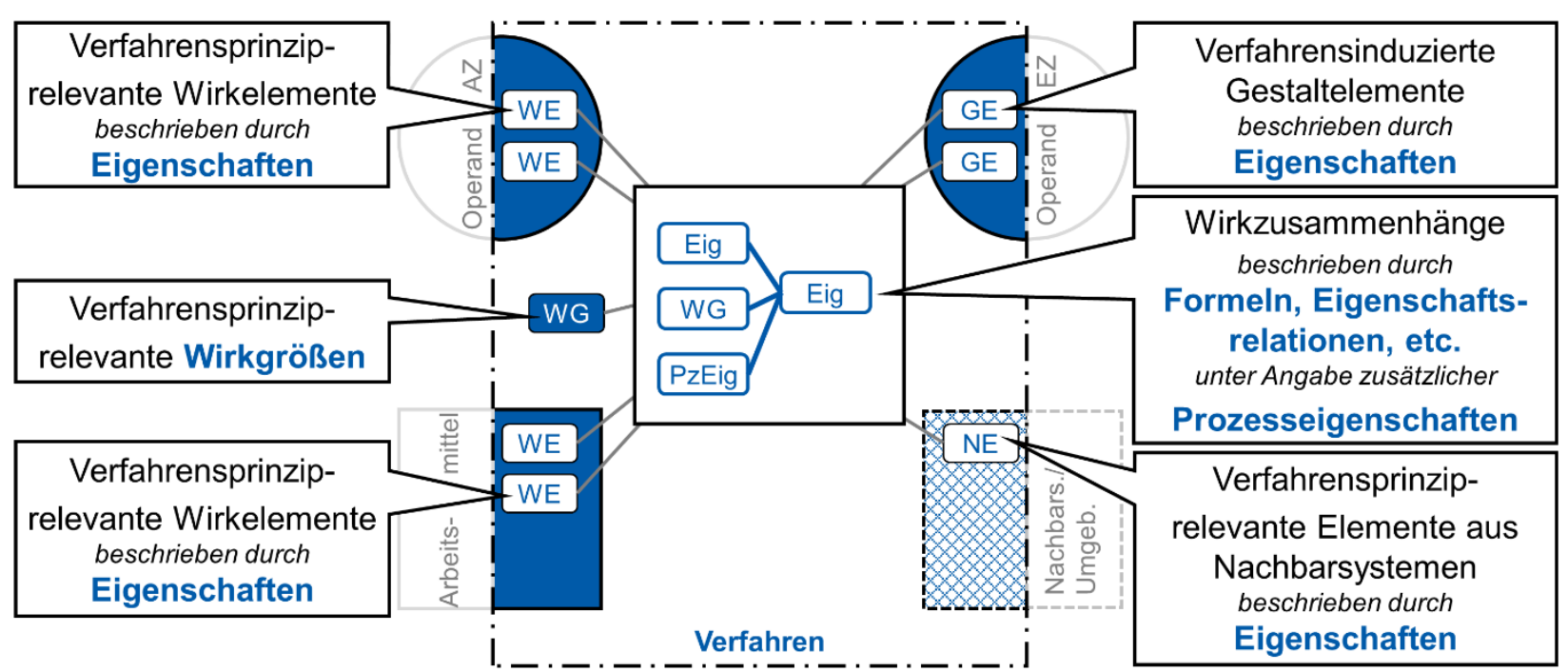

Abbildung 4-10: Verfahrensprinzipmodell (VP-Modell) 
GRAMLICH zeigt bereits eine Möglichkeit auf, die Modellelemente des Verfahrensprinzips einheitlich mittels Eigenschaften zu beschreiben. Dies umfasst verfahrensprinziprelevante Wirkelemente, die sowohl dem Operanden als auch dem Arbeitsmittel zugeordnet sein können, verfahrensprinziprelevante Wirkgrößen, die die Zustandsänderung im eigentlichen Sinne bewirken, und verfahrensinduzierte Gestaltelemente, die das Ergebnis der Zustandsänderung beschreiben. Sämtliche dieser Elemente werden durch entsprechend benannte Eigenschaften beschrieben. Die aus Abschnitt 4.2.1 bekannten verfahrensrelevanten Eigenschaften gehen in den verfahrensprinziprelevanten Eigenschaften der Wirkelemente des Operanden im Anfangszustand auf. Für die Beschreibung der Wirkgrößen gilt, dass diese formal wie Eigenschaften gehandhabt werden können. Hinzu kommen Elemente, die weder dem Operanden noch dem Arbeitsmittel zugeordnet werden können. Sie sind Teil von Nachbarsystemen, die zwar außerhalb der Systemgrenze des technischen Prozesses liegen, aber dennoch derart auf den Prozess Einfluss nehmen, dass sie in der Modellierung mitbetrachtet werden müssen. Sie werden dementsprechend verfahrensprinziprelevante Nachbarelemente genannt und durch verfahrensprinziprelevante Eigenschaften beschrieben. Sie kommen erfahrungsgemäß nur selten vor, sodass sie in den folgenden Ausführungen eine untergeordnete Rolle spielen. Exemplarisch seien hier Verfahren wie das Kleben genannt. Dort spielen insbesondere die Umgebungstemperatur und die Luftfeuchtigkeit eine wesentliche Rolle für die Qualität der Klebung und die Aushärtedauer. ${ }^{190}$ Können diese Einflüsse nicht direkt dem Operanden oder dem Arbeitsmittel zugeordnet werden, so bietet sich die Möglichkeit, diese den Nachbarsystemen der Prozessumgebung zuzuordnen.

Da sich das Arbeitsmittel per Definition mit zunehmender Prozessdauer nicht ändert ${ }^{191}$, stehen nur die Wirkgrößen zur Abbildung zeitlicher Einflüsse zur Verfügung. Diese stellen keine stofflichen Gebilde dar und können somit nicht durch Zustände beschrieben werden. Trotzdem können sich die Wirkgrößen mit der Zeit ändern bzw. in Abhängigkeit der Prozesszeit beschrieben werden. Abbildung 4-11 zeigt dies am Beispiel des Spaltprofilierens. Aufgrund der Spaltwalzengeometrie und des kontinuierlichen Blechvorschubs wird die Kontaktkraft zwischen Spaltwalze und Blechband nicht sprunghaft aufgebracht. Innerhalb des Kontaktbereichs

190 Vgl. Habenicht (2002), S. 512 f.

191 Vgl. Gramlich (2013), S. 93. 
zwischen Spaltwalze und Blechband (Eingriff bis Auslauf) ändert sich die Kraftangriffsrichtung und $u$. a. mit dem zu verdrängenden Materialvolumen auch der Betrag der Kraft. Die Wirkgröße ist somit durch einen zeitlich und örtlich veränderlichen Verlauf charakterisiert. ${ }^{192}$

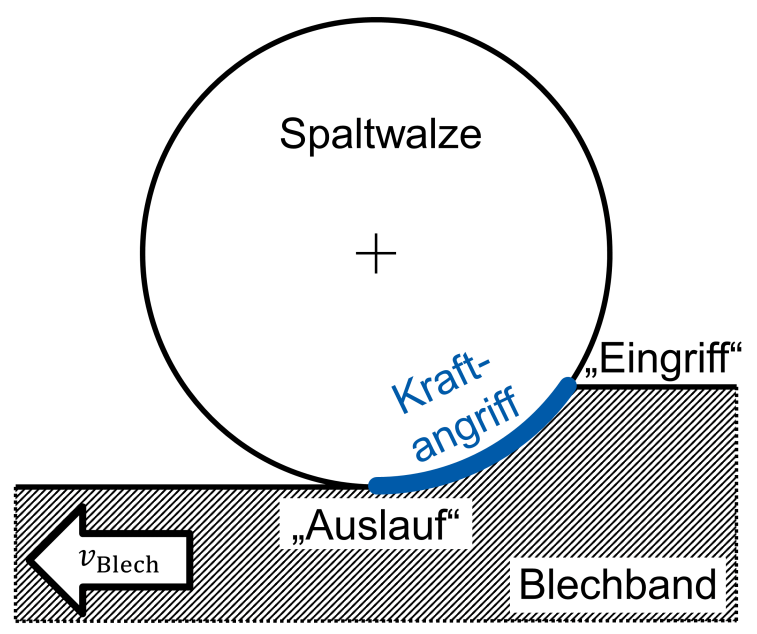

Abbildung 4-11: Kontaktbereich beim Spaltprofilieren ${ }^{193}$

Die wesentliche Neuerung des VP-Modells (siehe Abbildung 4-10) liegt in der Abbildung von Wirkzusammenhängen. Diese werden durch in Relation stehende Eigenschaften der am Prozess beteiligten stofflichen Systeme und Größen beschrieben. Zur Abbildung der Eigenschaftsrelationen können mathematische Formeln, Abbildungen, Diagramme, Netzwerke, etc. verwendet werden, solange diese einen Rückschluss auf die im Zusammenhang stehenden Eigenschaften zulassen. Dabei können zusätzliche Prozesseigenschaften ${ }^{194}$ angegeben werden, die nicht als Eigenschaften der am Prozess beteiligten Objekte erfasst werden können aber dennoch für eine vollständige Beschreibung der Wirkzusammenhänge notwendig sind. Ein Beispiel für Prozesseigenschaften sind Reibungskoeffizienten. Diese können weder den Wirkelementen des Operanden noch denen des Arbeitsmittels eindeutig zugeordnet werden. Sie ergeben sich erst durch das Zusammenwirken mehrerer Wirkflächen. Ebenso wie Produkteigenschaften können diese als Kombination aus Merkmal und Ausprägung dokumentiert werden. Für die auftretende Reibung kann das Merkmal Reibungskoeffizient $\mu$ beispielsweise durch die Ausprägung 0,1 spezifiziert werden. Prozesseigenschaften können darüber hinaus auch das Delta der Zustandsänderung zwischen Anfangszustand und Endzustand

\footnotetext{
192 Vgl. Jöckel (2005), S. 55.

193 In Anlehnung an Jöckel (2005), S. 41.

194 In Anlehnung an Röder et al. (2012), S. 11.
} 
des Operanden beschreiben. Das Aufweiten von Rohren im Rahmen des Innenhochdruckumformens kann so z. B. als Durchmesseränderung $\Delta D$ erfasst werden. Als Prozesseigenschaft beschreibt sie weder den Operanden im Anfangs- noch im Endzustand. Sie ergibt sich als Differenz der Rohrdurchmesser in den Zuständen.

Das VP-Modell erlaubt es, fertigungstechnologisches Produkt-Wissen in der Form von Eigenschaftsrelationen umfassend abzubilden. Auch sehr unterschiedlich dokumentiertes Wissen kann durch Verwendung des Modells auf seine Aussagen in Bezug auf Wirkelemente und deren Eigenschaften untersucht werden. Das VPModell mit seinen Elementen und Eigenschaften kann als Checkliste verwendet werden, um aus der Summe von Prozessparametern Eigenschaften zu identifizieren, die eindeutig die beteiligten technischen Objekte beschreiben, sowie die Wirkgrößen, die für die Durchführung des Prozesses unabdingbar sind. Diese stellen gleichzeitig zentrale Informationen für die Produkt- und Prozessentwicklung dar.

\section{Modellierung des Verfahrensprinzips für das Innenhochdruckumformen}

Abbildung 4-12 zeigt das VP-Modell für das Innenhochdruckumformen. Die grafische Darstellung im Zentrum beinhaltet alle relevanten Wirkelemente, Gestaltelemente und Wirkgrößen und gibt an, wie diese zusammenwirken.

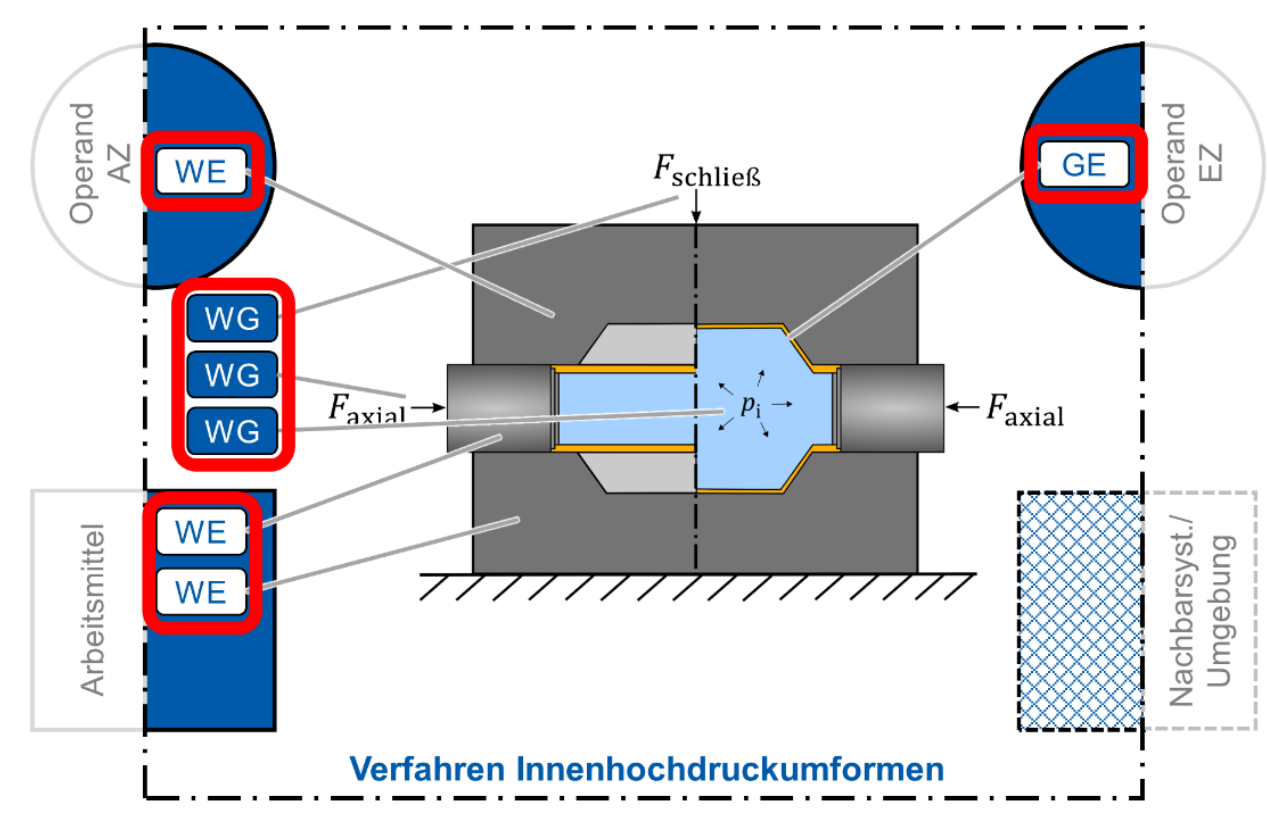

Abbildung 4-12: VP-Modell für das Innenhochdruckumformen ${ }^{195}$

195 In Auszügen aus Noack, Sterzing (2007), S. 15. 
Die Einordnung der grafischen Darstellung des Innenhochdruckumformens in das Modell ermöglicht die konkrete Zuordnung zu den am Verfahren beteiligten Objekten, welche auf diesem Weg wiederum in einen konkreten Wirkzusammenhang gestellt werden. Bei der dargestellten Grafik des Verfahrensprinzips handelt es sich um die abstrakteste Darstellung des Wirkzusammenhangs. Sie gibt noch keine Auskunft darüber, in welcher Form die einzelnen Elemente genau miteinander interagieren bzw. wie darauf Einfluss genommen werden kann.

Einen konkreten Einblick in das Verfahrensprinzip des Innenhochdruckumformens gewährt Abbildung 4-13. Darin werden nur diejenigen Wirkzusammenhänge dargestellt, deren Fokus auf der Ausbildung bestimmter Domhöhen beim Innenhochdruckumformen zylindrischer Rohre liegt. Demnach besteht ein klarer Zusammenhang zwischen dem Durchmesser des Rohres (Wirkelement des Operanden), dem Stempelabstand des Werkzeugs (Wirkelement des Arbeitsmittels) und der realisierbaren Domhöhe (verfahrensinduziertes Gestaltelement).

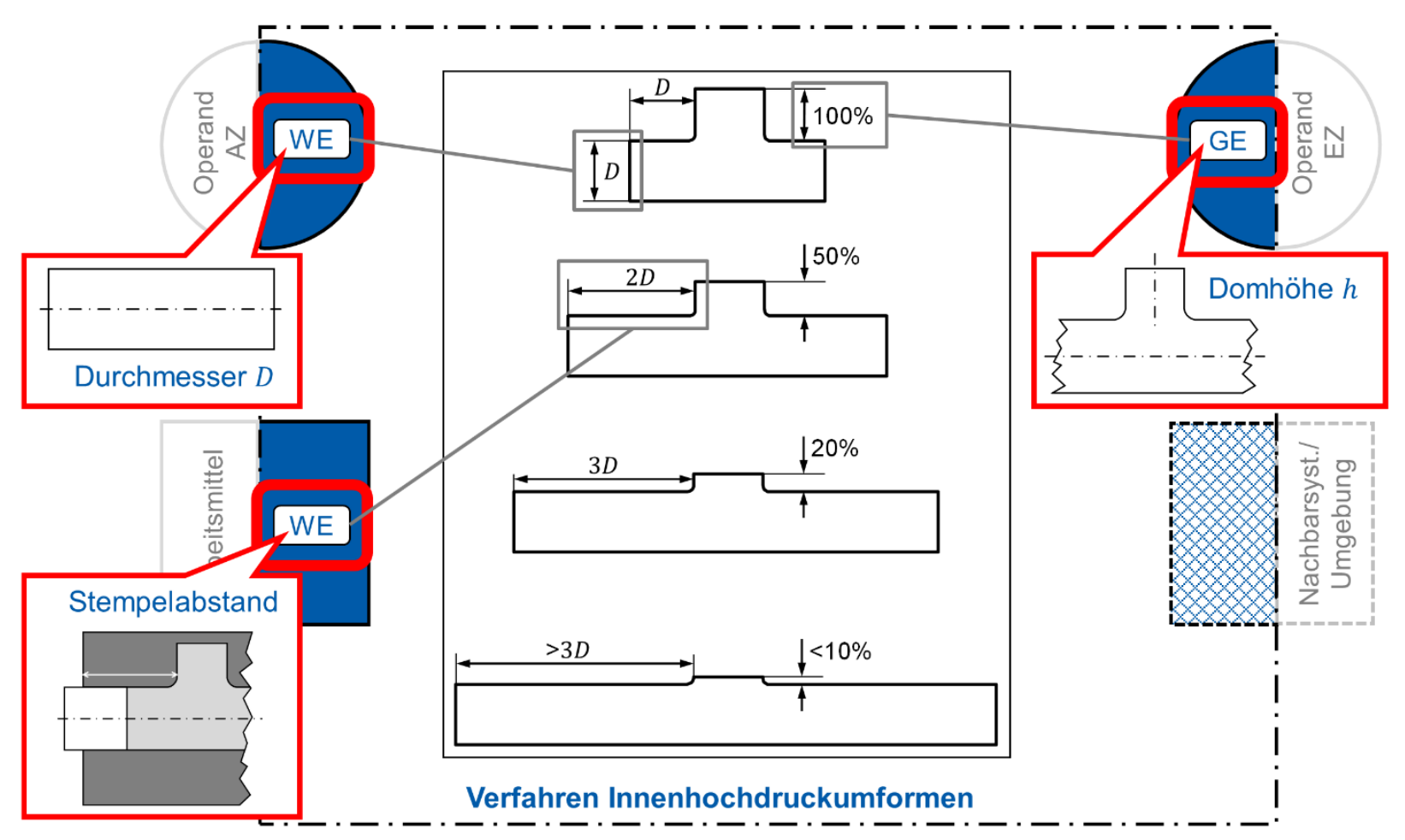

Abbildung 4-13: Domhöheneinflüsse im VP-Modell für das Innenhochdruckumformen ${ }^{196}$

Abbildung 4-13 verdeutlicht, dass nicht immer eine mathematische Formel notwendig ist, um Wirkzusammenhänge eindeutig zu beschreiben. Aus dem Ver-

196 In Auszügen aus Bahn et al. (2007), S. 43. 
gleich zur Abbildung 4-12 wird offensichtlich, dass das VP-Modell das Verfahrensprinzip nicht zwangsweise vollumfänglich abbilden muss. Durch seinen Checklistencharakter kann das VP-Modell zur Abbildung sehr unterschiedlicher Dokumentationsformen und Granularitätsstufen fertigungstechnologischen Produkt-Wissens eingesetzt werden, sodass stets eine zweckmäßige Modellierung erleichtert wird.

Die Notwendigkeit, fertigungstechnologisches Produkt-Wissen in Form eines einheitlichen Modells nutzbar zu machen, zeigt sich gerade am Beispiel der Domhöhe. Intuitiv würde ein Entwickler von einer größer werdenden Domhöhe ausgehen, wenn das Rohrhalbzeug länger ist und somit mehr Material zur Verfügung steht, das beim Umformen nachfließen kann. Tatsächlich werden die realisierbaren Domhöhen im realen Fertigungsprozess mit steigender Rohrlänge kleiner. Auch wenn der Entwickler die erklärenden Mechanismen nicht im Detail verstehen muss, so kann er durch den Zugriff auf das im VP-Modell abgebildete Wissen entsprechende Rückschlüsse für die Konstruktion seiner innenhochdruckumgeformten Bauteile ziehen.

Abbildung 4-14 zeigt, wie sich der zulässige Innendruck $p_{\text {i zul }}$ und das verwendete Halbzeug mit seinen Eigenschaften Durchmesser $D_{0}$, Wanddicke $s_{0}$ und Festigkeit $R_{\mathrm{m}}$ gegenseitig beeinflussen. Die Wahl des Halbzeugs hat damit einen wesentlichen Einfluss auf die maximal ertragbare Ausprägung der Wirkgröße.

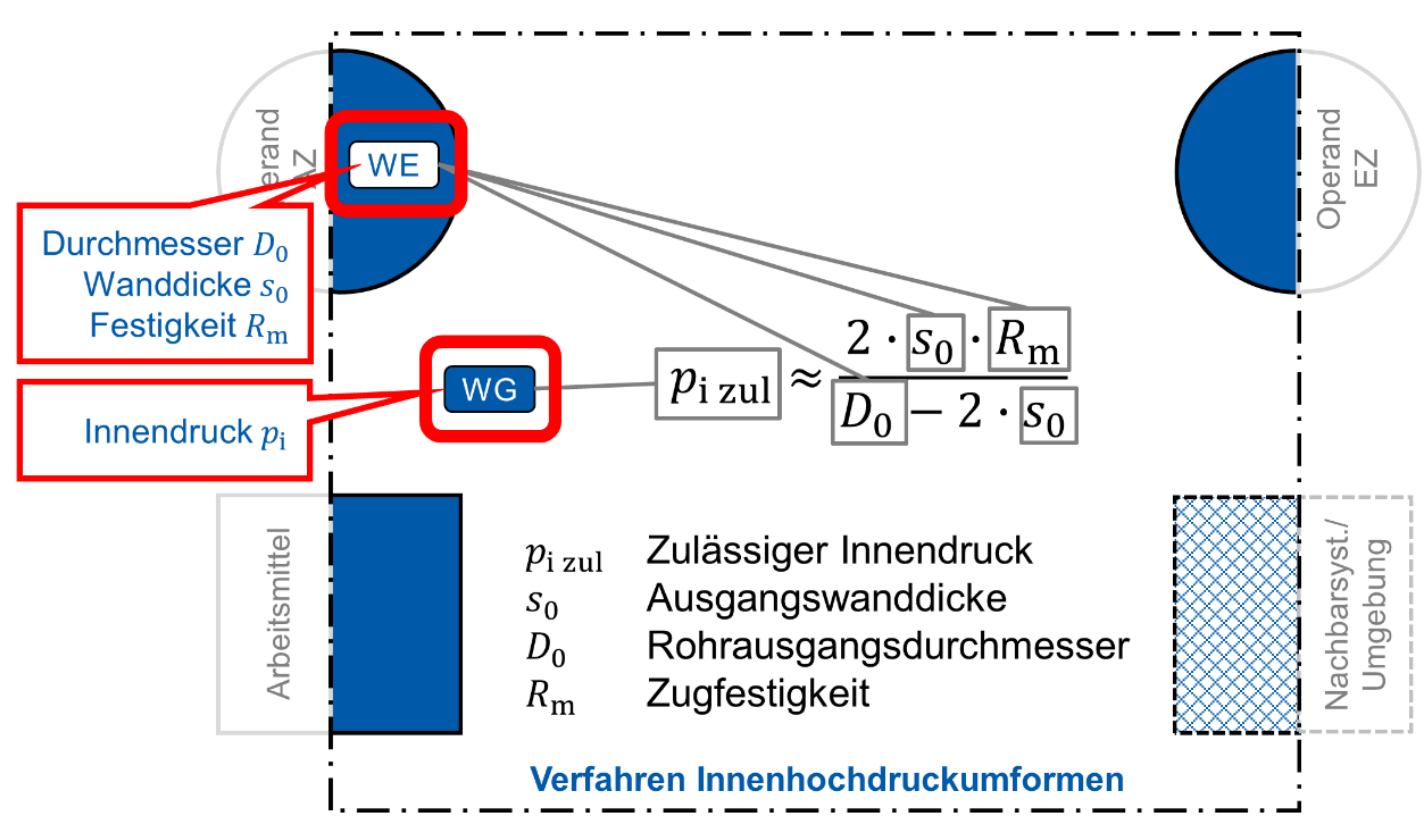

Abbildung 4-14: Zulässiger Innendruck im VP-Modell für das Innenhochdruckumformen 
Gerade Wirkzusammenhänge, die in der Form mathematischer Formeln dokumentiert sind, können sehr leicht mittels Eigenschaftsnetzwerken dargestellt werden, um die Abhängigkeiten der Eigenschaften untereinander deutlicher hervorzuheben (siehe Abbildung 4-15). Das VP-Modell entspricht somit in Bezug auf Detailgrad und Formalismus den Modellen der Produktmodellierung, die ebenso mittels Eigenschaften beschrieben werden können.

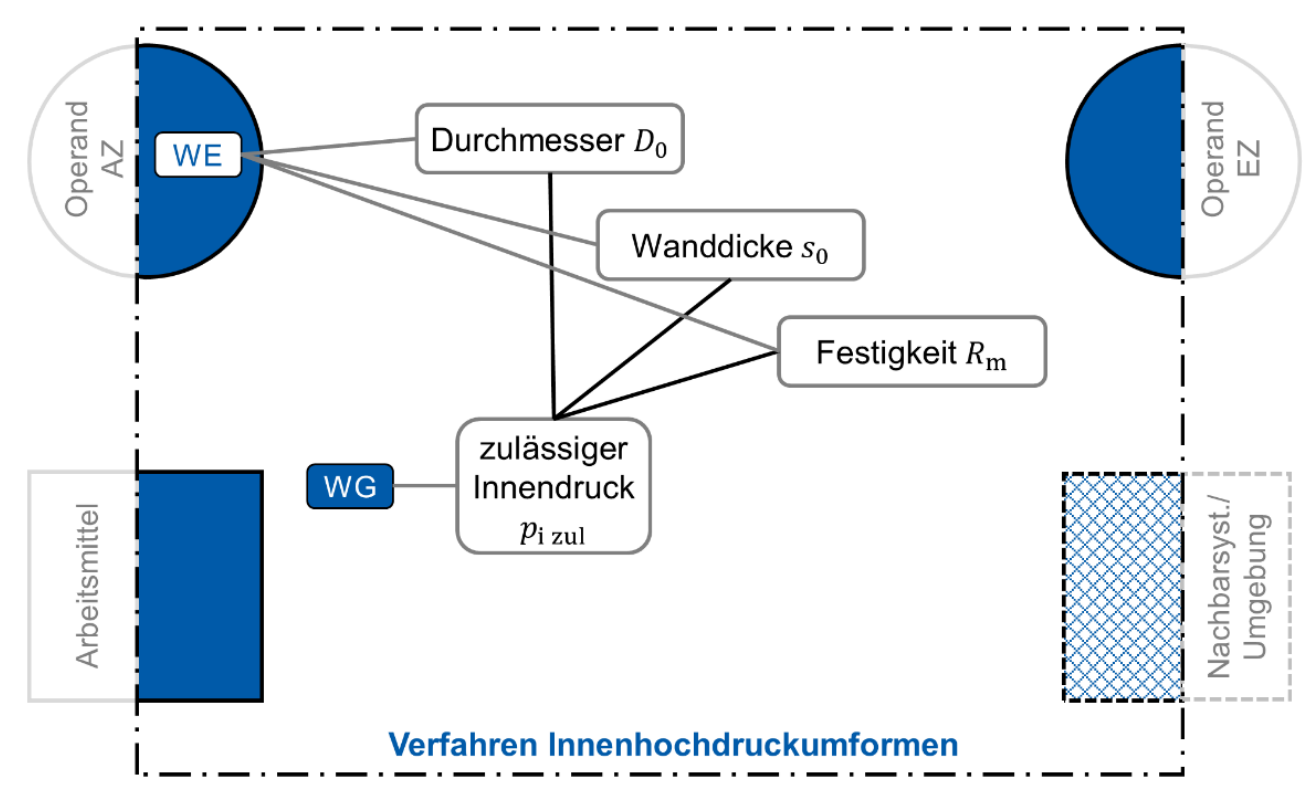

Abbildung 4-15: Zulässiger Innendruck im VP-Modell für das Innenhochdruckumformen in Form eines Eigenschaftsnetzwerks

\section{Modellierung des Verfahrensprinzips für das Spaltprofilieren}

Auch für das Spaltprofilieren existiert eine allgemeine Darstellung des Verfahrensprinzips. Wird diese in das VP-Modell implementiert, so können die wesentlichen Wirkelemente, Gestaltelemente und Wirkgrößen identifiziert werden, ohne die das Verfahren nicht beschrieben werden kann (siehe Abbildung 4-16). Vor allem die Wirkelemente des Arbeitsmittels sind charakteristisch für das Verfahrensprinzip. Spalt- und Hilfswalzen sind so angeordnet, dass ihre Außenflächen eine Art Kanal bilden, durch den das Material des Operanden während der Umformung fließen kann. Der Operand muss dazu ein Blechhalbzeug mit gerader Blechkante sein. Die eigentliche Umformung wird durch die Wirkgrößen Spaltwalzenkraft $F$ und Blechvorschub $v_{\text {Blech }}$ bewirkt. Durch die Umformung werden charakteristische Gestaltelemente ausgebildet: Zwei Flansche an jeder Bandkante und ein verkürzter Stegbereich. Diese können über zusätzliche Eigenschaften, die teils in Form von Graphen vorliegen, näher beschrieben werden. 


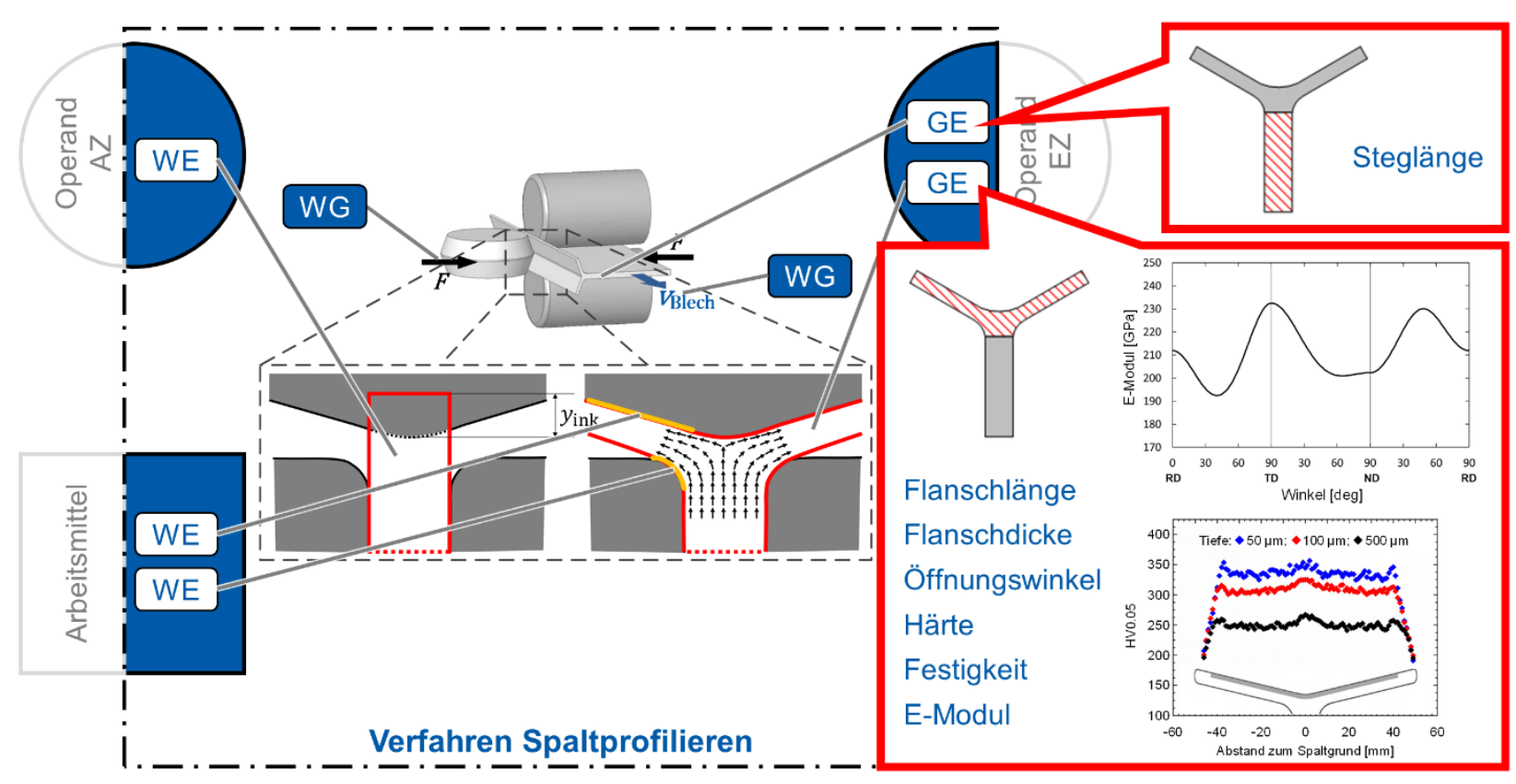

Abbildung 4-16: VP-Modell für das Spaltprofilieren ${ }^{197}$

Auch Wirkzusammenhänge, die mittels Diagrammen dokumentiert sind, können durch das VP-Modell abgebildet werden. Abbildung 4-17 zeigt Verläufe für die Reaktionskräfte an der Spaltwalze. Die Höhe der Spaltwalzenkräfte (Wirkgröße) lässt sich durch den Flankenwinkel an der Spaltwalze (Wirkelement) beeinflussen.

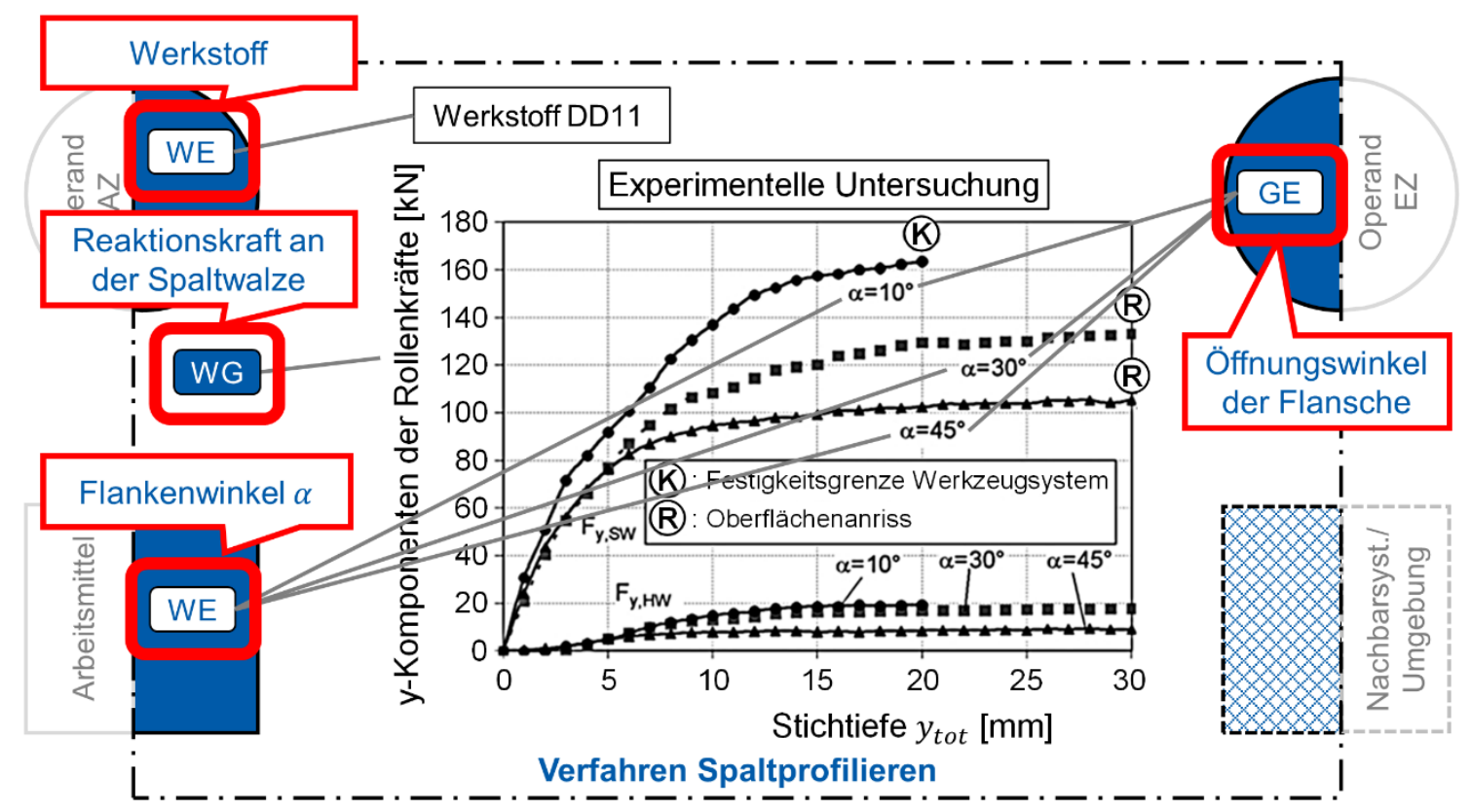

Abbildung 4-17: Spaltwalzenkräfte im VP-Modell für das Spaltprofilieren ${ }^{198}$

197 In Auszügen aus Gramlich (2013), S. 88; Ahmels et al. (2017), S. 108, 111.

198 In Auszügen aus Groche et al. (2007), S. 254. 
Durch die Angabe des Flankenwinkels ist implizit auch der theoretisch erreichbare Öffnungswinkel des Spaltprofils im Endzustand definiert. Ein Flankenwinkel von $10^{\circ}$ hat im Idealfall einen Öffnungswinkel von $160^{\circ}$ zur Folge. Der geometrische Parameter $y_{\text {tot }}$ ist keinem der am Verfahren beteiligten Objekte eindeutig zuzuweisen. Er ist vielmehr eine Prozesseigenschaft, die die virtuelle Überschneidung des Operanden im Anfangszustand mit dem Arbeitsmittel beschreibt. Erst die aus dieser geometrischen Überschneidung resultierende Reaktionskraft an der Spaltwalze ist die eigentliche Wirkgröße, die den hydrostatischen Spannungszustand bei der Umformung erzeugt und damit das Verfahrensprinzip charakterisiert.

Das Verfahrensprinzip des Spaltprofilierens ist auch dadurch gekennzeichnet, dass die erreichbare Oberflächenrauheit der Flansche wesentlich durch die Oberflächenrauheit des Werkzeugsystems bestimmt wird. Die entsprechenden Zusammenhänge sind in Form von Balkendiagrammen dokumentiert (siehe Abbildung 4-18). Auch dieses fertigungstechnologische Produkt-Wissen kann unter Zuhilfenahme des VP-Modells abgebildet und die darin verknüpften Wirk- und Gestaltelemente eindeutig identifiziert werden.

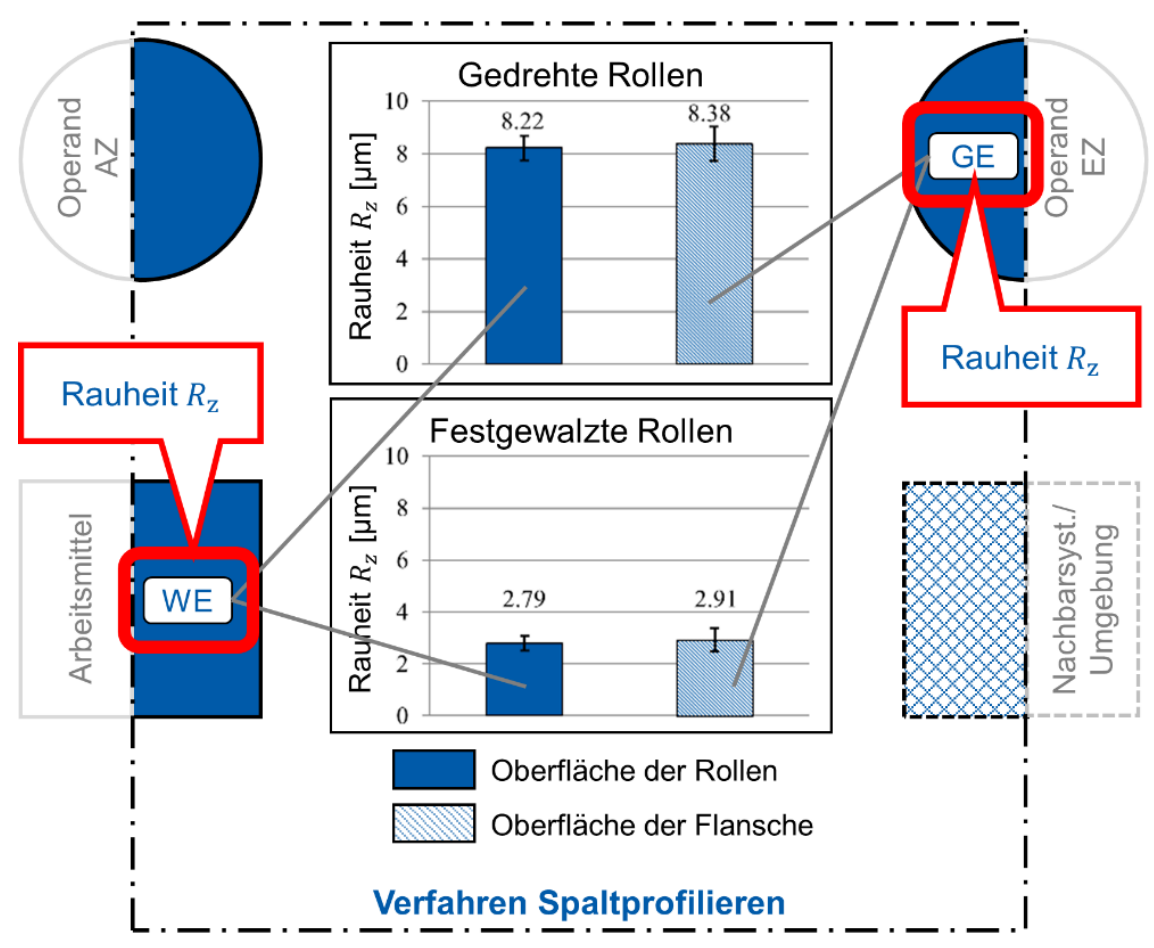

Abbildung 4-18: Oberflächenrauheit im VP-Modell für das Spaltprofilieren ${ }^{199}$

199 In Auszügen aus Monnerjahn, Fricke (2012). 
Die angeführten Beispiele zeigen deutlich, dass trotz der großen Heterogenität dokumentierten fertigungstechnologischen Wissens, stets eindeutig herausgearbeitet werden kann, welche Informationen den einzelnen am Prozess beteiligten Objekten zugeordnet werden muss. Weitere Beispiele finden sich im Anhang B. Das Modell ist über seinen reinen Abbildungszweck von Wirkzusammenhängen hinaus auch eine Checkliste, um Wirkelemente, Gestaltelemente, deren Eigenschaften und Wirkgrößen zu identifizieren. Die Schlagkraft des Modells liegt darin, dass mit seiner Hilfe beliebig dokumentiertes fertigungstechnologisches Wissen (z. B. mittels mathematischer Formeln, Abbildungen, textueller Auszüge, Graphen, etc.) auf zentrale Aussagen hinsichtlich der beteiligten technischen Produkte untersucht werden kann. Somit kann geklärt werden, inwiefern dieses Wissen die zu entwickelnden Produkte - sei es das Arbeitsmittel oder der Operand des jeweiligen Prozesses - betrifft. Das Prozessmodell auf Basis des Verfahrensprinzips stellt damit ein Werkzeug zur Abbildung und Analyse fertigungstechnologischen Wissens mit Blick auf die Produktentwicklung dar.

\subsection{Vorgehen zum Konkretisieren technischer Produkte im Kontext des Einsatzprozesses und der Produktfunktion}

Im Fokus der Produktentwicklung steht vor allem die Synthese technischer Produkte mit dem Ziel, einen möglichst hohen Kundennutzen durch das Produkt bereitzustellen. Ein entsprechendes Vorgehen erfordert nicht nur die Konkretisierung der Produktfunktion, sondern auch die Konkretisierung des Produkts im Kontext seiner Einsatzprozesse. Hierzu werden Modelle benötigt, mittels derer Einsatzprozesse ebenso konsistent modelliert werden können, wie das technische Produkt.

In Abschnitt 2.4.3 wurde bereits gezeigt, dass Fertigungs- und Einsatzprozesse anhand ihrer Rolle im Produktlebenslauf grundlegend voneinander zu unterscheiden sind. In Einsatzprozessen fungiert das Produkt als Arbeitsmittel, wohingegen es in Fertigungsprozessen als Operand eine Zustandsänderung erfährt. Dennoch existiert eine wesentliche Gemeinsamkeit zwischen Fertigungs- und Einsatzprozessen. Beide beschreiben eine Zustandsänderung die durch ein Arbeitsmittel realisiert wird. Abbildung 4-19 zeigt hierzu das Bespiel eines Bolzens, der durch Einsatz eines geeigneten Werkzeugsystems gehärtet wird. Auf den ersten Blick ist darin ein Fertigungsprozess dargestellt, der die gewollte Zustandsänderung unter Berücksichtigung des Arbeitsmittels darstellt. Auf den zweiten Blick kann darin auch ein Einsatzprozess gesehen werden. Das Werkzeugsystem fungiert als Ar- 
beitsmittel und erfährt daher selbst keine gewollte Zustandsänderung. Damit befindet sich das Werkzeugsystem, als technisches Produkt, in einem Einsatzprozess, in dem es selbst nicht der Operand des Prozesses ist.

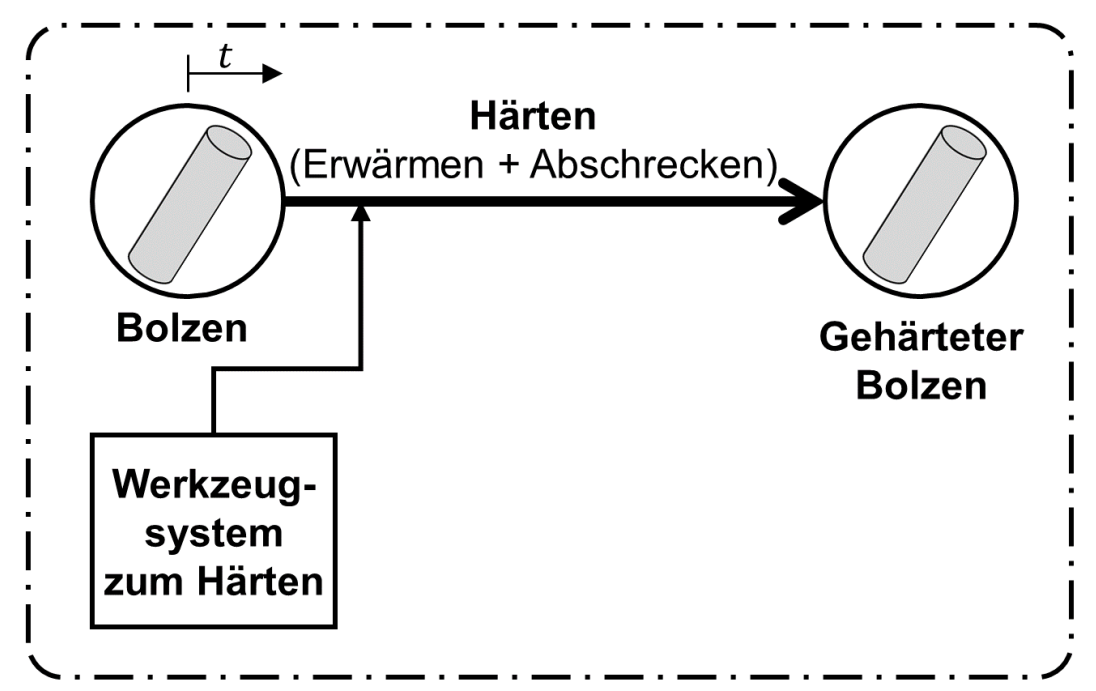

Abbildung 4-19: Härten von Bolzen unter Berücksichtigung des Arbeitsmittels

Es hängt somit vom Blickwinkel auf den technischen Prozess ab, ob er als Einsatzprozess des Arbeitsmittels oder als Fertigungsprozess des Operanden betrachtet wird. Damit können die Modelle der vorangegangenen Abschnitte auch für die Konkretisierung von Einsatzprozessen verwendet werden, in denen das Arbeitsmittel im Fokus der Betrachtungen steht. Abbildung 4-20 zeigt die verschiedenen Konkretisierungsebenen, auf denen diese Modelle verwendet werden können, im Vergleich zu den Konkretisierungsebenen der Produktmodellierung.
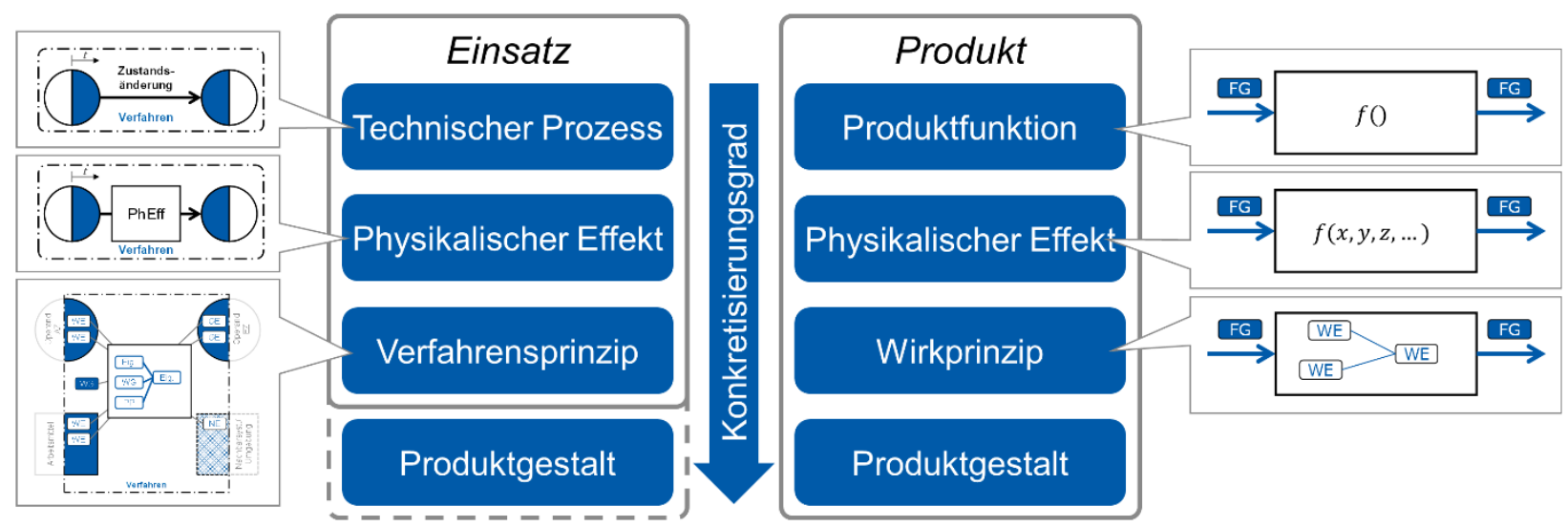

Abbildung 4-20: Modellebenen der Einsatzprozess- und Produktkonkretisierung im Rahmen der Produktentwicklung ${ }^{200}$

200 In Anlehnung an Gramlich (2013), S. 93. 
Die abstrakteste Modellierungsebene der Produktmodellierung bildet die Produktfunktion. Auf den konkreteren Ebenen werden in Anknüpfung an die Produktmodellpyramide nach SAUER physikalische Effekte und Wirkprinzipien modelliert, die eine konkretere Beschreibung der Produktfunktion ermöglichen, indem sie auf Zusammenhänge zurückgreifen, die die Eingangs- und Ausgangsfunktionsgrößen miteinander in Bezug setzen. ${ }^{201}$ Die Konkretisierung des Produkts mündet in der Festlegung der Produktgestalt auf der untersten Produktmodellebene. Die festgelegte Produktgestalt stellt gleichermaßen die konkreteste Modellebene auf der Seite der (Einsatz-)Prozessmodellierung dar. Das Ziel der Konkretisierung von Einsatzprozessen im Rahmen der Produktentwicklung liegt ebenfalls darin, einen möglichst hohen Kundennutzen durch das Produkt bereitzustellen. Dennoch erfordert die einsatzprozessseitige Konkretisierung des Produkts auch die Konkretisierung der Einsatzprozesse selbst, in denen das Produkt als Arbeitsmittel fungiert.

Die Ausführungen der vorherigen Abschnitte zeigen, dass es nicht ein einheitliches allumfassendes Prozessmodell gibt, mit dem technische Prozesse abgebildet werden können. Je nach Modellierungszweck und Konkretisierungsgrad können unterschiedliche Modelle verwendet werden. So bietet die Modellierung der Zustandsänderung (Modellebene „Technischer Prozess“) die Möglichkeit, zeitliche Abfolgen und auch Prozessstrukturen näher zu betrachten. Als abstrakteste Modellebene des Einsatzprozesses wird darin der eigentliche Zweck beschrieben, den der Kunde mit dem angedachten Produkt erreichen möchte. Nach der Konkretisierung mittels physikalischer Effekte wird das zu entwickelnde Produkt als Arbeitsmittel des Einsatzprozesses erst auf der Ebene des Verfahrensprinzips konkret in die Entwicklung einbezogen. Durch Verwendung des VP-Modells können Wirkzusammenhänge abgebildet und Schlussfolgerungen für die Gestalt des Arbeitsmittels bzw. die Produktgestalt gezogen werden. Da alle verwendeten Modelle auf der Beschreibung mittels Eigenschaften beruhen, ist eine modellübergreifende Konkretisierung des technischen Produkts und des Einsatzprozesses möglich. Auf dieser Basis können die in Abbildung 4-20 dargestellten Modelle in Abhängigkeit ihres Konkretisierungsgrades in ein entsprechendes Vorgehen zur Produkt- und Prozesskonkretisierung eingebunden werden (siehe Abbildung 4-21).

201 Eine detaillierte Beschreibung findet sich in Gramlich (2013), S. $120 \mathrm{ff}$. 


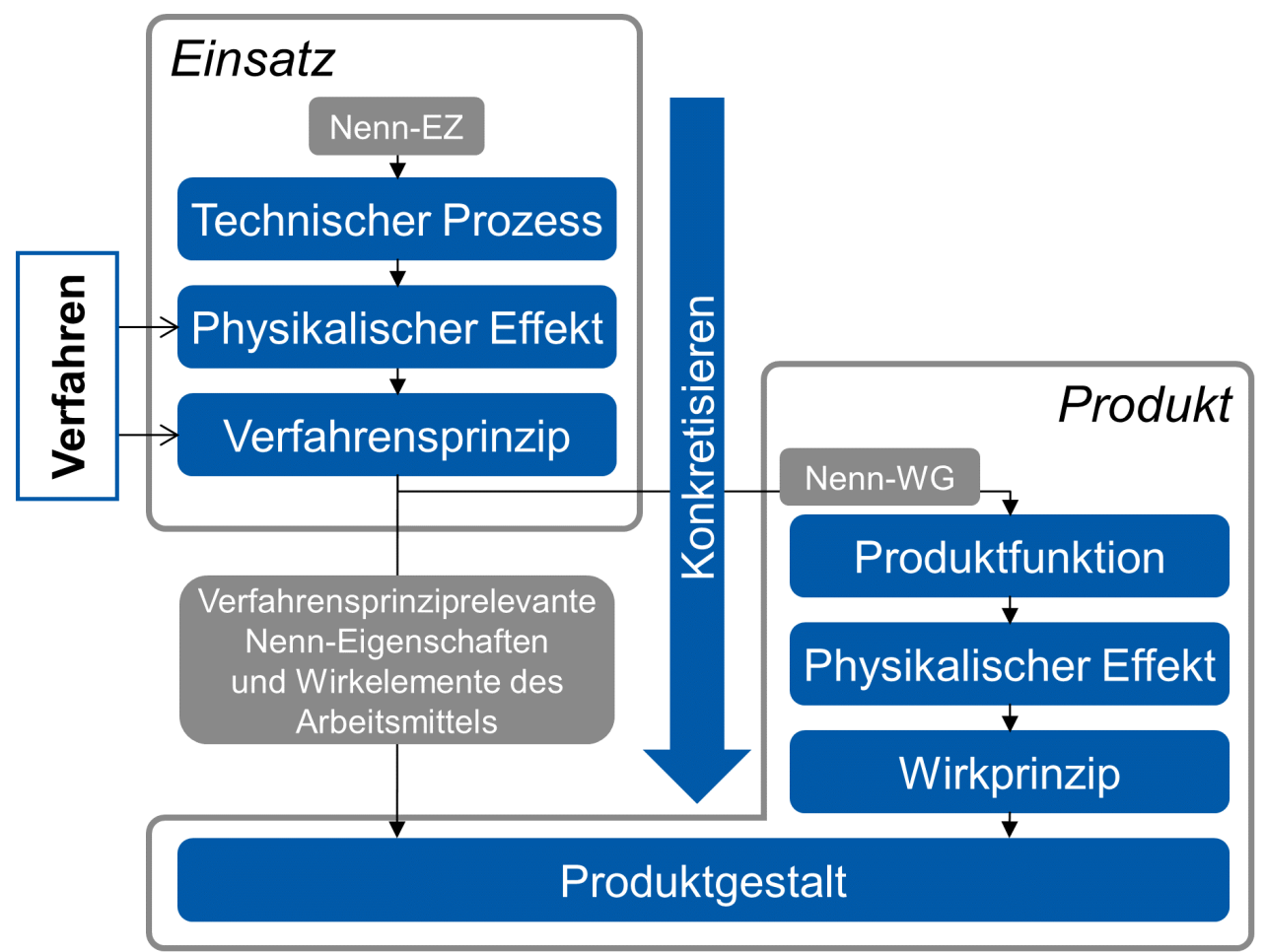

\section{Abbildung 4-21: Vorgehen zur Produkt- und Einsatzprozesskonkretisierung im Rahmen der Produktentwicklung}

Um das Vorgehen aus Abbildung 4-21 konkret darzulegen wird im Folgenden das bereits vorgestellte Beispiel eines Härteprozesses aufgegriffen. Ziel ist es, das Arbeitsmittel dieses Prozesses bis hin zu einer festgelegten Produktgestalt zu konkretisieren. Der Zweck des Einsatzprozesses ist es, einen gehärteten Bolzen zu realisieren, der den Nenn-Endzustand (Nenn-EZ) der Prozesskette aus Erwärmen und Abschrecken charakterisiert (siehe Abbildung 4-22). Der Nenn-Endzustand des Operanden wird durch Nenn-Eigenschaften ${ }^{202}$ beschrieben. Im ersten Schritt werden geeignete Verfahren festgelegt, mittels derer die Zustandsänderung realisiert wird. Abbildung 4-22 zeigt exemplarisch zwei Verfahrensalternativen Flammhärten und Induktionshärten. Die exemplarisch dargelegten Verfahrensalternativen unterscheiden sich vor allem im Teilprozess „Erwärmen“, in denen das Verfahren auf einem vollkommen anderen physikalischen Effekt beruht. Das Flammhärten erwärmt das Werkstück mittels eines Wärmestroms, der aus der Verbrennung eines Gasgemisches resultiert. Das Induktionshärten basiert hingegen auf dem Erzeugen von Wärme infolge von Wirbelströmen im Werkstück, die durch ein hochfrequentes elektrisches Wechselfeld hervorgerufen werden. ${ }^{203}$

\footnotetext{
202 Eine ausführliche Differenzierung von Soll-, Nenn- und Ist-Eigenschaften findet sich in Gramlich (2013), S. $117 \mathrm{ff}$.

${ }^{203}$ Vgl. Seidel, Hahn (2010), S. $143 \mathrm{ff.}$
} 

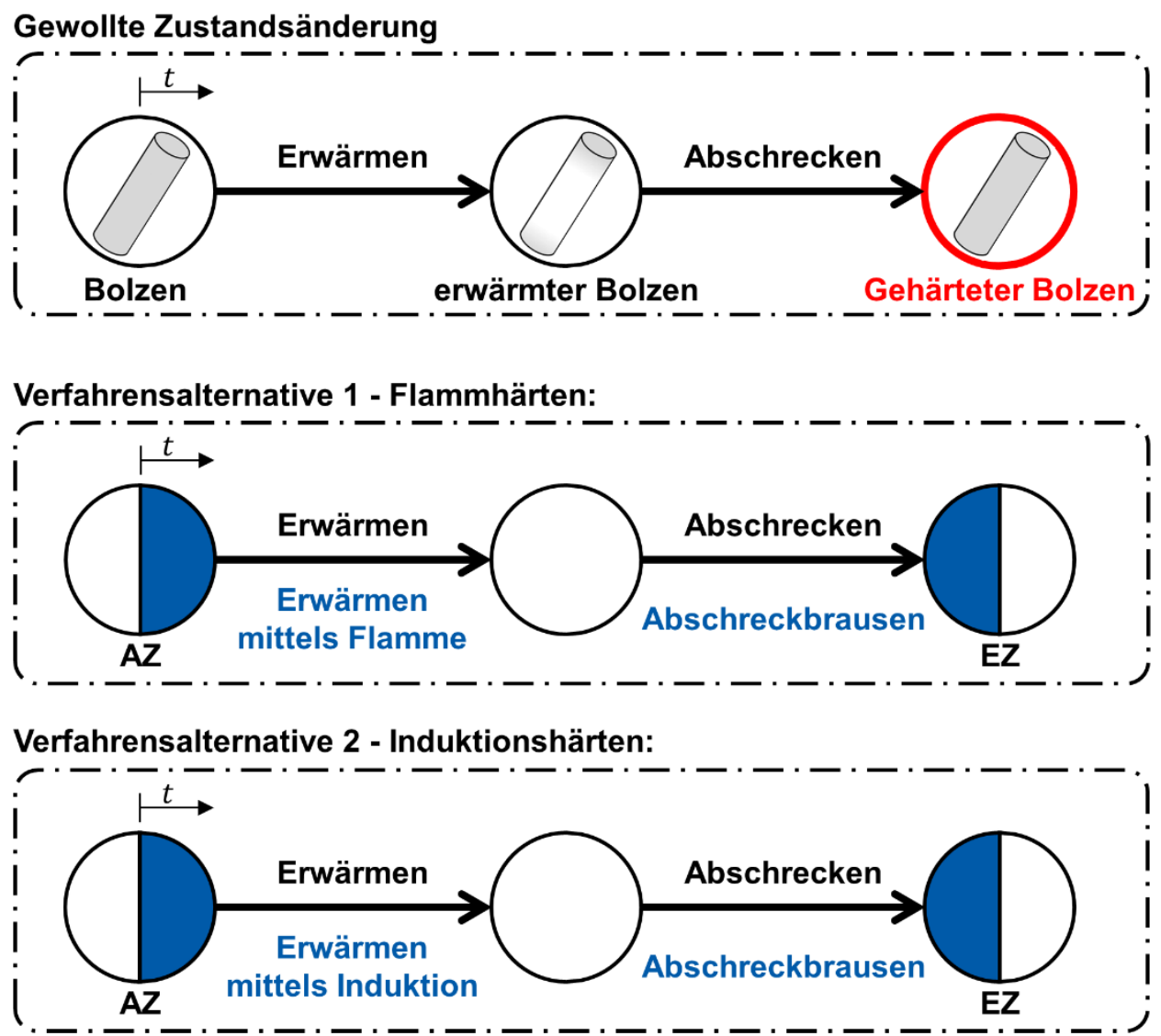

\section{Abbildung 4-22: Gewollte Zustandsänderung mit Nenn-Endzustand des Einsatzprozesses mit Verfahrensalternativen Flammhärten und Induktionshärten}

Beide Verfahrensalternativen können im nächsten Schritt durch Angabe des Verfahrensprinzips konkretisiert werden. Abbildung 4-23 und Abbildung 4-24 zeigen dazu das VP-Modell mittels dessen beide Verfahrensprinzipien dargestellt werden können. Es zeigt sich, dass sich das Flammhärten und das Induktionshärten vor allem im Arbeitsmittel und der durch dieses bereitgestellten Wirkgröße unterscheiden. Während das Flammhärten einen Wärmestrom $\dot{Q}$ mittels eines hitzebeständigen Werkzeugs dem Werkstück zuführt, basiert das Induktionshärten auf dem Vorhandensein eines elektrischen Wechselfeldes mit der magnetischen Energie $E_{\text {magn }}$. Das Arbeitsmittel bzw. dessen Wirkelement muss ringförmig um den Bolzen angeordnet sein, um eine möglichst gleichmäßige Erwärmung zu erreichen. Das jeweilige Verfahren gibt somit vor, wie der Einsatzprozess konkretisiert wird und welche Wirkgröße durch das Arbeitsmittel bereitgestellt werden muss. 


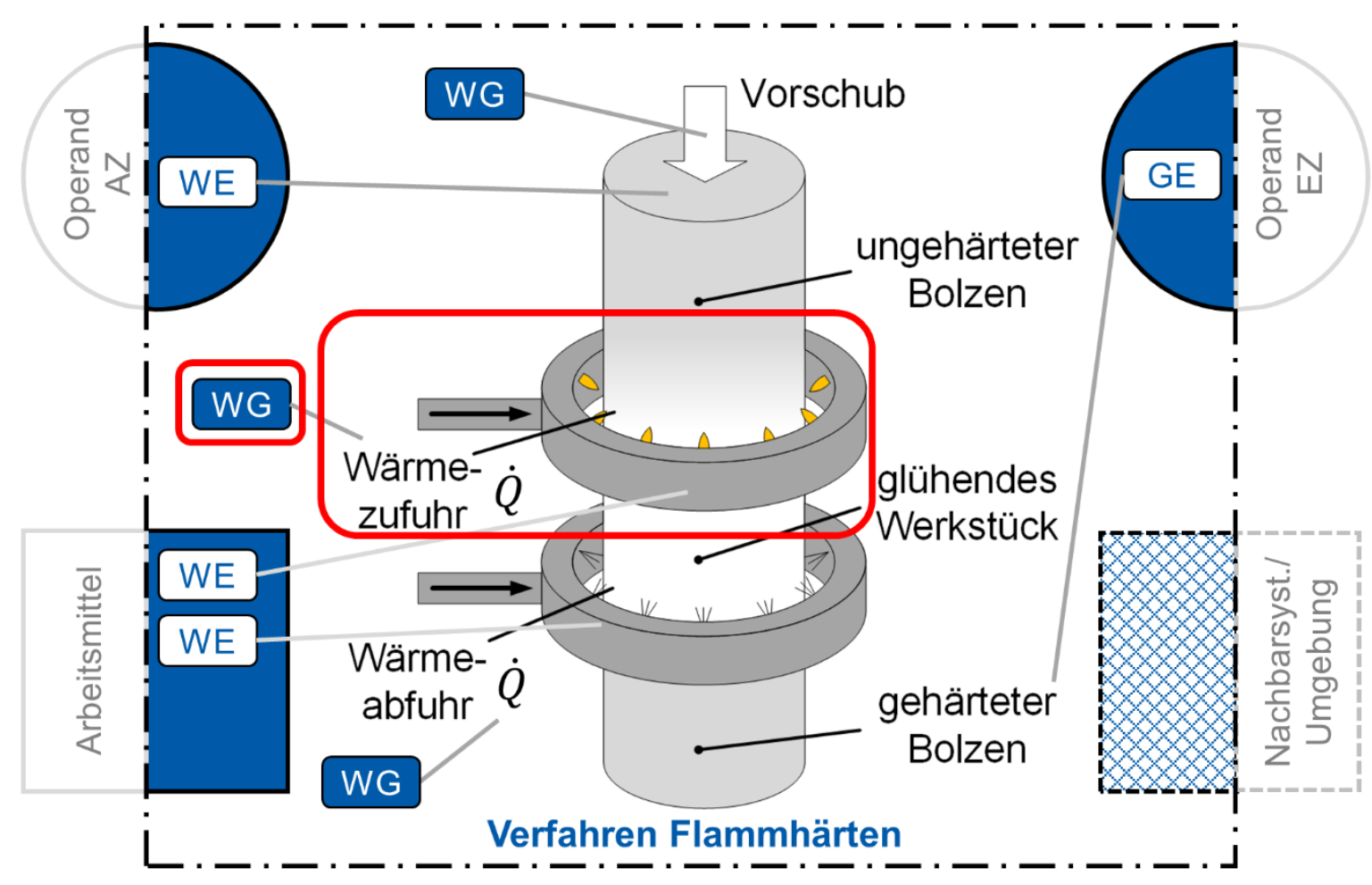

Abbildung 4-23: VP-Modell für das Flammhärten

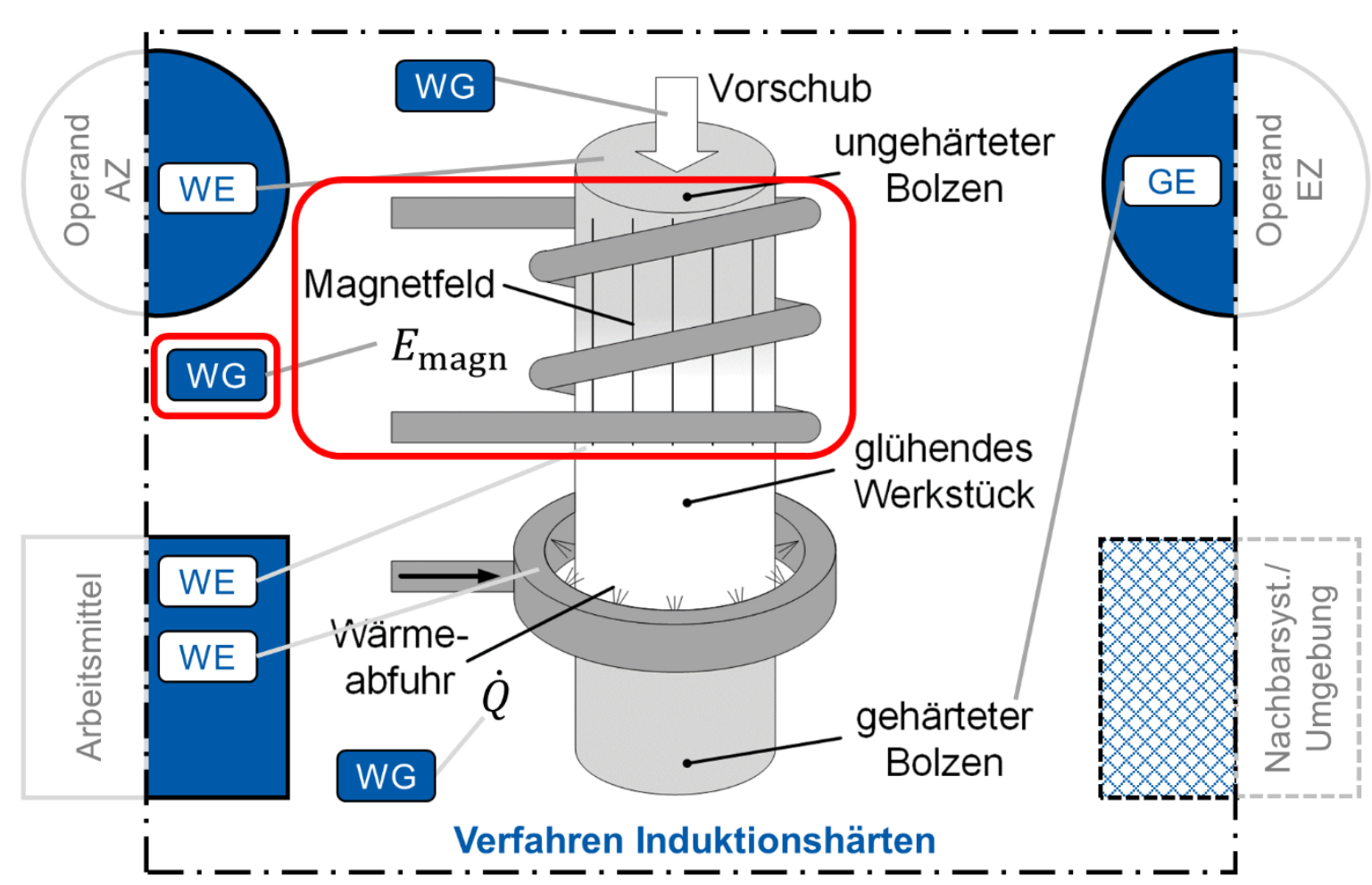

Abbildung 4-24: VP-Modell für das Induktionshärten

Für die weitere Konkretisierung wird das Verfahren Induktionshärten als Beispiel gewählt. Hierzu gilt es zu klären, wie die magnetische Energie, die als 
(Nenn-)Wirkgröße identifiziert wurde durch das Produkt (Arbeitsmittel des Einsatzprozesses) generiert werden kann. Nenn-Wirkgrößen (WG) aus dem Einsatzprozess fungieren im Produktkontext als Ausgangs-Funktionsgrößen. Sie sind somit das Bindeglied zwischen beiden Konkretisierungssträngen. Die Funktion des Produkts kann z. B. durch ein Wandeln einer elektrischen Energie $E_{\mathrm{el}}$ in die genannte magnetische Energie $E_{\text {magn }}$ realisiert werden. Abbildung 4-25 zeigt, wie diese Funktion über die weiteren Konkretisierungsebenen beschrieben werden kann. Mittels stromdurchflossener Leiter ist es möglich, auf Basis einer elektrischen Energie ein Magnetfeld zu erzeugen. Die Anordnung des Leiters als Wirkelement in Form einer Spule ermöglicht die Bündelung und Ausrichtung des Magnetfelds. Die Spulenform wird demnach durch die Konkretisierung der Produktfunktion bestimmt.

Die Produktgestalt des Arbeitsmittels ergibt sich aus den Festlegungen, die im Rahmen des Wirkprinzips bereits getroffen wurden und den Festlegungen, die in Bezug auf die verfahrensprinziprelevanten Wirkelemente (ringförmige Anordnung des Wirkelements) getätigt wurden. Die Spule sollte demnach nicht senkrecht zum Bolzen, sondern um diesen herum angeordnet werden, um eine möglichst gleichmäßige Erwärmung zu ermöglichen.

Funktion

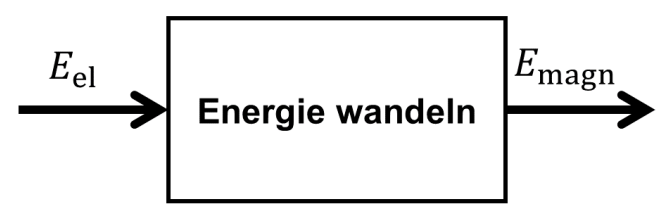

Physikalischer Effekt

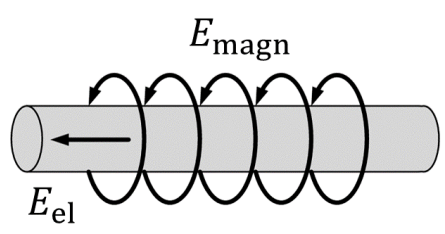

stromdurchflossener Leiter

Wirkprinzip

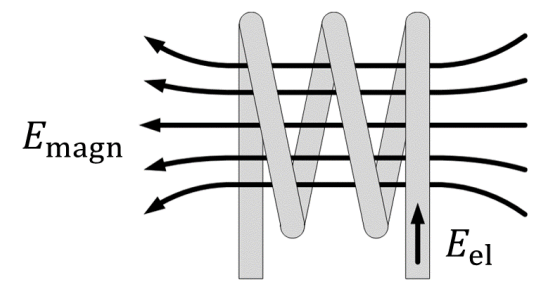

Anordnung des Leiters als Spule

Abbildung 4-25: Konkretisierung der Produktfunktion

Die modellübergreifende Konkretisierung des technischen Produkts durch die verschiedenen Ebenen der Einsatzprozess- und Produktmodellierung führt in letzter Konsequenz zu einer Produktgestalt, die sowohl der Produktfunktion als auch der 
angedachten Zustandsänderung im Einsatzprozess gerecht wird. Entscheidend für die Konkretisierung sind vor allem die Wirkgrößen aus dem Verfahrensprinzip, die das Bindeglied zwischen Prozess- und Produktkonkretisierung darstellen.

\subsection{Fazit - Abbildung fertigungstechnologischen Wissens}

Die konsequente Modellierung von Prozessen und Verfahren mittels Eigenschaften ermöglicht es, fertigungstechnologisches Produkt-Wissen in Modellen mit aufeinander aufbauenden Konkretisierungsgraden von der Zustandsänderung bis hin zum Verfahrensprinzip umfassend abzubilden (siehe Abschnitt 4.2). Eine besondere Bedeutung haben dabei die Verfahren, die das Wissen für die Konkretisierung des technischen Prozesses bereitstellen (siehe Abschnitte 4.1 und 4.3). Die eigenschaftsbasierte Modellierung technischer Prozesse stellt zudem die Kompatibilität zur Produktmodellierung sicher, wodurch eine einheitliche Modellwelt zur Produkt- und Prozessmodellierung geschaffen wird. Auf Basis dieser einheitlichen Modellwelt können das Produkt und seine Einsatzprozesse gleichermaßen konkretisiert und so eine Produktgestalt generiert werden, die der Produktfunktion und der angedachten Zustandsänderung im Einsatzprozess gerecht wird (siehe Abschnitt 4.3).

Das in Abschnitt 4.3 vorgestellte Vorgehen stellt einen ersten Ansatz für die gemeinsame Betrachtung des Produkts und seiner Lebenslaufprozesse im Rahmen der Produktentwicklung dar. Es wird jedoch auch deutlich, dass Fertigungsprozesse nicht direkt in dieses stringente Vorgehen eingeordnet werden können, da die Fertigung aufgrund ihrer vorgelagerten Stellung im Produktlebenslauf nur indirekt zum Kundennutzen beiträgt. Eine Konkretisierung von Fertigungsprozessen führt nicht direkt zur Steigerung des Kundennutzens. Folglich wird ein Ansatz benötigt, mittels dessen die Fertigung mit dem fertigungstechnologischen ProduktWissen auf eine andere Art und Weise in die Produktentwicklung integriert werden kann. 


\section{Ansatz zum Entwickeln fertigungsintegrierender Produktlösungen}

Es wird ein Ansatz vorgestellt, mittels dessen Fertigungspotenziale gezielt in Form fertigungsintegrierender Produktlösungen ausgeschöpft werden können. Dazu ist es notwendig, fertigungstechnologisches Produkt-Wissen in den Modellen der Produktentwicklung abzubilden, sodass eine frühzeitige Berücksichtigung und Integration des Wissens in den Produktkonkretisierungsprozess möglich wird. Aufbauend auf den Erkenntnissen der vorhergehenden Kapitel wird die grundlegende Idee des Ansatzes hergeleitet (siehe Abschnitt 5.1) und die Rolle des Antizipierens technischer Prozesse innerhalb dieses Ansatzes dargelegt (siehe Abschnitt 5.2). Auf dieser Grundlage werden das übergeordnete Vorgehen hinter dem Ansatz aufgezeigt (siehe Abschnitt 5.3) sowie im Rahmen einer umfassenden Methodik wesentliche Methoden und Werkzeuge angegeben, die insbesondere für die Entwicklung fertigungsintegrierender Produktlösungen prädestiniert sind (siehe Abschnitt 5.4). Vor allem die Design Pattern Matrix ${ }^{204}$ stellt ein Werkzeug dar, das den produkt- und prozessübergreifenden Ansatz unmittelbar im Rahmen der Lösungsfindung umsetzt (siehe Abschnitt 5.5).

\subsection{Idee - Modellübergreifende Verarbeitung fertigungstechnologischen Produkt-Wissens}

Bereits in den vorherigen Kapiteln dieser Arbeit wurde gezeigt, dass es aktuell keinen einheitlichen Weg gibt, fertigungstechnologisches Wissen im Rahmen eines stringenten Entwicklungsvorgehens zu verwenden. Das liegt vor allem darin begründet, dass Fertigungsprozesse anders als Einsatzprozesse nicht unmittelbar zur Bereitstellung eines Kundennutzens beitragen. Dennoch realisieren sie das Produkt und tragen damit wesentlich zu dessen Funktionserfüllung bei. Entsprechende Ansätze versuchen daher, die Einflüsse der Fertigung auf das Produkt gezielt zu erfassen und nutzbar zu machen. Im Rahmen des DfM wird meist auf speziell aufbereitete Richtlinien oder Hinweise zurückgegriffen, die vor allem in den späten Entwicklungsphasen Verwendung finden. Auch wenn sich Richtlinien und Hinweise als Hilfsmittel für den Produktentwickler etabliert haben, dienen diese dennoch häufig der Sicherstellung der Herstellbarkeit anstatt Fertigungspotenziale z. B. im Hinblick auf Leichtbau, Lebensdauer oder funktionaler Mehrwerte für die Konstruktion eines Produkts aufzuzeigen. In Vorgehensmodellen der Produktentwicklung, wie dem Axiomatic Design, werden Fertigungsprozessparameter

${ }^{204}$ Vgl. Roos et al. (2017a), S. 13. 
zwar in einem zusätzlichen Arbeitsschritt am Ende der Produktentwicklung unter Berücksichtigung der Einflüsse auf die Produktgestalt festgelegt. Dennoch bedingt auch dieses Vorgehen aufwendige Iterationen. Ähnlich wie die Ansätze des DfM vernachlässigt das Axiomatic Design zudem die Möglichkeit, dass Fertigungspotenziale bereits in den frühen Entwicklungsphasen zu gezielter Produktinnovation führen können.

Die in Abschnitt 4.2 vorgestellten Modelle zur Abbildung von Prozessen und Verfahren zeigen jedoch auch, dass es möglich ist, fertigungstechnologisches Produkt-Wissen so abzubilden, dass es grundsätzlich zu den Modellen der Produktentwicklung und damit zur Sichtweise des Produktentwicklers kompatibel ist. Darauf aufbauend soll eine Methodik erarbeitet werden, die die umfassende Aufarbeitung von fertigungstechnologischem Produkt-Wissen ermöglicht und dessen Übertragung von den Prozessmodellen hin zu den Produktmodellen methodisch unterstützt.

Zwei vielversprechende Anknüpfungspunkte liefern die GPPE und die produktionsintegrierende Entwicklungsmethodik nach GRAMLICH. Die GPPE basiert auf dem Kerngedanken, Lebenslaufprozesse bereits im Rahmen der Produktentwicklung zu antizipieren. Sie richtet den Blick des Produktentwicklers gezielt auf die Lebenslaufprozesse des Produkts, um Wissens zu diesen Prozessen während der Produktentwicklung umfassend zu berücksichtigen (siehe Abbildung 5-1 a).

a)

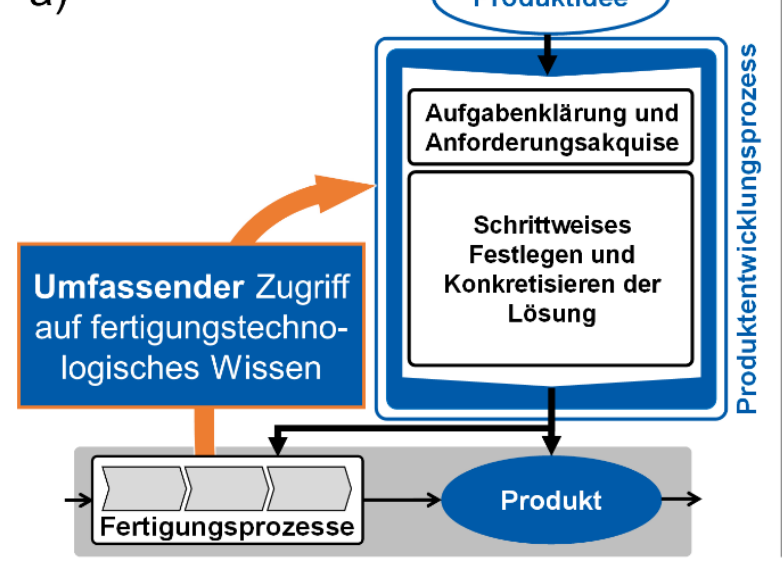

b)

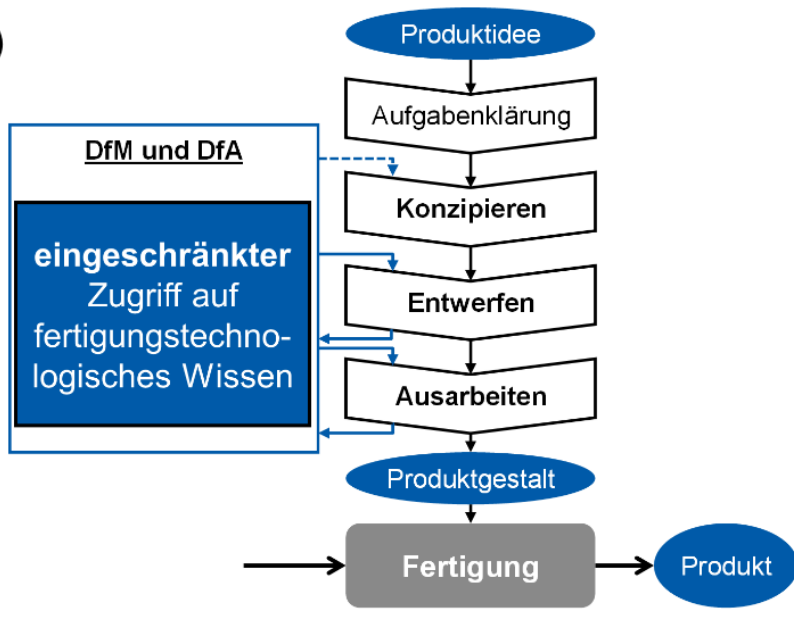

Abbildung 5-1: a) Umfassender vs. b) Eingeschränkter Wissenszugriff im Rahmen der Produktentwicklung

Damit unterscheidet sich die GPPE von Ansätzen, wie denen des DfM bzw. DfA (siehe Abbildung 5-1 b), die das Wissen bereits auf wesentliche Aussagen reduziert haben und es vor allem dazu verwenden, die Herstellbarkeit der Produkte 
sicherzustellen. Diese Ansätze ermöglichen daher nur einen eingeschränkten Zugriff auf das fertigungstechnologische Produkt-Wissen. Die produktionsintegrierende Entwicklungsmethodik nach GRAMLICH bietet darüber hinaus die Möglichkeit dieses Wissen auf Basis einer eigenschaftsbasierten Produkt- und Prozessmodellierung systematisch zu verarbeiten. Dies erfordert jedoch auch, das darin beschriebene Mapping von Eigenschaften weiterzuentwickeln und fertigungstechnologisches Produkt-Wissen gezielt mit weiterem Produkt-Wissen zu verknüpfen. Erst durch die systematische Verknüpfung von Eigenschaften über die Produktund Prozessmodelle hinweg kann die Lösungsfindung gezielt unterstützt werden.

\subsection{Antizipieren technischer Prozesse - Kernelement einer fertigungsintegrierenden Entwicklungsmethodik}

\subsubsection{Grundgedanke des Antizipierens technischer Prozesse}

Ein umfassender Zugriff auf fertigungstechnologisches Produkt-Wissen wird an verschiedenen Stellen im Produktentwicklungsprozess benötigt. So zeigt das bereits in Abschnitt 1.1 eingeführte Beispiel eines spaltprofilierten Führungsprofils für ein Leichtkransystem, in welchen Konkretisierungsschritten fertigungstechnologisches Wissen die technische Lösung beeinflusst (siehe Abbildung 5-2). Beim Gestalten des Führungsprofils ist auf eine möglichst biegesteife Ausführung des Profilquerschnitts zu achten. Vor allem im Bereich der Wälzkontaktflächen darf es zu keiner signifikanten Schrägstellung der Laufflächen kommen, da dies negative Auswirkungen auf das Laufverhalten der Wälzkörper und die Lebensdauer des Wälzkontakts haben kann. Zu einer solchen Schrägstellung kommt es auch, wenn sich das offene Profil über seiner Länge infolge Biegung zu stark absenkt und die Profilwände dadurch in Querrichtung beulen. Zu diesem Zweck können spaltgebogene Flansche ${ }^{205}$ als Versteifungsrippen eingebracht werden, die die Absenkung des Profils infolge Biegung reduzieren. Auch auf das Wirkprinzip der Produktlösung hat fertigungstechnologisches Produkt-Wissen einen Einfluss. Die spaltprofilierten Flansche eignen sich aufgrund ihrer werkstofflichen Eigenschaften als Wälzkontaktflächen. ${ }^{206}$ Aus diesem Grund wurden entsprechende Wirkflä-

\footnotetext{
205 Spaltbiegen ist im Wesentlichen eine Abwandlung des Spaltprofilierens. Dabei werden Flanschelemente nicht an der Bandkante, sondern an einer Biegekante des Blechhalbzeugs herausgearbeitet. (Vgl. Ringler, Groche (2008), S. 64 ff.)

${ }^{206}$ Vgl. Lommatzsch et al. (2011), S. 442.
} 
chen auf den spaltprofilierten Flanschen platziert (siehe Abbildung 5-2). Nicht zuletzt wird die Produktlösung durch fertigungstechnologisches Wissen auch im Hinblick auf die Erfüllung der Produktfunktion beeinflusst. Da spaltprofilierte Flansche eine signifikant geringere Kerbempfindlichkeit als das entsprechende Ausgangsmaterial aufweisen, wurde in das Führungsprofil die zusätzliche Funktionalität einer Profilverbindung integriert. Ausschlaggebend sind hierbei die Bohrlöcher, die der Verschraubung des Führungsprofils dienen. ${ }^{207}$

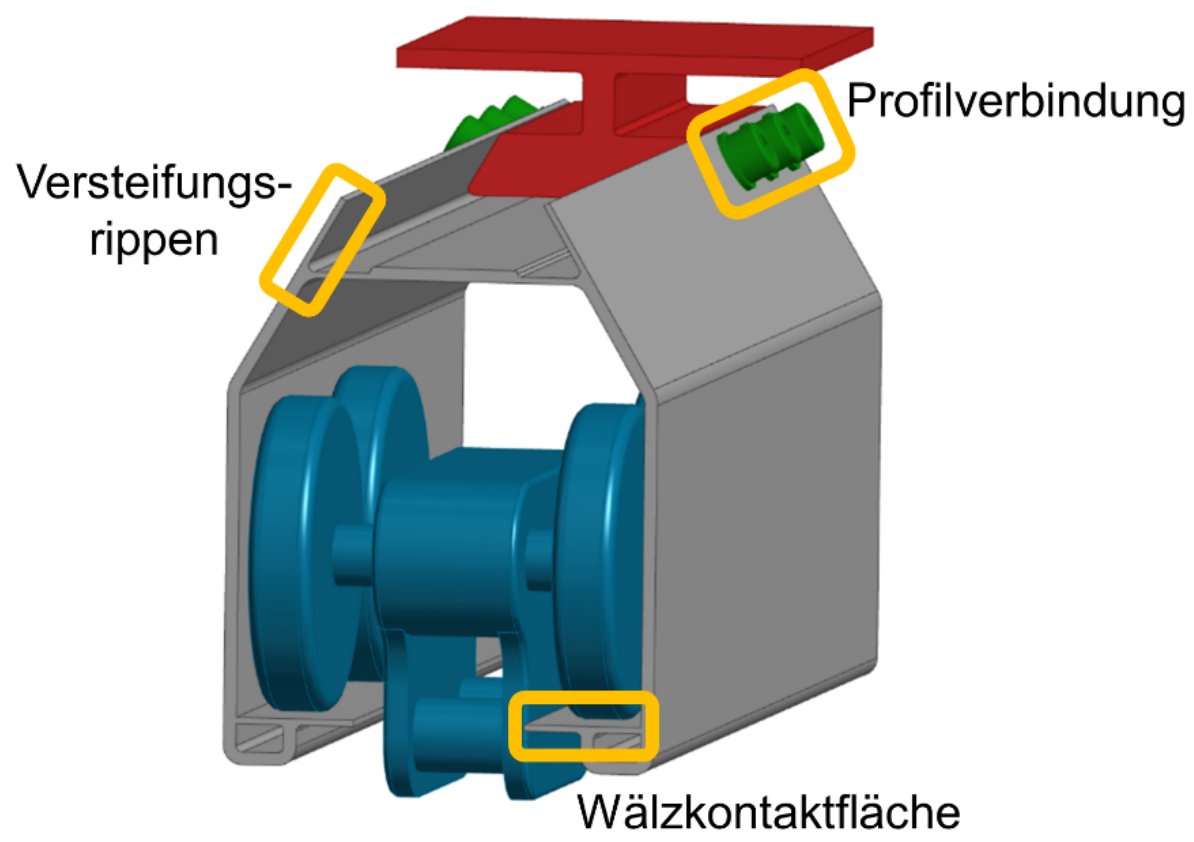

Abbildung 5-2: Einflüsse fertigungstechnologischen Wissens auf die Produktkonkretisierung am Beispiel eines spaltprofilierten Leichtkransystems ${ }^{208}$

Im Vorgehensmodell der Produktentwicklung wird ein Modellelement benötigt, das zu jedem Zeitpunkt der Entwicklung bzw. in allen Konkretisierungsschritten der Produktlösung den Rückgriff auf fertigungstechnologisches Wissen und damit die Verknüpfung zu Fertigungsprozessen ermöglicht. Ein entsprechendes Modellelement findet sich im Antizipieren technischer Prozesse der GPPE. Der Grundgedanke des Antizipierens im Rahmen der GPPE ist es, Lebenslaufprozesse bereits während der Produktentwicklung vorauszudenken, um so vor allem deren Wirkung auf die Umwelt abschätzen zu können. Das Antizipieren technischer Prozesse beruht auf einer Analyse dieser Lebenslaufprozesse. Erst dadurch können Aussagen

${ }^{207}$ Vgl. Gramlich et al. (2015).

208 In Anlehnung an Gramlich et al. (2015), S. 8. 
über die Umweltwirkungen der Prozesse und der daran beteiligten Produkte getroffen werden. Dieser Gedanke lässt sich auf die Entwicklung fertigungsintegrierender Produktlösungen übertragen, für die es notwendig ist, fertigungstechnologisches Produkt-Wissen möglichst umfassend der Produktentwicklung zur Verfügung zu stellen. Nur durch die Analyse der Fertigungsprozesse samt der innen zugrunde liegenden Verfahren ist es möglich, einen umfassenden Zugriff auf dieses Wissen zu gewährleisten. Auf diese Weise können nicht nur die Auswirkungen konstruktiver Entscheidungen auf die Herstellbarkeit des Produkts herausgearbeitet, sondern auch Möglichkeiten erschlossen werden, die sich dem Entwickler in Bezug auf zusätzliche Ansätze zur Funktionserfüllung bieten.

Das Antizipieren von Lebenslaufprozessen geht immer mit einem vorherigen „Beeinflussen" dieser Prozesse einher. So können z. B. Fertigungsverfahren, Umgebungsbedingungen, etc. bereits während des Konkretisierens der technischen Lösung festgelegt werden. Das Antizipieren im Sinne einer Prozessanalyse wird vorgenommen, um die Auswirkungen dieser vorherigen konstruktiven Festlegungen auf Lebenslaufprozesse bereits während des Konkretisierens der technischen Lösung vorauszudenken. Daraus können später Schlussfolgerungen für die Produktentwicklung gezogen werden. Antizipieren und Beeinflussen bilden damit eine untrennbare Einheit.

Auf Basis der in Kapitel 4 erarbeiteten Modelle kann fertigungstechnologisches Produkt-Wissen mittels Eigenschaften und deren Relationen einheitlich abgebildet werden. Das Zurückgreifen auf diese eigenschaftsbasierten Modelle im Rahmen des Antizipierens technischer Prozesse stellt sicher, dass die aus der Prozessanalyse gewonnenen Eigenschaften und Eigenschaftsrelationen im Rahmen der Produktentwicklung verwendet werden können. Das Antizipieren ist demnach ein zielgerichtetes, systematisches Ermitteln von Eigenschaften ${ }^{209}$ im Kontext technischer Prozesse, das in Form modellbasierter Analysen ausgehend von den Eigenschaften, die während der Lösungskonkretisierung auf dem jeweiligen Konkretisierungsniveau festgelegt wurden, durchgeführt wird. Daraus leiten sich Entscheidungen ab, die die weitere Konkretisierung der technischen Lösung im Rahmen des Produktkonkretisierungsprozesses betreffen. Das Antizipieren definiert sich daher wie folgt:

${ }^{209}$ Das können Soll-, Nenn- oder Ist-Eigenschaften sein. 
Das Antizipieren technischer Prozesse des Produktlebenslaufs ist eine zielgerichtete, auf das jeweilige Konkretisierungsniveau angepasste modellbasierte Prozessanalyse ausgehend von bereits getätigten konstruktiven Festlegungen während des Produktentwicklungsprozesses.

Die Wahl der Modelle, die der Analyse zugrunde liegen, richtet sich grundsätzlich nach dem Analysezweck. Dieser kann sehr unterschiedlicher Natur sein. Der Zweck des Antizipierens technischer Prozesse resultiert meist aus Fragen, die während der eigentlichen Entwicklungstätigkeit auftauchen (z. B. Umweltwirkungen feststellen, Fertigungspotenziale ausschöpfen oder Montage sicherstellen).

\subsubsection{Antizipieren von Fertigungsprozessen}

Insbesondere die Modellierung des Verfahrensprinzips ermöglicht die umfassende Abbildung fertigungstechnologischen Produkt-Wissens in Form von Eigenschaften und deren Relationen. Auf diese Weise liegt es in formalisierter Form vor und kann in nahezu allen kompatiblen (d. h. eigenschaftsbasierten) Modellen der Produktentwicklung weiterverwendet werden.

Es sind vor allem die (fertigungs-)verfahrensinduzierten Gestaltelemente und ihre Eigenschaften, die im Rahmen des Antizipierens von Fertigungsprozessen erschlossen werden müssen. Bereits GRAMLICH attestiert den (fertigungs-)verfahrensinduzierten Eigenschaften eine hohe Bedeutung im Rahmen der Produktentwicklung. Sie umfassen die wesentlichen Eigenschaften der realisierbaren Produkte und charakterisieren somit die zugrunde liegenden Verfahren. ${ }^{210}$ Für das Beispiel des Verfahrens Spaltbiegen ${ }^{211}$ zeigt Abbildung 5-3, wie die verfahrensinduzierten Eigenschaften spaltgebogener Flansche mittels des VP-Modells identifiziert werden können. Neben der dargestellten Flanschlänge $l$ werden die spaltgebogenen Flansche durch weitere verfahrensinduzierte Eigenschaften charakterisiert, die hier jedoch nicht näher betrachtet werden. Mittels des VP-Modells können in gleicher Weise die verfahrensinduzierten Gestaltelemente aller denkbaren Verfahren ermittelt werden. Abbildung 5-4 zeigt auszugsweise das Beispielverfahren Bohren, dem ein spaltprofiliertes Halbzeug zugrunde liegt. Darin ist vor allem das Gestaltelement Bohrloch mit seinem Durchmesser $d_{B}$ von grundsätzlichem Interesse für den Entwickler und die weitere die Auslegung seiner Produkte.

${ }^{210} \mathrm{Vgl}$. Gramlich (2013), S. $90 \mathrm{ff}$.

${ }^{211}$ Vgl. Ringler, Groche (2008), S. 64 f. 


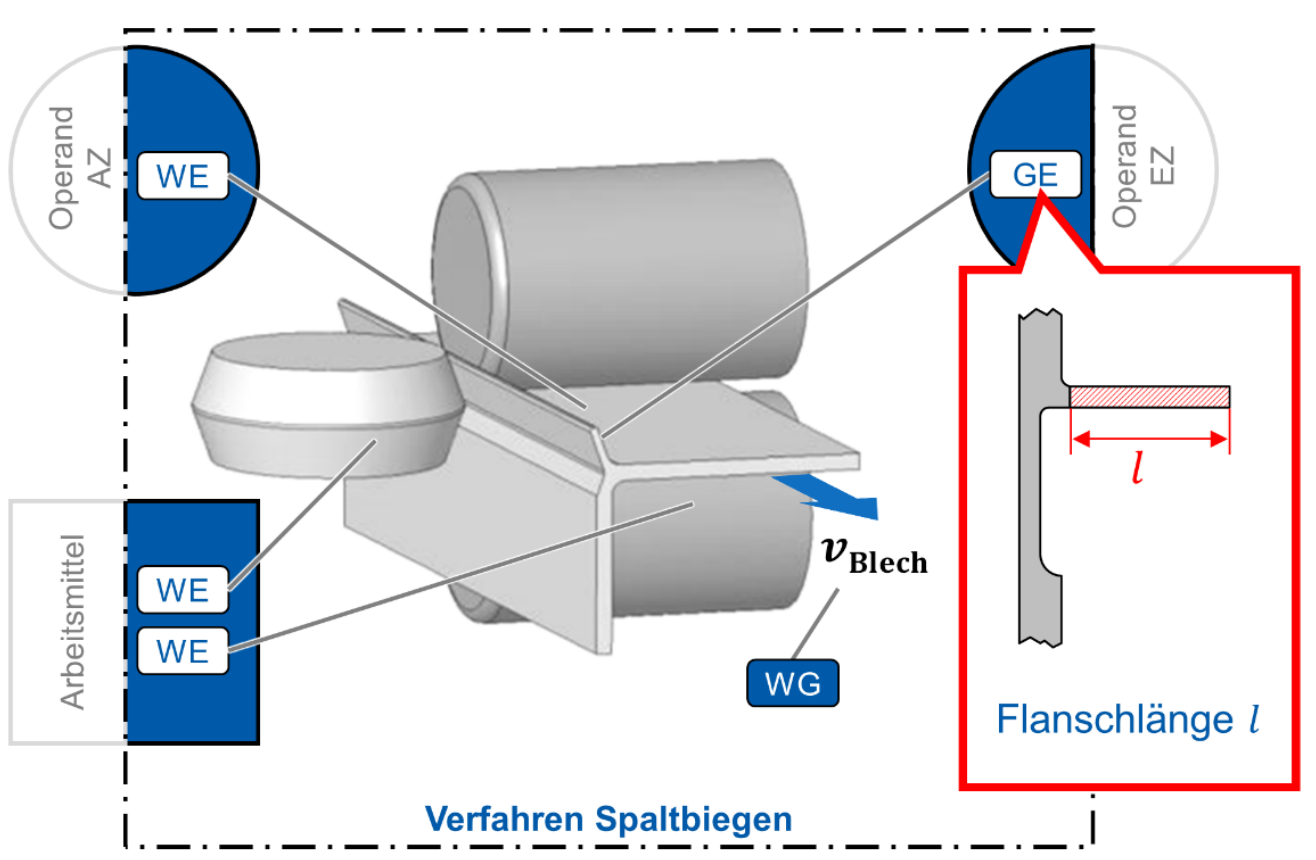

Abbildung 5-3: Antizipieren verfahrensinduzierter Eigenschaften und Gestaltelemente am Beispiel des Spaltbiegens

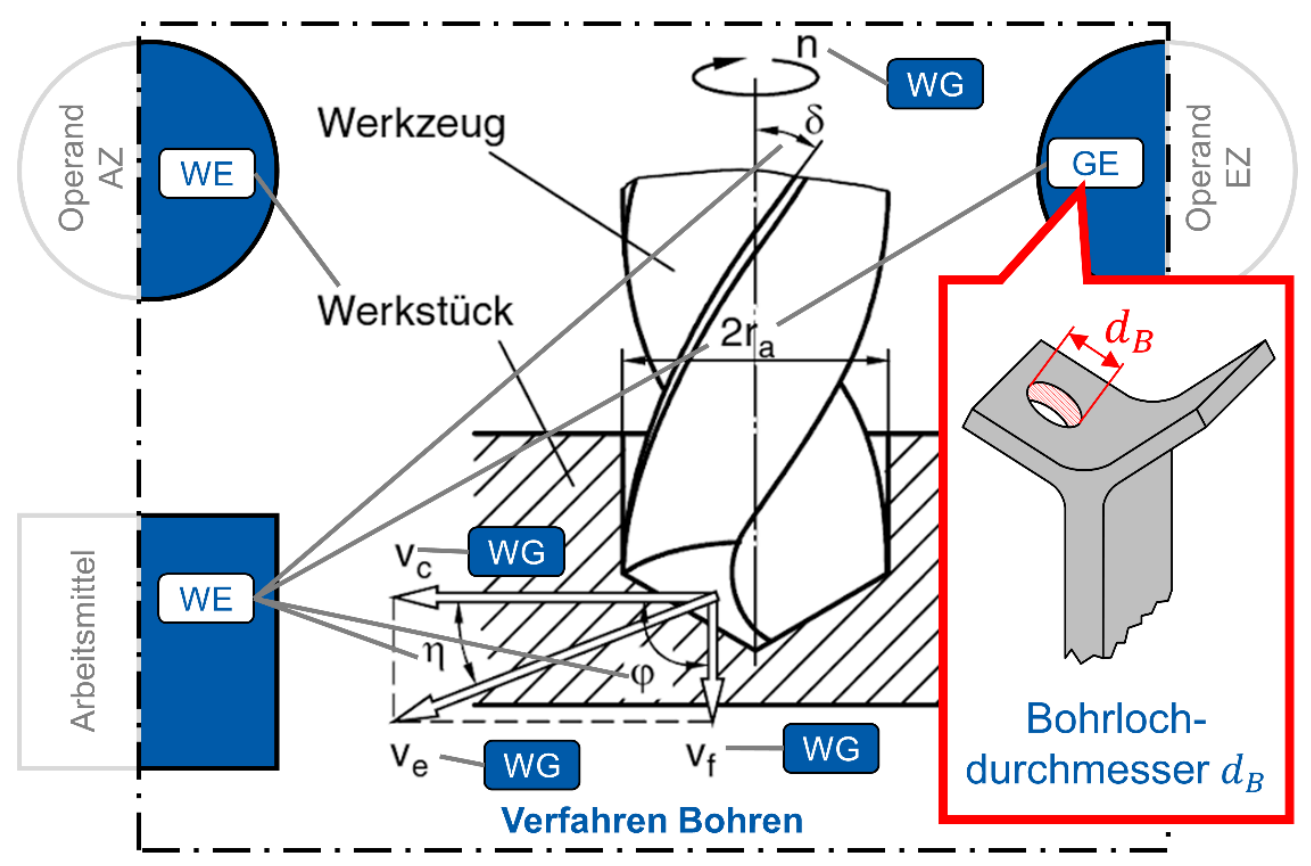

Abbildung 5-4: Antizipieren verfahrensinduzierter Eigenschaften und Gestaltelemente am Beispiel des Bohrens von Löchern in spaltprofilierte Flansche ${ }^{212}$

Abbildung 5-5 zeigt das schematische Vorgehen, das durch Verwendung des VPModells die systematische Analyse des entsprechenden Fertigungsverfahrens ermöglicht.

212 In Auszügen aus Denkena, Tönshoff (2011), S. 11. 

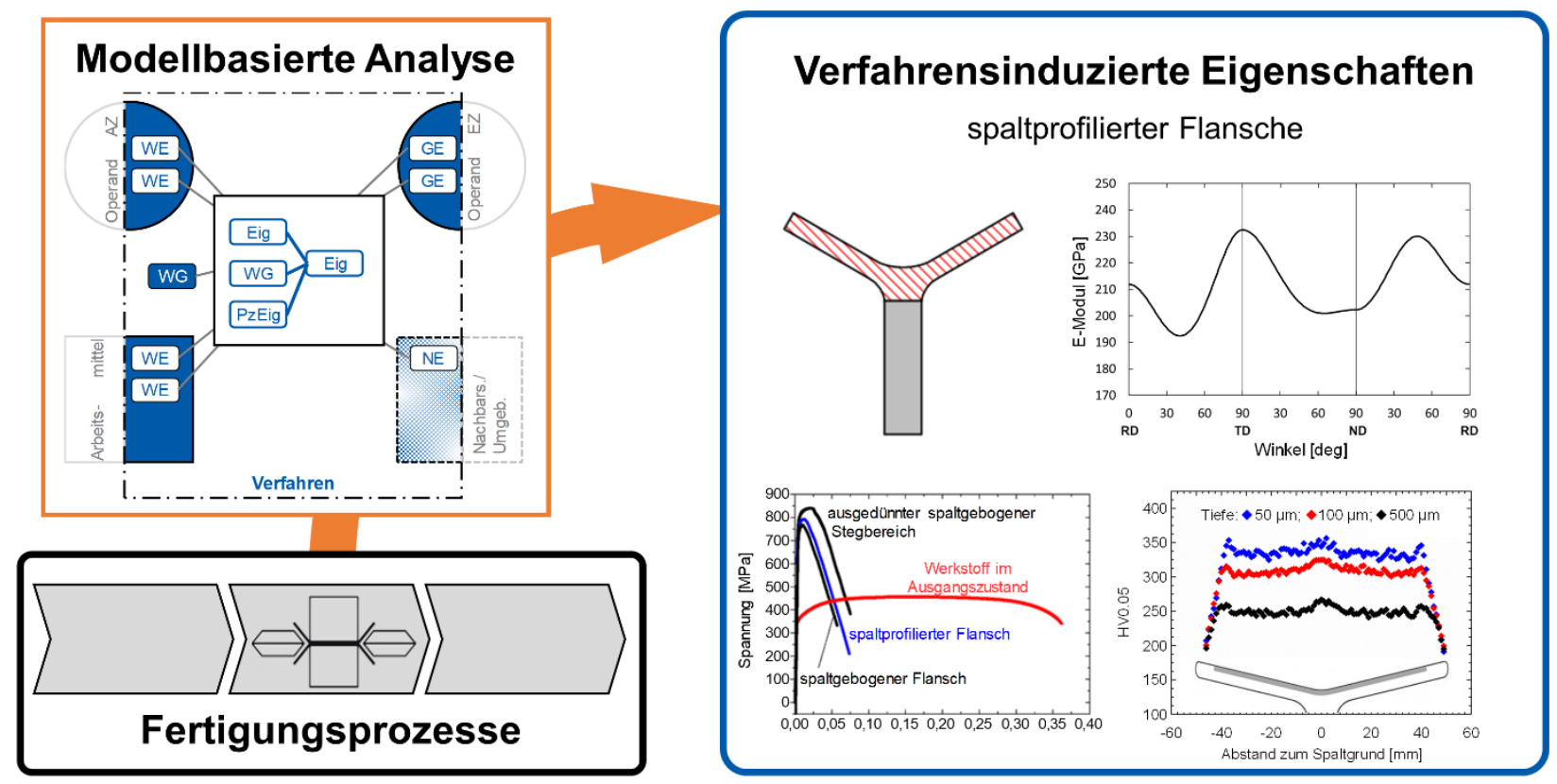

Abbildung 5-5: Antizipieren von Fertigungsprozessen am Beispiel des
Spaltprofilierens ${ }^{213}$

Erst durch die Kenntnis der verfahrensinduzierten Eigenschaften kann der Entwickler weitere Möglichkeiten ausloten, die sich für die Umsetzung innovativer Produktlösungen ergeben. Die Wirkzusammenhänge mit den verfahrensprinziprelevanten Wirkelementen können dennoch nicht vernachlässigt werden. Erst durch sie kann der Entwickler Stellhebel im Prozess identifizieren, mittels derer er auch ohne umfassendes Know-how über Fertigungsverfahren die Ausprägung der verfahrensinduzierten Eigenschaften beeinflussen kann (z. B. Wahl des Halbzeugs).

Das Antizipieren von Fertigungsprozessen unter Verwendung von Prozessmodellen zeichnet sich durch seinen checklistenähnlichen Charakter aus. Dabei geht es vor allem darum, aus den Verfahren die für die Produktentwicklung relevanten Eigenschaften und Eigenschaftsrelationen zu identifizieren. Gleiches gilt auch beim Antizipieren von Umweltwirkungen, dem ursprünglichen Zweck des Antizipierens im Rahmen der GPPE. Dabei dient das Antizipieren technischer Prozesse in erster Linie der Identifikation relevanter Materialen und Energien, die im Produktlebenslauf verarbeitet werden. Die eigentlichen Umweltwirkungen finden jedoch in einem separaten Auswertungsschritt statt, der durch geeignete Methoden und Werkzeuge unterstützt wird. ${ }^{214}$ Das führt zu der Schlussfolgerung, dass im Rahmen ei-

213 In Auszügen aus Ahmels et al. (2017), S. 108, 111; Niehuesbernd et al. (2013).

${ }^{214} \mathrm{Vgl}$. DIN EN ISO 14040 (2009). 
ner fertigungsintegrierenden Entwicklungsmethodik neben dem frühzeitigen Antizipieren von Fertigungsprozessen weitere Schritte notwendig sind, um mögliche Auswirkungen der Fertigung auf die Konkretisierung des Produkts zu erarbeiten. So können Fertigungspotenziale umfassend ausgeschöpft werden.

\subsection{Vorgehensmodell zum Entwickeln fertigungsintegrierender Produktlösungen}

Das Antizipieren von Fertigungsprozessen kann zu verschiedenen Zeitpunkten während des Entwicklungsprozesses durchgeführt werden. D. h. ein Initiieren des Vorgehens ist auf jeder Produktkonkretisierungsebene möglich. Darüber hinaus bedingt das Antizipieren von Fertigungsprozessen bestimmte vor- und nachgelagerte Vorgehensschritte, ohne die es nicht in ein übergeordnetes Vorgehensmodell eingebunden werden kann. Bereits in der GPPE wurde der Schritt des Beeinflussens angesprochen und durch zwei Grundgedanken adressiert. Zum einen werden durch konstruktive Festlegungen am technischen Produkt auch die Lebenslaufprozesse zu einem bestimmten Grad festgelegt. Dieser Aspekt wird im Rahmen einer fertigungsintegrierenden Entwicklungsmethodik dadurch abgebildet, dass die Produktentwicklung nicht nur die Produktgestalt festlegt, sondern auch die Fertigungsprozesse des Produktlebenslaufs. Dieses Festlegen wird durch die schwarzen Pfeile in Abbildung 5-6 repräsentiert, die vom Produktentwicklungsprozess auf die Fertigungsprozesse und das Produkt deuten. Zum anderen umfasst das Beeinflussen auch ein Vorbereiten der eigentlichen Prozessanalyse. So müssen bereits vorhandene konstruktive Festlegungen in die Prozessmodellierung und die nachfolgende Analyse einbezogen werden, um daraus verwertbare Aussagen zu erhalten (siehe blauer Pfeil mit der Beschriftung „Beeinflussen“ in Abbildung 5-6). Die mittels des Antizipierens identifizierten und für die Produktentwicklung relevanten Eigenschaften und Eigenschaftsrelationen stellen selbst nur einen begrenzten Mehrwert für die Produktkonkretisierung dar, solange nicht klar ist, wie sie für das Produkt genutzt werden können. Sie sind das Rohmaterial für die Suche nach fertigungsintegrierenden Lösungen. Ein nachgelagerter Vorgehensschritt ist notwendig, in dem diese Eigenschaften und Eigenschaftsrelationen mit Blick auf die während der Produktkonkretisierung verwendeten Modelle aufgearbeitet werden (siehe blauer Pfeil mit der Beschriftung „Integrieren“ in Abbildung 5-6). 


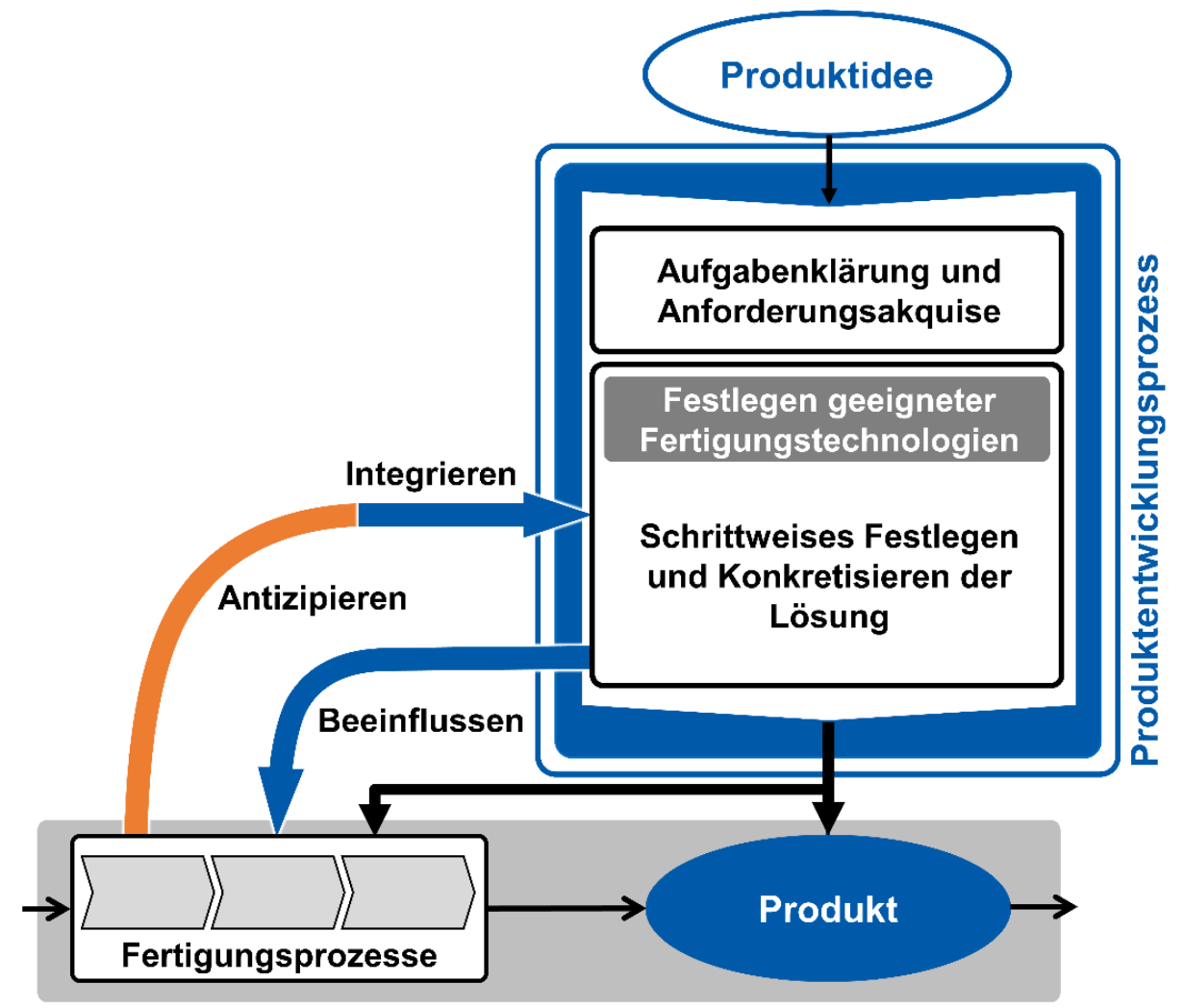

Abbildung 5-6: Vorgehensmodell einer fertigungsintegrierenden Entwicklungsmethodik auf Basis des Antizipierens technischer Prozesse

Sowohl das Beeinflussen als auch das Integrieren sind Vorgehensschritte, die durch den Produktentwickler vorgenommen werden müssen. Sie bedingen die Auseinandersetzung mit der Produktkonkretisierung. Das Antizipieren von Fertigungsprozessen ist hingegen ein Schritt, der sowohl die Sicht der Produktentwicklung als auch die der Fertigung auf Basis einer einheitlichen Modellwelt kombiniert. Dieser Schritt sollte in enger Absprache von Fertiger und Produktentwickler bzw. im Rahmen eines Projektteams durchgeführt werden.

Für das Entwickeln fertigungsintegrierender Produktlösungen ist stets auch das vorherige Festlegen geeigneter Fertigungstechnologien notwendig. Erst danach kann im Rahmen der gewählten Verfahren fertigungstechnologisches Wissen identifiziert und für die Produktentwicklung aufgearbeitet werden (siehe Abbildung 5-6). Mit der produktionsintegrierenden Entwicklungsmethodik nach GRAMLICH und dem Integrated Algorithm-Based Product and Process Development existieren bereits Ansätze, die darlegen, wie die Festlegung geeigneter Fertigungstechnologien durch ein frühzeitiges Antizipieren von Fertigungsprozessen unterstützt werden kann. ${ }^{215}$

${ }^{215}$ Vgl. Gramlich (2013), S. 132 ff.; Lüthen et al. (2017), S. 159 ff. 


\subsection{Fertigungsintegrierende Entwicklungsmethodik}

Das in Abbildung 5-6 vorgestellte Vorgehensmodell bildet die Basis für eine detaillierte Methodik, die eine Operationalisierung des eigentlichen Ansatzes zum Entwickeln fertigungsintegrierender Produktlösungen darstellt. Dazu werden die Vorgehensschritte Beeinflussen, Antizipieren und Integrieren in einzelne Arbeitsschritte unterteilt und innen geeignete Hilfsmittel (Methoden und Werkzeuge) zugeordnet (siehe Abbildung 5-7). Ausgangspunkt für die Anwendung der Methodik ist stets ein konkretes Entwicklungsprojekt. Dementsprechend liegt der Anwendung der Methodik ein projektspezifisches Ziel zugrunde (siehe Arbeitsschritt 1 in Abbildung 5-7). Für die Entwicklung fertigungsintegrierender Produktlösungen am Beispiel des vorgestellten Leichtkransystems lautet dieses Ziel: „Aufbereiten fertigungstechnologischen Wissens, um Fertigungspotenziale für die Realisierung des Leichtkransystems zu erschließen“. Die Zielformulierung muss stets dem Zweck des Antizipierens entsprechen bzw. den Nutzen für die Entwicklung adressieren.

\begin{tabular}{|c|c|c|c|}
\hline \multirow{2}{*}{ 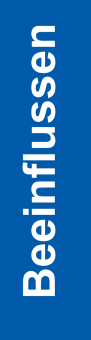 } & \multirow[b]{2}{*}{2} & \multicolumn{2}{|l|}{$\begin{array}{l}\text { Ziel festlegen } \\
\text { Projektspezifisches Ziel der Untersuchung formulieren }\end{array}$} \\
\hline & & $\begin{array}{l}\text { Betrachtungsrahmen festlegen } \\
\text { Definition projektspezifisch zu untersuchender Prozesse } \\
\text { unter Berücksichtigung vorheriger konstruktiver } \\
\text { Festlegungen }\end{array}$ & $\begin{array}{l}\text { Hilfsmittel: } \\
\text { Produktmodelle }\end{array}$ \\
\hline & 3 & $\begin{array}{l}\text { Modellauswahl } \\
\text { Auswahl geeigneter Prozessmodelle }\end{array}$ & Hilfsmittel: \\
\hline & 4 & $\begin{array}{l}\text { Prozessmodellbasierte Analyse } \\
\text { Anwenden der Prozessmodelle, um Wissen aus den } \\
\text { Prozessen des Produktlebenslaufs abzubilden }\end{array}$ & weitere Prozessmodelle \\
\hline (1) & 5 & $\begin{array}{l}\text { Aufbereiten der Analyseergebnisse } \\
\text { Allgemeingültige Aufbereitung der Analyseergebnisse } \\
\text { durch Verknüpfen verfahrensinduzierter Eigenschaften mit } \\
\text { Produkt-Wissen }\end{array}$ & $\begin{array}{l}\text { Hilfsmittel: } \\
\text { DPM, Produkt- und } \\
\text { Prozessmodelle }\end{array}$ \\
\hline 토 & 6 & $\begin{array}{l}\text { Maßnahmen ergreifen und Auswerten } \\
\text { Projektspezifische Verarbeitung der aufbereiteten } \\
\text { Analyseergebnisse }\end{array}$ & $\begin{array}{c}\text { Hilfsmittel: } \\
\text { DPM, Punktbewertung }\end{array}$ \\
\hline
\end{tabular}

Abbildung 5-7: Detailliertes Vorgehen für das Beeinflussen, Antizipieren und Integrieren im Rahmen einer fertigungsintegrierenden Entwicklungsmethodik 
Die systematische Erarbeitung fertigungstechnologischer Produktlösungen erfordert es, die während der Produktkonkretisierung getroffenen Festlegungen bereits frühzeitig in das vorgestellte Vorgehen einzubeziehen. Für den vorbereitenden Arbeitsschritt "Betrachtungsrahmen festlegen" (siehe Arbeitsschritt 2 in Abbildung 5-7) ist es daher unabdingbar, diese konstruktiven Festlegungen herauszuarbeiten. Für das Leichtkransystem sind $u$. a. die bereits festgelegten Fertigungsverfahren Spaltprofilieren und Spaltbiegen zu berücksichtigen. Diese Festlegung verringert insbesondere die Komplexität des Antizipierens deutlich. Darüber hinaus können auf Basis vorhandener Produktmodelle bereits die grundlegenden Funktionen des Leichtkransystems identifiziert werden. Diese umfassen die Aufnahme von Lasten, das Führen des Schlittens innerhalb des Führungsprofils und die Aufhängung des Führungsprofils. Als Tragstruktur, die eine Biegebelastung aufgrund der angehängten Last erfährt, muss die spätere Lösung eine möglichst hohe Biegesteifigkeit aufweisen. Die Biegesteifigkeit kann insbesondere durch die Querschnittsgeometrie der Tragstruktur beeinflusst werden. Abbildung 5-8 a) zeigt den entsprechenden Funktionsträger für einen Biegebalken, dessen Biegesteifigkeit insbesondere durch die Veränderung seiner Höhe geändert werden kann. Die Kenntnis der bereits identifizierten Funktionsträger ist im weiteren Verlauf des Vorgehens von essenzieller Bedeutung, um die Produktlösung im Einklang mit dem fertigungstechnologischen Produkt-Wissen zu erarbeiten.

Im Rahmen des Antizipierens der Fertigungsprozesse bzw. derer Verfahren gilt es, Prozessmodelle zu verwenden, die mit den Produktmodellen während des Konkretisierens kompatibel sind. Nur so können die für den Produktentwickler relevanten Eigenschaften herausgearbeitet werden (siehe Arbeitsschritt 3 in Abbildung 5-7). In Abschnitt 5.2.2 wurde am Beispiel des Spaltbiegens gezeigt, wie unter Verwendung des VP-Modells die relevanten verfahrensinduzierten Eigenschaften und Gestaltelemente identifiziert werden können (siehe Arbeitsschritt 4 in Abbildung 5-7). Abbildung 5-8 b) stellt dies dem Ergebnis aus Arbeitsschritt 2 gegenüber. 
a)

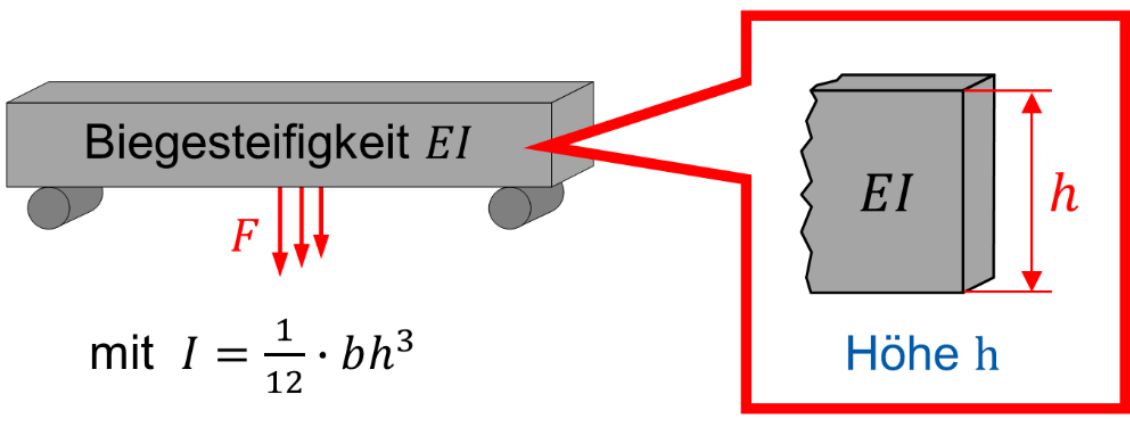

b)

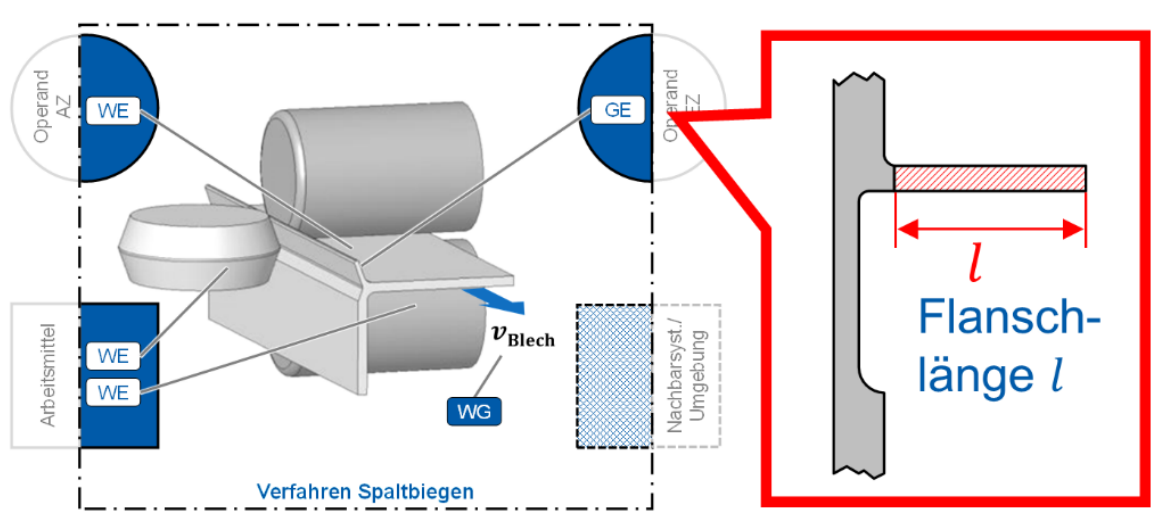

Abbildung 5-8: a) Funktionsträger der Tragstruktur eines Leichtkransystems; b) antizipiertes Gestaltelement für das Verfahren Spaltbiegen

Die systematische Erarbeitung fertigungsintegrierender Produktlösungen beruht vor allem auf der Verknüpfung von fertigungstechnologischem Produkt-Wissen mit weiterem Produkt-Wissen auf der Grundlage aufeinander abgestimmter Produktund Prozessmodelle. Dazu müssen die identifizierten verfahrensinduzierten Eigenschaften mit Eigenschaften aus den Produktmodellen verknüpft werden. Am Beispiel des Leichtkransystems sind dies $u$. a. die Eigenschaften der einzelnen Funktionsträger, die das Produkt auf Basis geeigneter Produktmodelle genauer beschreiben. Dazu werden im Arbeitsschritt 5 (siehe Abbildung 5-7) „Aufbereiten der Analyseergebnisse" Methoden und Tools benötigt, die die modellbasierte Verknüpfung dieser Eigenschaften unterstützen. Eine umfassende Unterstützung liefert z. B. die Design Pattern Matrix (DPM), die in Abschnitt 5.5 beschrieben wird. Im Arbeitsschritt 6 (siehe Abbildung 5-7) werden darauf aufbauend projektspezifische Maßnahmen abgeleitet, die auf verschiedenen Konkretisierungsebenen des Produkts angewendet werden können. Dieser Arbeitsschritt kann u. a. durch Methoden zum Beurteilen von Lösungsalternativen, wie beispielweise eine Punktbewertung ${ }^{216}$ unterstützt werden. Die Auswirkungen auf die Produktlösungen sind 
von Projekt zu Projekt sehr unterschiedlich, wie später anhand der in Kapitel 6 dargelegten Beispiele verdeutlicht wird. Eine Übersicht über die Arbeitsschritte der Methodik und deren Ergebnisse ist in Anhang $D$ zu finden.

Die dargelegte Methodik zeichnet sich durch zwei zentrale Elemente aus. Zum einen ermöglichen die im Rahmen des Antizipierens durchgeführten Prozessanalysen die direkte Verarbeitung fertigungstechnologischen Produkt-Wissens mit Blick auf die Modellwelt der Produktentwicklung (Schritte 3 und 4). Zum anderen wird dieses Wissen für die Produktentwicklung nutzbar, indem geeignete Eigenschaften in der Modellwelt der Produktentwicklung verknüpft werden (Schritt 5). Ohne diese beiden Elemente kann der systematische Wissenstransfer bzw. die methodische Nutzbarmachung fertigungstechnologischen Produkt-Wissens in der Produktentwicklung nur schwer gelingen. Ein Werkzeug, das diese Verknüpfung unterstützt, wird im folgenden Abschnitt dargelegt.

\subsection{Design Pattern Matrix - Werkzeug zur Entwicklung fertigungsintegrierender Produktlösungen}

\subsubsection{Fertigungsintegrierende Design Patterns}

Auf Basis der einheitlichen Beschreibung technischer Prozesse und Produkte mittels Eigenschaften können wiederkehrende Eigenschaftsmuster identifiziert werden, die als Lösungselemente ${ }^{217}$ im Entwicklungsprozess genutzt werden können. ${ }^{218}$ Bereits WEBER formuliert im Rahmen des Characteristics-Properties Modelling (CPM $)^{219}$ sogenannte Solution Patterns, auf deren Grundlage er Konstruktionswissen als wiederkehrende Kombinationen aus Characteristics, Properties und deren Relationen beschreibt. ${ }^{220}$ Diese können zur gezielten Unterstützung von Analyse- und Syntheseschritten im Rahmen des Property-Driven-Development (PDD) eingesetzt werden. ${ }^{221}$ In Anknüpfung an die Solution Patterns des PDD

217 Lösungselemente stellen im Rahmen dieser Arbeit noch keine vollwertigen Lösungen eines technischen Problems dar. Sie sind vielmehr allgemeingültige Bestandteile potenzieller technischer Lösungen. Im Rahmen der projektspezifischen Lösungsfindung können die Lösungselemente für die Generierung von Varianten genutzt werden, aus denen die eigentliche technische Lösung hervorgeht.

${ }^{218} \mathrm{Vgl}$. Wagner et al. (2016), S. 741.

${ }^{219}$ Vgl. Weber (2012), S. $31 \mathrm{ff}$.

${ }^{220}$ Vgl. Weber (2012), S. $42 \mathrm{f}$.

${ }^{221}$ Vgl. Weber, Werner (2000), S. 130. 
können auch im Kontext des dieser Arbeit zugrunde liegenden Eigenschaftsbegriffs Eigenschaftsmuster identifiziert werden, die gezielt zur Unterstützung der fertigungsintegrierenden Entwicklungsmethodik verwendet werden sollen.

Inwiefern ein Muster aus Eigenschaften und deren Relationen tatsächlich als Lösungselement in einem konkreten Entwicklungsprojekt verwendet werden kann, wird von Produktmodellen bestimmt. Produktmodelle, wie z. B. ein Modell der Produktfunktion, definieren daher die Relevanz eines Eigenschaftsmusters für die Lösungsfindung. Die Kombination aus Eigenschaftsmuster und Produktmodell wird als Design Pattern bezeichnet. Die Eigenschaften und deren Relationen repräsentieren darin das formalisierte Wissen über das Lösungselement. Erst durch dieses Wissen ist es möglich, die Design Patterns im Rahmen der Produktentwicklung zu verwenden.

Dem grundlegenden Aufbau von Design Patterns folgend können unter Einbeziehung von fertigungstechnologischem Wissen sogenannte fertigungsintegrierende Design Patterns erarbeitet werden. Durch die Verknüpfung von verfahrensinduzierten Eigenschaften mit Eigenschaften, die die Produktfunktion beschreiben ${ }^{222}$, können Eigenschaftsmuster identifiziert werden, die unter Angabe entsprechender Produktmodelle als Lösungselemente im Kontext einer fertigungsintegrierenden Entwicklungsmethodik verwendet werden können. Diese Lösungselemente stellen fertigungsintegrierende Design Patterns dar. Unter Berücksichtigung der Schlüsselelemente von Design Patterns kann die folgende Definition fertigungsintegrierender Design Patterns gegeben werden.

Fertigungsintegrierende Design Patterns sind allgemeingültige Lösungselemente, die auf der Verknüpfung von Eigenschaften zur Produktfunktion und Eigenschaften zu einem Fertigungsverfahren beruhen. Sie setzen sich aus einem Modell (z. B. einer grafischen Repräsentation des Lösungselements) und einem wiederkehrenden Muster aus in Relation stehenden Eigenschaften (z. B. Eigenschaftsnetzwerke oder mathematische Formeln) zusammen.

In Abbildung 5-9 ist ein Beispiel eines fertigungsintegrierenden Design Patterns dargestellt. Das Design Pattern stellt ein Lösungselement dar, das für biegebeanspruchte Profilbauteile verwendet werden kann. Dessen Besonderheit liegt in den 
integral gefertigten spaltgebogenen Flanschen, die das Flächenträgheitsmoment des Profilquerschnitts und damit dessen Biegesteifigkeit erhöhen. Das Design Pattern ist aus zwei Bestandteilen - Modell und beschreibendes Eigenschaftsmuster - aufgebaut. Das in Form eines einfachen Eigenschaftsnetzwerks dargestellte Eigenschaftsmuster beschreibt den allgemeingültigen Zusammenhang zwischen der Biegesteifigkeit $E I$ und den unabhängigen Eigenschaften einschließlich der verfahrensinduzierten Eigenschaft Flanschlänge $l$ des spaltgebogenen Flansches.

Aufgrund ihrer Allgemeingültigkeit können fertigungsintegrierende Design Patterns in beliebigen Projekten auf unterschiedlichen Ebenen der Produktkonkretisierung verwendet werden. ${ }^{223}$

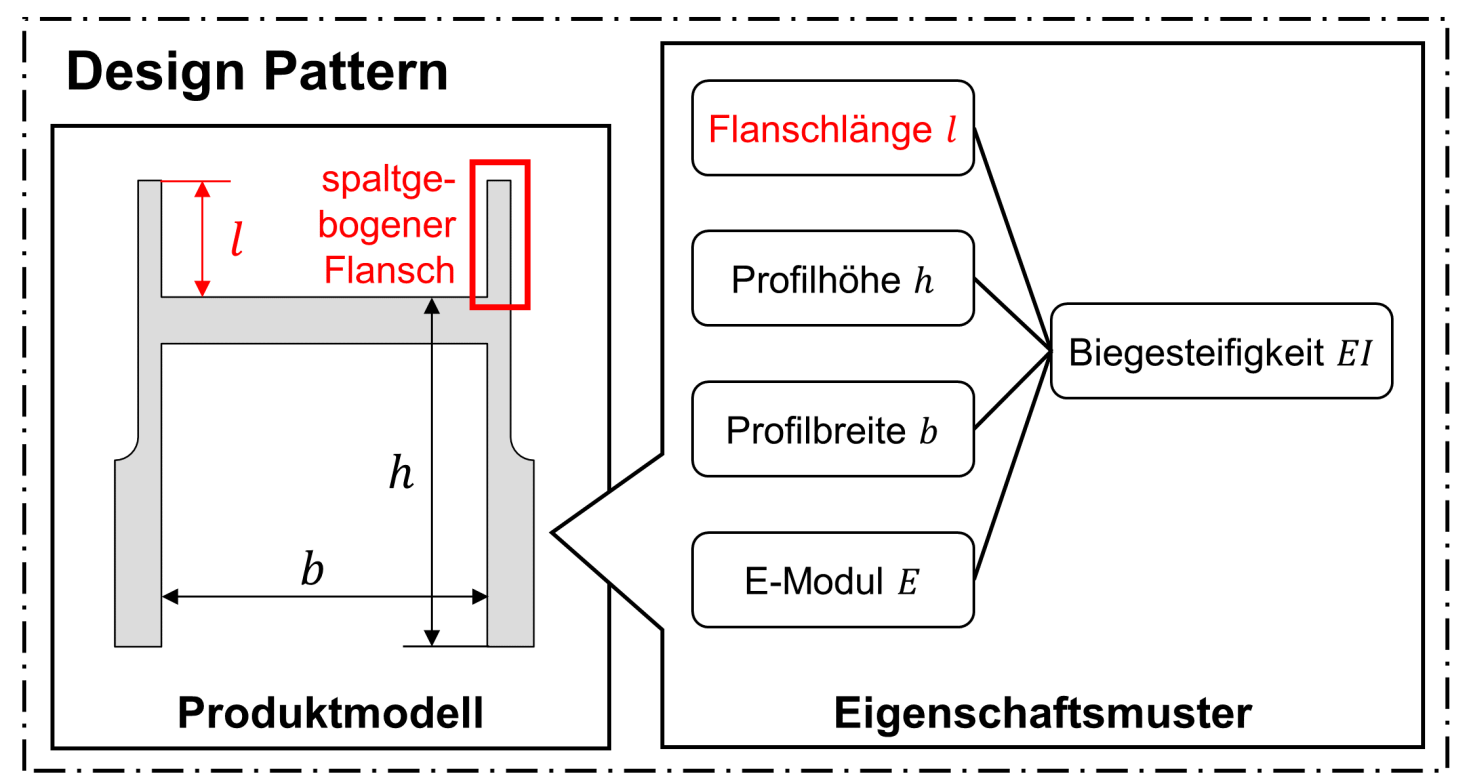

Abbildung 5-9: Kernbestandteile von fertigungsintegrierenden Design Patterns: Modell und wiederkehrendes Eigenschaftsmuster

\subsubsection{Erarbeiten von Design Patterns - Design Pattern Matrix}

Fertigungsintegrierende Design Patterns bieten die Möglichkeit, fertigungstechnologisches Wissen in einer formalisierten Form der Produktentwicklung bereitzustellen. Deren Erarbeitung bedarf einer Verknüpfung von Eigenschaften, die aus den Fertigungsprozessen antizipiert wurden, mit Eigenschaften, die die Produktfunktion beschreiben. Die Verknüpfung muss im Einklang mit der Definition von Design Patterns auf zwei Ebenen stattfinden. Zum einen müssen die verfahrens-

${ }^{223}$ Vgl. Wagner et al. (2016), S. 741 in Anlehnung an Gamma (2007). 
induzierten Eigenschaften mit Eigenschaften, die die Produktfunktion beschreiben, verknüpft werden. Zum anderen müssen entsprechende Modellelemente in einem das Design Pattern charakterisierenden Modell verknüpft werden. Da die Vernetzung von Eigenschaften und Modellelementen im Sinne der Design Patterns jedoch nicht zwangsweise unabhängig voneinander ablaufen kann, bedarf es eines Hilfsmittels, das die gleichzeitige Verknüpfung von Modellelementen und Eigenschaften ermöglicht.

Die sogenannte Design Pattern Matrix ${ }^{224}$ enthält in den Spaltenköpfen Angaben zu den Fertigungsverfahren, die im Rahmen des vorausgegangenen Antizipierens ermittelt wurden. Dies umfasst verfahrensinduzierte Gestalteigenschaften und Gestaltelemente. Sie stellen die Informationen zur Fertigungstechnologie dar, die der Entwickler für die Aufbereitung geeigneter Lösungselemente benötigt. In den Zeilenköpfen werden das Produkt betreffende Angaben eingetragen. Dies sind in erster Linie Funktionsträger und zugehörige Eigenschaften. Den Funktionsträgern sind Modelle und Eigenschaftsrelationen hinterlegt, die deren Funktion genauer beschreiben. Diese können aus verschiedenen Quellen, wie z. B. Lösungssammlungen, Konstruktionskatalogen, aus der Analyse bestehender Produkte oder aus einer vorgelagerten Konzeptphase stammen. Es handelt sich dabei um Elemente, die noch nicht auf Basis einer konkreten Fertigungstechnologie ausgestaltet wurden. ${ }^{225}$ Abbildung 5-10 zeigt den schematischen Aufbau der Design Pattern Matrix am Beispiel der folgenden Funktionsträger: Tragelemente (Aufnahme von Lasten), Führungselemente (Führen des Schlittens) und Verschraubung (Aufhängung des Leichtkransystems). Demgegenüber sind verfahrensinduzierte Gestaltelemente des Spaltbiegens (Spabi), des Spaltprofilierens in Kombination mit Walzprofilieren (Spapro + Wapro) sowie in Kombination mit Fräsen (Spapro + HSC) aufgetragen, die im Rahmen des Antizipierens der Fertigungsprozesse erarbeitet wurden.

\footnotetext{
${ }^{224}$ Vgl. Roos et al. (2017a), S. 13. Im Kontext dieser Arbeit ist darunter stets eine fertigungsintegrierende Design Pattern Matrix zu verstehen.

${ }^{225}$ Vgl. Roos et al. (2017a), S. 13.
} 


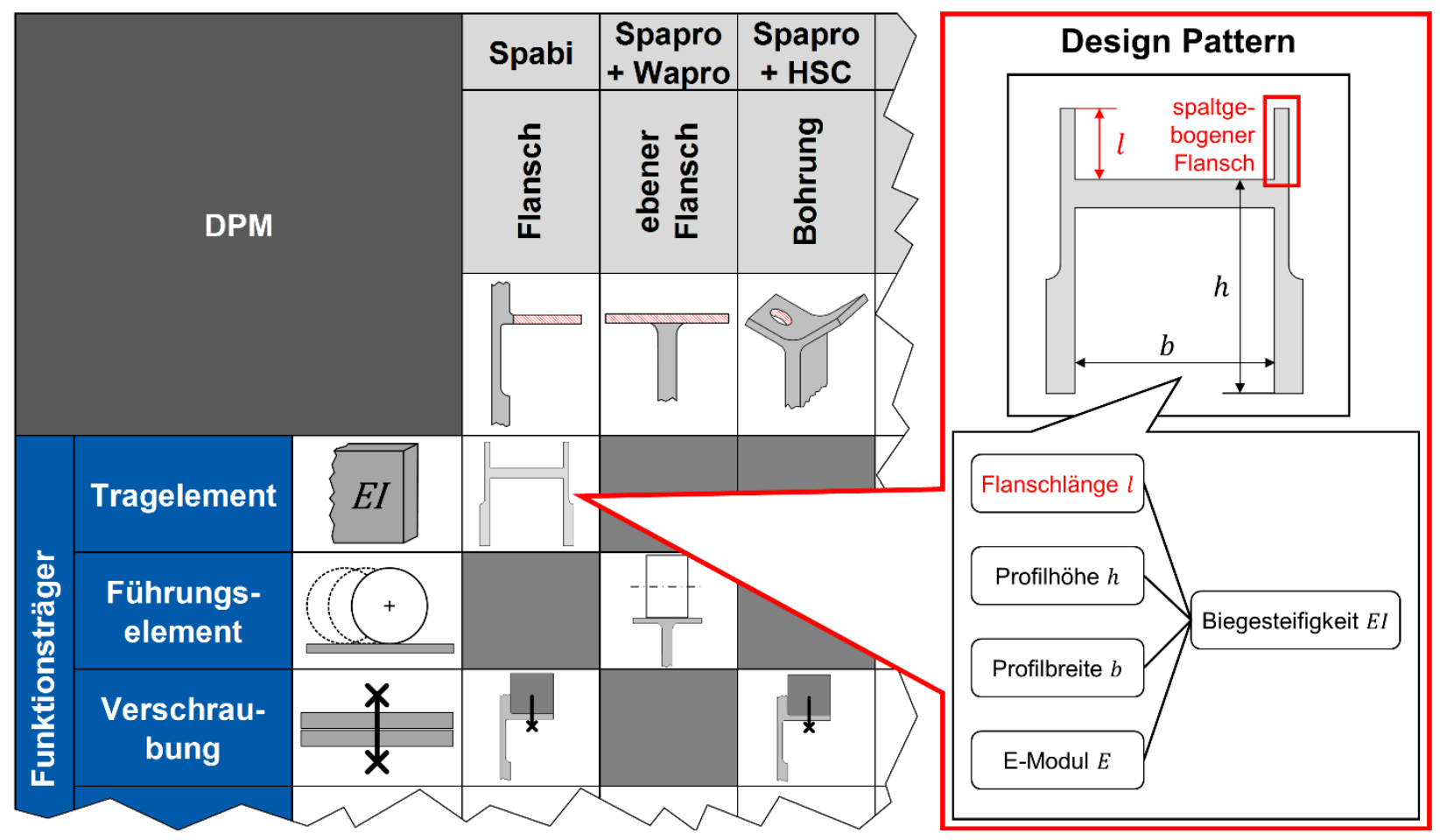

Abbildung 5-10: Aufbau der Design Pattern Matrix

Die gewählten Verfahrenskombinationen in den Spaltenköpfen lassen sich im Rahmen einer kontinuierlichen Fließfertigung umsetzen. ${ }^{226}$ Durch systematische Verknüpfung der Angaben in den Spalten- und Zeilenköpfen in den Zellen der Matrix werden die verfahrensinduzierten Eigenschaften mit den Eigenschaften der Funktionsträger verknüpft (siehe Abbildung 5-10). Im gleichen Zug werden die Funktionsträger mit den verfahrensinduzierten Gestaltelementen verknüpft. Den Funktionsträgern wird dadurch nicht nur eine Gestalt, d. h. eine geometrische und werkstoffliche Ausprägung, zugeordnet. Das Ergebnis sind fertigungsintegrierende Design Patterns, die in den jeweiligen Zellen der Design Pattern Matrix zu finden sind. Abbildung 5-10 greift dazu das bereits vorgestellte Design Pattern auf. Dieses ist vor allem dadurch charakterisiert, dass die spaltgebogenen Flansche in möglichst hohem Abstand zur neutralen Faser des biegebelasteten Profils angeordnet sind, um eine möglichst hohe Bauteilbiegesteifigkeit zu erreichen. Die rot markierte Eigenschaft stellt den Einfluss des Fertigungsverfahrens auf dieses konkrete Lösungselement dar. Zellen, für die keine aussichtsreichen Design Patterns zu finden sind, werden im Sinne einer möglichst effizienten Bearbeitung vorerst 
zurückgestellt (grau markierte Zellen in Abbildung 5-10). Ähnliches findet sich bereits in Form einer alternierenden Kombination und Auswahl im Rahmen der Methode der Systematischen Variation. ${ }^{227}$

Die in der Design Pattern Matrix verorteten Lösungselemente zeichnen sich durch eine teils sehr unterschiedliche Granularität aus. Neben den einzelnen Design Patterns, die zellenweise zugeordnet sind (siehe Einzellösung in Abbildung 5-11) besteht auch die Möglichkeit, Design Patterns zu identifizieren, die sich aus einer Kombination mehrerer Zellen zusammensetzen (siehe Lösungskombination in Abbildung 5-11). Abhängig von der Granularität der gewählten Funktionsträger bzw. verfahrensinduzierter Gestaltelemente können auch mehrere geeignete Lösungselemente in einer Zelle zu finden sein (siehe Mehrfachlösungen in Abbildung $5-11) .{ }^{228}$

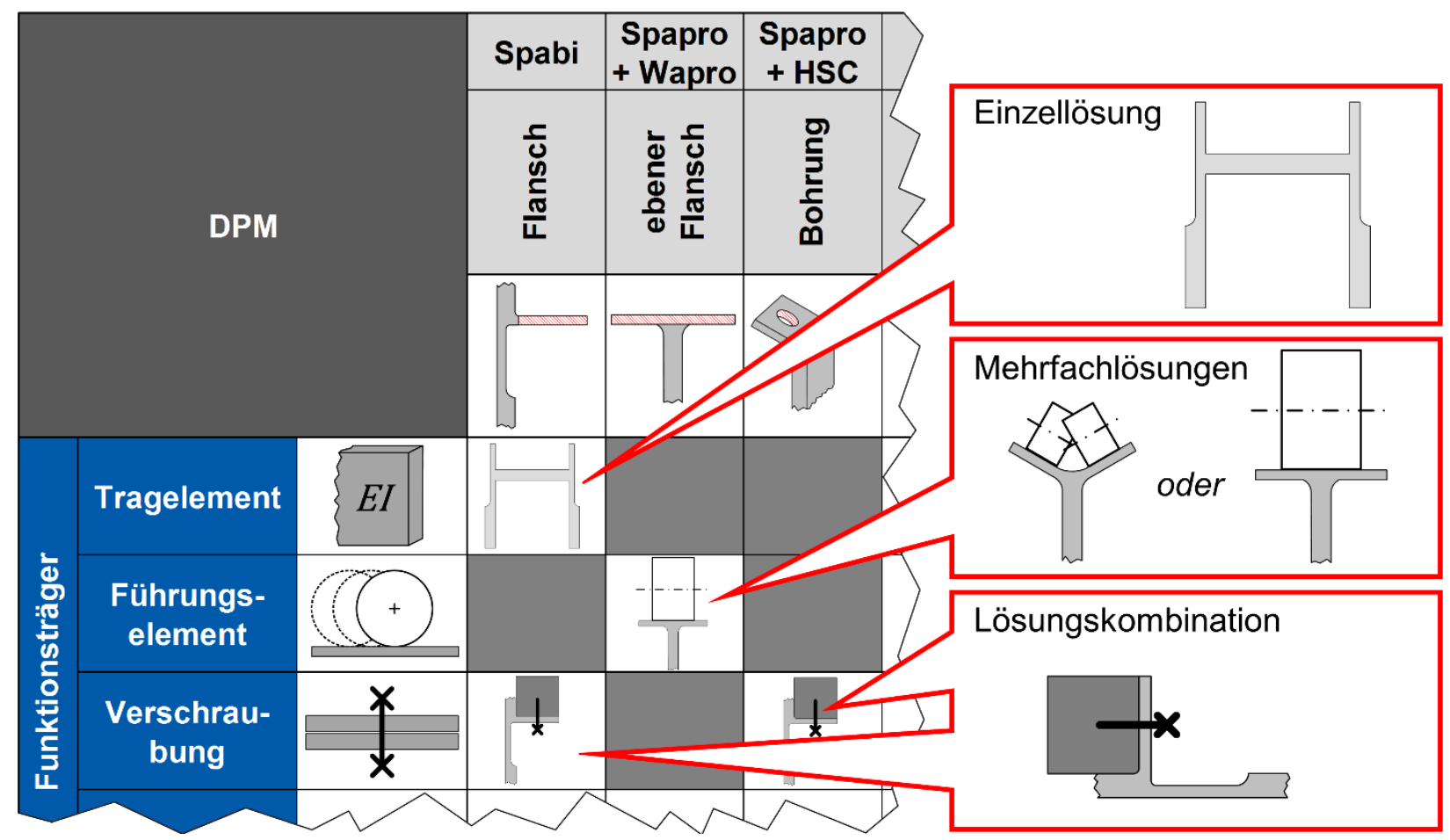

Abbildung 5-11: Vorgehensweisen beim Anwenden der Design Pattern Matrix

Die Verwendung des in Abbildung 5-11 beispielhaft dargestellten Design Patterns für die Entwicklung eines Leichtkransystems führt zu der in Abbildung 5-12 dargestellten Produktlösung, die die Möglichkeiten der Fertigung gezielt ausschöpft. 

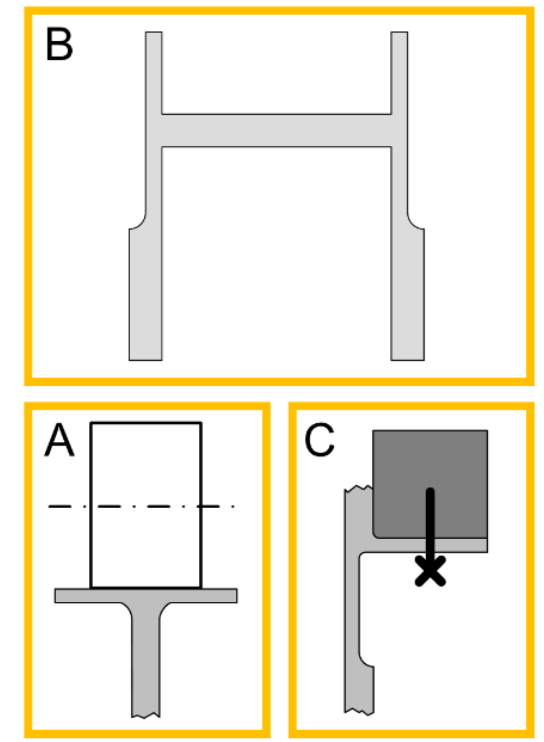
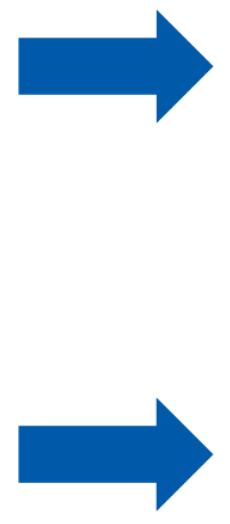

Abbildung 5-12: Vorgehensweisen beim Anwenden der Design Pattern Matrix 229

Das Erarbeiten fertigungsintegrierender Design Patterns auf Basis der DPM entspricht in seinen Grundzügen einem heuristischen Vorgehen. ${ }^{230}$ Während sowohl das vorausgehende Antizipieren und das Zurückgreifen auf Quellen für Funktionsträger als auch das strukturierte Gegenüberstellen der entsprechenden Informationen einer klaren Systematik unterliegt, ist das eigentliche Generieren von Lösungen ein stärker kreativ geprägter Arbeitsschritt. Durch die systematische Vorarbeit werden die Ideen des Entwicklers in geordnete Bahnen gelenkt, sodass die Lösungsgenerierung bestmöglich unterstützt wird.

Die fertigungsintegrierenden Design Patterns stellen im Rahmen des gewählten Fertigungsverfahrens allgemeingültige Lösungselemente dar, die bereits geometrische und werkstoffliche Eigenschaften aufweisen. Sie bieten dennoch das Potenzial, die Produktentwicklung auch auf den abstrakteren Ebenen der Produktkonkretisierung und damit vor allem in den frühen Entwicklungsphasen zu unterstützen (siehe Beispiele in Kapitel 6).

Eine Abwandlung der Design Pattern Matrix findet sich in Form der Manufacturing Integrated Property Matrix (MIPM) ${ }^{231}$, die z. B. für ein systematisches Gegenüberstellen von Eigenschaften verwendet werden kann und $u$. a. eine Operationalisierung des von GRAMLICH vorgestellten Mappings ${ }^{232}$ ermöglicht.

229 In Auszügen aus Gramlich et al. (2015), S. 8.

${ }^{230} \mathrm{Vgl}$. Birkhofer (1980), S. $93 \mathrm{f}$.

${ }^{231}$ Vgl. Roos et al. (2017a), S. $15 \mathrm{ff}$.

232 Vgl. Gramlich (2013), S. 124 ff. 


\subsubsection{Process Integrated Design Guidelines}

Um den Entwickler bei der Lösungsfindung zu unterstützen, müssen Design Patterns für konkrete Problemstellungen zugänglich gemacht werden, sodass der Entwickler auch die Konsequenzen, die sich aus deren Anwendung ergeben, abschätzen kann. Zu diesem Zweck können Richtlinien, bzw. im Englischen „Guidelines“ eingesetzt werden, die auf den Inhalten der Design Patterns beruhen. Im Rahmen einer fertigungsintegrierenden Entwicklungsmethodik werden sogenannte „Process Integrated Design Guidelines“ (PIDG) benötigt, die den Charakteristika fertigungsintegrierender Lösungselemente gerecht werden (siehe Tabelle $5-1) \cdot{ }^{233}$

Die PIDGs geben in Anknüpfung an die Design Patterns an, wie verfahrensinduzierte Gestaltelemente (rot und fett gedruckt in Tabelle 5-1) und deren Eigenschaften (rot in Tabelle 5-1) zur Realisierung bzw. Erweiterung der Produktfunktion (blau und fett gedruckt in Tabelle 5-1) beitragen können. Durch das Zuweisen einer eindeutigen Bezeichnung wird nicht nur die Zugänglichkeit der einzelnen PIDGs sichergestellt, sondern auch das zugrunde liegende Problem adressiert (siehe Tabelle 5-1). Dazu kommen die eigentliche Konstruktionsempfehlung und die Angabe von Konsequenzen, die aus deren Berücksichtigung resultieren. Eine zusätzliche Erklärung weist auf die wesentlichen Einflüsse bzw. Zusammenhänge der zugrunde liegenden Fertigungsverfahren hin, ohne die der Spielraum für konstruktive Festlegungen des Entwicklers nur unzureichend abgeschätzt werden kann. ${ }^{234}$ Tabelle 5-1 gibt eine Übersicht möglicher PIDGs, die bei der Verwendung der Fertigungsverfahren Spaltprofilieren bzw. Spaltbiegen ${ }^{235}$ eingesetzt werden können, um die Produktlösung im Hinblick auf ihre Funktion zu konsolidieren oder auch zu erweitern. PIDGs für weitere Fertigungsverfahren finden sich in Anhang C.

„PIDG Kraftübertragung“ beruht beispielsweise auf dem in Abbildung 5-10 dargestellten fertigungsintegrierenden Design Pattern. Im Fokus dieser Guideline steht die Kraftübertragung in profilartigen Bauteilen. Durch gezieltes Einbringen spaltgebogener Flansche, die eine erhöhte Beanspruchbarkeit aufweisen, in Bereichen, die unter einer hohen Beanspruchung stehen, kann die Beanspruchbarkeit der kompletten Tragstruktur erhöht werden. Die Erklärung dieser PIDG gibt Auskunft darüber, welche grundsätzlichen Einflussmöglichkeiten dem Entwickler zur

\footnotetext{
${ }^{233} \mathrm{Vgl}$. Wagner et al. (2016).

${ }^{234}$ Vgl. Wagner et al. (2016), S. $741 \mathrm{ff}$.

${ }^{235}$ Vgl. Ringler, Groche (2008), S. 64 f.
} 
Verfügung stehen, um die exakten Ausprägungen der verfahrensinduzierten Eigenschaften spaltprofilierter Flansche zu beeinflussen.

Tabelle 5-1: Beispiele für Process Integrated Design Guidelines ${ }^{236}$

\begin{tabular}{|c|c|c|c|}
\hline $\begin{array}{l}\text { Bezeich- } \\
\text { nung }\end{array}$ & $\begin{array}{l}\text { Konstruktions- } \\
\text { empfehlung }\end{array}$ & Konsequenz & Erklärung \\
\hline $\begin{array}{l}\text { PIDG } \\
\text { Linearfüh- } \\
\text { rung }\end{array}$ & $\begin{array}{l}\text { Nutze spaltprofilierte } \\
\text { und spaltgebogene } \\
\text { Flansche mit ihrer erhöh- } \\
\text { ten Wälzfestigkeit, um } \\
\text { Wälzkontaktflächen in } \\
\text { Linearführungen zu reali- } \\
\text { sieren. }\end{array}$ & $\begin{array}{l}\text { erhöhte Lebens- } \\
\text { dauer der Wälz- } \\
\text { kontakte sowie } \\
\text { erhöhte Oberflä- } \\
\text { chenqualität }\end{array}$ & $\begin{array}{l}\text { Das UFG Gefüge } \\
\text { zeichnet sich durch } \\
\text { eine hohe Härte und } \\
\text { geringe Oberflächen- } \\
\text { rauheit aus in Abhän- } \\
\text { gigkeit des Halbzeug- } \\
\text { werkstoffs und be- } \\
\text { stimmter Prozesspa- } \\
\text { rameter. }\end{array}$ \\
\hline $\begin{array}{l}\text { PIDG } \\
\text { Kraftüber- } \\
\text { tragung }\end{array}$ & $\begin{array}{l}\text { Nutze spaltprofilierte } \\
\text { und spaltgebogene } \\
\text { Flansche mit ihrer erhöh- } \\
\text { ten Festigkeit in hoch be- } \\
\text { anspruchten Bereichen } \\
\text { innerhalb profilartiger } \\
\text { Bauteile, die der Kraft- } \\
\text { übertragung dienen. }\end{array}$ & $\begin{array}{l}\text { erhöhte Bean- } \\
\text { spruchbarkeit }\end{array}$ & $\begin{array}{l}\text { Die UFG Gefü- } \\
\text { gestruktur weist eine } \\
\text { erhöhte Festigkeit auf } \\
\text { in Abhängigkeit des } \\
\text { Halbzeugwerkstoffs } \\
\text { und bestimmter Pro- } \\
\text { zessparameter. }^{238}\end{array}$ \\
\hline $\begin{array}{l}\text { PIDG } \\
\text { Kerbwir- } \\
\text { kung }\end{array}$ & $\begin{array}{l}\text { Nutze spaltprofilierte } \\
\text { und spaltgebogene } \\
\text { Flansche mit ihrer gerin- } \\
\text { geren Kerbempfindlich- } \\
\text { keit, um Funktionsele- } \\
\text { mente in profilartige Bau- } \\
\text { teile zu integrieren. }\end{array}$ & $\begin{array}{l}\text { erhöhte Belast- } \\
\text { barkeit des } \\
\text { Formelements }\end{array}$ & $\begin{array}{l}\text { Der Vergleich ver- } \\
\text { schiedener Wöhler- } \\
\text { Kurven deutet auf } \\
\text { eine geringere Kerb- } \\
\text { empfindlichkeit des } \\
\text { UFG Gefüges hin. }{ }^{239}\end{array}$ \\
\hline
\end{tabular}

Gerade am Beispiel der fertigungsintegrierenden Design Patterns und der PIDGs wird deutlich, dass die Kenntnis der Wirkzusammenhänge im Prozesskontext unabdingbar für das Verständnis konstruktiver Festlegungen im Rahmen der Produktentwicklung ist. Vor allem für Wirkzusammenhänge, die durch den Operanden im Anfangszustand dominiert werden, hat der Entwickler umfangreiche Möglichkeiten der Einflussnahme. Seine konstruktiven Festlegungen betreffen damit nicht

${ }^{236}$ In Anlehnung an Gramlich et al. (2015).

${ }^{237} \mathrm{Vgl}$. Ahmels et al. (2017).

${ }^{238} \mathrm{Vgl}$. Ahmels et al. (2017).

${ }^{239} \mathrm{Vgl}$. Ahmels et al. (2017). 
mehr nur das Produkt, sondern auch die zu verwendenden Halbzeuge. So weisen vor allem die Erklärungen zu „PIDG Linearführung“ und „PIDG Kraftübertragung“ in Tabelle 5-1 darauf hin, dass verschiedene Prozessparameter sowie das Werkzeugsystem einen hohen Einfluss auf das UFG Gefüge und damit letztlich auf entsprechende konstruktive Festlegungen haben. Dennoch beeinflusst auch der Halbzeugwerkstoff die jeweiligen Ausprägungen der verfahrensinduzierten Eigenschaften. Diesen kann der Entwickler im Zuge der Produktkonkretisierung gezielt festlegen. Die PIDG zeigt inm somit auf, dass er durch eine geeignete Wahl des Werkstoffs einen wesentlichen Einfluss auf die Eigenschaften der Lösung zum jeweiligen Problem hat. Das Erarbeiten von PIDGs erfordert trotz der zugrunde liegenden Methodik meist interdisziplinäre Projektteams u. a. mit Experten aus den Bereichen Fertigung, Materialwissenschaft, Betriebsfestigkeit und Produktentwicklung, um eine möglichst umfassende Wissensbasis zu schaffen.

\subsection{Fazit - Modellbasierte Entwicklungsmethodik}

Die Angabe einzelner Arbeitsschritte im Rahmen eines übergeordneten Vorgehens und darauf abgestimmter Methoden und Tools präzisiert die ursprüngliche Idee, fertigungstechnologisches Wissen direkt in der Produktentwicklung zu verarbeiten, zu einer umfassenden Methodik für die Entwicklung fertigungsintegrierender Produktlösungen. Die Bedeutung des Ansatzes für die Produktentwicklung beruht vor allem auf einer konsistent aufgebauten Modellwelt (siehe Kapitel 4), mittels derer Produkte und Prozesse auf der Basis von Eigenschaften in aufeinander abgestimmten Modellen beschrieben werden können. Erst damit wird es möglich, Prozesse des Produktlebenslaufs in geeigneter Weise zu antizipieren und entsprechende Schlüsse für die Produktentwicklung zu ziehen. Die Anwendung des Antizipierens im Rahmen der fertigungsintegrierenden Entwicklungsmethodik erlaubt es zudem, den zur Verfügung stehenden Lösungsraum gezielt um fertigungsintegrierende Lösungselemente zu erweitern. Darauf aufbauend können verschiedene innovative Lösungsalternativen entwickelt werden. Durch das Generieren fertigungsintegrierender Lösungselemente ergeben sich neue Möglichkeiten für die Umsetzung der Produktfunktion.

Insbesondere das Antizipieren und die zugrunde liegende einheitliche Modellwelt stellen wichtige Bausteine der fertigungsintegrierenden Entwicklungsmethodik dar. Das aufbereitete fertigungstechnologische Wissen ist damit nicht nur die Grundlage für die Lösungsfindung und -konkretisierung im Rahmen der Produkt- 
entwicklung. Durch die Verwendung geeigneter Modelle und angepasster Arbeitsschritte kann dieses Wissen auch für die Prozessentwicklung verwendet werden. Erste Ansätze zur Konkretisierung von Einsatzprozessen wurden in Abschnitt 4.3 gezeigt. Gegenüber der Konkretisierung von Einsatzprozessen muss die Fertigungsprozessentwicklung zwangsweise parallel bzw. gleichzeitig zur Produktentwicklung ablaufen, da jedes Beeinflussen im Kontext des Antizipierens auf Festlegungen beruht, die in Bezug auf Fertigungsprozesse gemacht werden. Hierfür wird jedoch eine entsprechende Operationalisierung benötigt, die die Prozessentwicklung samt der dazu benötigten Modelle beschreibt. 


\section{Evaluierung der fertigungsintegrierenden Entwicklungsmethodik}

Aufbauend auf den in Kapitel 4 vorgestellten Modellen wurde in Kapitel 5 eine fertigungsintegrierende Entwicklungsmethodik vorgestellt, die es ermöglicht, Fertigungspotenziale für die Produktentwicklung in Form fertigungsintegrierender Design Patterns auszuschöpfen. Die Anwendung dieser Methodik und die daraus resultierenden Vorteile für die Lösungsfindung während der Produktentwicklung werden im Folgenden anhand zweier Evaluierungsbeispiele verdeutlicht. Das erste Beispiel umfasst das Erschließen von Fertigungspotenzialen für innenhochdruckumgeformte Fahrradrahmen (siehe Abschnitt 6.1). Im zweiten Beispiel werden die Möglichkeiten des Spaltprofilierens für die Realisierung von Deckenschienen mit werkzeuglos montierbaren Halteelementen untersucht (siehe Abschnitt 6.2).

Bei den genannten Beispielen wird davon ausgegangen, dass zu Beginn des Entwicklungsprozesses bereits geeignete Fertigungsverfahren zur Realisierung der angedachten Produkte identifiziert wurden (siehe Abbildung 5-6). Nur durch frühzeitig festgelegte Fertigungsverfahren ist es möglich, Fertigungspotenziale systematisch für die Produktentwicklung zu erschließen. Geeignete Ansätze, wie sie bei GRAMLICH oder im Rahmen des Algorithm-Based Product and Process Development zu finden sind, beruhen auf einem frühzeitigen Abgleich von Eigenschaften aus dem Fertigungskontext mit solchen aus dem Funktionskontext des Produkts. Das Mapping dieser Eigenschaften liefert Aussagen darüber, ob die angedachten Funktionsträger des Produkts grundsätzlich realisiert werden können. ${ }^{240}$

\subsection{Beispiel innenhochdruckumgeformter Fahrradrahmen}

Der Fahrradrahmen stellt eine der zentralen Komponenten eines Fahrrads dar. An inm werden nahezu alle Komponenten angebracht, die die Funktion des Gesamtsystems Fahrrad sicherstellen. Dazu zählen u. a. Räder, Lenker, Sattel oder Antrieb. Dadurch übernimmt der Fahrradrahmen direkt oder indirekt eine Vielzahl an zusätzlichen Funktionen, die über seine Hauptfunktion ${ }^{241}$, Kräfte zwischen den Komponenten zu leiten, hinausgehen. Fahrradrahmen werden als Tragstrukturen ausgeführt, die sich in Abhängigkeit des gewählten Werkstoffs meist aus Rohren mit unterschiedlichen Durchmessern, Längen und Wanddicken zusammensetzen.

\footnotetext{
${ }^{240}$ Vgl. Gramlich (2013), S. 123 ff.; Tekkaya et al. (2015), S. 648; Gramlich et al. (2015), S. 4; Lüthen et al. (2017), S. $159 \mathrm{ff}$.

${ }^{241}$ Vgl. Pahl et al. (2007), S. 45
} 
Aus diesem Grund bieten sich Fertigungsverfahren an, die dünnwandige Profilgeometrien herstellen können. Unter anderem ist hier auch das Innenhochdruckumformen (IHU) zu nennen, das neben dem Gewährleisten einer dünnwandigen Profilgeometrie zusätzliche Freiheitsgrade in Bezug auf diskontinuierliche Formelemente ermöglicht (siehe Abbildung 6-1). In Anknüpfung an die in Kapitel 5 beschriebene Methodik sollen im Folgenden fertigungsintegrierende Produktlösungen entwickelt werden, die die Möglichkeiten des Innenhochdruckumformens für die Realisierung von Fahrradrahmen umfassend ausschöpfen.
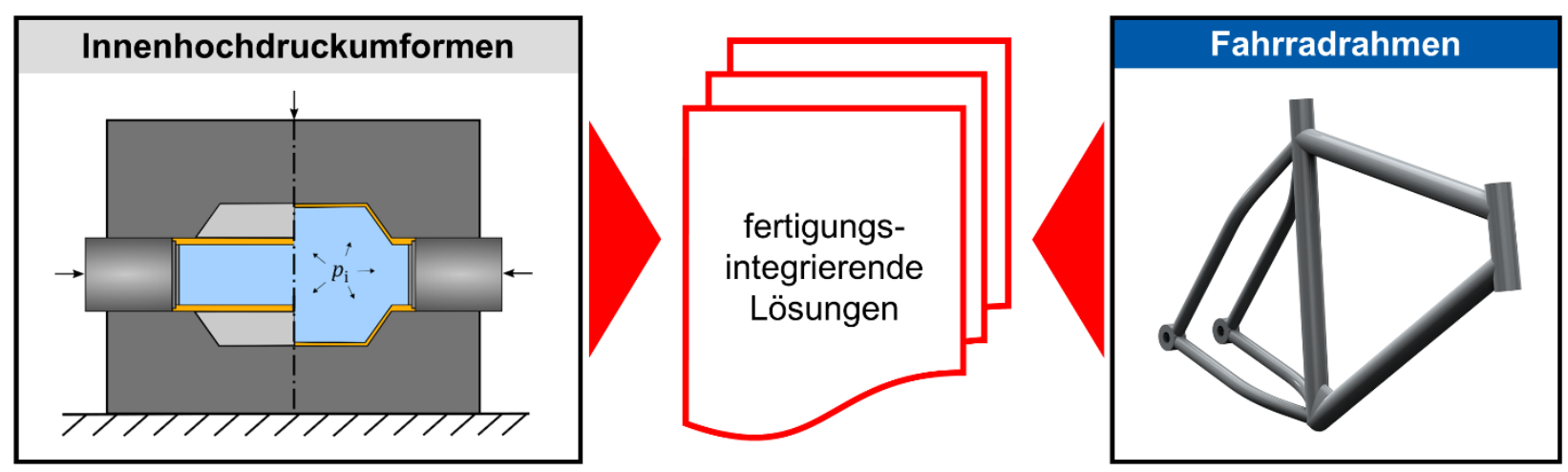

Abbildung 6-1: Erarbeitung fertigungsintegrierender Lösungen für IHU-Fahrradrahmen

Das Ziel der Entwicklung fertigungsintegrierender Produktlösungen für Fahrradrahmen liegt im Folgenden nicht in der Substitution gewöhnlicher Rahmengeometrien, sondern darin, durch gezieltes Ausschöpfen fertigungstechnologischer Potenziale, einen erkennbaren Mehrwert in Bezug auf die Gesamtfunktion des Fahrradrahmens zu schaffen. Dazu soll gezeigt werden, welcher Mehrwert sich aus der Fertigungstechnologie Innenhochdruckumformen für die Realisierung zusätzlicher Funktionen erschließen lässt, die über die eigentliche Kraftleitung des Fahrradrahmens hinausgehen (siehe Abbildung 6-1). Aus den dem Fahrradrahmen benachbarten Komponenten können hierzu geeignete Funktionsträger abgeleitet werden:

- Spritzschutz

- Flaschenhalter

- Gepäckträger

- Handyhalterung

- Kabelführung

- Akkuhalterung

- Motoraufnahme

- Druckspeicher 
Damit ist neben dem Ziel des Vorgehens auch der Betrachtungsrahmen in Form der bereits bekannten Funktionsträger des zu entwickelnden Produkts und des festgelegten Fertigungsverfahrens Innenhochdruckumformen klar umrissen (Arbeitsschritte 1 und 2 der fertigungsintegrierenden Entwicklungsmethodik).

\subsubsection{Antizipieren des Innenhochdruckumformens}

Nachdem das Ziel bekannt ist und der Betrachtungsrahmen festgelegt ist, müssen die Fertigungsprozesse antizipiert werden. Dafür eignet sich vor allem das VPModell, um aus fertigungstechnologischem Wissen die benötigten Informationen in Form von Eigenschaften zu ermitteln (Arbeitsschritt 3 der Methodik). Zum Zweck der Erarbeitung fertigungsintegrierender Produktlösungen sind das vor allem die verfahrensinduzierten Gestaltelemente und deren Eigenschaften.

Die Modellierung und Analyse des Verfahrensprinzips des Innenhochdruckumformens zeigt, dass mittels dieses Verfahrens unterschiedliche Gestaltelemente realisierbar sind (Arbeitsschritt 4 der Methodik). Dies sind u. a. gezielt eingebrachte Rohrkrümmungen, Dome oder auch Rohraufweitungen, wie sie in Abbildung 6-2 dargestellt sind.

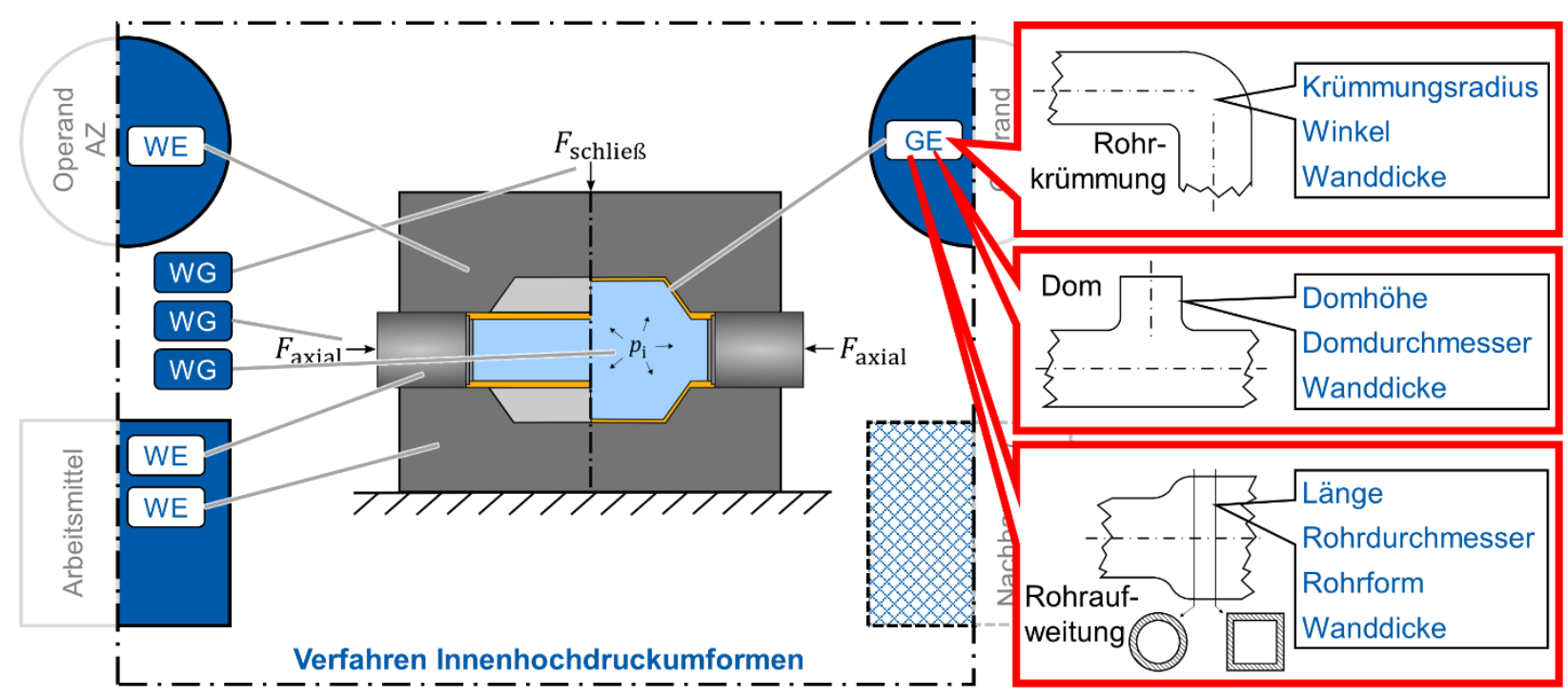

Abbildung 6-2: Antizipieren des Innenhochdruckumformens

Die verfahrensinduzierten Eigenschaften beschreiben im Kontext dieses Fertigungsverfahrens vor allem die Änderungen in Bezug auf die Bauteilgeometrie, wie z. B. die Höhe, den Durchmesser oder die Wanddicke des neu ausgebildeten Doms. Weitere Gestaltelemente samt ihren Eigenschaften finden sich in der im 
nächsten Abschnitt dargestellten DPM. An dieser Stelle sind vor allem die Merkmale der Eigenschaften von Interesse. Zwar limitieren die Grenzen möglicher Merkmalsausprägungen der verfahrensinduzierten Eigenschaften auch die Eigenschaften des späteren Produkts. Da hier jedoch in erster Linie neuartige prinzipielle Lösungen in den frühen Entwicklungsphasen, d. h. im Zuge der Konzepterarbeitung, generiert werden sollen, genügt vorerst die Kenntnis der Merkmale.

\subsubsection{Aufbereiten der Ergebnisse aus dem Antizipieren}

Nach dem Antizipieren folgt der eigentliche Integrationsschritt. Dazu müssen die identifizierten Gestaltelemente und deren Eigenschaften in Form fertigungsintegrierender Lösungselemente für die Produktentwicklung aufbereitet und beurteilt werden (Arbeitsschritte 5 und 6 der Methodik).

\section{Design Pattern Matrix}

Fertigungsverfahrensinduzierte Gestaltelemente und deren Eigenschaften werden mittels der DPM den zu realisierenden Funktionsträgern und deren Eigenschaften gegenübergestellt. Die Erarbeitung fertigungsintegrierender Design Patterns findet in den Zellen der DPM auf Basis einer Verknüpfung dieser Gestaltelemente und Funktionsträger und ihrer jeweiligen Eigenschaften statt. Auf diese Weise werden nicht nur das fertigungstechnologische Produkt-Wissen mit vorhandenem Produkt-Wissen verknüpft, sondern darüber hinaus erste Lösungsalternativen ermittelt (Arbeitsschritt 5 der Methodik). Abbildung 6-3 zeigt einen Ausschnitt der fertigungsintegrierenden DPM, in der die genannten Funktionsträger mit Gestaltelementen des Innenhochdruckumformens verknüpft werden. Ergänzend wird auch das Schweißen als ein Verfahren aufgeführt, das sich sehr gut mit dem Innenhochdruckumformen kombinieren lässt, um geschlossene Kammern zu erzeugen. Durch Kombination der Funktionsträger mit den Gestaltelementen können unterschiedliche Lösungselemente generiert werden, die sich teilweise über mehrere Zellen einer Zeile der Matrix erstrecken. Die einzelnen Lösungselemente sind mit der Zahl 1 beginnend durchnummeriert. Die ausgegrauten Zellen wurden vorerst zurückgestellt. Für den Funktionsträger Flaschenhalter finden sich drei unterschiedliche Lösungselemente, die in Abbildung 6-3 näher dargestellt sind. Die von Hand erarbeiteten Skizzen zeigen drei Möglichkeiten, einen Flaschenhalter im Unterrohr des Fahrradrahmens zu integrieren. Sie stellen dabei lediglich Lösungsalternativen dar, die zu diesem Zeitpunkt noch keiner Beurteilung hinsichtlich ihrer 
Eignung für das eigentliche Entwicklungsprojekt unterzogen wurden. Die vollständige DPM findet sich in Anhang A.

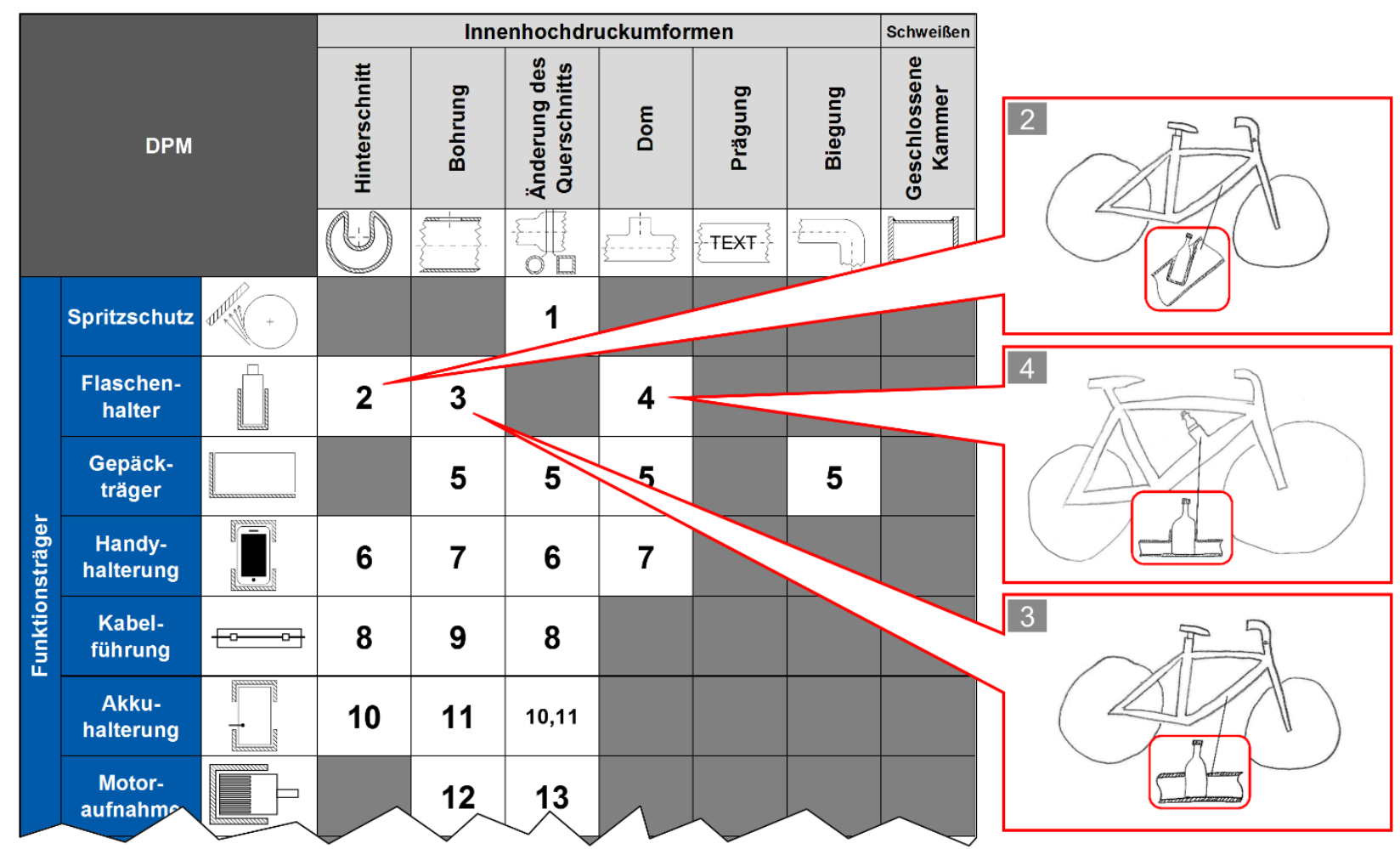

Abbildung 6-3: Design Pattern Matrix für innenhochdruckumgeformte Fahrradrahmen

Zur Vereinfachung der eigentlichen Erarbeitung fertigungsintegrierender Design Patterns wurden die zugehörigen Eigenschaften in Abbildung 6-3 nicht dargestellt. Diese finden sich auszugweise in Form eines Eigenschaftsnetzwerks für das Beispiel eines innenhochdruckumgeformten Flaschenhalters in Abbildung 6-4 wieder.

\section{Varianten innenhochdruckumgeformter Flaschenhalter}

Die DPM zeigt mehrere Möglichkeiten auf, die Funktionsträger in Form von fertigungsintegrierenden Lösungselementen zu realisieren. Zur Erläuterung der weiteren Ausarbeitung der Lösungselemente wird im Folgenden das Beispiel eines innenhochdruckumgeformten Flaschenhalters beschrieben. Abbildung 6-3 zeigt bereits drei Lösungselemente, die unterschiedliche Möglichkeiten zur Integration eines Flaschenhalters in das Unterrohr eines Fahrradrahmens aufzeigen. Die skizzenhaft erfassten Lösungselemente bedürfen einer weiteren Konkretisierung, um im Anschluss zu beurteilen, ob diese einen Mehrwert für das eigentliche Entwicklungsprojekt bieten. Dazu werden durch den Produktentwickler schrittweise weitere Eigenschaften festgelegt, deren Merkmale anhand eines Referenzrohres (siehe Anhang A) ermittelt wurden. Abbildung 6-4 zeigt fünf Entwürfe in Form von 
Design Patterns, die als Varianten aus den drei skizzierten Lösungselementen hervorgehen. Das zugehörige Eigenschaftsnetzwerk erläutert für jedes der fünf Design Patterns, wie die Funktion des Flaschenhalters, charakterisiert durch ein Aufnahmevolumen $V$, von den Eigenschaften Durchmesser $D$, Länge $l$ und Anzahl der Formelemente ${ }^{242} n$ abhängt. Im Sinne fertigungsintegrierender Design Patterns werden sowohl der Durchmesser $D$ als auch die Länge $l$ durch die verfahrensinduzierten Eigenschaften der jeweiligen Gestaltelemente der einzelnen Varianten festgelegt (siehe rote Farbgebung in Abbildung 6-4). Die verschiedenen Design Patterns unterscheiden sich $u$. a. in den benötigten Bauräumen und dem Fertigungsaufwand. Anhand dieser Eigenschaften gilt es, im Folgenden eine Beurteilung der Lösungsalternativen vorzunehmen.

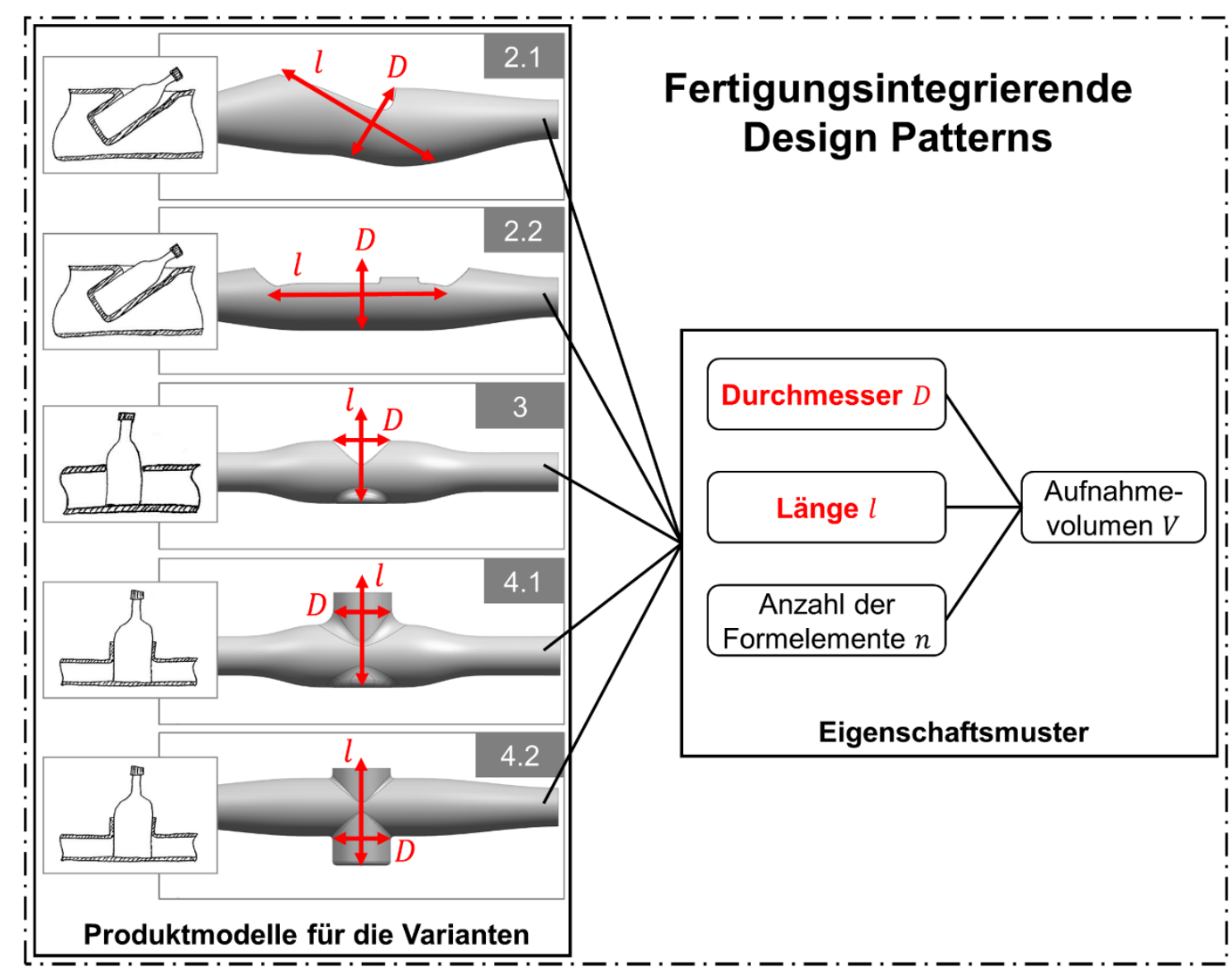

Abbildung 6-4: Design Patterns für innenhochdruckumgeformte Flaschenhalter

Zusätzlich zu den erarbeiteten Design Patterns können PIDGs angegeben werden, die auf den wesentlichen Zusammenhängen der Design Patterns beruhen (siehe Tabelle 6-1).

242 Unter Formelementen wird in diesem Beispiel ein einzelner Flaschenhalter verstanden. 
Tabelle 6-1: Process Integrated Design Guidelines für das Innenhochdruckumformen

\begin{tabular}{|c|c|c|c|}
\hline $\begin{array}{l}\text { Bezeich- } \\
\text { nung }\end{array}$ & $\begin{array}{l}\text { Konstruktions- } \\
\text { empfehlung }\end{array}$ & Konsequenz & Erklärung \\
\hline $\begin{array}{l}\text { PIDG } \\
\text { Haltevor- } \\
\text { richtung } 1\end{array}$ & $\begin{array}{l}\text { Nutze innenhochdruck- } \\
\text { umgeformte Dome, um } \\
\text { rotationssymmetrische } \\
\text { Bauteile im Rahmenrohr } \\
\text { von Fahrrädern zu fixie- } \\
\text { ren. }\end{array}$ & $\begin{array}{l}\text { Einzelteile, Ferti- } \\
\text { gungsprozesse } \\
\text { und Montagepro- } \\
\text { zesse können } \\
\text { eingespart wer- } \\
\text { den. }\end{array}$ & $\begin{array}{l}\text { IHU bietet die Mög- } \\
\text { lichkeit Dome auszu- } \\
\text { formen, wobei deren } \\
\text { Höhe insbesondere } \\
\text { durch das Werkzeug } \\
\text { und das Werkstück } \\
\text { beeinflusst wird (siehe } \\
\text { Abbildung 4-13). }{ }^{243} \\
\end{array}$ \\
\hline $\begin{array}{l}\text { PIDG } \\
\text { Haltevor- } \\
\text { richtung } 2\end{array}$ & $\begin{array}{l}\text { Nutze während des IHU- } \\
\text { Prozesses eingebrachte } \\
\text { Bohrungen, um rotati- } \\
\text { onssymmetrische Bau- } \\
\text { teile im Rahmenrohr von } \\
\text { Fahrrädern zu fixieren. }\end{array}$ & $\begin{array}{l}\text { Einzelteile, Ferti- } \\
\text { gungsprozesse } \\
\text { und Montagepro- } \\
\text { zesse können } \\
\text { eingespart wer- } \\
\text { den. }\end{array}$ & $\begin{array}{l}\text { Durch einen Stempel } \\
\text { im IHU-Werkzeug } \\
\text { können in das unter } \\
\text { einem Innendruck ste- } \\
\text { hende Rohr prozess- } \\
\text { integriert Löcher ge- } \\
\text { stanzt werden. }\end{array}$ \\
\hline $\begin{array}{l}\text { PIDG } \\
\text { Haltevor- } \\
\text { richtung } 3\end{array}$ & $\begin{array}{l}\text { Nutze die über der Rohr- } \\
\text { länge variablen Quer- } \\
\text { schnittgeometrien in- } \\
\text { nenhochdruckumge- } \\
\text { formter Rohre, um Hin- } \\
\text { terschnitte zu realisieren, } \\
\text { in denen zusätzliche } \\
\text { Bauteile fixiert werden } \\
\text { können. }\end{array}$ & $\begin{array}{l}\text { Einzelteile, Ferti- } \\
\text { gungsprozesse } \\
\text { und Montagepro- } \\
\text { zesse können } \\
\text { eingespart wer- } \\
\text { den. }\end{array}$ & $\begin{array}{l}\text { Die Größe des Hinter- } \\
\text { schnitts und dessen } \\
\text { Radien werden durch } \\
\text { den Werkstoff des } \\
\text { Halbzeugs, dessen } \\
\text { Wanddicke sowie die } \\
\text { realisierbaren Um- } \\
\text { formkräfte limitiert. }\end{array}$ \\
\hline
\end{tabular}

\section{Beurteilen der Varianten}

Die erarbeiteten Varianten müssen mit Blick auf das Entwicklungsprojekt beurteilt werden (Arbeitsschritte 6 der Methodik). Dieser Schritt wird der Bearbeitung der DPM nachgelagert durchgeführt, da eine Implementierung in die DPM die Komplexität für den Entwickler zu stark erhöhen würde. Zudem wird auf diese Weise zwischen allgemeingültigen Lösungselementen (Design Patterns) und deren projektspezifischer Anwendung unterschieden. Das Beurteilen der fertigungsintegrierenden Design Patterns findet im Folgenden auf Basis einer Punktbewertung statt.

\footnotetext{
${ }^{243} \mathrm{Vgl}$. Bahn et al. (2007), S. 43.

${ }^{244}$ Vgl. Bahn et al. (2007), S. $126 \mathrm{ff}$.

${ }^{245}$ Vgl. Bahn et al. (2007), S. $41 \mathrm{f}$.
} 
Dazu werden aus projektspezifischen Anforderungen Bewertungskriterien abgeleitet (siehe Tabelle 6-2). Jedoch können nicht alle Anforderungen bereits während der hier betrachteten Konzeptfindung in Form von Bewertungskriterien berücksichtigt werden. Das Gewährleisten eines möglichst spielfreien Haltens der Flasche bedingt beispielweise eine detaillierte Toleranzanalyse, die in geeigneter Form erst in späteren Entwicklungsphasen durchgeführt werden kann.

Tabelle 6-2: Bewertungskriterien für innenhochdruckumgeformte Flaschenhalter

\begin{tabular}{ll}
\hline Kriterium & Beschreibung \\
\hline Befestigung & $\begin{array}{l}\text { Für die Einspannung der Trinkflasche ist eine ausreichend } \\
\text { große Einspannlänge notwendig, um auftretende Biegemo- } \\
\text { mente aufnehmen zu können. }\end{array}$ \\
\hline Erreichbarkeit & $\begin{array}{l}\text { Um ein möglichst einfaches Entnehmen der Trinkflasche zu } \\
\text { gewährleisten, sollte der Entnahmeweg möglichst kurz sein. }\end{array}$ \\
\hline Steifigkeit & $\begin{array}{l}\text { Bedingt durch unterschiedliche Belastungsrichtungen sind die } \\
\text { Dehnsteifigkeit, Biegesteifigkeit und Torsionssteifigkeit zu } \\
\text { überprüfen. Die Werte der Variante mit der jeweils höchsten }\end{array}$ \\
& Steifigkeit werden auf eins normiert. \\
\hline Werkstoffaus- & $\begin{array}{l}\text { Die Werkstoffausnutzung wird jeweils für auftretende } \\
\text { nutzung }\end{array}$ \\
& $\begin{array}{l}\text { Zug-/Druck-, Biege- und Torsionsbelastung ermittelt. Dabei } \\
\text { wird der Anteil all derjenigen FE-Knoten ermittelt, deren } \\
\text { Spannung oberhalb von 50 \% der Maximalspannung liegt. }\end{array}$ \\
\hline Masse & $\begin{array}{l}\text { Die Masse der Variante sollte gegenüber der des Referenz- } \\
\text { rohres nicht wesentlich zunehmen. }\end{array}$ \\
\hline Reinigbarkeit & $\begin{array}{l}\text { Um eine leichte Reinigung des Fahrradrahmens zu gewähr- } \\
\text { leisten, sollte das Rohr geschlossen und ohne Hinterschnitte } \\
\text { ausgeführt sein. }\end{array}$ \\
\hline
\end{tabular}

Während für die Beurteilung von Befestigung, Erreichbarkeit, Masse und Reinigbarkeit die Bauteilgeometrie der jeweiligen Variante herangezogen werden kann, sind für die Beurteilung der Steifigkeit sowie der Werkstoffausnutzung präzisere Untersuchungen notwendig. Zu diesem Zweck wurden FE-Simulationen durchgeführt, um unter Last auftretende Spannungen und Verschiebungen zu ermitteln. Eine Übersicht über die zugrunde liegenden Annahmen, Parameter und die Simulationsergebnisse findet sich in Anhang A. Auf Basis der simulierten Verschiebungen unter bekannten Lasten können Aussagen über die Steifigkeit der Varianten in Bezug auf die verschiedenen Lastfälle getroffen werden. Deren Resultate sind in Abbildung 6-5 aufgetragen. Hier zeigt sich, dass vor allem die Variante 2.1 erkennbare Vorteile in Bezug auf Dehn-, Biege- und Torsionssteifigkeit bietet. 


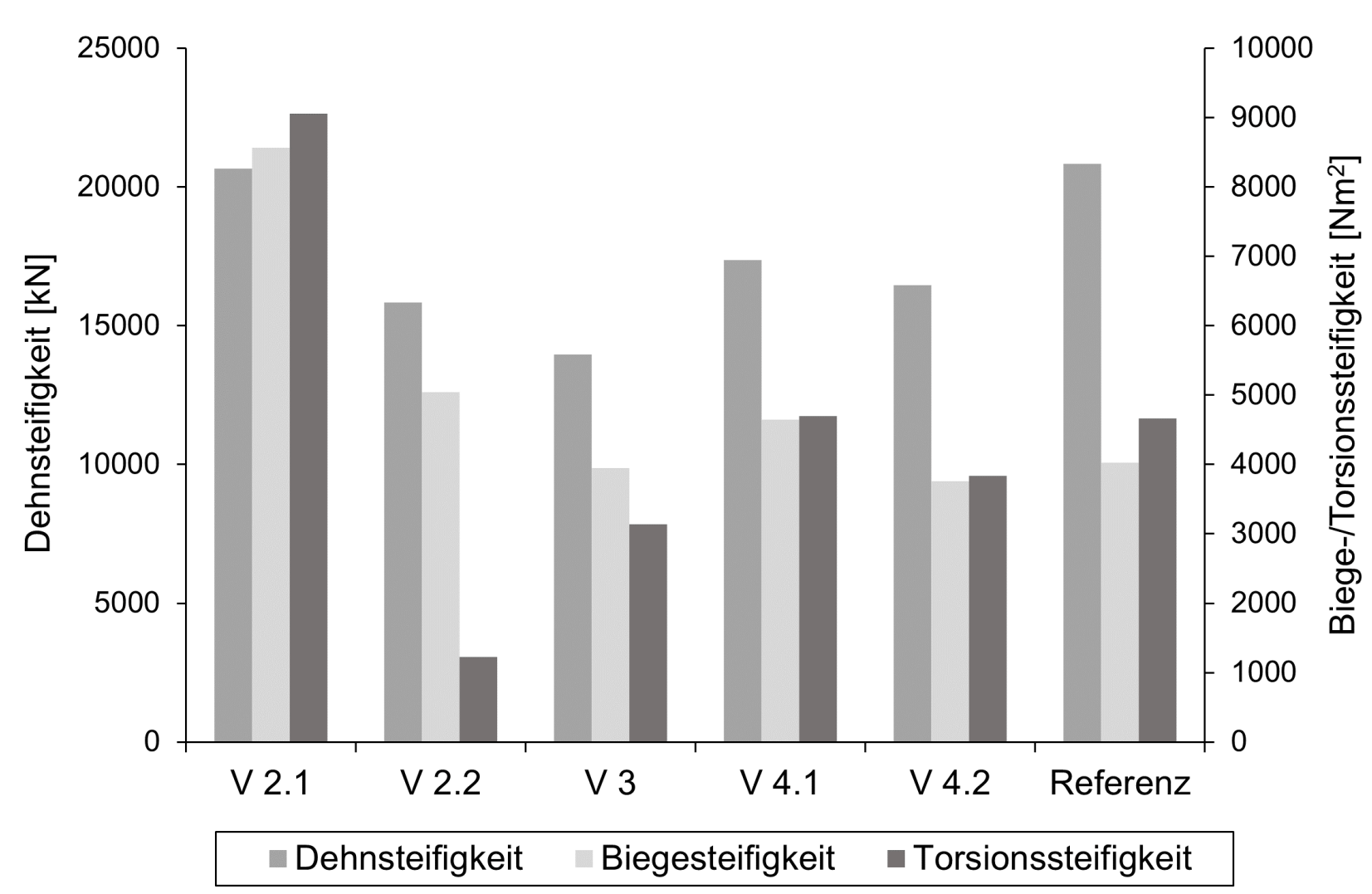

Abbildung 6-5: Dehn-, Biege- und Torsionssteifigkeiten der Varianten

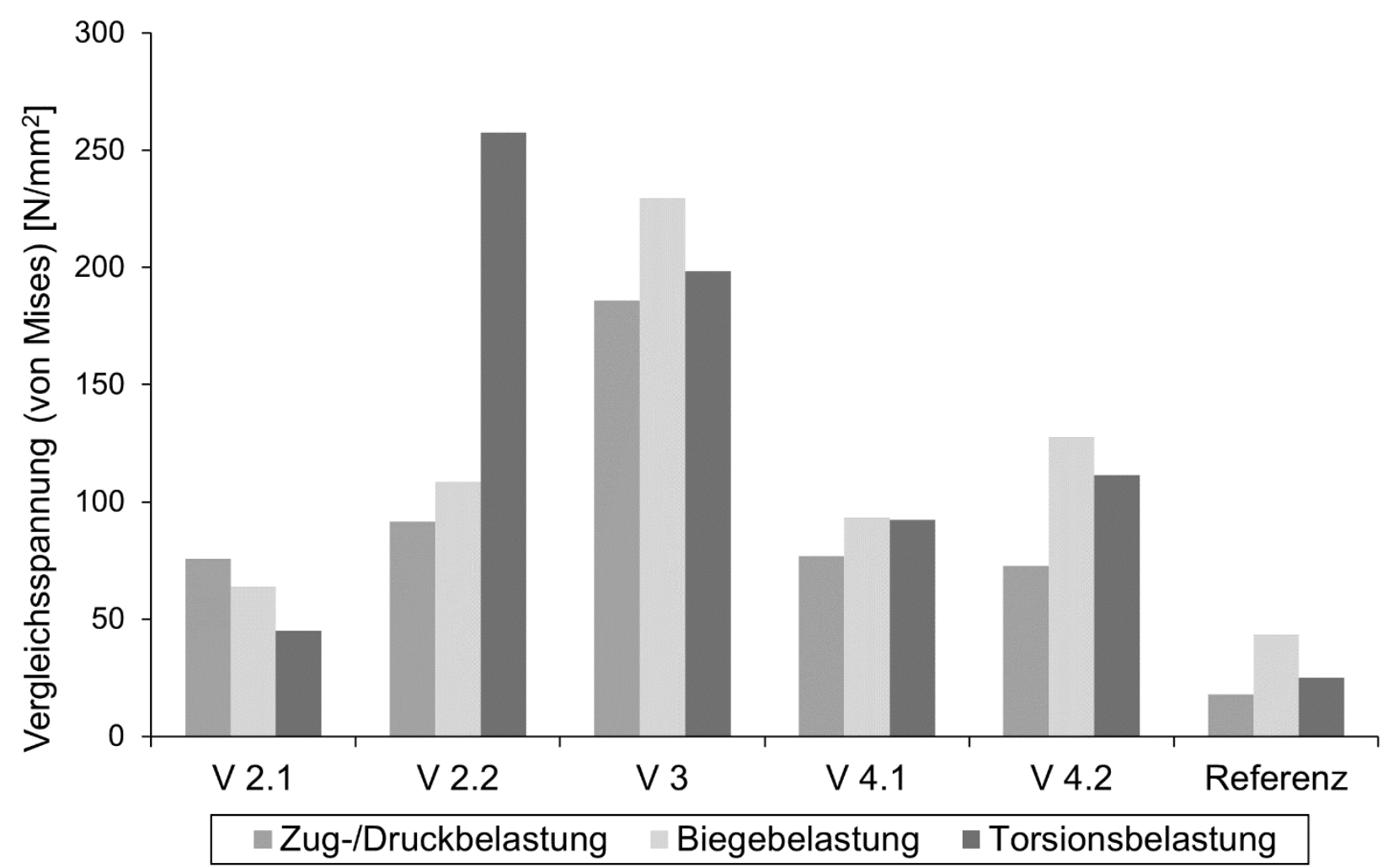

Abbildung 6-6: Maximale Vergleichsspannungen (von Mises) der Varianten 
Abbildung 6-6 trägt die Maximalwerte der ermittelten Vergleichsspannungen über den Varianten auf. Hier lässt sich erkennen, dass die Varianten 2.2 und 3 deutliche Spannungsüberhöhungen aufweisen, die, wenn auch nur lokal, durch eingebrachte Bauteilkerben verursacht werden. Um eine bessere Aussage über die Werkstoffausnutzung zu erhalten, werden die Spannungen aller Knoten des FEModells untersucht und ermittelt, welcher Anteil dieser Knoten eine Spannung oberhalb von $50 \%$ der Maximalspannung aufweist. Der so ermittelte Relativwert lässt erste Aussagen über eine vorteilhafte bzw. nachteilige Werkstoffausnutzung der jeweiligen Variante in Bezug auf ein Referenzrohr zu, welches nicht durch Innenhochdruckumformen umgeformt wurde (siehe Eingangsdaten in Anhang A). Die abschließende Bewertung der Varianten wurde mittels gewichteter Bewertungskriterien (Gewichtung siehe Anhang A) durchgeführt (siehe Tabelle 6-3).

Tabelle 6-3: Bewertung der Varianten mittels gewichteter Bewertungskriterien

\begin{tabular}{|c|c|c|c|c|c|c|c|c|c|c|c|c|}
\hline \multirow{2}{*}{\multicolumn{2}{|c|}{$\begin{array}{l}\text { Kriterium } \\
\text { Befestigung }\end{array}$}} & \multirow{2}{*}{$\begin{array}{l}\text { Gew. } \\
0,80\end{array}$} & \multicolumn{2}{|c|}{ V 2.1} & \multicolumn{2}{|c|}{ V 2.2} & \multicolumn{2}{|c|}{ V 3} & \multicolumn{2}{|c|}{ V 4.1} & \multicolumn{2}{|c|}{ V 4.2} \\
\hline & & & 0 & 0,00 & 1 & 0,80 & 1 & 0,80 & 2 & 1,60 & 2 & 1,60 \\
\hline Erreichbarke & & 0,60 & 3 & 1,80 & 3 & 1,80 & 3 & 1,80 & 2 & 1,20 & 2 & 1,20 \\
\hline \multirow{3}{*}{ Steifigkeit } & Zug/Druck & 0,33 & 4 & 1,33 & 3 & 1,00 & 3 & 1,00 & 4 & 1,33 & 3 & 1,00 \\
\hline & Biegung & 0,33 & 4 & 1,33 & 2 & 0,67 & 2 & 0,67 & 2 & 0,67 & 2 & 0,67 \\
\hline & Torsion & 0,33 & 4 & 1,33 & 0 & 0,00 & 1 & 0,33 & 2 & 0,67 & 2 & 0,67 \\
\hline \multirow{3}{*}{$\begin{array}{l}\text { Werkstoff- } \\
\text { ausnutzung }\end{array}$} & Zug/Druck & 0,20 & 0 & 0,00 & 0 & 0,00 & 0 & 0,00 & 0 & 0,00 & 0 & 0,00 \\
\hline & Biegung & 0,20 & 0 & 0,00 & 0 & 0,00 & 0 & 0,00 & 0 & 0,00 & 0 & 0,00 \\
\hline & Torsion & 0,20 & 0 & 0,00 & 0 & 0,00 & 0 & 0,00 & 0 & 0,00 & 0 & 0,00 \\
\hline \multicolumn{2}{|l|}{ Masse } & 0,50 & 0 & 0,00 & 0 & 0,00 & 2 & 1,00 & 2 & 1,00 & 1 & 0,50 \\
\hline \multicolumn{2}{|l|}{ Reinigbarkeit } & 0,10 & 3 & 0,30 & 3 & 0,30 & 2 & 0,20 & 2 & 0,20 & 2 & 0,20 \\
\hline \multicolumn{2}{|l|}{ Summe } & & 18 & 6,10 & 12 & 4,57 & 14 & 5,80 & 16 & 6,67 & 14 & 5,83 \\
\hline
\end{tabular}

Die Bewertung zeigt, dass alle Varianten deutliche Defizite in Bezug auf die Werkstoffausnutzung und Masse haben. Die schlechte Werkstoffausnutzung erklärt sich vor allem durch das Einbringen des Flaschenhalters als diskontinuierliches Formelement in den Fahrradrahmen. Aufgrund von Kerbwirkung an den Übergängen vom Formelement zum Rahmenrohr kommt es zu Spannungsüberhöhungen, die dazu führen, dass große Teile des Rohres eine im Vergleich zu diesen lokal auftretenden hohen Spannungen sehr geringe Spannungen erfahren. Eine entsprechend geringe Werkstoffausnutzung ist die Folge. Da alle Varianten eine ver- 
glichen mit dem Referenzrohr gleichermaßen geringe Werkstoffausnutzung aufweisen, hat dieses Kriterium einen vernachlässigbaren Einfluss auf die Entscheidung über die Wahl der favorisierten Varianten. Die Steifigkeit der Varianten ist durchweg positiv bewertet. Den Ausschlag für die Wahl der weiterzuverfolgenden Varianten geben letztlich die Kriterien Befestigung und Erreichbarkeit, die für die Funktionserfüllung des Flaschenhalters eine hohe Relevanz haben und damit eine entsprechend hohe Gewichtung erhalten.

\subsubsection{Ergreifen projektspezifischer Maßnahmen}

Im letzten Arbeitsschritt erfolgt das Integrieren der bewerteten Varianten in ein Gesamtkonzept. Dazu werden die aussichtsreichsten Varianten in den Fahrradrahmen eingefügt (siehe Abbildung 6-7). Die fertigungsintegrierenden Lösungen - im Sinne konkretisierter Gesamtkonzepte - sind in Abbildung 6-8 und Abbildung 6-9 abgebildet. Durch die Anwendung der zuvor beschriebenen Methodik wird sichergestellt, dass die Potenziale der Fertigungstechnologie bestmöglich ausgeschöpft werden. So beruhen die Lösungen nicht auf dem strikten Einhalten von Limitationen, um die Herstellbarkeit der Produkte zu gewährleisten, sondern darauf, neue Wege für die Konzipierung der Produkte im Kontext ihrer Funktion zu erschließen. Der hier ausgearbeitete Flaschenhalter stellt letztlich ein einfaches Beispiel dar, mittels dessen nur ein Bruchteil der fertigungstechnologischen Potenziale erschlossen wird. Der Grad der Ausschöpfung dieser Potenziale hängt vor allem davon ab, wie detailliert die fertigungsintegrierende DPM vorbereitet und ausgearbeitet wird.

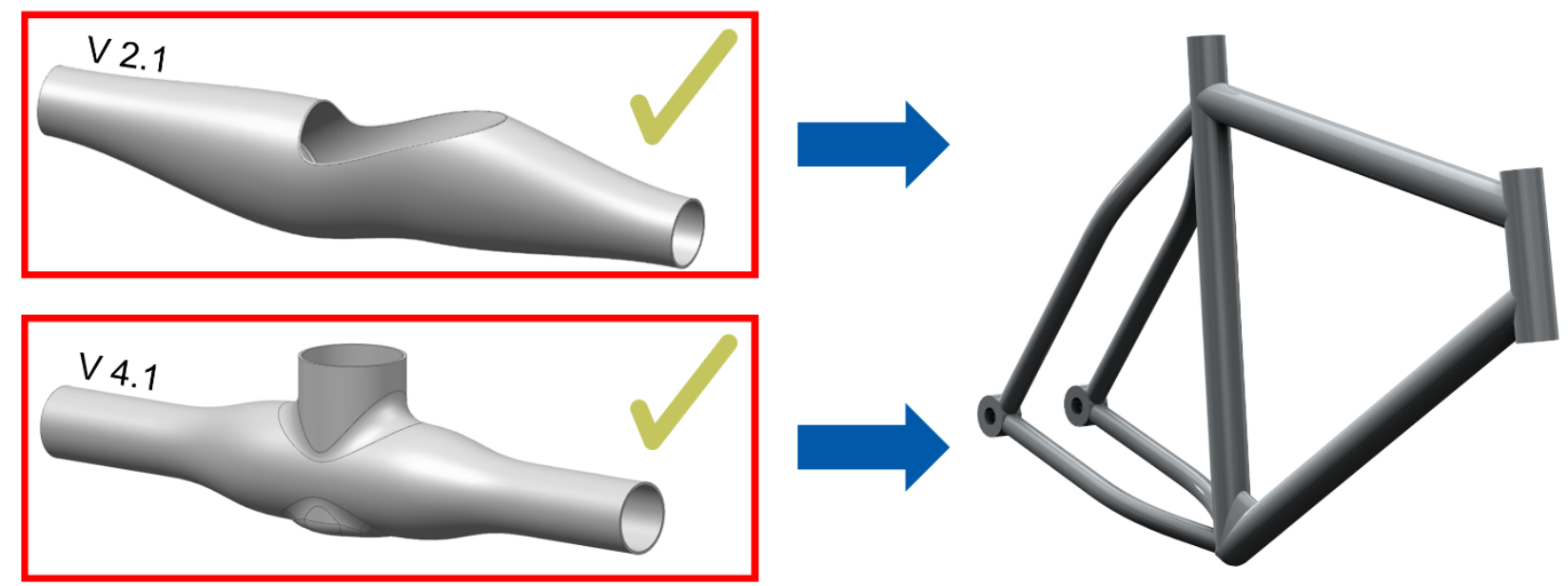

Abbildung 6-7: Projektspezifische Integration der aussichtsreichsten Varianten in den Fahrradrahmen 


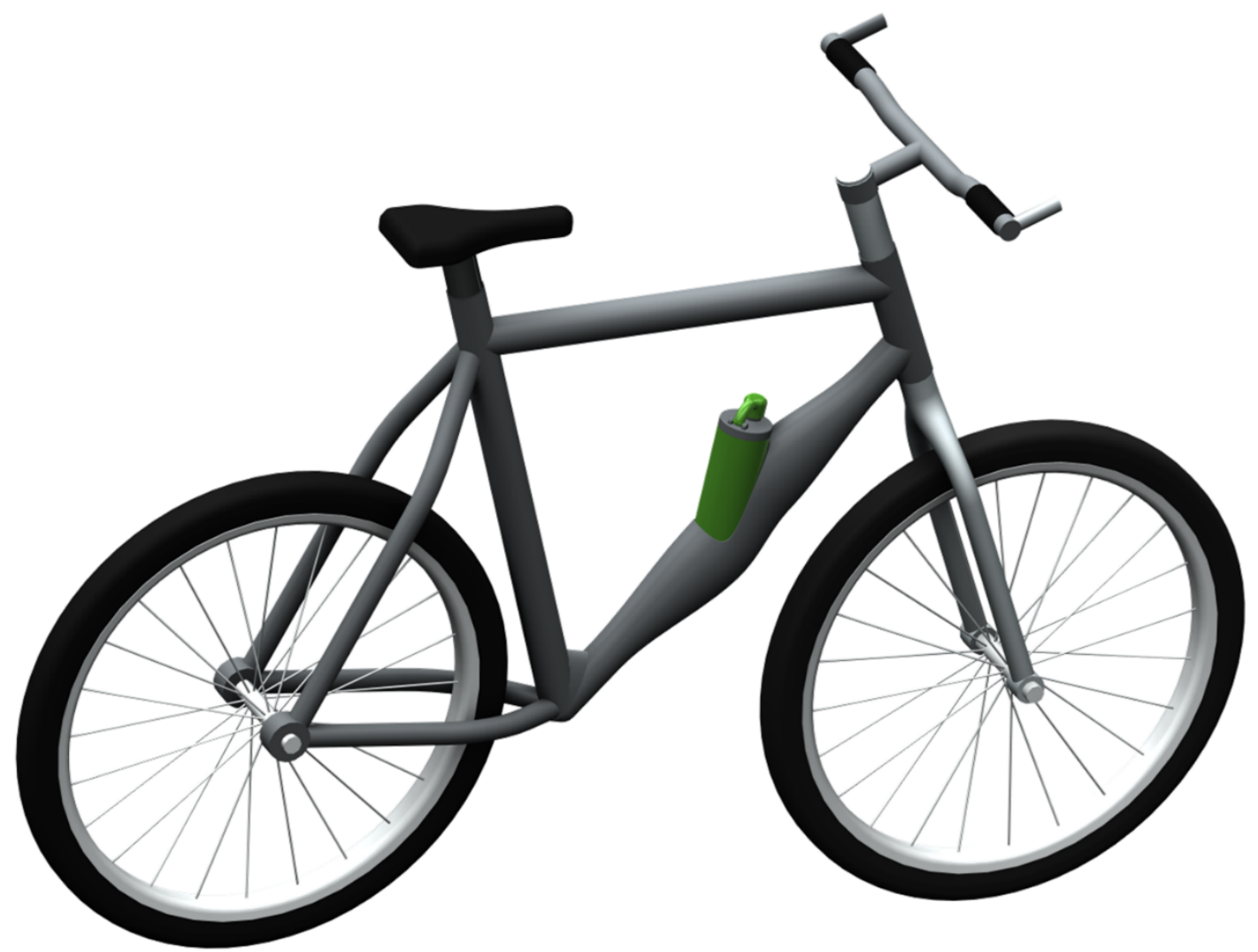

Abbildung 6-8: Fertigungsintegrierende Produktlösung 2.1 für einen innenhochdruckumgeformten Fahrradrahmen mit integriertem Flaschenhalter

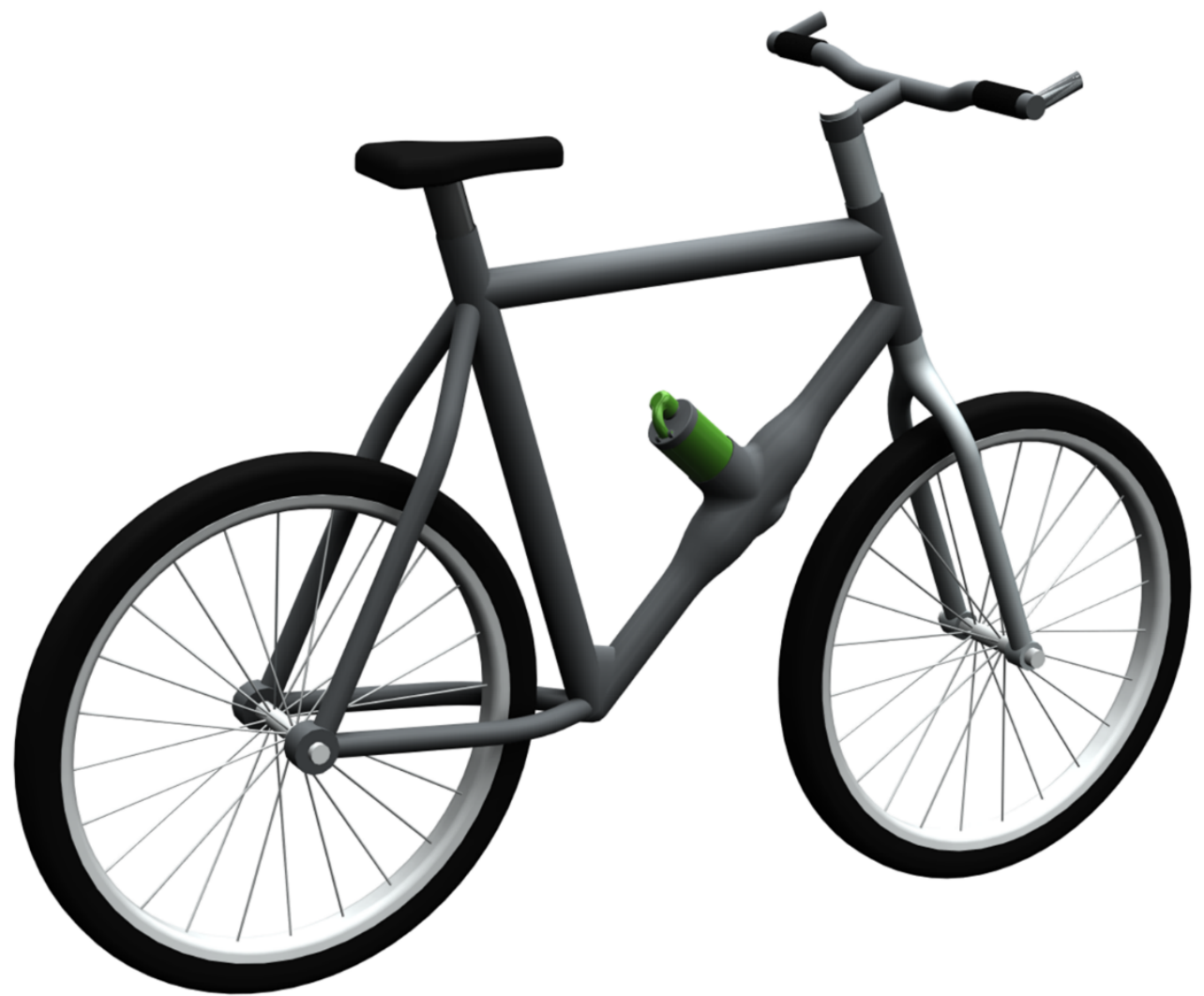

Abbildung 6-9: Fertigungsintegrierende Produktlösung 4.1 für einen innenhochdruckumgeformten Fahrradrahmen mit integriertem Flaschenhalter 


\subsection{Mehrfaches Antizipieren am Beispiel spaltprofilierter Deckenschienen}

Schienensysteme finden sich in vielen verschiedenen Anwendungsfeldern, wie beispielsweise in der Infrastruktur von Werkstätten. Dort werden sie eingesetzt, um schwere und unhandliche Lasten aufzuhängen und zwischen verschiedenen Orten zu transportieren. Für diese Deckenschienen sollen im Folgenden fertigungsintegrierende Produktlösungen entwickelt werden. Wie bereits Eingangs der Arbeit erwähnt, eignet sich insbesondere auch das Spaltprofilieren zur Herstellung von Führungen, die durch eine hohe Steifigkeit und Härte gekennzeichnet sind (siehe Abschnitt 1.1). ${ }^{246}$ Eine zusätzliche Herausforderung von Deckenschienensystemen ist das Anbringen und Lösen angehängter Lasten. Im Rahmen eines Entwicklungsprojekts sollen die Profile des Deckenschienensystems so konstruiert werden, dass ein einfaches reversibles Anbringen und Lösen externer Lasten möglich ist. Zu diesem Zweck wurde das Fügeverfahren „Federnd Einspreizen“247 als ein aussichtsreicher Ansatz zur Lösung dieser Problemstellung identifiziert. Ziel ist es, Lösungen für ein spaltprofiliertes Deckenschienensystem zu finden, in welches Verbindungselemente federnd eingespreizt (eingeschnappt ${ }^{248}$ ) werden können. Der Fokus bei der Entwicklung fertigungsintegrierender Produktlösungen liegt auf dem Ausschöpfen fertigungstechnologischer Potenziale für die Umsetzung profilintegrierter Schnappverbindungen (siehe Abbildung 6-10; Arbeitsschritt 1 der Methodik).
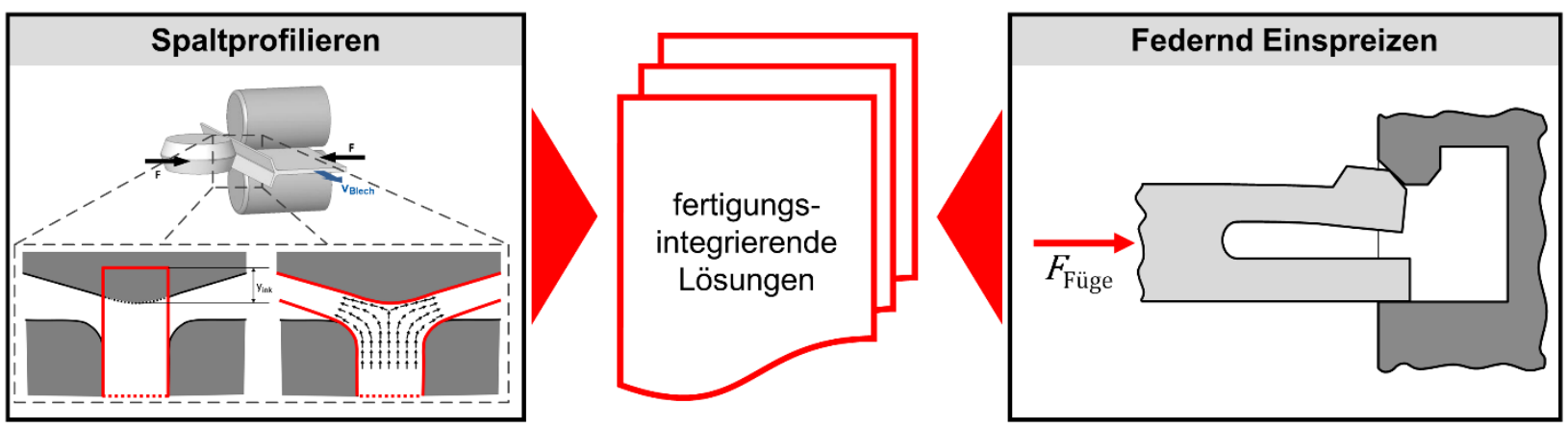

Abbildung 6-10: Erarbeitung fertigungsintegrierender Lösungen für spaltprofilierte Schnappverbindungen

${ }^{246}$ Vgl. Gramlich et al. (2015), S. 4 ff.

${ }^{247}$ Vgl. DIN 8593-1 (2003), S. 5.

${ }^{248}$ Im Rahmen des Fügeverfahrens Federnd Einspreizen werden meist Schnappverbindungen eingesetzt. Im weiteren Verlauf der Arbeit findet sich daher auch der Begriff Schnappen für dieses Fügeverfahren. 
Mit der Festlegung auf Schnappverbindungen sind auch die Fügeverfahren festgelegt, die im Rahmen der nachfolgenden Prozessanalysen genauer untersucht werden müssen (Arbeitsschritt 2 der Methodik). Damit steht jedoch auch fest, dass neben den Fertigungsprozessen auch die Fügeprozesse betrachtet und für die Lösungsfindung mitberücksichtigt werden müssen. Dazu ist es wichtig, die Fügeprozesse am Beispiel von Deckenschienen in den Produktlebenslauf einzuordnen. Abbildung 6-11 zeigt den Produktlebenslauf mit Fokus auf den Prozessen Fügen und Lösen, die im Rahmen der Produktnutzung vor Ort durchgeführt werden. Die Fügeprozesse (als Teil der Montageprozesse) sind somit als fertigungsanaloge Nutzungsprozesse anzusehen. Sie werden analog zu Fertigungsprozessen modelliert. Die folgenden Ausführungen beschränken sich auf diese Fügeprozesse. Die Löseprozesse können grundsätzlich analog dazu bearbeitet werden.

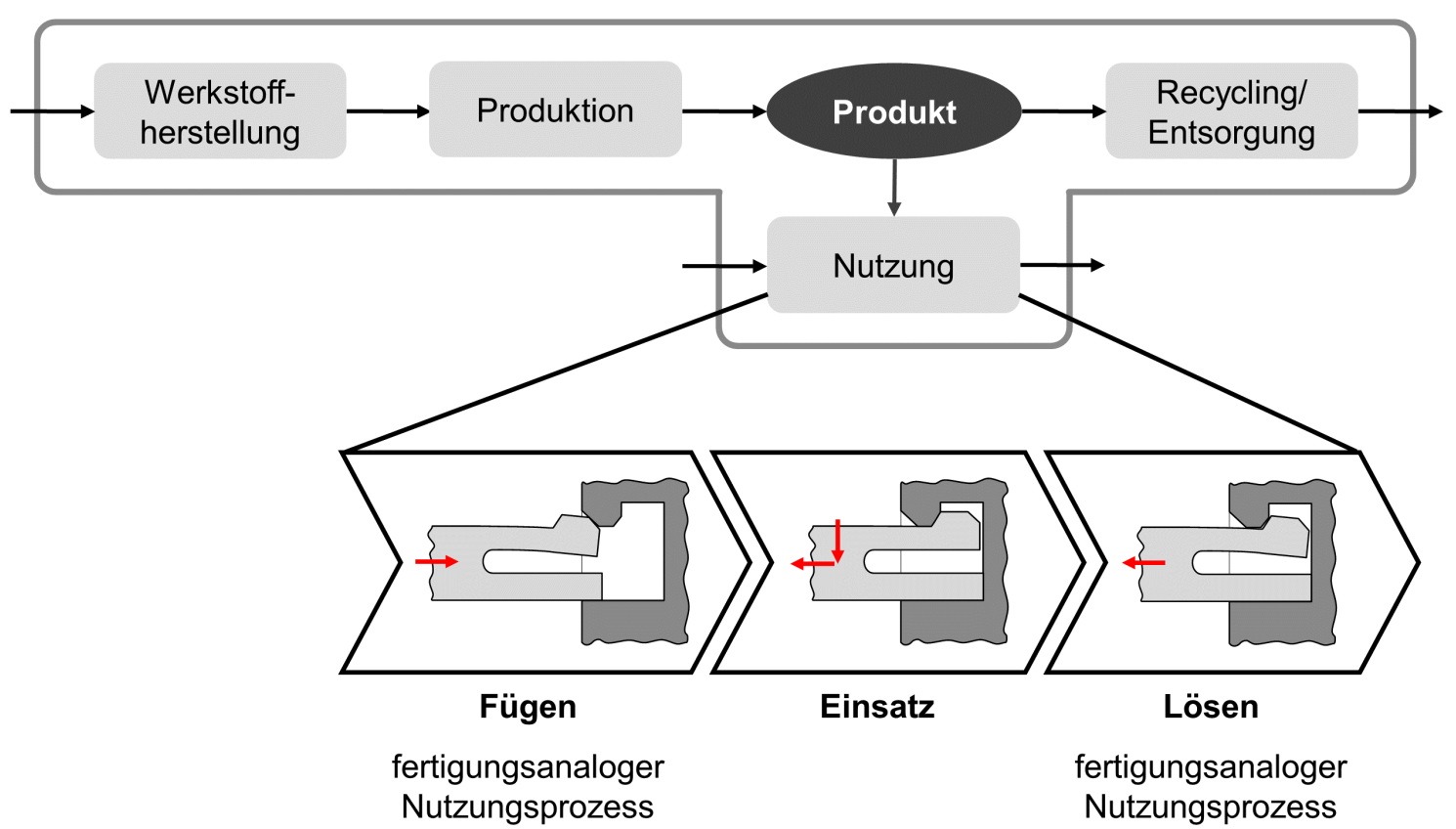

Abbildung 6-11: Einordnung der Füge- und Löseprozesse von Schnappverbindungen in
den Produktlebenslauf

Die bisherigen Ausführungen zeigen, dass für das konkrete Beispiel spaltprofilierter Schnappverbindungen nicht mehr die Funktionsträger des Produkts im Fokus der Entwicklung stehen, sondern die Elemente, die für die Durchführung des Fügeprozesses unabdingbar sind. Gemäß Kapitel 4 sind das die verfahrensprinziprelevanten Wirkelemente des Fügeverfahrens. Das Ziel der Anwendung der Methodik aus Kapitel 5 ist daher die Erarbeitung fertigungsintegrierender Produktlösungen, die die Fertigungspotenziale im Hinblick auf die Fügeprozesse spaltprofi- 
lierter Schnappverbindungen ausschöpfen. Daher müssen neben den Fertigungsprozessen auch die Fügeprozesse antizipiert werden, sodass gezielt Lösungselemente für die Schnappverbindung erarbeitet werden können. Abbildung 6-12 zeigt das angepasste Vorgehen, dass das mehrfache simultane Antizipieren von Fertigungs- und Fügeprozessen abbildet. Gemäß diesem Vorgehen wird zuerst das Spaltprofilieren unter Verwendung des VP-Modells analysiert, wie dies bereits am Beispiel des Innenhochdruckumformens gezeigt wurde. Die dabei ermittelten verfahrensinduzierten Gestaltelemente und ihre Eigenschaften sind bereits in Abbildung 4-16 dargestellt. Damit sind die Arbeitsschritte 3 und 4 der Methodik fertigungsseitig abgeschlossen. Im nächsten Schritt müssen die Fügeprozesse antizipiert werden.

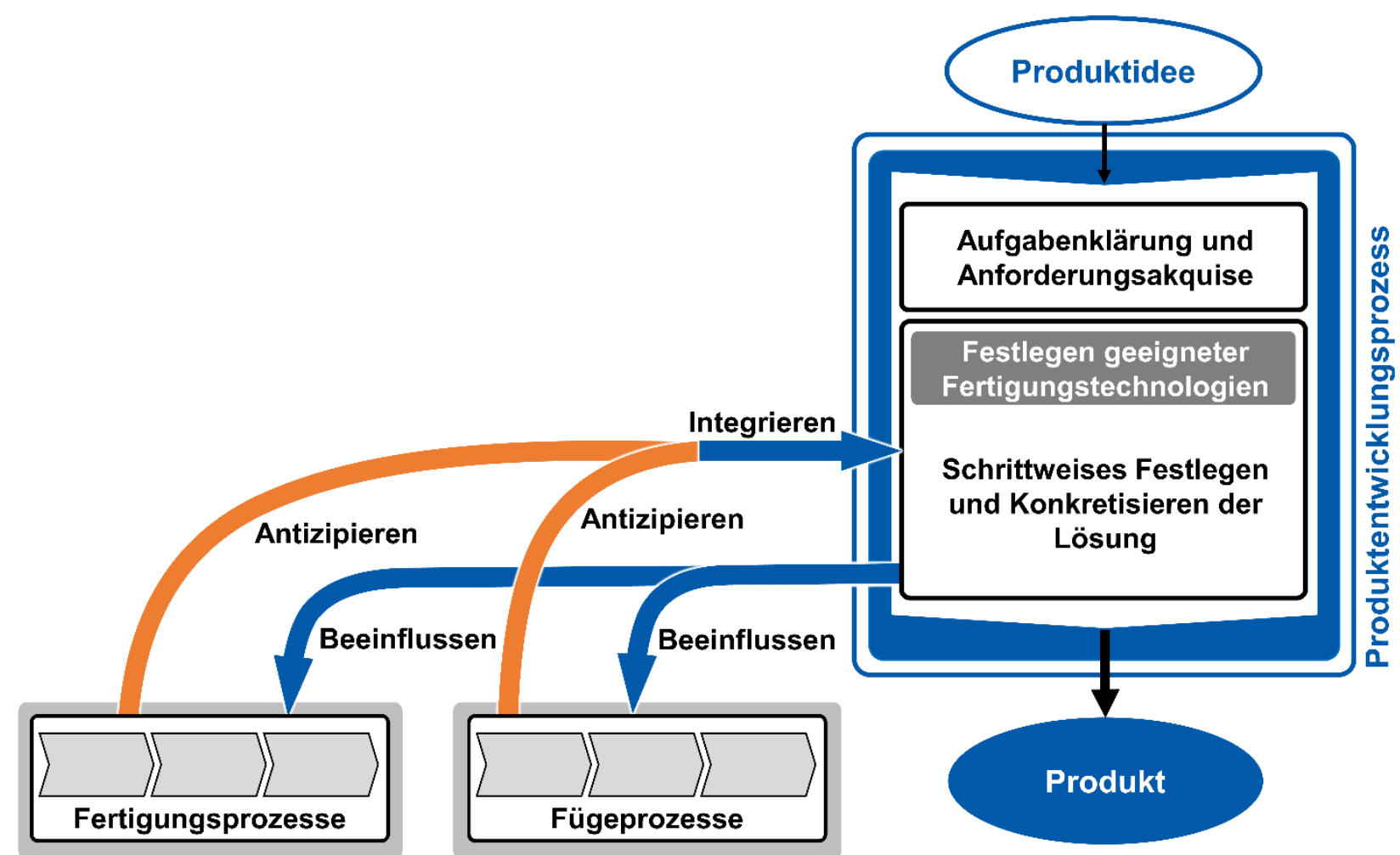

Abbildung 6-12: Vorgehen zum simultanen Antizipieren von Fertigungsprozessen und Fügeprozessen

\subsubsection{Antizipieren des Fügens von Schnappverbindungen}

Analog zum Antizipieren der Fertigungsprozesse müssen auch die Fügeprozesse gemäß den Arbeitsschritten 3 und 4 der Methodik analysiert werden. Abbildung 6-13 zeigt dazu das Verfahrensprinzip für das Schnappen. Dabei wird deutlich, dass der Fügeprozess in mehrere Teilprozesse eingeteilt werden kann, die durch drei Zustände charakterisiert sind. Im Zustand I kontaktieren sich Schnapphaken 
und Gegenkörper bedingt durch die Fügekraft $F_{\text {Füge }}$ an der schrägen Stirnkante (Wirkflächenpaar ${ }^{249}$ ), über die die Querkraft $Q$ übertragen wird. Diese ist im weiteren Verlauf verantwortlich für die Auslenkung des Schnapphakens (Zustand II), dessen Steifigkeit vor allem durch das rot markierte Wirkelement dominiert wird. In seiner maximal ausgelenkten Position kontaktiert der Schnapphaken den Gegenkörper über ein Wirkflächenpaar, das nicht mit dem Wirkflächenpaar in Zustand I identisch ist. Im Zustand III berühren sich Schnapphaken (samt zusätzlichem Führungselement) und Gegenkörper an zwei Wirkflächenpaaren in axialer Richtung. Es liegt somit ein Formschluss beider Fügepartner vor, der durch den steifigkeitsbedingten Widerstand des Schnapphakens erreicht wird.

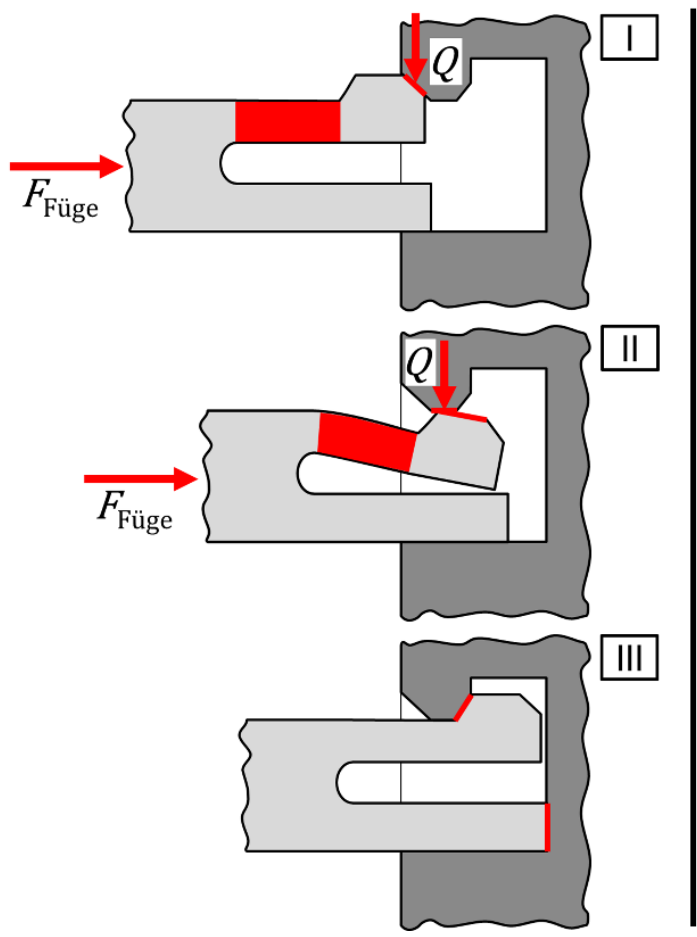

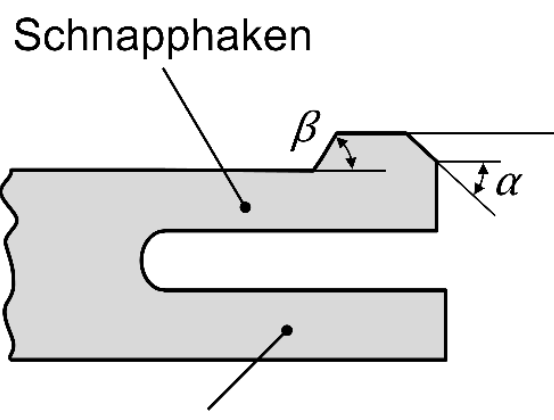

Führungselement

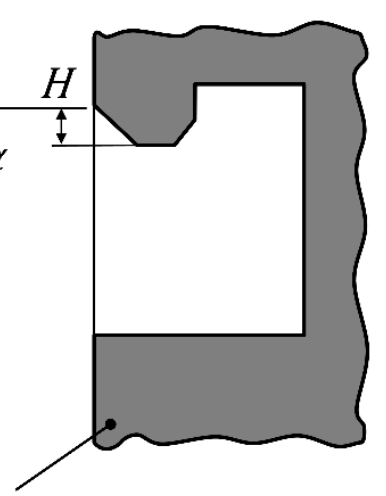

Gegenkörper

$H \quad$ Schnapphöhe (Hinterschnitt)

$\alpha \quad$ Fügewinkel

$\beta \quad$ Haltewinkel

$\mu \quad$ Reibungskoeffizient

Abbildung 6-13: Verfahrensprinzip des Schnappens (Federnd Einspreizen) unter Angabe der Zustände I bis III während des Fügevorgangs ${ }^{250}$

Das Verfahrensprinzip des Schnappens ist durch verschiedene Wirkzusammenhänge charakterisiert. Insbesondere ist das Zustandekommen der Fügekraft $F_{\text {Füge }}$ herauszustellen, da diese für die Auslegung der Verbindung eine wesentliche

${ }^{249}$ Wirkflächen dienen in erster Linie der Übertragung von Wirkgrößen (Energien und Signale).

Die Wirkfläche steht dabei meist in Interaktion mit einer zweiten Wirkfläche. Eine solche Kombination wird als Wirkflächenpaar bezeichnet. (Vgl. Feldhusen, Grote (2013), S. 480 f.)

250 In Anlehnung an Gramlich (2013), S. 112 und DVS 2242-1 (2011). 
Rolle spielt. Die Fügekraft bestimmt, wie hoch der Aufwand für den Nutzer während der Montage der Verbindung ist. Abbildung 6-14 nutzt das VP-Modell, um die wesentlichen Wirkelemente und Wirkgrößen herauszuarbeiten, die die Fügekraft $F_{\text {Füge }}$ beeinflussen. Diese sind für die nachfolgende Lösungsfindung zu berücksichtigen. Sowohl der Schnapphaken samt den Führungselementen als auch der Gegenkörper sind Operanden des Fügeprozesses. Da das Fügen mittels Handbetätigung durch den Nutzer ausgeführt werden soll, ist außer den bekannten Wirkgrößen kein separates Arbeitsmittel aufzuführen.

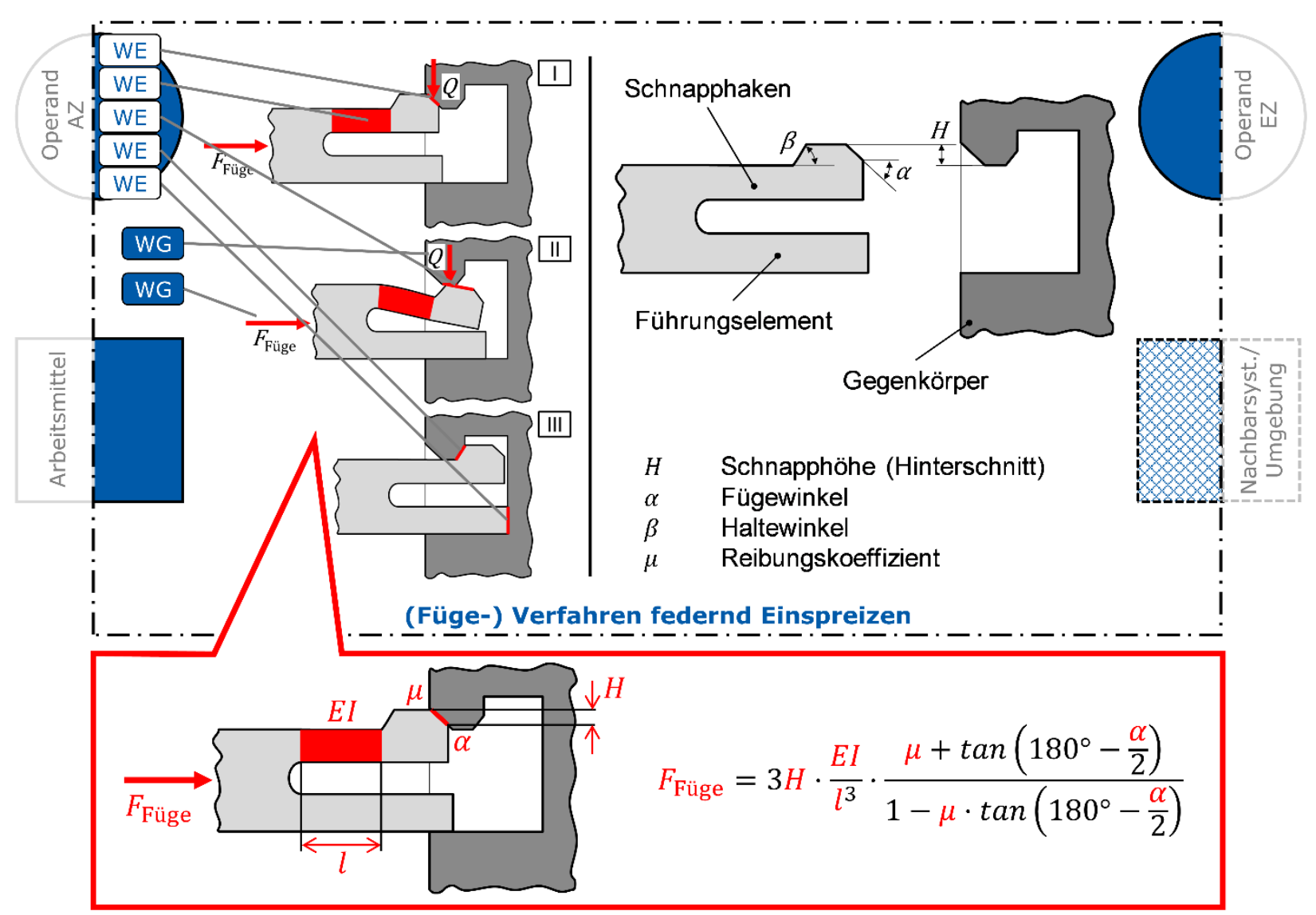

Abbildung 6-14: Antizipieren des Fügeprozesses auf Basis des Verfahrens Schnappen ${ }^{251}$

\subsubsection{Aufbereiten der Ergebnisse aus dem Antizipieren}

Die vorherigen Ausführungen zeigen, dass die Lösungsfindung für Produkte, die durch Fügeprozesse dominiert werden, im Wesentlichen auf den verfahrensprinziprelevanten Wirkelementen des Fügeverfahrens aufbauen muss. Die fertigungsintegrierende DPM wird daher so aufgebaut, dass die verfahrensprinziprelevanten Wirkelemente des Fügeverfahrens in den Zeilenköpfen stehen. Die Spaltenköpfe

${ }^{251}$ Formelzusammenhänge in Anlehnung an Bauer, Althof (1991), S. $307 \mathrm{ff}$. 
werden nach wie vor durch die verfahrensinduzierten Gestaltelemente bestimmt (siehe Abbildung 6-15). Für das hier gezeigte Beispiel resultieren die verfahrensinduzierten Gestaltelemente aus dem Spaltprofilieren sowie aus nachgelagerten Fertigungsverfahren, die im Rahmen einer kontinuierlichen Fließfertigung eingesetzt werden können. ${ }^{252}$ Auf Basis der DPM werden verschiedene fertigungsintegrierende Design Patterns für spaltprofilierte Schnappverbindungen erarbeitet. Abbildung 6-15 zeigt ein Design Pattern, in dem der spaltprofilierte Flansch als Schnapphaken genutzt wird. Die zugehörige Formel drückt die Eigenschaftszusammenhänge aus, die die Fügekraft und damit den Kernaspekt des Design Patterns charakterisieren. Diese gehen zurück auf die Wirkzusammenhänge, die die verfahrensprinziprelevanten Wirkelemente im Kontext des Fügeverfahrens charakterisieren. Die verfahrensinduzierten Eigenschaften bzw. deren Einfluss auf die Fügekraft $F_{\text {Füge }}$ sind rot markiert. Damit ist auch der Arbeitsschritt 5 der Methodik abgeschlossen.

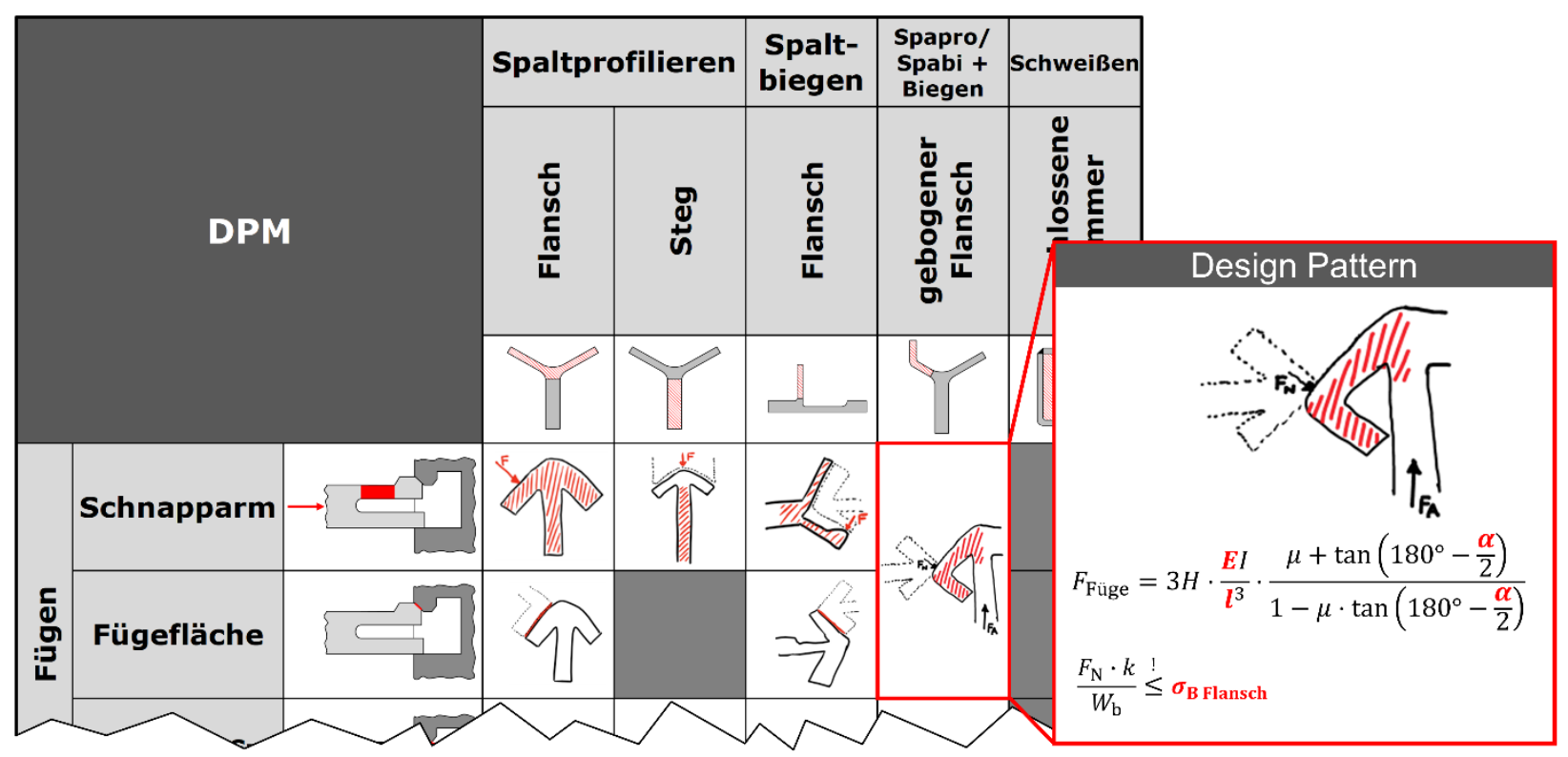

Abbildung 6-15: Design Pattern Matrix für spaltprofilierte Schnappverbindungen ${ }^{253}$

\subsubsection{Ergreifen projektspezifischer Maßnahmen}

Im letzten Arbeitsschritt (Arbeitsschritt 6 der Methodik) werden auf Basis der allgemeingültigen und in Form von Design Patterns dokumentierten Lösungsele- 
mente projektspezifische Maßnahmen abgeleitet. Für die spaltprofilierte Schnappverbindung bedeutet das, dass die dargestellten Lösungselemente in die Lösungsfindung für das spaltprofilierte Deckenschienensystem einbezogen werden. Ein wesentlicher Vorteil der verwendeten Methodik steckt u. a. darin, dass die Lösungsfindung im Rahmen des Produktkonkretisierungsprozesses nicht an ein bestimmtes Vorgehensmodell gebunden sein muss. So können insbesondere für Problemstellungen mit komplexen Randbedingungen auch algorithmenbasierte Ansätze zur Lösungsfindung verwendet werden. Am Beispiel von Schnappverbindungen sind vor allem die konfliktären Anforderungen zwischen Füge- und Einsatzprozess ausschlaggebend für die Verwendung eines solchen Vorgehens. ${ }^{254}$ Während die Fügekraft möglichst niedrig sein sollte, sollte demgegenüber die Haltekraft während des Einsatzprozesses möglichst hoch sein. Die Haltekraft der Schnappverbindung ist für die Tragfähigkeit des Deckenschienensystems ebenso wichtig wie die Steifigkeit des Deckenschienenprofils. Die Schwierigkeit bei der Auslegung der Schnappverbindung liegt darin, dass Füge- und Haltekräfte signifikant von der Steifigkeit des Schnapphakens abhängen. Ist diese zu hoch gewählt, können zwar höhere Haltekräfte realisiert werden. Die Fügekräfte nehmen jedoch ebenfalls zu. Zusätzlich können die Kräfte auch über den Winkel der einzelnen Wirkflächenpaare beeinflusst werden, die im jeweiligen Prozess im Eingriff sind. Die Geometrie der Schnappverbindung sowie die des Deckenschienenprofils müssen daher so festgelegt werden, dass ein möglichst optimales Verhältnis aus Füge- und Haltekraft erreicht wird. Die Erarbeitung einer Lösung unter Verwendung eines analytischen Vorgehens scheint daher kaum möglich. Es bietet sich die Anwendung eines algorithmenbasierten Ansatzes an, bei dem die Lösungsgenerierung und die Bewertung der Lösung in einem Vorgang abgearbeitet werden.

Die algorithmenbasierte Lösungsfindung ist schematisch in Abbildung 6-16 dargestellt. Beginnend bei der Beschreibung der Menge aller denkbaren Lösungen (z. B. durch Produkteigenschaften) werden Nebenbedingungen aufgestellt, die den eigentlichen Lösungsraum beschränken. Am Beispiel des Deckenschienensystems sind das z. B. maximale Flanschlängen, die aus der Fertigung resultieren, maximale Fügekräfte und minimale Lösekräfte. Durch Angabe von Zielfunktionen wird die Suchrichtung festgelegt, in der die optimale Lösung im Lösungsraum zu finden ist. Für die Schnappverbindung resultiert die Zielfunktion aus der Forderung nach einer möglichst hohen Steifigkeit des Deckenschienenprofils, um die notwendigen

${ }^{254}$ Vgl. Lüthen et al. (2017), S. $147 \mathrm{ff.}$ 
Haltekräfte im Einsatzprozess zu realisieren. Die fertigungsintegrierenden Design Patterns sorgen dafür, dass der zuvor beschriebene Lösungsraum um zusätzliche Lösungen erweitert wird, die ohne Berücksichtigung fertigungstechnologischen Wissens nicht denkbar gewesen wären.

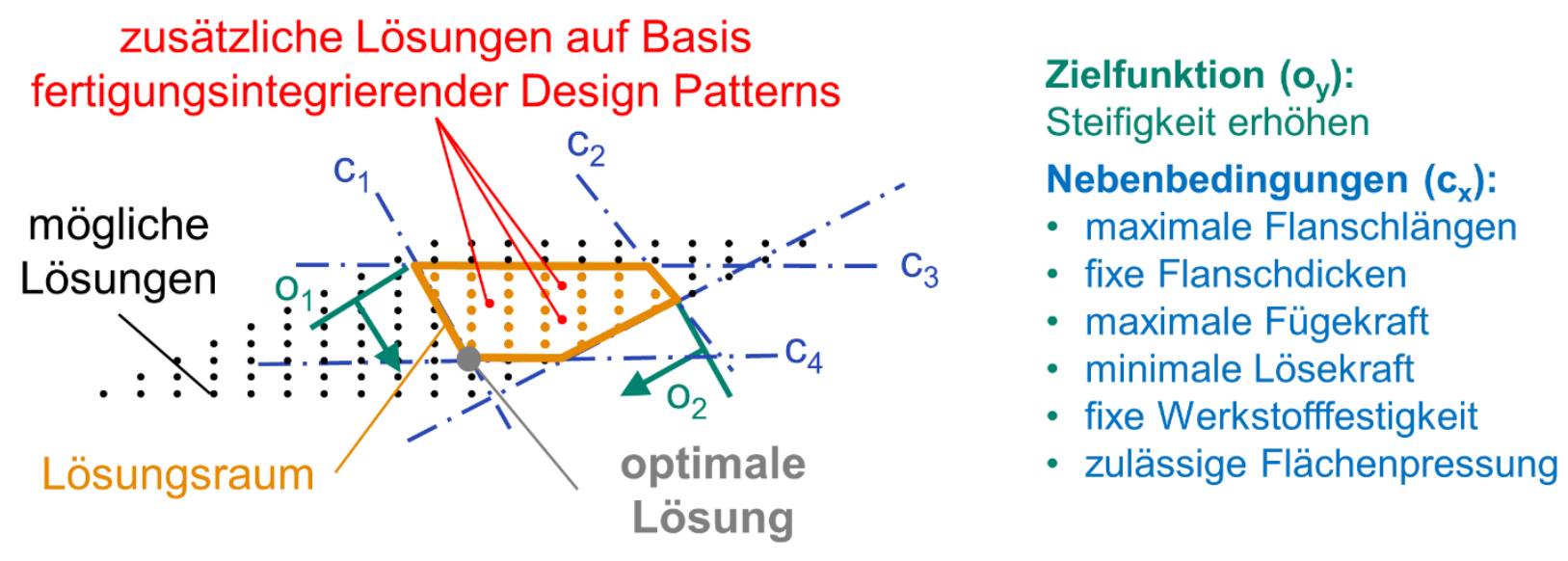

Abbildung 6-16: Algorithmenbasierte Lösungsfindung ${ }^{255}$

Um die Design Patterns für diese stark formalisierte Form der Lösungsfindung verwenden zu können, bedarf es einer entsprechenden Formalisierung der Entwicklungsaufgabe (siehe Abbildung 6-17). Dabei werden aus den projektspezifischen Anforderungen Zielfunktion(en) und Nebenbedingungen abgeleitet. Die unabhängigen Produkteigenschaften werden als Optimierungsvariablen verwendet. Die Design Patterns selbst fließen wiederum in ein Bauraumdiagramm ein, das die Startlösung für die algorithmenbasierte Lösungsfindung beinhaltet. ${ }^{256}$

Das in Abbildung 6-17 dargestellte Bauraumdiagramm enthält alle Angaben, die für die Modellierung einer zweidimensionalen Startlösung für die Optimierung notwendig sind. Das beinhaltet die Wirkflächen, über die die Kräfte zwischen Deckenschiene und Verbindungselement übertragen werden, die Wirkfläche, über die eine äußere Kraft eingeleitet wird, und die Wirkfläche, über die diese Kraft wieder ausgeleitet wird. Durch die Angabe von Bauraumbegrenzungen wird der Raum definiert, innerhalb dessen der Algorithmus die Lösung festlegen darf. Die Angabe konkreter Drehfreiheitsgrade reduziert die Komplexität der Lösungsfindung und damit den späteren Rechenaufwand, indem die Möglichkeiten der Formgebung auf gerade Profilelemente und definierte Abknickpunkte beschränkt wird. Der verwendete Algorithmus ist in der Lage, im Rahmen einer Formoptimierung sowohl

255 In Anlehnung an Birkhofer (2005), S. 52; Birkhofer, Wäldele (2005).

${ }^{256} \mathrm{Vgl}$. Lüthen et al. (2017), S. $163 \mathrm{ff}$. 
die Geometrie der Deckenschiene und des Verbindungselements als auch den Bauteilkontakt zu optimieren. Er sucht dazu nach lokalen Optima für die Zielfunktion.

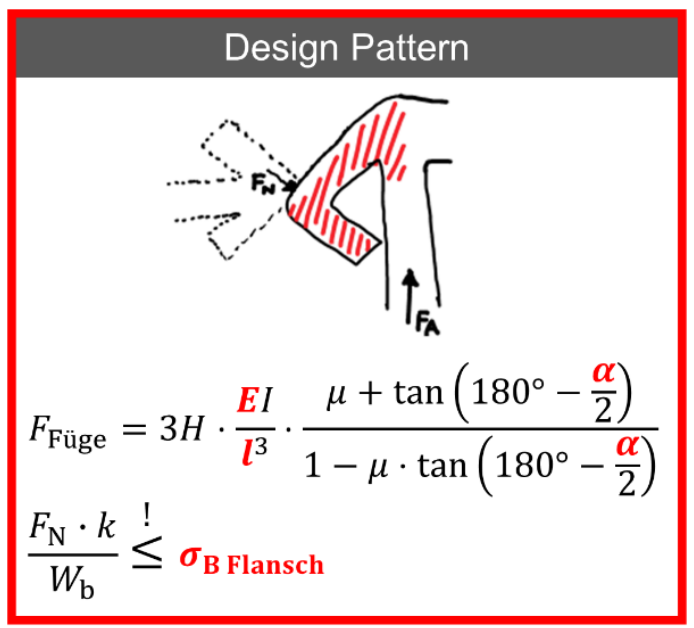

\section{Optimierungsvariablen}

\section{Nebenbedingungen}

Zielfunktion(en)

Bauraumdiagramm

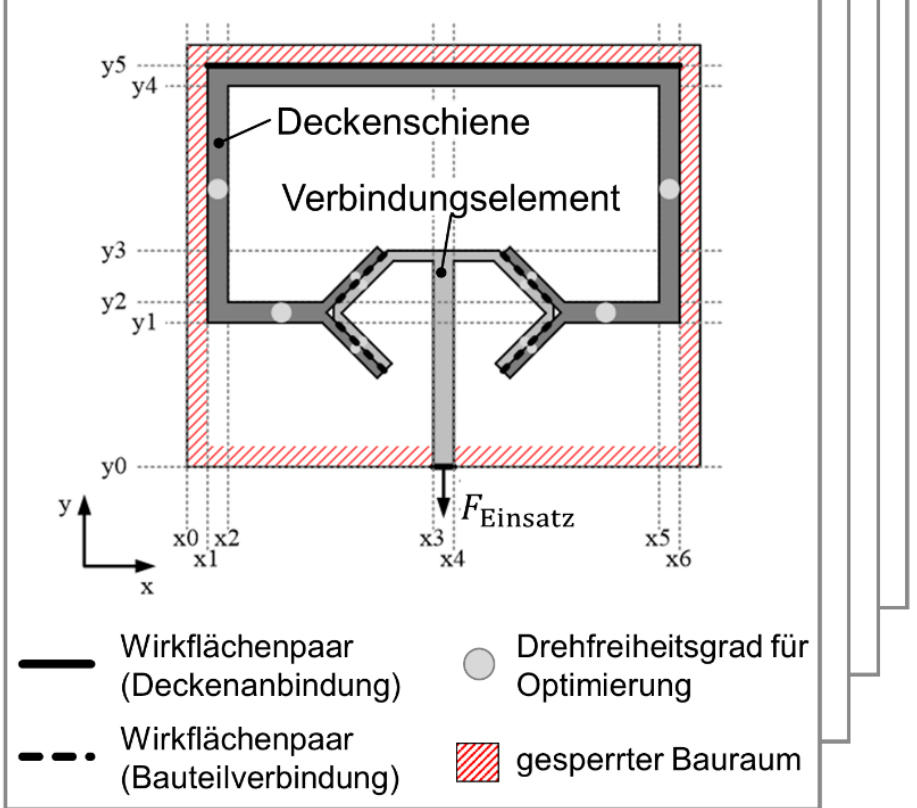

Abbildung 6-17: Formalisieren der Entwicklungsaufgabe ${ }^{257}$

Die Anwendung des Algorithmus liefert eine steifigkeitsoptimale Lösung für das spaltprofilierte Deckenschienensystem samt integrierter Schnappverbindung (Abbildung 6-18). ${ }^{258}$ Die sogenannte Compliance, ein Maß für die Minimierung der Zielfunktion, konnte im Vergleich zur Startlösung um 99,14 \% reduziert werden. Die Lösung zeichnet sich somit durch eine deutlich erhöhte Steifigkeit aus. Mit einem Querschnitt von $36 \mathrm{~mm}$ Breite und $45 \mathrm{~mm}$ Höhe des Deckenschienenprofils und einer Länge des Verbindungselements von $20 \mathrm{~mm}$ (Material jeweils: HC 480 LA) können Haltekräfte von bis zu 1200 N (Startlösung: 500 N) aufgenommen werden. Demgegenüber sind für den Fügeprozess nur Kräfte von bis zu 300 N (Startlösung: $700 \mathrm{~N}$ ) notwendig. Diese Lösung genügt damit nicht nur den Anforderungen des Einsatzprozesses. Durch die Einbeziehung des zuvor erarbeiteten Design

\footnotetext{
257 In Anlehnung an Roos et al. (2016), S. 126; Roos et al. (2017c), S. 325 und Roos et al.
} (2017a), S. 17.

258 Eine detaillierte Charakterisierung dieser Lösung findet sich in Roos et al. (2017c), S. 324 f. 
Patterns nutzt die Produktlösung zugleich auch die Möglichkeiten der Fertigungstechnologie Spaltprofilieren im Hinblick auf die angedachten Fügeprozesse. Die Lösung wird somit den Einsatz- und Fügeprozessen gleichermaßen gerecht.

a)

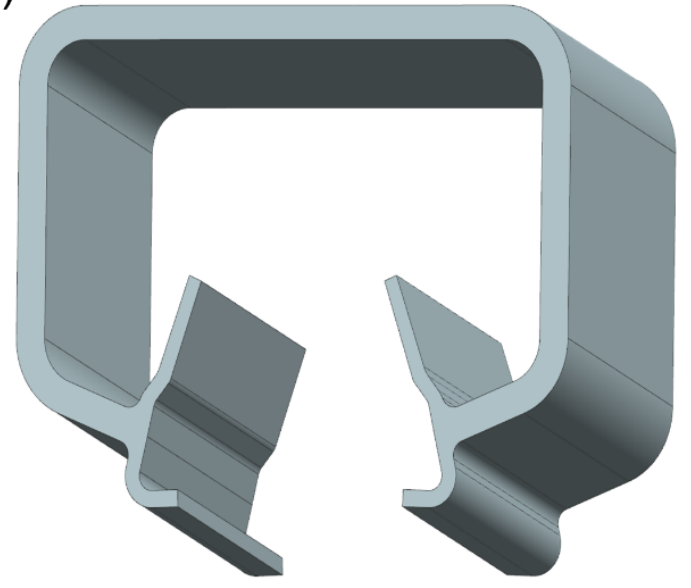

b)

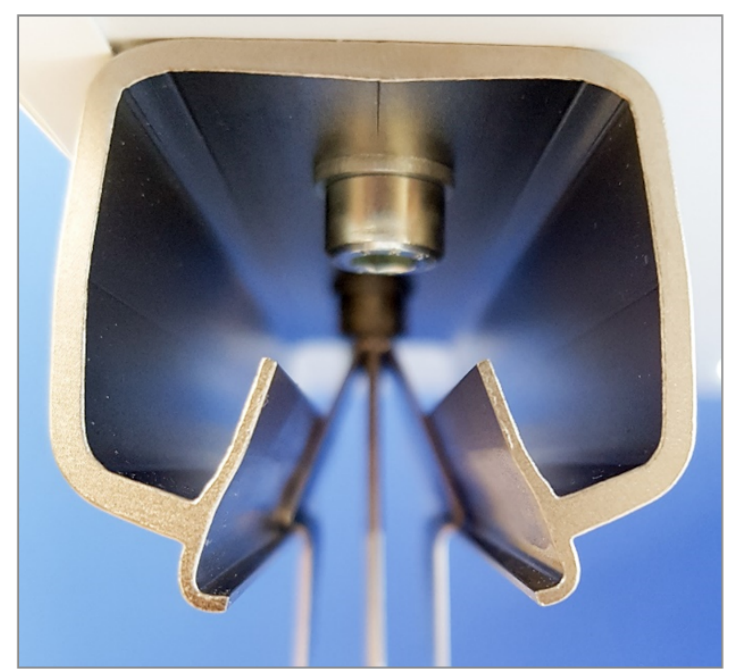

Abbildung 6-18: Optimierte Lösung für die spaltprofilierte Schnappverbindung als Teil eines Deckenschienensystems: a) CAD-Modell ${ }^{259}$; b) gefertigte Deckenschiene

Der wesentliche Vorteil, der sich aus der Anwendung der fertigungsintegrierenden Entwicklungsmethodik im Zusammenspiel mit einem algorithmenbasierten Entwicklungsansatzes ergibt, liegt darin, dass fertigungsintegrierende Produktlösungen auch unter komplexen Randbedingungen entwickelt werden können. Die Berücksichtigung von Fügeprozessen und die Generierung von Lösungen basierend auf deren verfahrensprinziprelevanter Wirkelemente stellen darüber hinaus einen wesentlichen Schritt in Richtung einer integrierten Produkt- und Prozessentwicklung dar. Ein Ansatz, der diesen Gedanken aufgreift, findet sich im Integrated Algorithm-Based Product and Process Development ${ }^{260}$. Vor allem das Antizipieren von Lebenslaufprozessen aus unterschiedlichen Lebenslaufphasen (Fertigung und Nutzung) bietet darin eine weit umfassendere Sicht auf das Produkt, als dies in konventionellen Entwicklungsansätzen der Fall ist. 


\section{Zusammenfassung und Ausblick}

Die Entwicklung fertigungsintegrierender Produktlösungen ist kein gänzlich neues Vorhaben. Erste Ansätze sind in Grundzügen bereits bei einer Vielzahl von Autoren zu finden. Dennoch zeigt diese Arbeit, dass ein solches Entwicklungsvorhaben nur dann zielgerichtet durchgeführt werden kann, wenn dem Entwickler ein systematisches Vorgehen zur Verfügung gestellt wird, mittels dessen fertigungstechnologisches Produkt-Wissen umfassend nutzbar gemacht wird. Die Modellierung von Verfahrensprinzipien im VP-Modell ermöglicht es, fertigungstechnologisches Produkt-Wissen möglichst umfassend abzubilden. Aufbauend auf den Ideen der GPPE und der produktionsintegrierenden Entwicklungsmethodik nach GRAMLICH wurde eine fertigungsintegrierende Entwicklungsmethodik erarbeitet, die eine schrittweise Aufarbeitung und Integration des fertigungstechnologischen ProduktWissens in den Produktentwicklungsprozess unterstützt. Auf Basis des frühzeitigen Antizipierens von Fertigungsprozessen mittels des VP-Modells und der darauf aufbauenden Integrationswerkzeuge, wie der DPM, kann fertigungstechnologisches Produkt-Wissen gezielt für die Produktentwicklung aufbereitet werden. Auf diese Weise werden neuartige Lösungsmöglichkeiten erschlossen, die das Potenzial der Fertigung umfassend ausschöpfen. Anhand der vorgestellten Evaluierungsbeispiele konnte gezeigt werden, dass mittels der fertigungsintegrierenden Entwicklungsmethodik auch im Zusammenhang mit unterschiedlichen Vorgehensmodellen ein konkreter Mehrwert für das Produkt generiert werden kann.

Im Rahmen dieser Arbeit wurde zudem ein Ansatz vorgestellt, der die Konkretisierung von Einsatzprozessen in Übereinstimmung mit der Produktkonkretisierung beschreibt. Das Resultat des Ansatzes ist eine technische Lösung, die nicht nur eine bestimmte Produktfunktion erfüllt, sondern auch den Einsatzprozessen bestmöglich gerecht wird. In den Evaluierungsbeispielen wurde darüber hinaus die Analogie von Fertigungsprozessen und fertigungsanalogen Nutzungsprozessen verwendet, um eine fertigungsintegrierende Produktlösung für Verbindungelemente zu erarbeiten. Die gemeinsame Berücksichtigung von Fertigungs-, Einsatzund fertigungsanalogen Nutzungsprozessen im Rahmen der Produktentwicklung ist ein erster grundlegender Schritt auf dem Weg hin zu einer gleichwertigen Produkt- und Prozessentwicklung, in der nicht nur das Produkt, sondern auch dessen Lebenslaufprozesse gleichermaßen konkretisiert werden. 
Die fertigungsintegrierende Entwicklungsmethodik muss in einem nächsten Schritt in der industriellen Praxis erprobt werden. Dies erfordert insbesondere die Abbildung von etablierten Schnittstellen zwischen Produktentwicklung und Fertigung in dem hier vorgestellten Ansatz. Für die Unterstützung des Antizipierens technischer Prozesse und die Erarbeitung fertigungstechnologischer Lösungselemente wurden Modelle, Methoden und Werkzeuge vorgestellt, deren konkrete Anwendung Erfahrung im Umgang mit derartigen Hilfsmitteln bedarf. Um die Anwendbarkeit in der Praxis sicherstellen zu können, müssen angepasste Formblätter, Workshopunterlagen, etc. zur Verfügung gestellt werden, mittels derer Projektteams gezielt in der Anwendung der vorgestellten Methodik unterstützt werden können. Aktuell fehlt es vor allem an kompakten Verfahrenssammlungen, die einen einfachen und umfassenden Überblick über das vorhandene fertigungstechnologische Wissen geben. Deren Bereitstellung ist eine wesentliche Grundlage, um sowohl Fertiger als auch Produktentwickler in ihrer Arbeit zu unterstützen. Die in dieser Arbeit vorgestellten Modelle bieten jedoch eine geeignete Basis, um das fertigungstechnologische Wissen strukturiert in entsprechenden Sammlungen oder Datenbanken zu dokumentieren.

Die Design Pattern Matrix wurde im Rahmen dieser Arbeit verwendet, um fertigungsintegrierende Lösungen zu erarbeiten. Ihr auf der Gegenüberstellung von Eigenschaften basierender Aufbau bietet die Möglichkeit, fertigungstechnologische Lösungen strukturiert abzulegen. Ähnlich dem Aufbau von Konstruktionskatalogen, wie sie beispielweise bei RoTH ${ }^{261}$ zu finden sind, könnten die fertigungsintegrierenden Lösungen somit eindeutig gegliedert in Katalogen abgelegt werden. Ein zusätzlicher Zugriffsteil böte die Möglichkeit, weitere Angaben zu den fertigungsintegrierenden Lösungen bereitzustellen, die deren Verwendung im Rahmen konkreter Entwicklungsprojekte unterstützen. Die Erarbeitung solcher Lösungskataloge scheint umso wichtiger, wenn auch Einsatzprozesse stärker in die Lösungsfindung einbezogen werden. Weiterer Forschungsbedarf besteht vor allem darin, die Interdependenzen zwischen Einsatz- und Fertigungsprozessen zu untersuchen, sodass diese frühzeitig im Entwicklungsprozess adressiert werden können.

${ }^{261}$ Konstruktionskataloge unterscheiden sich insofern von einfachen Lösungssammlungen, als dass sie insbesondere im Hinblick auf Vollständigkeit und Gliederung einen besseren Zugriff auf verfügbare Lösungen erlauben, die insbesondere auf die Tätigkeiten beim Konstruieren zugeschnitten sind. (Vgl. Roth (2001), S. 1.) 
Anhang A - Design Pattern Matrix und Punktbewertung für das Beispiel innenhochdruckumgeformter Fahrradrahmen

A1 - Design Pattern Matrix

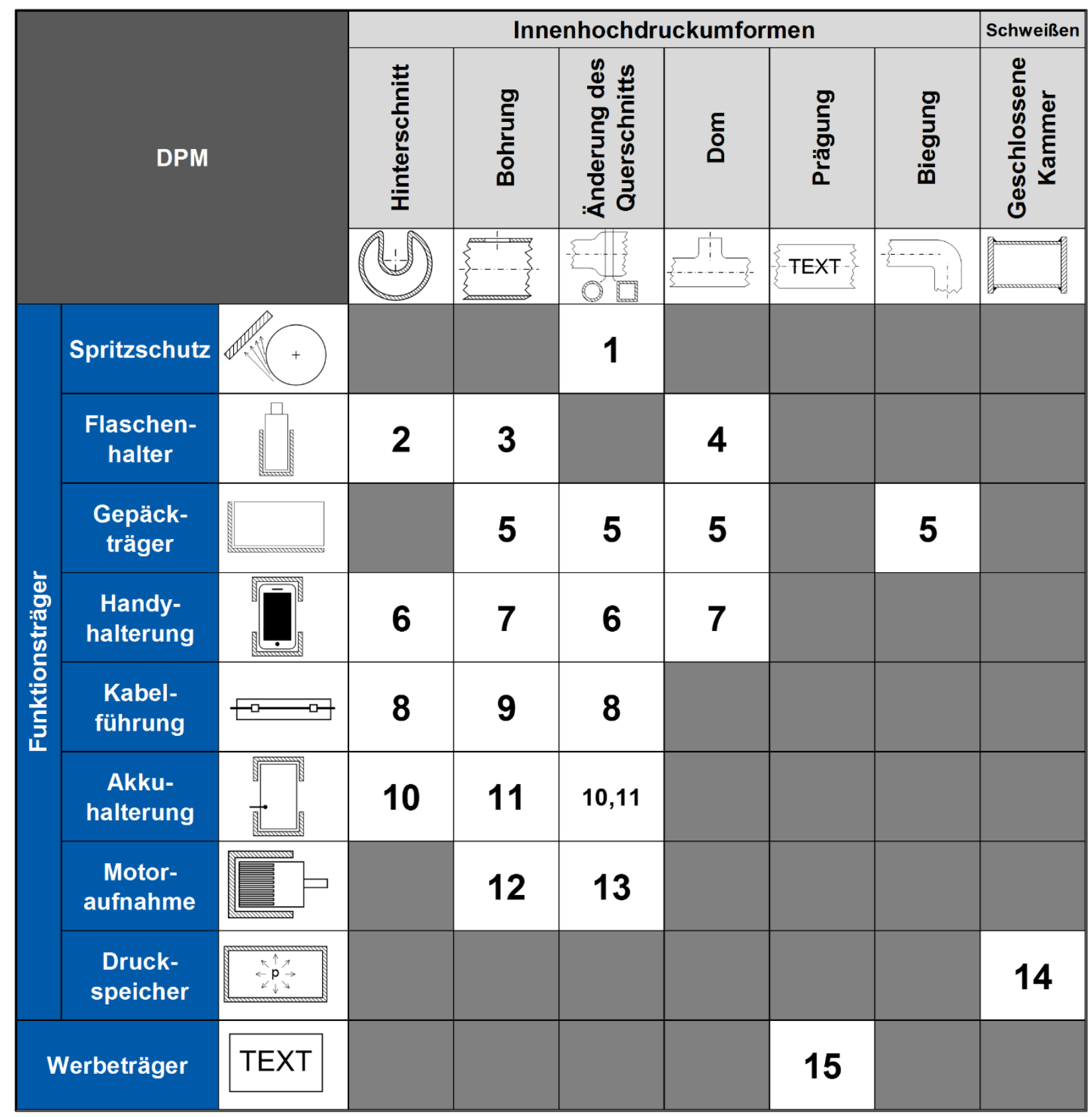




\section{A2 - Design Patterns}

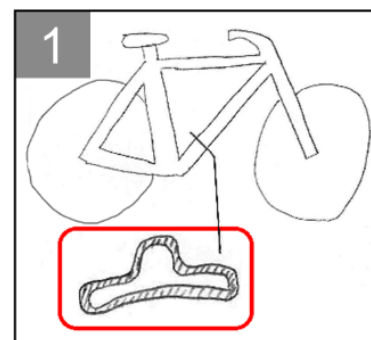

Rahmenintegriertes Schutzblech

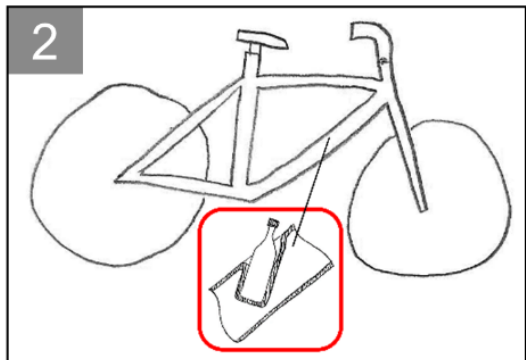

Eingelassener Flaschenhalter

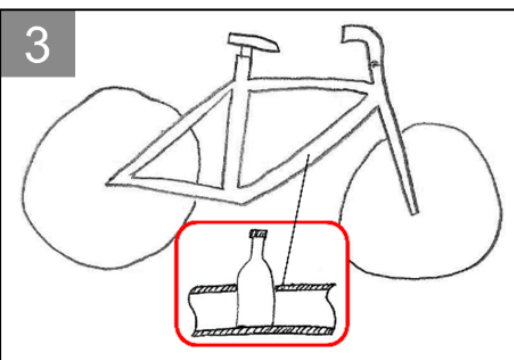

Flaschenhalter-Bohrung

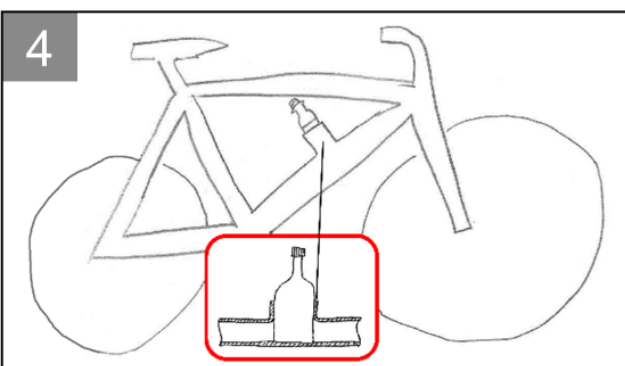

Flaschenhalter-Dom

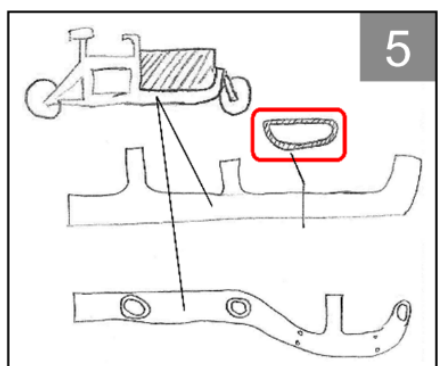

Lastenträger

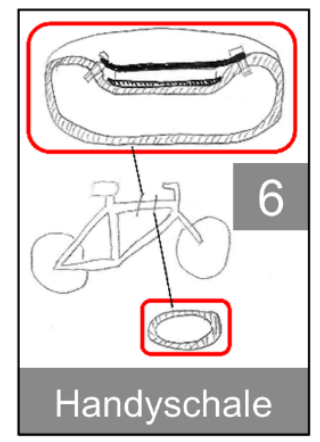

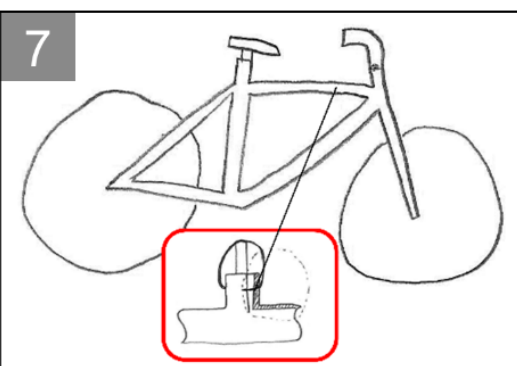

Smartphone-Dom

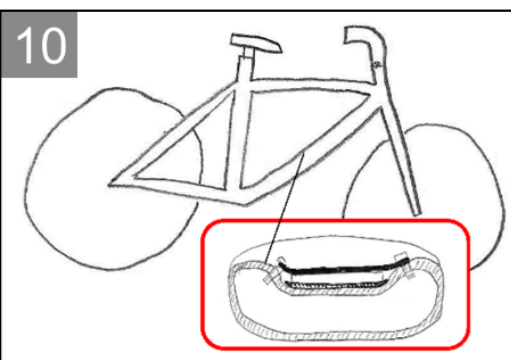

Akkuschale

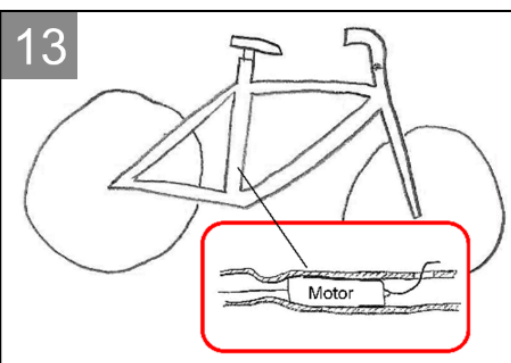

Integrierter Motor

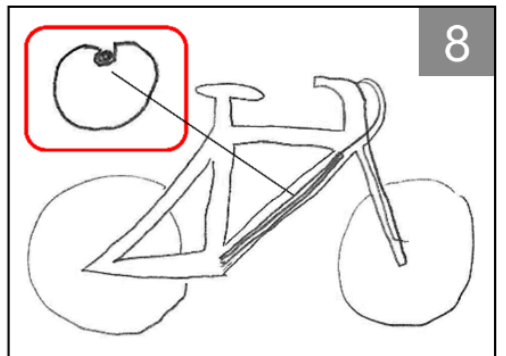

Offener Kabelkanal
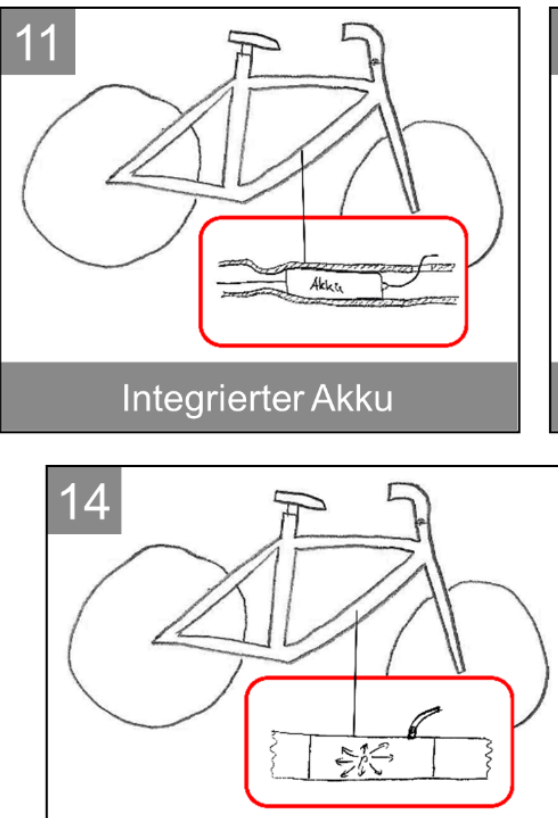

Integrierter Druckspeicher
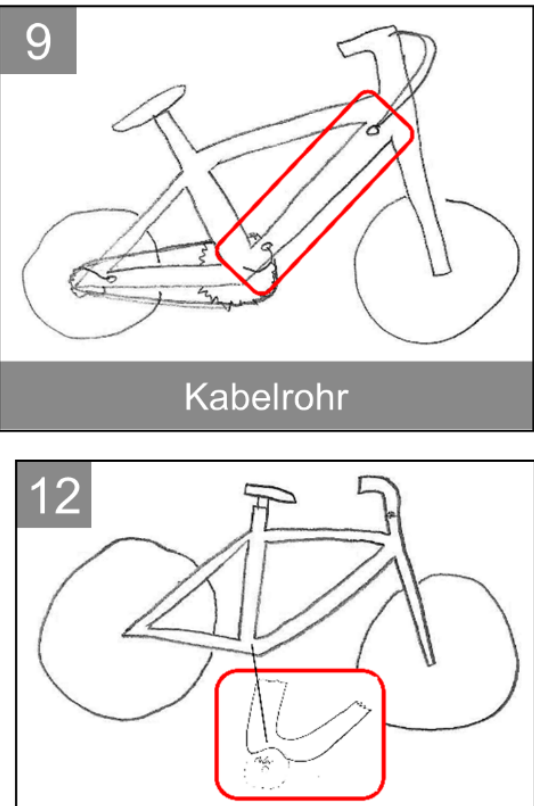

Motorflansch

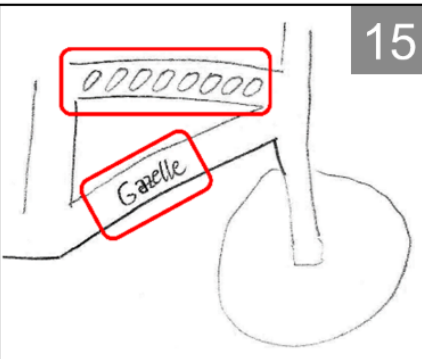

Geprägtes Logo 


\section{A3 - Eingangsdaten für die FE-Simulation der Flaschenhalter}

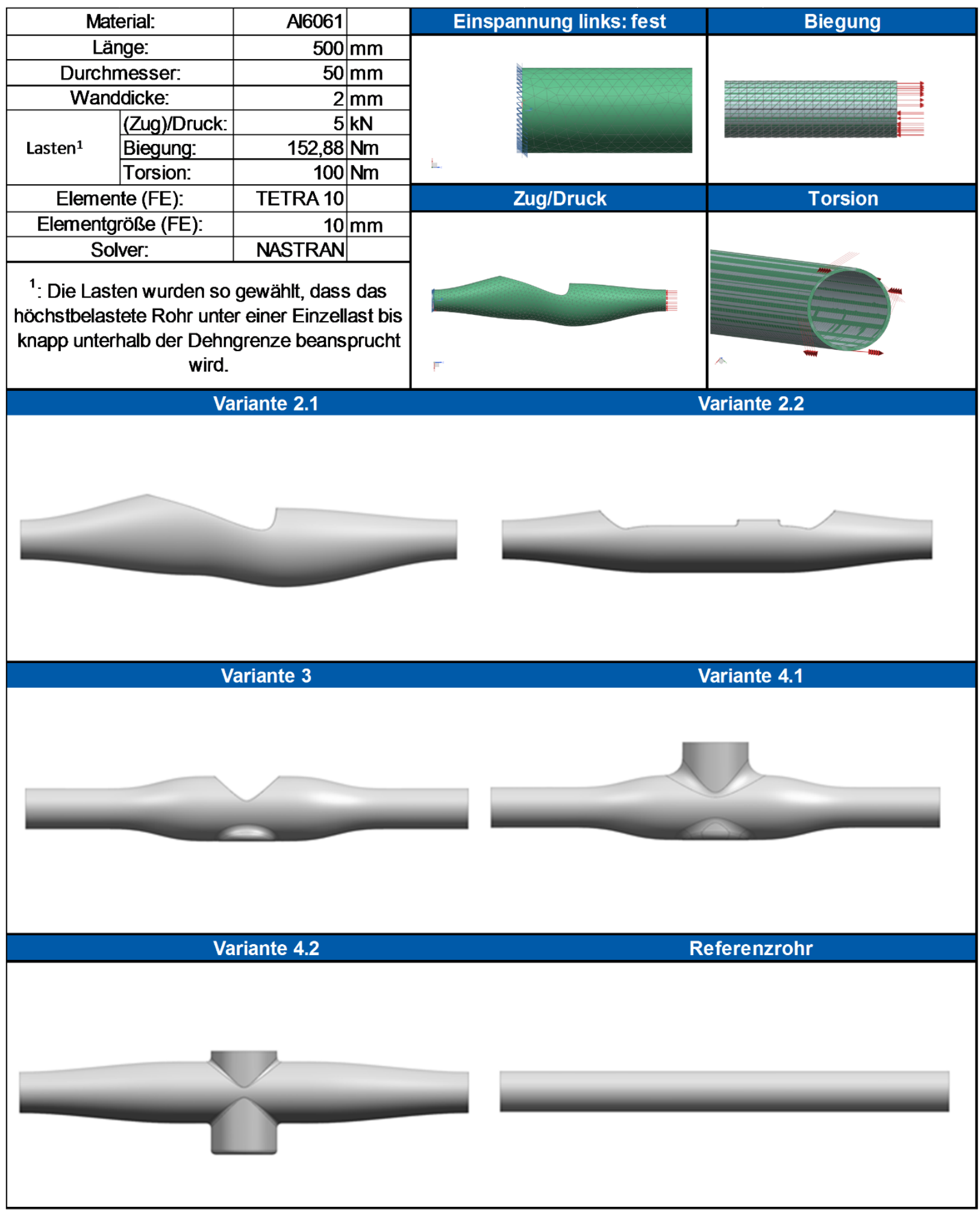




\section{A4 - Spannungen und Steifigkeiten der Flaschenhalter-Varianten}

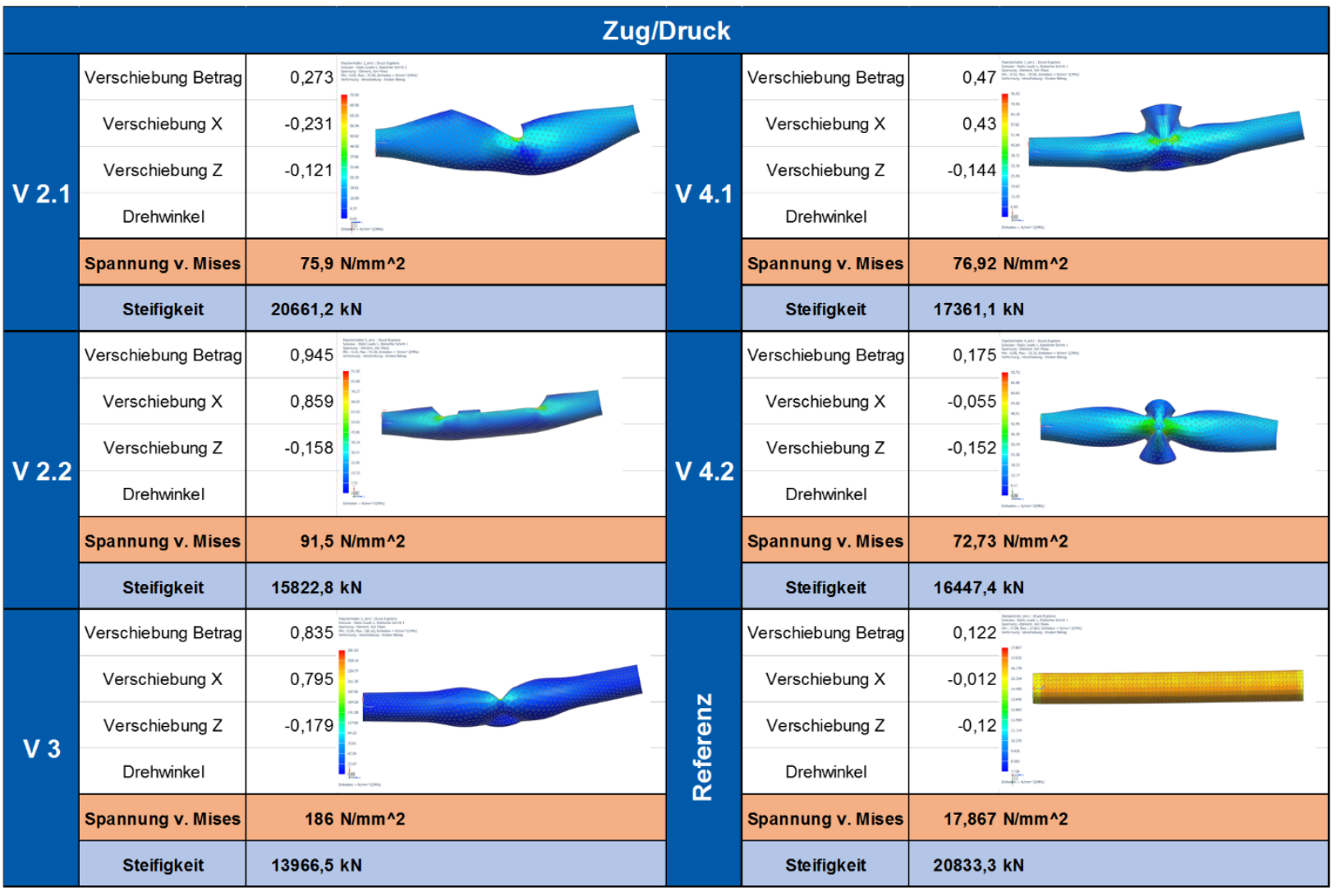

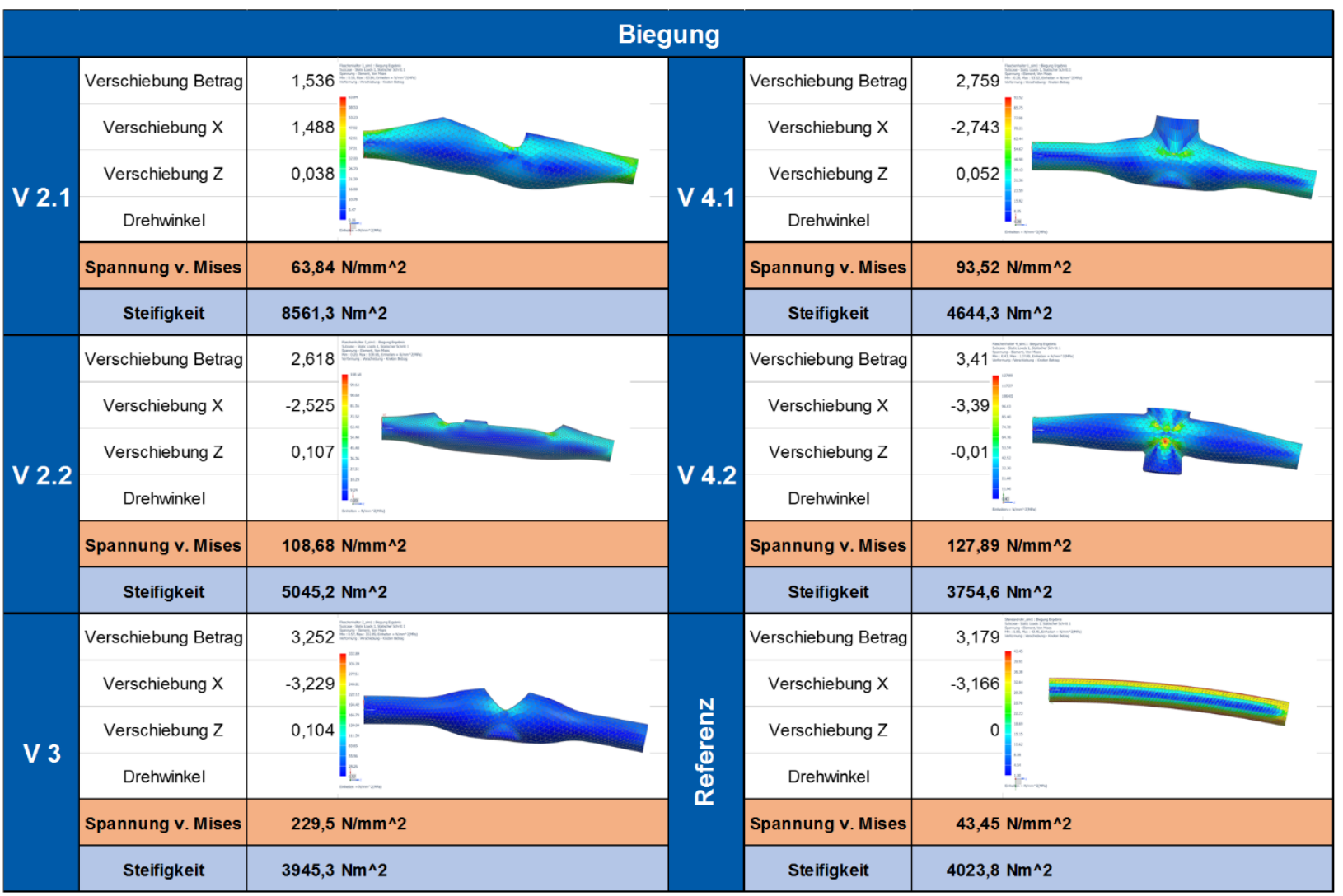




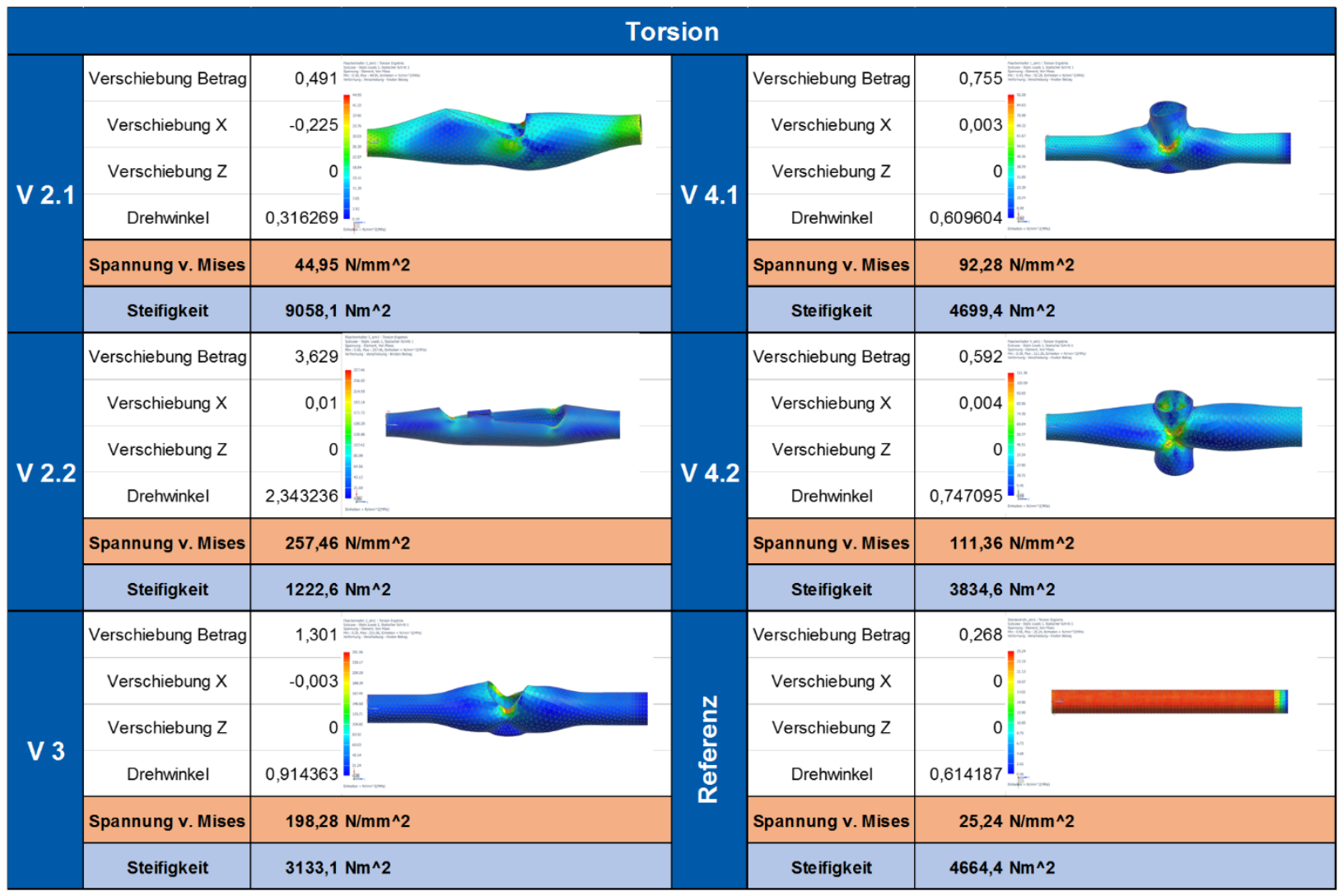

\section{A5 - Paarvergleich der Bewertungskriterien}

\begin{tabular}{|l|c|c|c|c|c|c|}
\cline { 2 - 7 } & Befestigung & Erreichbarkeit & Steifigkeit & $\begin{array}{c}\text { Werkstoff- } \\
\text { ausnutzung }\end{array}$ & Masse & Reinigbarkeit \\
\hline Befestigung & 1 & 2 & 1 & 0 & 0 & 0 \\
\hline Erreichbarkeit & 0 & 1 & 2 & 1 & 2 & 0 \\
\hline Steifigkeit & 1 & 0 & 1 & 0 & 0 & 0 \\
\hline Werkstoffausnutzung & 2 & 1 & 2 & 1 & 0 & 0 \\
\hline Masse & 2 & 0 & 2 & 2 & 1 & 0 \\
\hline Reinigbarkeit & 2 & 2 & 2 & 2 & 2 & 1 \\
\hline \hline Summe & 8 & 6 & 10 & 6 & 5 & 1 \\
\hline Gewichtungsfaktor & $\mathbf{0 , 8}$ & $\mathbf{0 , 6}$ & $\mathbf{1}$ & $\mathbf{0 , 6}$ & $\mathbf{0 , 5}$ & $\mathbf{0 , 1}$ \\
\hline
\end{tabular}




\section{A6 - Eigenschaften der Flaschenhalter-Varianten}

\begin{tabular}{|c|c|c|c|c|c|c|c|c|}
\hline \multicolumn{2}{|c|}{ Kriterien } & Beschreibung & V 2.1 & V 2.2 & V 3 & V 4.1 & V 4.2 & Referenz \\
\hline \multicolumn{2}{|l|}{ Befestigung } & $\begin{array}{l}\text { Länge der Einspannung, um } \\
\text { Biegemomente aufzunehmen }\end{array}$ & $44,30 \mathrm{~mm}$ & $50,00 \mathrm{~mm}$ & $53,20 \mathrm{~mm}$ & $118,00 \mathrm{~mm}$ & $123,00 \mathrm{~mm}$ & - \\
\hline \multicolumn{2}{|c|}{ Erreichbarkeit } & $\begin{array}{l}\text { Länge des Entnahmeweges bis } \\
\text { die Flasche komplett frei ist }\end{array}$ & $53,00 \mathrm{~mm}$ & $50,00 \mathrm{~mm}$ & $78,00 \mathrm{~mm}$ & $118,00 \mathrm{~mm}$ & $123,00 \mathrm{~mm}$ & - \\
\hline \multirow{3}{*}{ Steifigkeit } & \multirow{3}{*}{\begin{tabular}{|l|} 
Zug/Druck \\
Biegung \\
Torsion \\
\end{tabular}} & \multirow{3}{*}{$\begin{array}{l}\text { Die Werte der Variante mit der } \\
\text { jeweils höchsten Steifigkeit } \\
\text { wurden auf } 1 \text { normiert }\end{array}$} & 0,9917 & 0,7595 & 0,6704 & 0,8333 & 0,7895 & 1,0000 \\
\hline & & & 1,0000 & 0,5893 & 0,4608 & 0,5425 & 0,4385 & 0,4700 \\
\hline & & & 1,0000 & 0,1350 & 0,3459 & 0,5188 & 0,4233 & 0,5149 \\
\hline \multirow{3}{*}{$\begin{array}{l}\text { Werkstoff- } \\
\text { ausnutzung }\end{array}$} & \multirow{3}{*}{\begin{tabular}{|l|} 
Zug/Druck \\
Biegung \\
Torsion \\
\end{tabular}} & \multirow{3}{*}{$\begin{array}{l}\text { Anteil der Knoten, deren } \\
\text { Spannung oberhalb von } 50 \% \text { der } \\
\text { maximalen Spannung liegt }\end{array}$} & $0,16 \%$ & $0,80 \%$ & $0,21 \%$ & $0,61 \%$ & $1,30 \%$ & $99,91 \%$ \\
\hline & & & $5,70 \%$ & $0,65 \%$ & $0,20 \%$ & $0,50 \%$ & $2,22 \%$ & $65,75 \%$ \\
\hline & & & $8,43 \%$ & $0,47 \%$ & $0,67 \%$ & $0,57 \%$ & $0,90 \%$ & $96,05 \%$ \\
\hline \multicolumn{2}{|l|}{ Masse } & \begin{tabular}{|l|} 
Gewicht des Rohrabschnittes im \\
Vergleich zum Referenzrohr \\
\end{tabular} & $2116,34 \mathrm{~g}$ & $2081,44 \mathrm{~g}$ & $1424,01 \mathrm{~g}$ & $1599,65 \mathrm{~g}$ & $1739,85 \mathrm{~g}$ & $1180,83 \mathrm{~g}$ \\
\hline \multicolumn{2}{|c|}{ Reinigbarkeit } & $\begin{array}{l}\text { Bewertung der Reinigbarkeit } \\
\text { anhand der Kriterien offen / nicht } \\
\text { offen und mit / ohne Hinterschnitt }\end{array}$ & 3 & 3 & 2 & 2 & 2 & 4 \\
\hline
\end{tabular}

\section{A7 - Spezielle Werteskala}

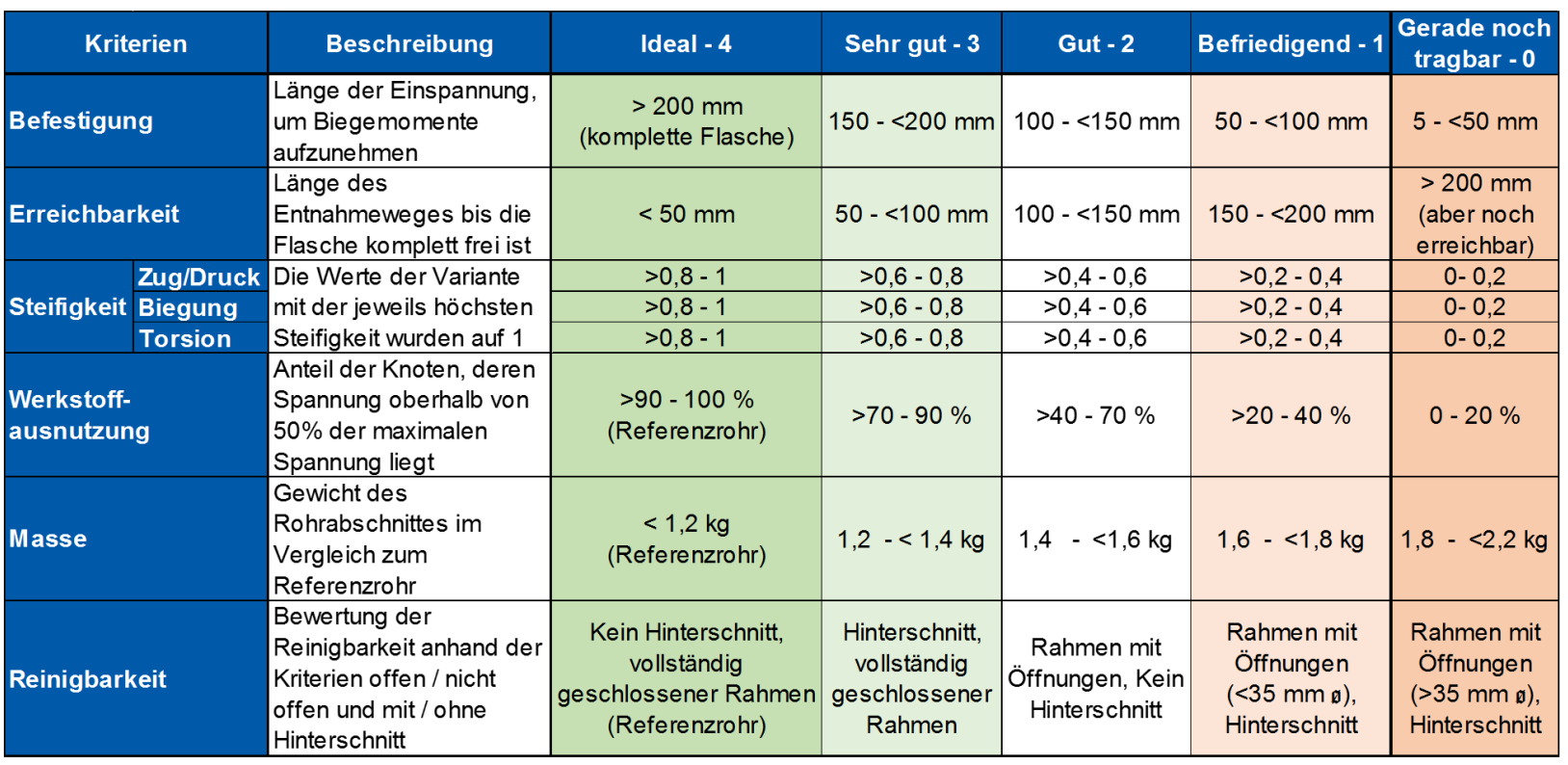




\section{Anhang B - Beispiele für die Anwendung des VP-Modells}

\section{B1 - Verfahren zum Urformen}

Spritzgießen 1 (in Auszügen aus Jaroschek (2013), S. 47):

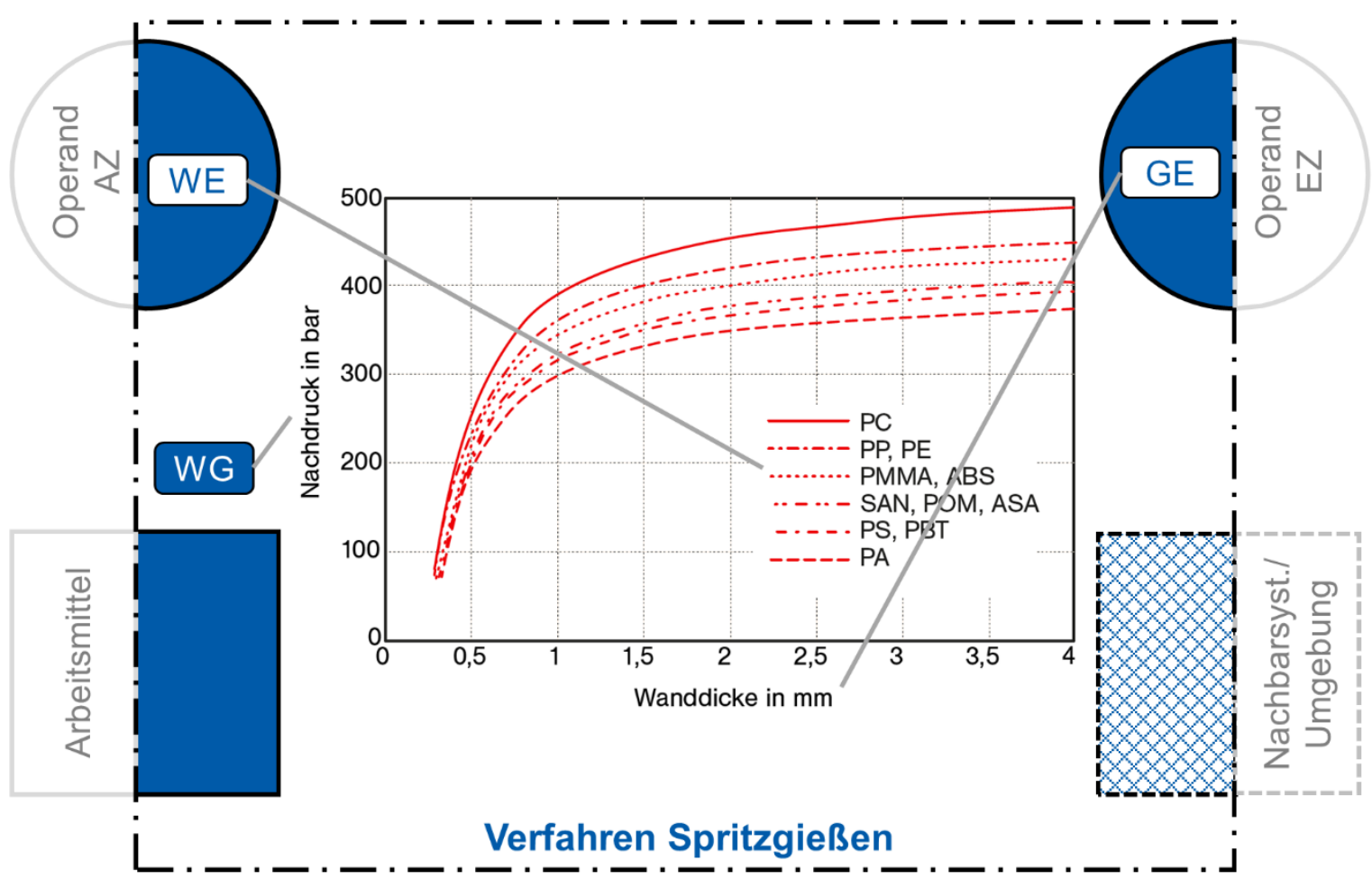

Spritzgießen 2 (in Auszügen aus Johannaber, Michaeli (2004), S. 324):

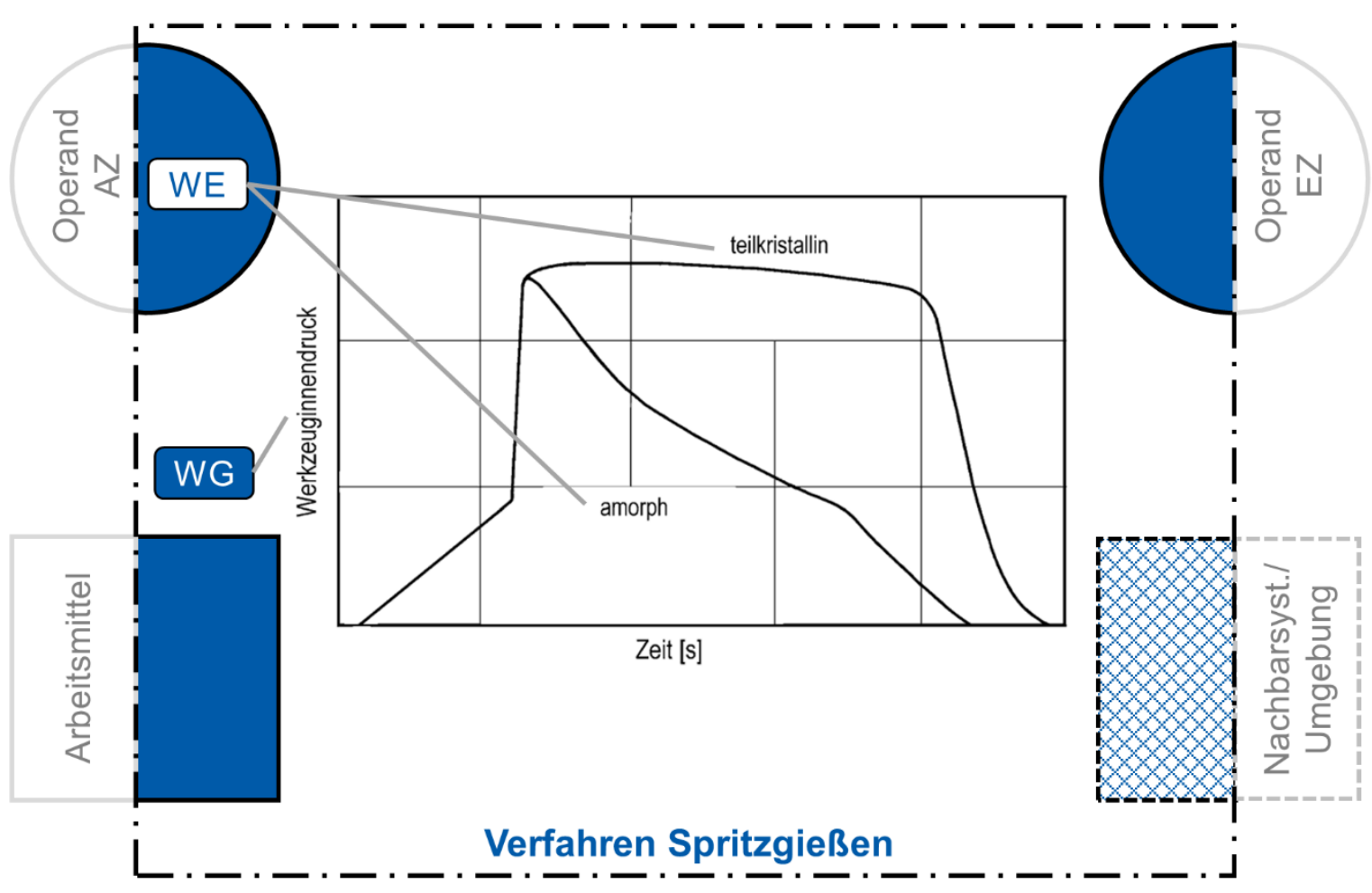




\section{B2 - Verfahren zum Umformen}

Innenhochdruckumformen (in Auszügen aus Noack, Sterzing (2007), S. 15):

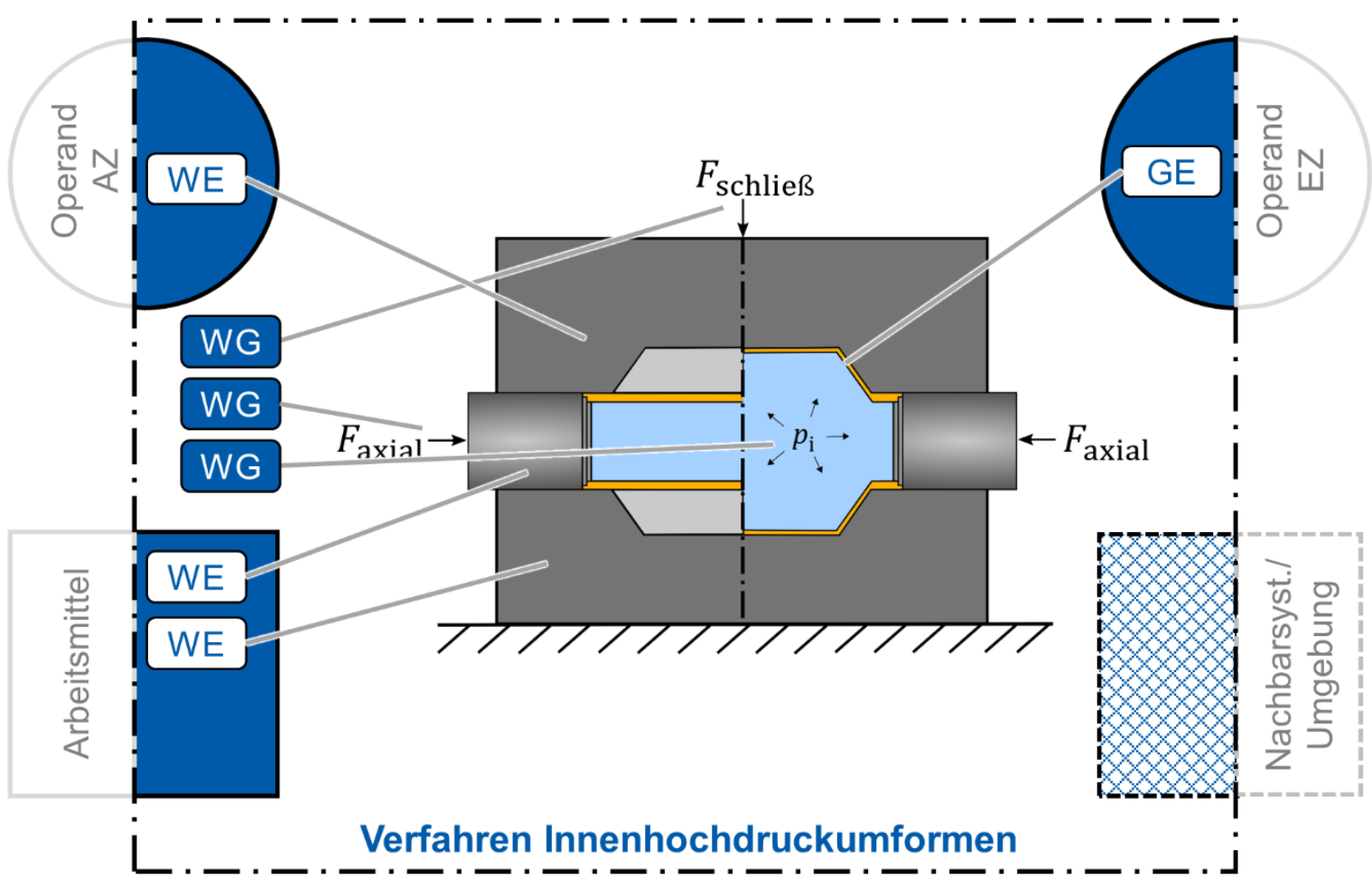

Spaltprofilieren (in Auszügen aus Groche et al. (2007), S. 254):

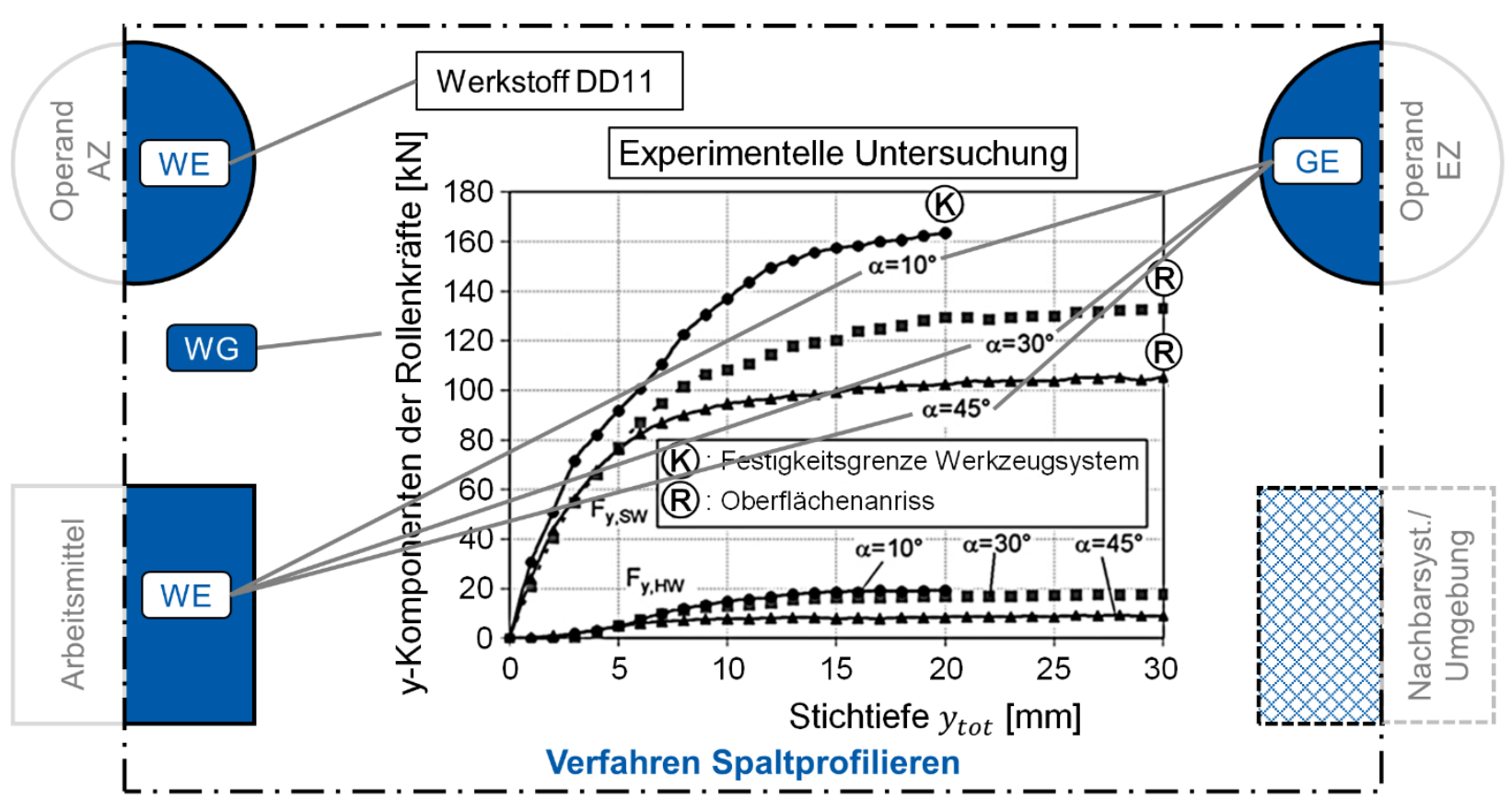




\section{B3 - Verfahren zum Trennen}

Bohren (in Auszügen aus Denkena, Tönshoff (2011), S. 11):

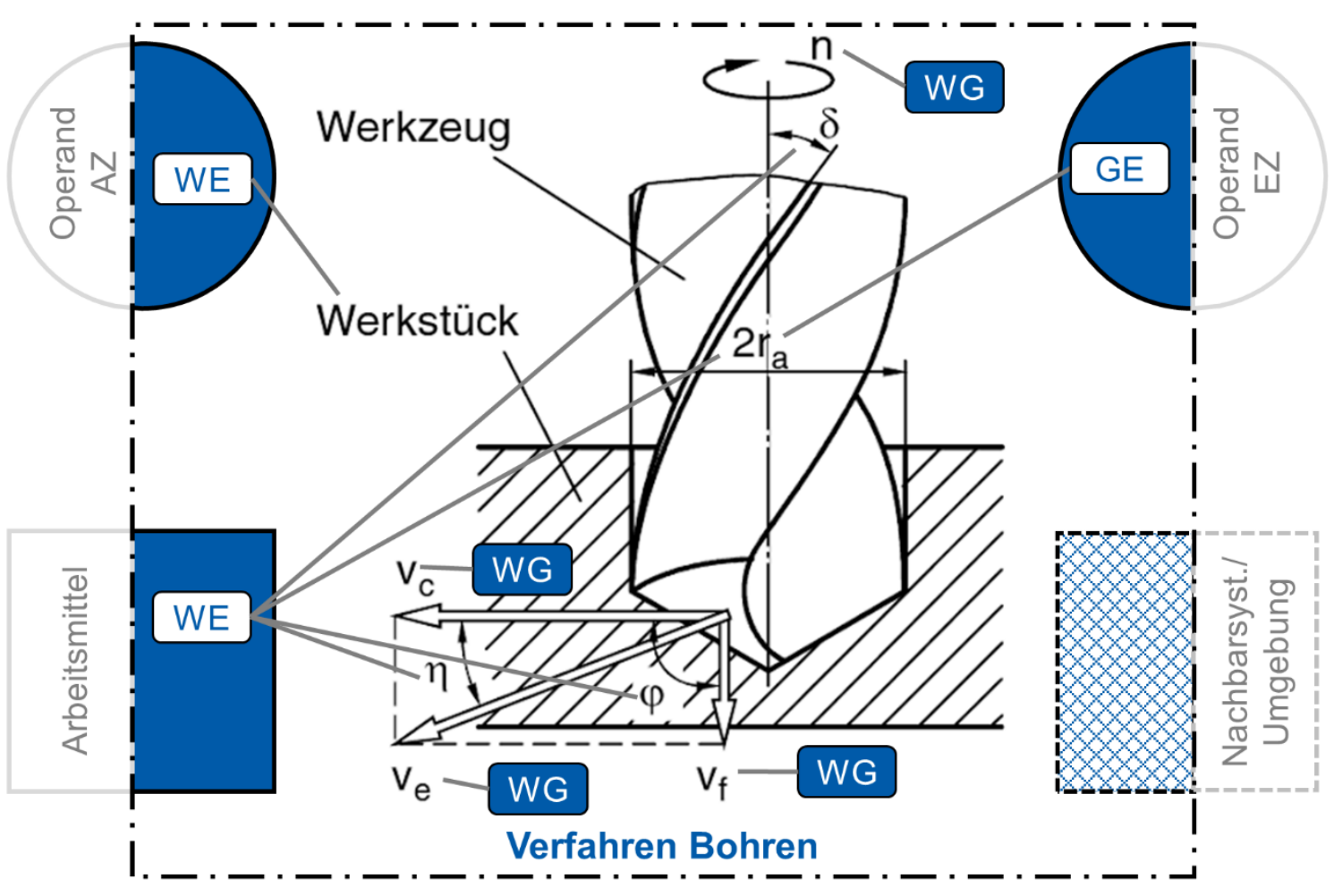

Bohren (Formel in Anlehnung an Denkena, Tönshoff (2011), S. 10):

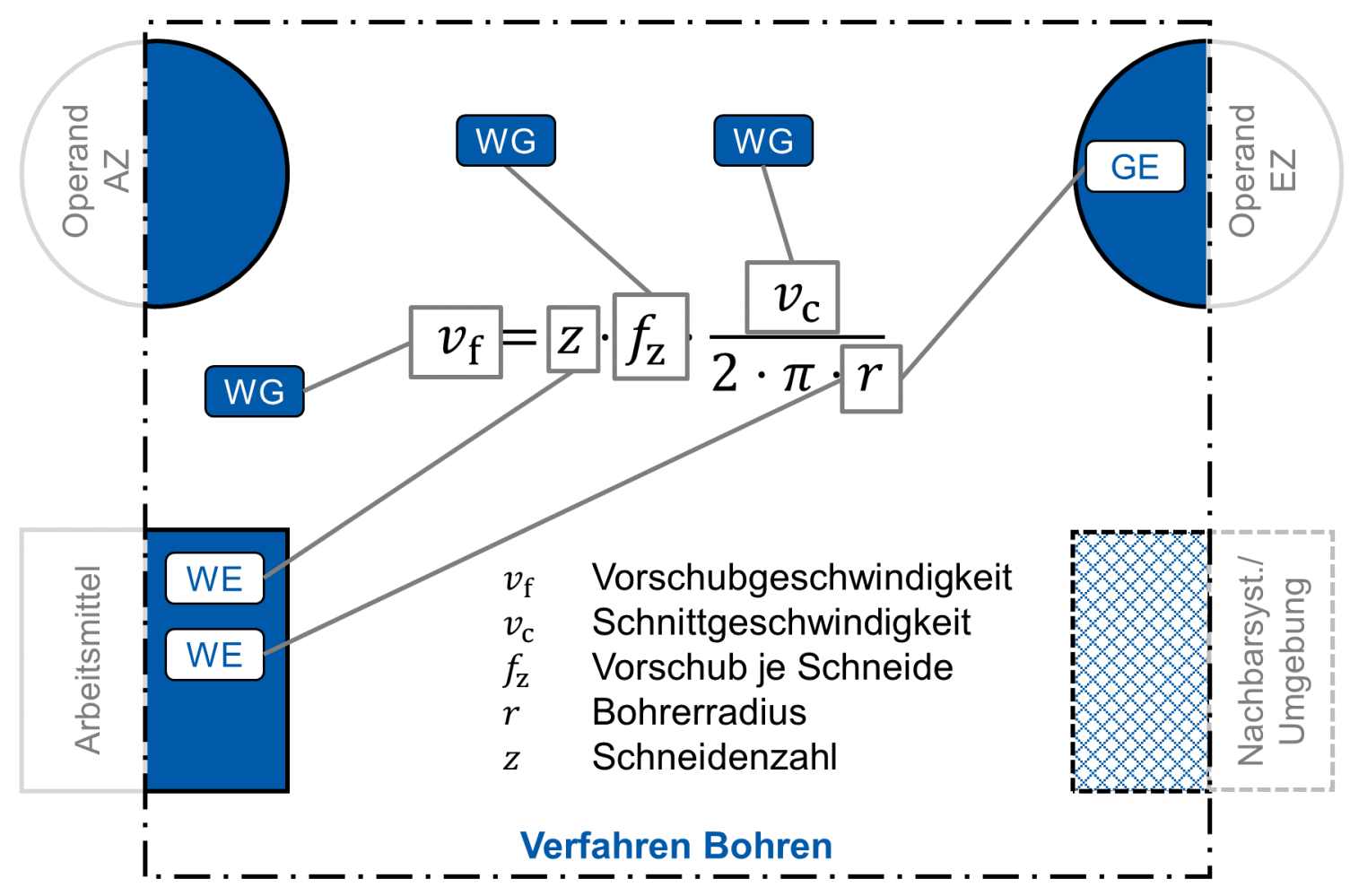




\section{B4 - Verfahren zum Fügen}

Schnappen (Formeln in Anlehnung an Bauer, Althof (1991), S. 307 ff.):

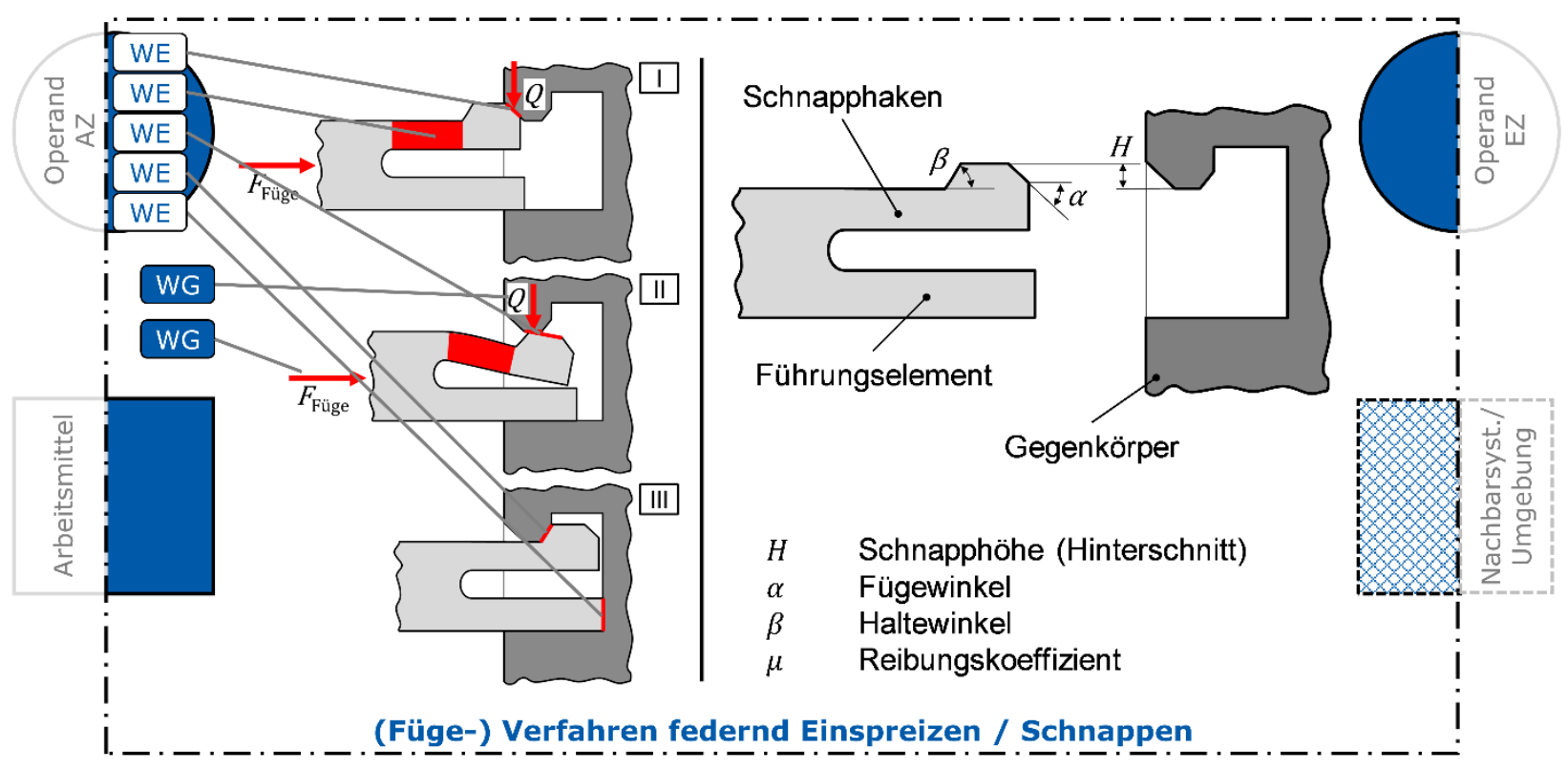

Lichtbogenschweißen (in Auszügen aus Dilthey (1994), S. 10):

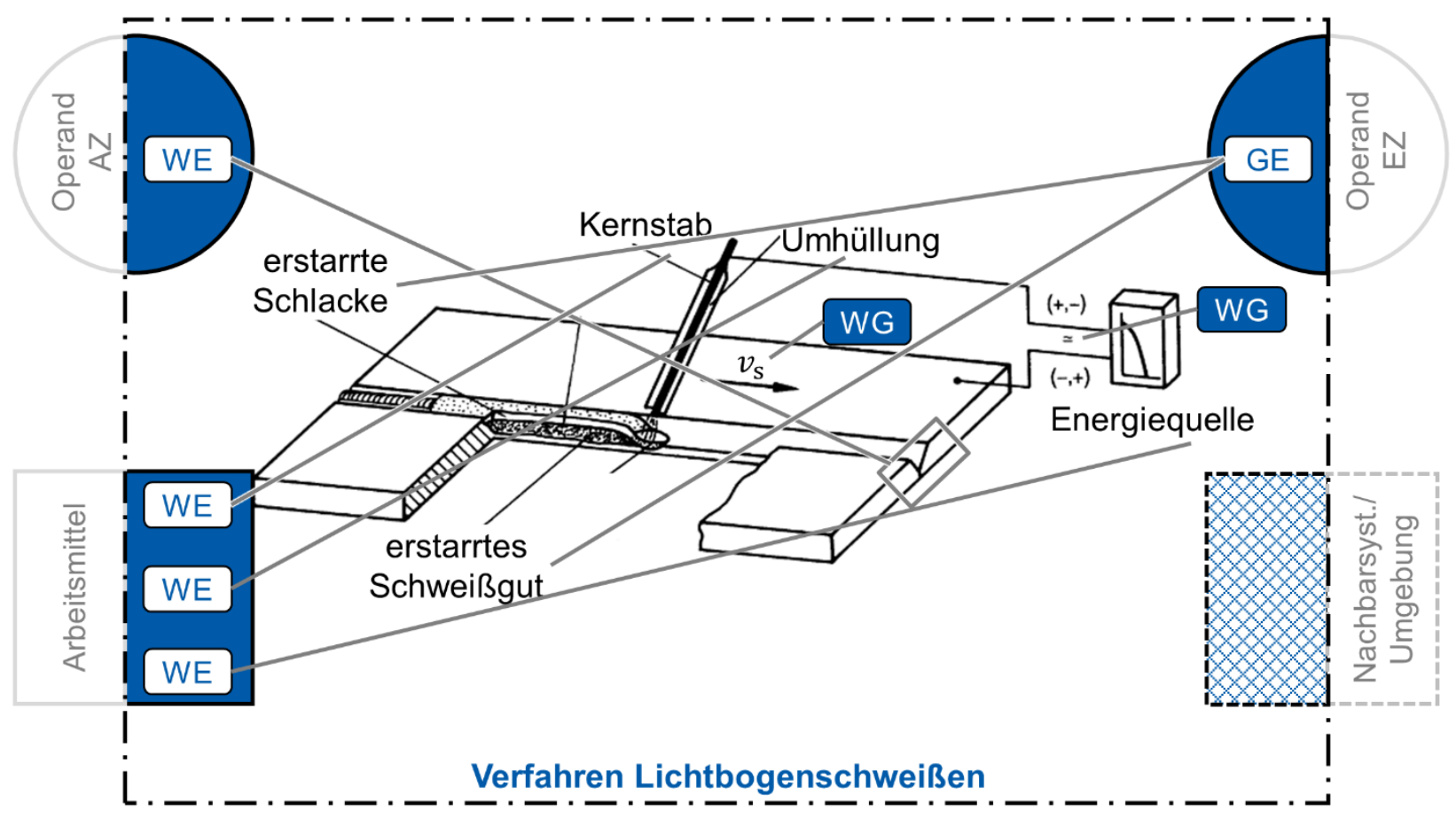




\section{B5 - Verfahren zum Beschichten}

Tampongalvanisieren (in Auszügen aus Hofmann, Spindler (2014), S. 93):

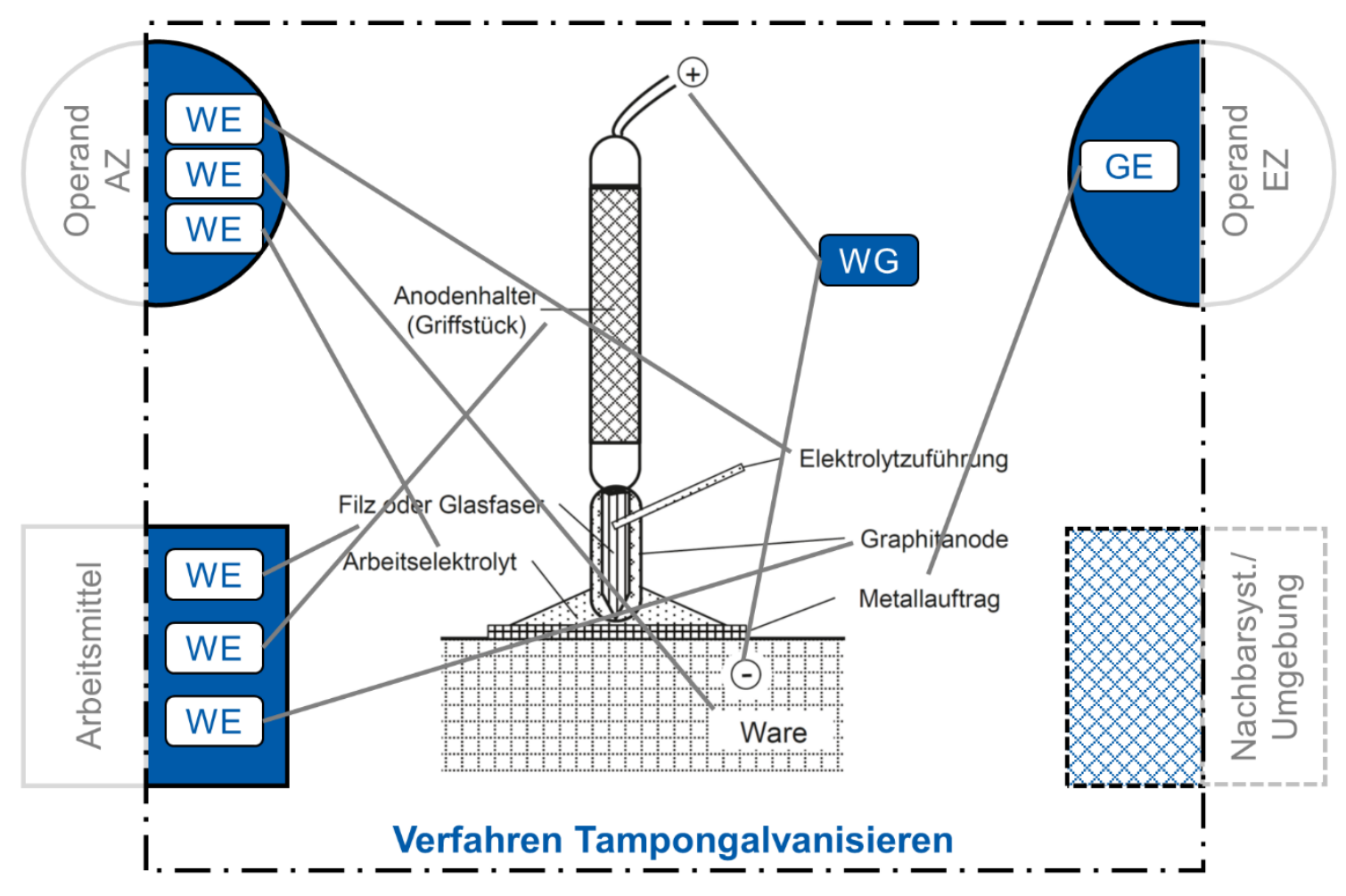

Lackieren mit pneumatischem Sprühstrahl (in Auszügen aus Lake (2016), S. 139):

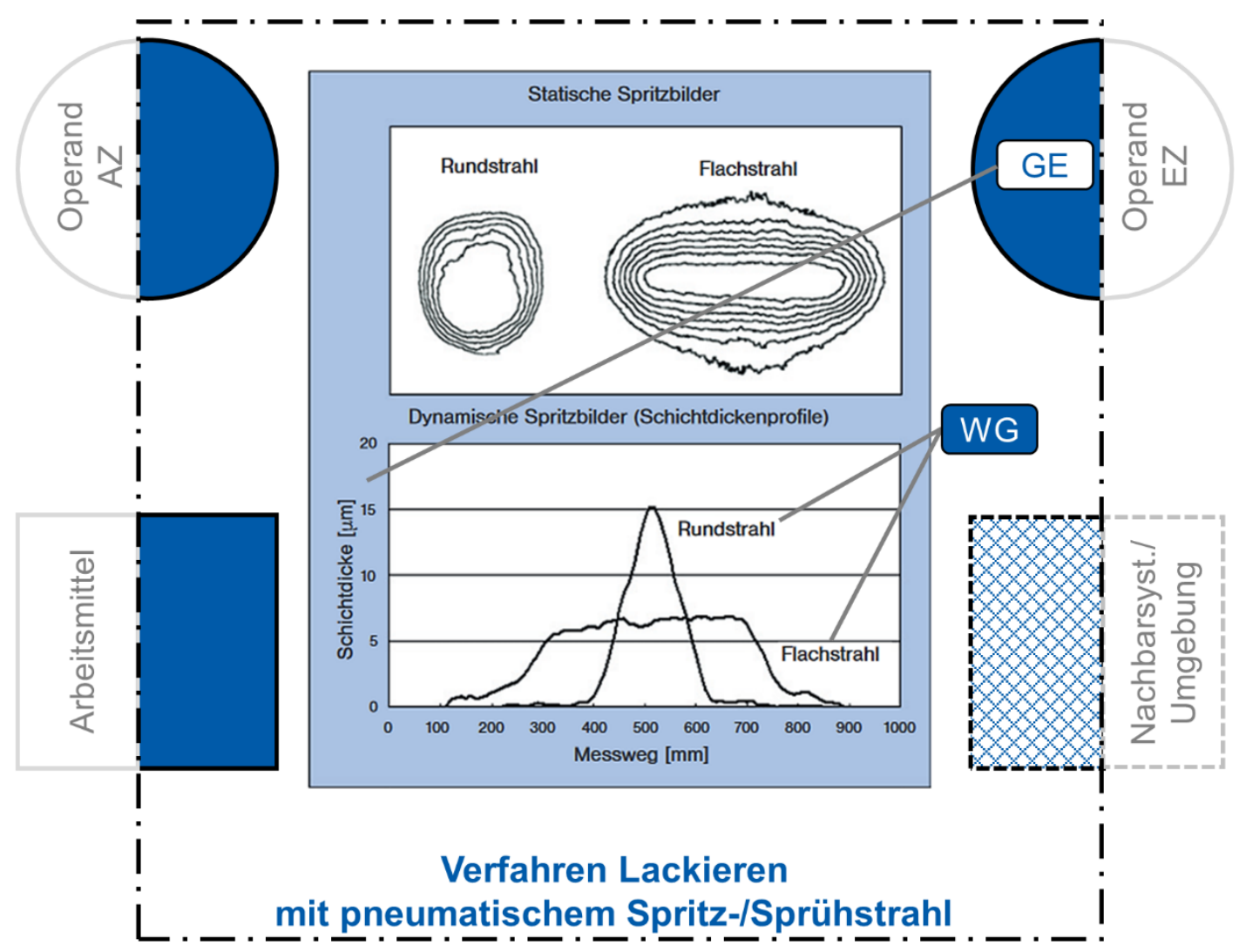




\section{B6 - Verfahren zum Ändern von Stoffeigenschaften}

Induktionshärten (in Anlehnung an Seidel, Hahn (2010), S. 146):

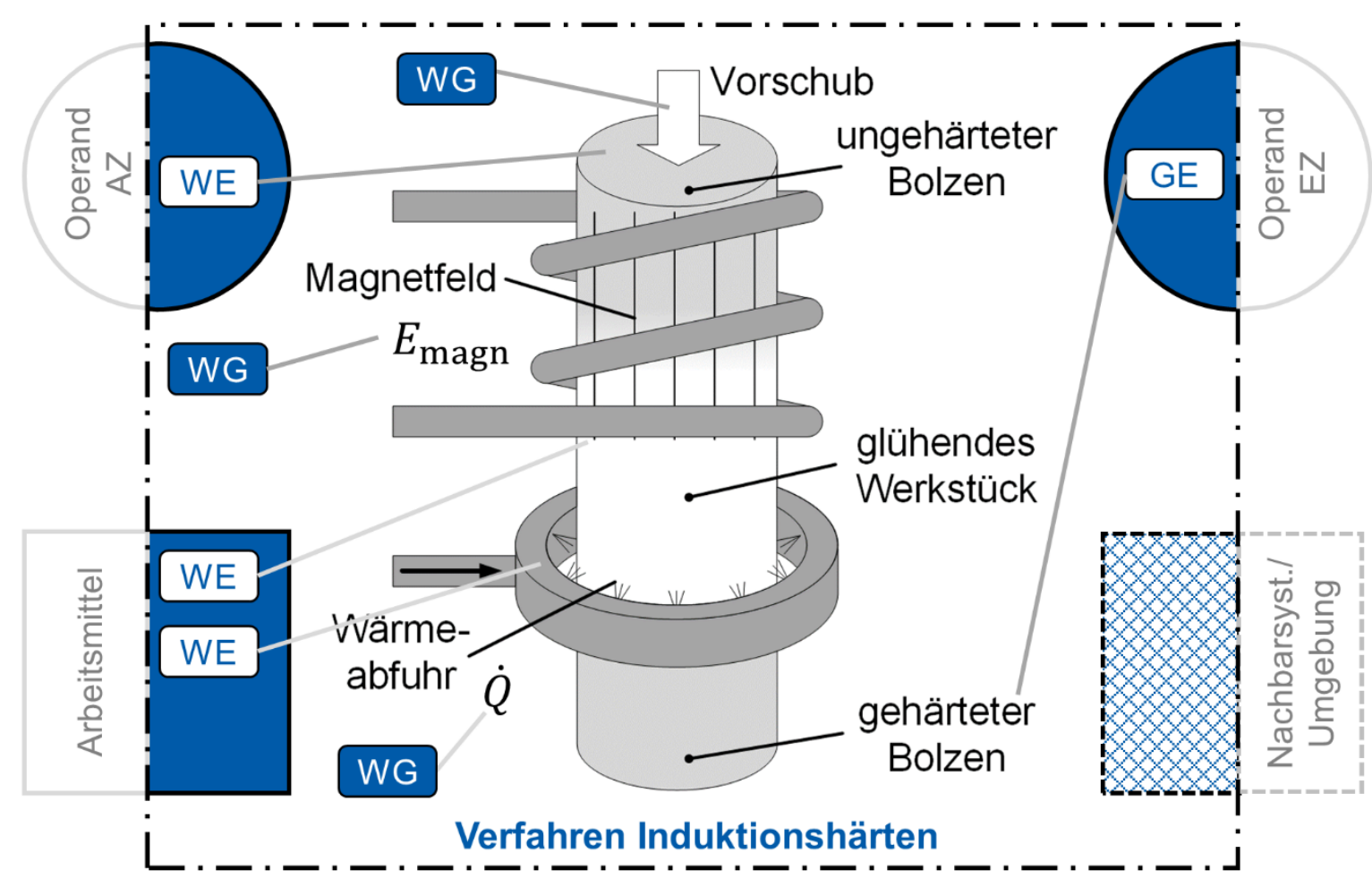

Flammhärten (in Anlehnung an Seidel, Hahn (2010), S. 145):

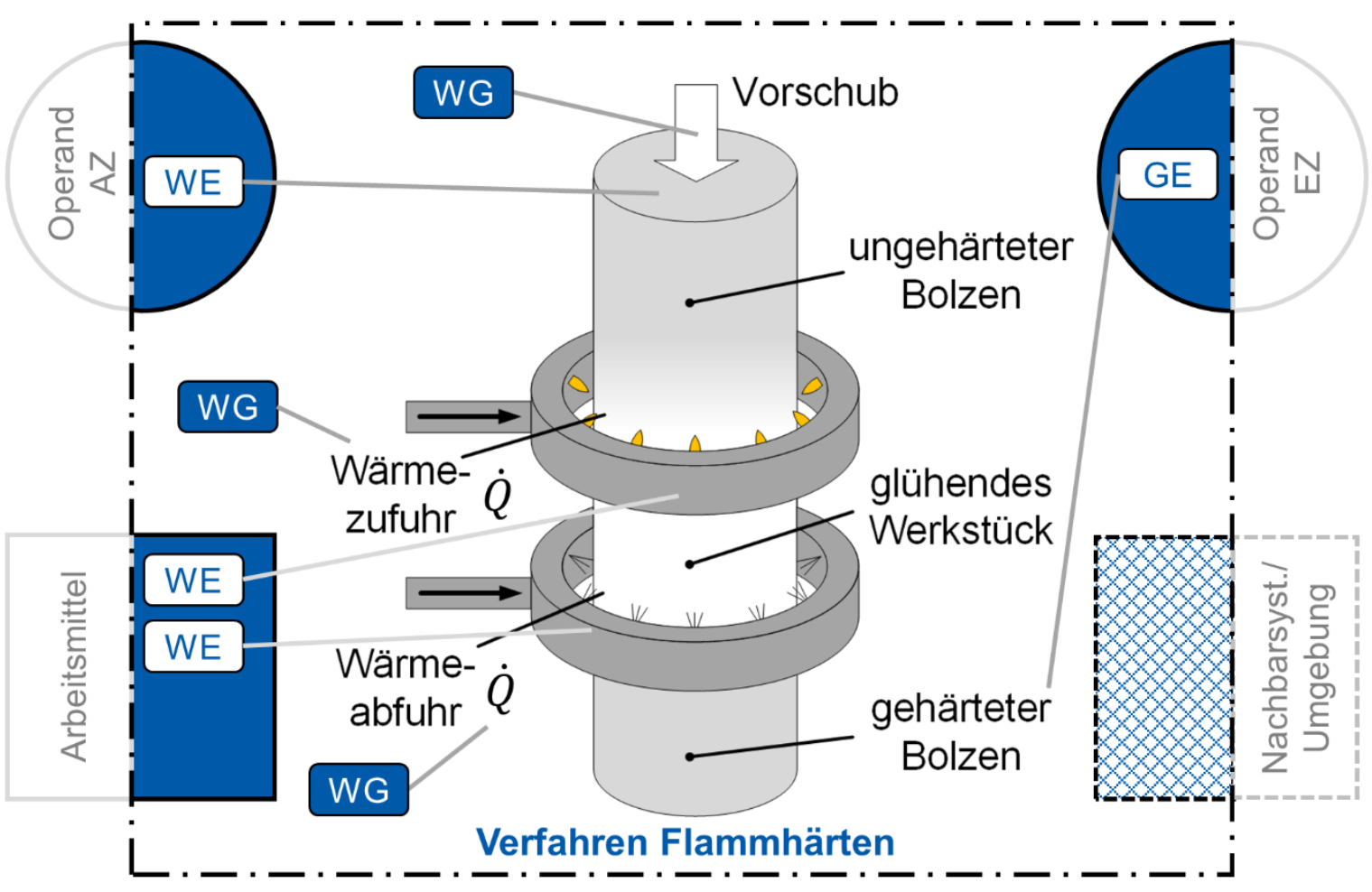




\section{Anhang C - Process Integrated Design Guidelines}

\begin{tabular}{|c|c|c|c|}
\hline Bezeichnung & $\begin{array}{l}\text { Konstruktions- } \\
\text { empfehlung }\end{array}$ & Konsequenz & Erklärung \\
\hline $\begin{array}{l}\text { PIDG } \\
\text { Linearführung }\end{array}$ & $\begin{array}{l}\text { Nutze spaltprofi- } \\
\text { lierte und spaltge- } \\
\text { bogene Flansche } \\
\text { mit ihrer erhöhten } \\
\text { Wälzfestigkeit, um } \\
\text { Wälzkontaktflächen } \\
\text { in Linearführungen } \\
\text { zu realisieren. }\end{array}$ & $\begin{array}{l}\text { erhöhte Le- } \\
\text { bensdauer } \\
\text { der Wälzkon- } \\
\text { takte sowie } \\
\text { erhöhte Ober- } \\
\text { flächenquali- } \\
\text { tät }\end{array}$ & $\begin{array}{l}\text { Das UFG Gefüge } \\
\text { zeichnet sich durch } \\
\text { eine hohe Härte und } \\
\text { geringe Oberflächen- } \\
\text { rauheit aus in Abhän- } \\
\text { gigkeit des Halbzeug- } \\
\text { werkstoffs und be- } \\
\text { stimmter Prozesspara- } \\
\text { meter. (Vgl. Ahmels et } \\
\text { al. (2017).) }\end{array}$ \\
\hline
\end{tabular}

\begin{tabular}{|c|c|c|c|}
\hline $\begin{array}{l}\text { PIDG } \\
\text { Kraftüber- } \\
\text { tragung }\end{array}$ & $\begin{array}{l}\text { Nutze spaltprofi- } \\
\text { lierte und spaltge- } \\
\text { bogene Flansche } \\
\text { mit ihrer erhöhten } \\
\text { Festigkeit in hoch } \\
\text { beanspruchten Be- } \\
\text { reichen innerhalb } \\
\text { profilartiger Bauteile, } \\
\text { die der Kraftübertra- } \\
\text { gung dienen. }\end{array}$ & $\begin{array}{l}\text { erhöhte Be- } \\
\text { anspruchbar- } \\
\text { keit }\end{array}$ & $\begin{array}{l}\text { Die UFG Gefügestruk- } \\
\text { tur weist eine erhöhte } \\
\text { Festigkeit auf in Abhän- } \\
\text { gigkeit des Halbzeug- } \\
\text { werkstoffs und be- } \\
\text { stimmter Prozesspara- } \\
\text { meter. (Vgl. Ahmels et } \\
\text { al. (2017).) }\end{array}$ \\
\hline $\begin{array}{l}\text { PIDG } \\
\text { Kerbwirkung }\end{array}$ & $\begin{array}{l}\text { Nutze spaltprofi- } \\
\text { lierte und spaltge- } \\
\text { bogene Flansche } \\
\text { mit ihrer geringeren } \\
\text { Kerbempfindlichkeit, } \\
\text { um Funktionsele- } \\
\text { mente in profilartige } \\
\text { Bauteile zu integrie- } \\
\text { ren. }\end{array}$ & $\begin{array}{l}\text { erhöhte Be- } \\
\text { lastbarkeit } \\
\text { des Formele- } \\
\text { ments }\end{array}$ & $\begin{array}{l}\text { Der Vergleich verschie- } \\
\text { dener Wöhler-Kurven } \\
\text { deutet auf eine gerin- } \\
\text { gere Kerbempfindlich- } \\
\text { keit des UFG Gefüges } \\
\text { hin. (Vgl. Ahmels et al. } \\
(2017) .)\end{array}$ \\
\hline $\begin{array}{l}\text { PIDG } \\
\text { Haltevorrich- } \\
\text { tung } 1\end{array}$ & $\begin{array}{l}\text { Nutze innenhoch- } \\
\text { druckumgeformte } \\
\text { Dome, um rotati- } \\
\text { onssymmetrische } \\
\text { Bauteile im Rah- } \\
\text { menrohr von Fahrrä- } \\
\text { dern zu fixieren. }\end{array}$ & $\begin{array}{l}\text { Einzelteile, } \\
\text { Fertigungs- } \\
\text { prozesse und } \\
\text { Montagepro- } \\
\text { zesse können } \\
\text { eingespart } \\
\text { werden. }\end{array}$ & $\begin{array}{l}\text { IHU bietet die Möglich- } \\
\text { keit Dome auszufor- } \\
\text { men, wobei deren } \\
\text { Höhe insbesondere } \\
\text { durch das Werkzeug } \\
\text { und das Werkstück be- } \\
\text { einflusst wird. (Vgl. } \\
\text { Bahn et al. (2007).) }\end{array}$ \\
\hline
\end{tabular}




\begin{tabular}{|c|c|c|c|}
\hline Bezeichnung & $\begin{array}{l}\text { Konstruktions- } \\
\text { empfehlung }\end{array}$ & Konsequenz & Erklärung \\
\hline $\begin{array}{l}\text { PIDG } \\
\text { Haltevorrich- } \\
\text { tung } 2\end{array}$ & $\begin{array}{l}\text { Nutze während des } \\
\text { IHU-Prozesses ein- } \\
\text { gebrachte Bohrun- } \\
\text { gen, um rotations- } \\
\text { symmetrische Bau- } \\
\text { teile im Rahmenrohr } \\
\text { von Fahrrädern zu fi- } \\
\text { xieren. }\end{array}$ & $\begin{array}{l}\text { Einzelteile, } \\
\text { Fertigungs- } \\
\text { prozesse und } \\
\text { Montagepro- } \\
\text { zesse können } \\
\text { eingespart } \\
\text { werden. }\end{array}$ & $\begin{array}{l}\text { Durch einen Stempel } \\
\text { im IHU-Werkzeug kön- } \\
\text { nen in das unter einem } \\
\text { Innendruck stehende } \\
\text { Rohr prozessintegriert } \\
\text { Löcher gestanzt wer- } \\
\text { den. (Vgl. Bahn et al. } \\
(2007) \text {.) }\end{array}$ \\
\hline $\begin{array}{l}\text { PIDG } \\
\text { Haltevorrich- } \\
\text { tung } 3\end{array}$ & $\begin{array}{l}\text { Nutze die über der } \\
\text { Rohrlänge variab- } \\
\text { len Querschnittgeo- } \\
\text { metrien innenhoch- } \\
\text { druckumgeformter } \\
\text { Rohre, um Hinter- } \\
\text { schnitte zu realisie- } \\
\text { ren, in denen zusätz- } \\
\text { liche Bauteile fixiert } \\
\text { werden können. }\end{array}$ & $\begin{array}{l}\text { Einzelteile, } \\
\text { Fertigungs- } \\
\text { prozesse und } \\
\text { Montagepro- } \\
\text { zesse können } \\
\text { eingespart } \\
\text { werden. }\end{array}$ & $\begin{array}{l}\text { Die Größe des Hinter- } \\
\text { schnitts und dessen } \\
\text { Radien werden durch } \\
\text { den Werkstoff des } \\
\text { Halbzeugs, dessen } \\
\text { Wanddicke sowie die } \\
\text { realisierbaren Umform- } \\
\text { kräfte limitiert. (Vgl. } \\
\text { Bahn et al. (2007).) }\end{array}$ \\
\hline $\begin{array}{l}\text { PIDG } \\
\text { Lösbare Ver- } \\
\text { bindung }\end{array}$ & $\begin{array}{l}\text { Nutze spaltprofi- } \\
\text { lierte Flansche auf- } \\
\text { grund ihrer hohen } \\
\text { Festigkeit als } \\
\text { Schnapphaken. }\end{array}$ & $\begin{array}{l}\text { Realisierung } \\
\text { höherer Ver- } \\
\text { formungen } \\
\text { der Schnapp- } \\
\text { verbindung } \\
\text { beim Fügen } \\
\text { und Lösen }\end{array}$ & $\begin{array}{l}\text { Bei gleichem E-Modul } \\
\text { ermöglicht die erhöhte } \\
\text { Festigkeit der Flansche } \\
\text { höhere Verformungen. }\end{array}$ \\
\hline $\begin{array}{l}\text { PIDG } \\
\text { Schmier- } \\
\text { stoffförderung }\end{array}$ & $\begin{array}{l}\text { Nutze rotationssym- } \\
\text { metrische Rillen ge- } \\
\text { drehter Wellen als } \\
\text { Förderelement für } \\
\text { Schmierstoffe. }\end{array}$ & $\begin{array}{l}\text { ständige Neu- } \\
\text { benetzung } \\
\text { und gleich- } \\
\text { mäßigere } \\
\text { Schmierstoff- } \\
\text { verteilung }\end{array}$ & $\begin{array}{l}\text { Drehen erzeugt umlau- } \\
\text { fende schraubenför- } \\
\text { mige Textur auf der } \\
\text { Bauteiloberfläche, die } \\
\text { insbesondere von der } \\
\text { Vorschubgeschwindig- } \\
\text { keit beim Drehen ab- } \\
\text { hängt. (Vgl. Schönherr } \\
\text { (2002).) }\end{array}$ \\
\hline
\end{tabular}




\begin{tabular}{|c|c|c|c|}
\hline Bezeichnung & $\begin{array}{l}\text { Konstruktions- } \\
\text { empfehlung }\end{array}$ & Konsequenz & Erklärung \\
\hline $\begin{array}{l}\text { PIDG } \\
\text { Welle unter } \\
\text { Biegung }\end{array}$ & $\begin{array}{l}\text { Nutze die Oberflä- } \\
\text { chenverfestigung ge- } \\
\text { walzter Bauteile, um } \\
\text { die Beanspruchbar- } \\
\text { keit von biegebelas- } \\
\text { teten Wellen zu er- } \\
\text { höhen. }\end{array}$ & $\begin{array}{l}\text { höhere Bie- } \\
\text { gemomente } \\
\text { ertragbar }\end{array}$ & $\begin{array}{l}\text { Kaltverfestigung beim } \\
\text { Walzen erhöht Festig- } \\
\text { keit der Oberfläche } \\
\text { (Vgl. Klocke, König } \\
\text { (2006).); beim Biegen } \\
\text { werden vor allem die } \\
\text { Randbereiche des Bau- } \\
\text { teils hoch beansprucht }\end{array}$ \\
\hline $\begin{array}{l}\text { PIDG } \\
\text { Leitfähigkeit } \\
\text { von Be- } \\
\text { schichtungen }\end{array}$ & $\begin{array}{l}\text { Nutze Beschichtun- } \\
\text { gen mit definierter } \\
\text { Leitfähigkeit, um die } \\
\text { Leitfähigkeit von } \\
\text { stromdurchflosse- } \\
\text { nen Bauteilen zu } \\
\text { verändern. }\end{array}$ & $\begin{array}{l}\text { Erhöhung } \\
\text { oder auch } \\
\text { Verhinderung } \\
\text { eines elektri- } \\
\text { schen Stroms }\end{array}$ & $\begin{array}{l}\text { Unterschiedliche Be- } \\
\text { schichtungsstoffe (z. B. } \\
\text { Metalle oder Polymere) } \\
\text { weisen unterschiedli- } \\
\text { che Leitfähigkeit auf }\end{array}$ \\
\hline $\begin{array}{l}\text { PIDG } \\
\text { Dämpfung }\end{array}$ & $\begin{array}{l}\text { Nutze die Elastizität } \\
\text { von Dickschichtkle- } \\
\text { bungen, um gezielt } \\
\text { Dämpfung im Bau- } \\
\text { teil zu erzeugen. }\end{array}$ & $\begin{array}{l}\text { Schwin- } \\
\text { gungsdämp- } \\
\text { fung }\end{array}$ & $\begin{array}{l}\text { Dickschichtklebungen } \\
\text { erhöhen die Dämpfung } \\
\text { im Bauteil. (Vgl. Pröbs- } \\
\text { ter (2013).) }\end{array}$ \\
\hline $\begin{array}{l}\text { PIDG } \\
\text { Schadens- } \\
\text { detektion }\end{array}$ & $\begin{array}{l}\text { Nutze mehrlagige } \\
\text { Beschichtungen } \\
\text { aus metallischen und } \\
\text { nichtmetallischen } \\
\text { Werkstoffen, um eine } \\
\text { lastabhängige Strom- } \\
\text { leitung im Bauteil zu } \\
\text { erzeugen. }\end{array}$ & $\begin{array}{l}\text { Überlast oder } \\
\text { Schädigung } \\
\text { detektieren }\end{array}$ & $\begin{array}{l}\text { Überlast oder Schlag- } \\
\text { schäden führen zu Zer- } \\
\text { störung von nichtmetal- } \\
\text { lischen Trennschichten, } \\
\text { sodass leitfähige } \\
\text { Schichten verbunden } \\
\text { werden. }\end{array}$ \\
\hline $\begin{array}{l}\text { PIDG } \\
\text { Flüssig- } \\
\text { keitsspeicher }\end{array}$ & $\begin{array}{l}\text { Nutze die Porosität } \\
\text { gesinterter Bau- } \\
\text { teile, um flüssige } \\
\text { Stoffe im Bauteil zu } \\
\text { speichern. }\end{array}$ & $\begin{array}{l}\text { Dämpfung } \\
\text { des Bauteils } \\
\text { gezielt durch } \\
\text { Flüssigkeits- } \\
\text { menge ein- } \\
\text { stellbar }\end{array}$ & $\begin{array}{l}\text { Gesinterte Bauteile } \\
\text { weisen abhängig von } \\
\text { der Wahl der Pro- } \\
\text { zessparameter eine } \\
\text { hohe Porosität auf. } \\
\text { (Vgl. Kieffer, Hotop } \\
\text { (1948).) }\end{array}$ \\
\hline
\end{tabular}




\begin{tabular}{|c|c|c|c|}
\hline Bezeichnung & $\begin{array}{l}\text { Konstruktions- } \\
\text { empfehlung }\end{array}$ & Konsequenz & Erklärung \\
\hline $\begin{array}{l}\text { PIDG } \\
\text { Korrosions- } \\
\text { schutz }\end{array}$ & $\begin{array}{l}\text { Nutze in Gussbau- } \\
\text { teile eingegossene } \\
\text { Elemente mit einem } \\
\text { niedrigeren Ruhepo- } \\
\text { tential als das Mate- } \\
\text { rial des Gussbauteils } \\
\text { als Opferanoden. }\end{array}$ & $\begin{array}{l}\text { Korrosions- } \\
\text { schutz }\end{array}$ & $\begin{array}{l}\text { Bei Kontaktkorrosion in } \\
\text { korrosiver Umgebung } \\
\text { wird das Material mit } \\
\text { negativerem Ruhepo- } \\
\text { tential korrodieren. } \\
\text { (Vgl. Kaesche (2011).) }\end{array}$ \\
\hline $\begin{array}{l}\text { PIDG } \\
\text { Klemmverbin- } \\
\text { dung }\end{array}$ & $\begin{array}{l}\text { Nutze umformbe- } \\
\text { dingte Eigenspan- } \\
\text { nungen in spaltpro- } \\
\text { filierten Bauteilen, } \\
\text { um Verbindungsele- } \\
\text { mente zu klemmen. }\end{array}$ & $\begin{array}{l}\text { Verringerung } \\
\text { des Fügeauf- } \\
\text { wands }\end{array}$ & $\begin{array}{l}\text { Bohrungen in spaltpro- } \\
\text { filierten Bauteilen füh- } \\
\text { ren zum Freisetzen von } \\
\text { umformbedingten Ei- } \\
\text { genspannungen. (Vgl. } \\
\text { Wagner et al. (2017).) }\end{array}$ \\
\hline $\begin{array}{l}\text { PIDG } \\
\text { geschweißte } \\
\text { Flansche }\end{array}$ & $\begin{array}{l}\text { Positioniere } \\
\text { Schweißnähte an } \\
\text { den Enden spaltprofi- } \\
\text { lierter Flansche, um } \\
\text { Bauteilbereiche zu } \\
\text { verbinden. }\end{array}$ & $\begin{array}{l}\text { Erhalten der } \\
\text { hohen Härte } \\
\text { spaltprofilier- } \\
\text { ter Flansche }\end{array}$ & $\begin{array}{l}\text { Gefügeveränderungen } \\
\text { durch das Schweißen } \\
\text { sind an den Flanschen- } \\
\text { den minimal, da dort } \\
\text { nur eine geringfügig er- } \\
\text { höhte Härte vorliegt. } \\
\text { (Vgl. Ahmels et al. } \\
\text { (2017).) }\end{array}$ \\
\hline $\begin{array}{l}\text { PIDG } \\
\text { Schnappver- } \\
\text { bindung }\end{array}$ & $\begin{array}{l}\text { Nutze spaltprofi- } \\
\text { lierte Flansche mit } \\
\text { ihrer erhöhten Härte } \\
\text { als Füge- oder Hal- } \\
\text { teflächen einer } \\
\text { Schnappverbindung. }\end{array}$ & $\begin{array}{l}\text { Übertragun- } \\
\text { gen hoher } \\
\text { Kontaktkräfte }\end{array}$ & $\begin{array}{l}\text { Spaltprofilierte Flan- } \\
\text { sche zeichnen sich } \\
\text { durch eine erhöhte } \\
\text { Härte an der Flanscho- } \\
\text { berseite aus. (Vgl. Ah- } \\
\text { mels et al. (2017).) }\end{array}$ \\
\hline
\end{tabular}


Anhang D - Übersicht über die Arbeitsschritte der fertigungsintegrierenden Entwicklungsmethodik

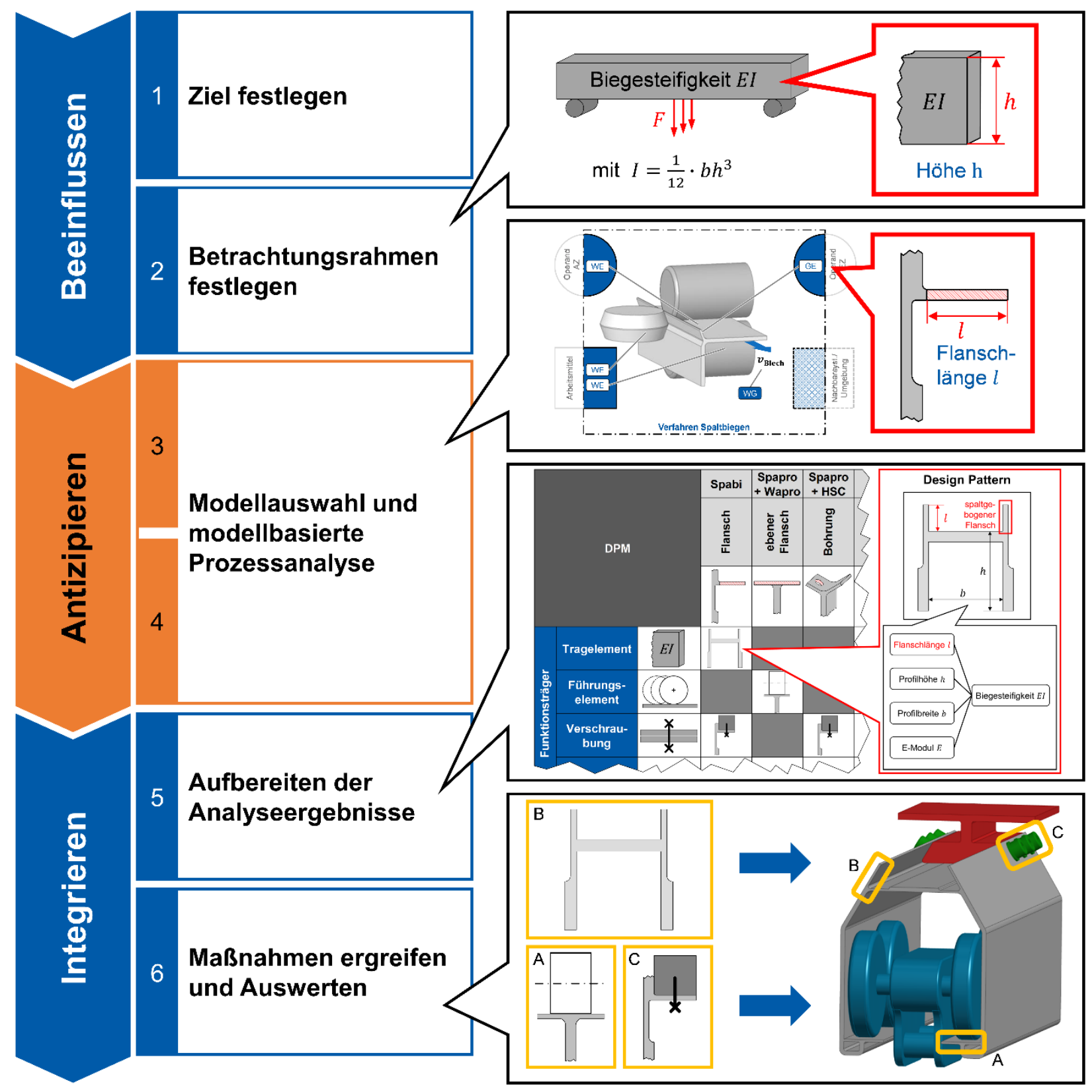




\section{Anhang E - Glossar}

Die Quellennachweise für die mit ${ }^{(L)}$ gekennzeichneten Begriffe sind jeweils in den Kapiteln 1 bis 7 zu finden.

\begin{tabular}{|c|c|}
\hline Begriff & Definition \\
\hline $\begin{array}{l}\text { Abhängige Produkt- } \\
\text { modelleigenschaft (L) }\end{array}$ & $\begin{array}{l}\text { Abhängige Produktmodelleigenschaften sind dieje- } \\
\text { nigen Produktmodelleigenschaften, die im Rahmen } \\
\text { eines Produktmodells nur indirekt durch Festlegen } \\
\text { von unabhängigen Produktmodelleigenschaften } \\
\text { festgelegt werden können. }\end{array}$ \\
\hline Antizipieren & $\begin{array}{l}\text { Das Antizipieren technischer Prozesse des Pro- } \\
\text { duktlebenslaufs ist eine zielgerichtete, auf das je- } \\
\text { weilige Konkretisierungsniveau angepasste mo- } \\
\text { dellbasierte Prozessanalyse ausgehend von be- } \\
\text { reits getätigten konstruktiven Festlegungen wäh- } \\
\text { rend des Produktentwicklungsprozesses. }\end{array}$ \\
\hline $\begin{array}{l}\text { Arbeitsmittel } \\
\text { (Operator) }^{(L)}\end{array}$ & $\begin{array}{l}\text { Das technische Produkt als Arbeitsmittel im techni- } \\
\text { schen Prozess stellt die benötigte }(n) \text { Wirkgröße(n) } \\
\text { bereit, die die Zustandsänderung des Operanden } \\
\text { realisieren. }\end{array}$ \\
\hline Eigenschaft ${ }^{(\mathrm{L})}$ & $\begin{array}{l}\text { Eine Eigenschaft beschreibt ein Objekt in Form ei- } \\
\text { nes Merkmals und einer zugehörigen Ausprägung, } \\
\text { wobei die Ausprägung einer merkmalspezifischen } \\
\text { Menge möglicher Ausprägungen entstammt. }\end{array}$ \\
\hline Eigenschaftsrelation (L) & $\begin{array}{l}\text { Eigenschaftsrelationen bilden die Abhängigkeiten } \\
\text { und Beziehungen zwischen Eigenschaften ab. }\end{array}$ \\
\hline Einsatzprozess ${ }^{(\mathrm{L})}$ & $\begin{array}{l}\text { Einsatzprozesse sind die Prozesse der Nutzungs- } \\
\text { phase, in denen das Produkt als Arbeitsmittel fun- } \\
\text { giert. }\end{array}$ \\
\hline $\begin{array}{l}\text { Fertigungsanaloger } \\
\text { Nutzungsprozess }(\mathrm{L})\end{array}$ & $\begin{array}{l}\text { Fertigungsanaloge Nutzungsprozessen sind die } \\
\text { Prozesse der Nutzungsphase, in denen das Pro- } \\
\text { dukt als Operand fungiert. }\end{array}$ \\
\hline $\begin{array}{l}\text { (Fertigungsintegrierende) } \\
\text { Design Pattern Matrix }\end{array}$ & $\begin{array}{l}\text { Die (fertigungsintegrierende) Design Pattern Matrix } \\
\text { dient der systematischen Gegenüberstellung und } \\
\text { Verknüpfung konkreter eigenschaftsbasierter Mo- } \\
\text { delle. In erster Linie wird sie verwendet, um ferti- } \\
\text { gungstechnologisches Produkt-Wissen mit weite- } \\
\text { rem Produkt-Wissen zu verknüpfen. }\end{array}$ \\
\hline
\end{tabular}




\begin{tabular}{ll}
\hline Begriff & Definition \\
\hline Fertigungsintegrierende & Fertigungsintegrierende Produktlösungen sind Lö- \\
Produktlösung (L) & sungen, die nicht nur die Erwartungen der Stake- \\
& holder bestmöglich erfüllen, sondern auch einen \\
& erkennbaren Mehrwert in Bezug auf die Erfüllung \\
& der Produktfunktion sowie die Fertigung und Mon- \\
& tage bieten, indem Fertigungspotenziale durch Zu- \\
& rückgreifen auf fertigungstechnologisches Wissen \\
& umfassend genutzt werden. \\
\hline
\end{tabular}

Fertigungsintegrierendes Design Pattern

Fertigungsintegrierende Design Patterns sind allgemeingültige Lösungselemente, die auf der Verknüpfung von Eigenschaften zur Produktfunktion und Eigenschaften zu einem Fertigungsverfahren beruhen. Sie setzen sich aus einem Modell (z. B. einer grafischen Repräsentation des Lösungselements) und einem wiederkehrenden Muster aus in Relation stehenden Eigenschaften (z. B. Eigenschaftsnetzwerke oder mathematische Formeln) zusammen.

Fertigungspotenziale Fertigungspotenziale resultieren aus ungenutzten Möglichkeiten einer Fertigungstechnologie. Die gezielte Ausschöpfung dieser Potenziale ermöglicht beispielsweise Verbesserungen in Bezug auf die Produktfunktion.

Fertigungstechnologie

Fertigungstechnologien umfassen Wissen über die Umwandlung und Transformation von Roh- und Werkstoffen in fertige Produkte. Durch neue Erkenntnisse wird dieses Wissen stetig erweitert.

Fertigungstechnologisches Wissen ${ }^{(L)}$
Das fertigungstechnologische Wissen beinhaltet allgemeingültige Aussagen, die $u$. a. aus Versuchen, analytischen Rechnungen, Beobachtungen oder Simulationen abgeleitet wurden und deren Relevanz für das Verständnis der Fertigungstechnologie belegt werden konnte.

Fügen $^{(\llcorner)}$

Fügen ist ein auf Dauer angelegtes Verbinden oder sonstiges Zusammenbringen von zwei oder mehreren Werkstücken geometrisch bestimmter fester Form oder von eben solchen Werkstücken mit formlosem Stoff; dabei wird der Zusammenhalt örtlich geschaffen und im Ganzen vermehrt.

Funktionsträger ${ }^{(\mathrm{L})}$
Funktionsträger sind konstruktionsbestimmende Elemente, die die Funktion des Produkts bestimmen. Funktionsträger können Teile, Lösungsprinzipien oder Prinziplösungen sein, die eine oder mehrere Funktionen erfüllen. 


\begin{tabular}{ll}
\hline Begriff & Definition \\
\hline Gestalteigenschaft (L) & Gestalteigenschaften umfassen die geometrischen \\
& und werkstofflichen Eigenschaften eines Produkts. \\
& Werkstoffliche Eigenschaften bzw. Eigenschaften, \\
& die vom verwendeten Material dominiert werden, \\
& umfassen zudem mechanische Eigenschaften, Ei- \\
& genschaften der Oberfläche und physikalische Ei- \\
& genschaften. Ergänzend können auch Eigenschaf- \\
& ten über den energetischen, informationstechni- \\
& schen sowie orts- und lagebezogenen Zustand er- \\
& fasst werden. \\
\hline
\end{tabular}

Gestaltelement (L) Gestaltelemente sind die Elemente, in die sich die Produktgestalt zerlegen und damit strukturieren lässt. Sie werden mittels Gestalteigenschaften geometrisch und werkstofflich beschrieben.

\begin{tabular}{|c|c|}
\hline Größe ${ }^{(L)}$ & $\begin{array}{l}\text { Größen - im Sinne von Prozessgrößen - beschrei- } \\
\text { ben keinen Zustand eines Objekts, sondern Ener- } \\
\text { gien und Signale die zwischen den Objekten aus- } \\
\text { getauscht werden. Sie setzen sich aus einem } \\
\text { Merkmal und einer zugehörigen Ausprägung zu- } \\
\text { sammen. }\end{array}$ \\
\hline Lösungselement & $\begin{array}{l}\text { Lösungselemente stellen noch keine vollwertigen } \\
\text { Lösungen eines technischen Problems dar. Sie } \\
\text { sind vielmehr allgemeingültige Bestandteile poten- } \\
\text { zieller technischer Lösungen. Im Rahmen der pro- } \\
\text { jektspezifischen Lösungsfindung können die Lö- } \\
\text { sungselemente für die Generierung von Varianten } \\
\text { genutzt werden, aus denen die eigentliche techni- } \\
\text { sche Lösung hervorgeht. }\end{array}$ \\
\hline Mapping (L) & $\begin{array}{l}\text { Als (Eigenschafts-) Mapping wird der systemati- } \\
\text { sche Abgleich von verfahrensinduzierten Eigen- } \\
\text { schaften mit wirkprinziprelevanten und verfahrens- } \\
\text { prinziprelevanten Eigenschaften verstanden. }\end{array}$ \\
\hline Merkmal (L) & $\begin{array}{l}\text { Ein Merkmal und eine zugeordnete Ausprägung } \\
\text { stellen die elementaren Bestandteile einer Eigen- } \\
\text { schaft dar. }\end{array}$ \\
\hline Methode (L) & $\begin{array}{l}\text { Eine Methode beschreibt ein planmäßiges, regel- } \\
\text { basiertes Vorgehen unter Angabe bestimmter Ar- } \\
\text { beitsschritte, mit deren Hilfe ein zuvor definiertes } \\
\text { Ziel erreicht werden soll. Eine Methode gibt dazu } \\
\text { Empfehlungen oder Anweisungen, die bei der } \\
\text { Durchführung der Arbeitsschritte und der Doku- } \\
\text { mentation der Ergebnisse helfen sollen. }\end{array}$ \\
\hline
\end{tabular}




\begin{tabular}{|c|c|}
\hline Begriff & Definition \\
\hline Methodik (L) & $\begin{array}{l}\text { Eine Methodik beschreibt ein planmäßiges Vorge- } \\
\text { hen, in das mehrere Methoden und Werkzeuge } \\
\text { einbezogen werden. }\end{array}$ \\
\hline Modell (L) & $\begin{array}{l}\text { Modelle sind vereinfachte abstrahierte Abbilder, } \\
\text { Darstellungen oder gedankliche oder stoffliche Ge- } \\
\text { bilde der komplexen Realität mit einer dem Zweck } \\
\text { der Modellbildung entsprechenden Genauigkeit. }\end{array}$ \\
\hline Montieren (L) & $\begin{array}{l}\text { Montieren wird als die Gesamtheit aller Vorgänge } \\
\text { verstanden, die dem Zusammenbau von geomet- } \\
\text { risch bestimmten Körpern dienen. }\end{array}$ \\
\hline Objekt (L) & Objekte sind geometrisch stoffliche Gebilde. \\
\hline Operand $^{(\mathrm{L})}$ & $\begin{array}{l}\text { Operanden durchlaufen im Rahmen technischer } \\
\text { Prozesse eine Zustandsänderung von einem An- } \\
\text { fangs- in einen Endzustand. Als Operanden fungie- } \\
\text { ren ausschließlich Objekte, also stoffliche Gebilde. }\end{array}$ \\
\hline Physikalischer Effekt (L) & $\begin{array}{l}\text { Physikalische Effekte beruhen auf physikalischen } \\
\text { Gesetzmäßigkeiten. Entsprechendes gilt auch für } \\
\text { biologische und chemische Effekte. Sie können zur } \\
\text { Konkretisierung der technischen Lösung im Rah- } \\
\text { men der Produkt- und Prozessmodellierung ver- } \\
\text { wendet werden. }\end{array}$ \\
\hline $\begin{array}{l}\text { Process Integrated } \\
\text { Design Guideline }\end{array}$ & $\begin{array}{l}\text { Process Integrated Design Guidelines geben in } \\
\text { Anknüpfung an die fertigungsintegrierenden De- } \\
\text { sign Patterns an, wie verfahrensinduzierte Gestalt- } \\
\text { elemente und deren Eigenschaften zur Realisie- } \\
\text { rung bzw. Erweiterung der Produktfunktion beitra- } \\
\text { gen können. Sie setzen sich aus Bezeichnung, } \\
\text { Konstruktionsempfehlung, Konsequenz und Erklä- } \\
\text { rung zusammen. }\end{array}$ \\
\hline Produkteigenschaft (L) & $\begin{array}{l}\text { Eine Eigenschaft, die ein technisches Produkt be- } \\
\text { schreibt, wir als Produkteigenschaft bezeichnet. }\end{array}$ \\
\hline Produktfunktion (L) & $\begin{array}{l}\text { Die Produktfunktion ist eine lösungsneutrale Be- } \\
\text { schreibung des kausalen Zusammenhangs zwi- } \\
\text { schen gewollten Ein- und Ausgangsgrößen des } \\
\text { technischen Produkts. Als Ein- und Ausgangsgrö- } \\
\text { ßen fungieren ausschließlich Energien und Sig- } \\
\text { nale. }\end{array}$ \\
\hline Produktgestalt (L) & $\begin{array}{l}\text { Die Produktgestalt wird durch die Summe der un- } \\
\text { abhängigen Gestalteigenschaften eines techni- } \\
\text { schen Produkts beschrieben. }\end{array}$ \\
\hline Produktlebenslauf (L) & $\begin{array}{l}\text { Der Produktlebenslauf gliedert sich in die vier Le- } \\
\text { benslaufphasen Werkstoffherstellung, Produktion, } \\
\text { Nutzung und Recycling/Entsorgung. }\end{array}$ \\
\hline
\end{tabular}




\begin{tabular}{|c|c|}
\hline Begriff & Definition \\
\hline Produktmodell (L) & $\begin{array}{l}\text { Produktmodelle werden als vereinfachte abstra- } \\
\text { hierte Abbilder des realen Produkts verstanden. } \\
\text { Sie bilden alle produktdefinierenden Informationen } \\
\text { ab, die während des Produktentwicklungsprozes- } \\
\text { ses festgelegt werden. }\end{array}$ \\
\hline $\begin{array}{l}\text { Produktmodell- } \\
\text { eigenschaft }(\mathrm{L})\end{array}$ & $\begin{array}{l}\text { Produkteigenschaften, die das Produkt im Kontext } \\
\text { eines spezifischen Produktmodells beschreiben, } \\
\text { werden Produktmodelleigenschaften bezeichnet. }\end{array}$ \\
\hline $\begin{array}{l}\text { Produktmodell- } \\
\text { pyramide }^{(L)}\end{array}$ & $\begin{array}{l}\text { Die Produktmodellpyramide umfasst aufeinander } \\
\text { abgestimmte Produktmodelle, sogenannte Partial- } \\
\text { modelle, die verschiedenen Ebenen der Produkt- } \\
\text { modellpyramide zugeordnet sind. Der Aufbau der } \\
\text { Produktmodellpyramide korreliert mit dem Vorge- } \\
\text { hen während der Produktentwicklung. Die Komple- } \\
\text { xität und der Konkretisierungsgrad nehmen in } \\
\text { Richtung der unteren Ebenen zu. }\end{array}$ \\
\hline Prozesseigenschaft & $\begin{array}{l}\text { Prozesseigenschaften umfassen Eigenschaften } \\
\text { von am technischen Prozess beteiligten Objekten, } \\
\text { die jedoch nicht einem einzelnen Objekt in einem } \\
\text { konkreten Zustand vollständig zugeordnet werden } \\
\text { können. Dennoch sind sie für eine vollständige Be- } \\
\text { schreibung der Wirkzusammenhänge im Kontext } \\
\text { eines Verfahrens unabdingbar. }\end{array}$ \\
\hline Prozessmodell (L) & $\begin{array}{l}\text { Prozessmodelle stellen zweckdienliche Abbildun- } \\
\text { gen von Prozessen dar. }\end{array}$ \\
\hline
\end{tabular}

Technischer Prozess (L) Ein technischer Prozess beschreibt eine Zustandsänderung eines oder mehrerer Operanden in einem Zeitintervall. Der oder die Operanden werden dabei von einem Anfangszustand in einen Endzustand transformiert. Als Operanden fungieren ausschließlich Objekte, also stoffliche Gebilde.

Technisches Produkt (L) Technische Produkte werden als technische Systeme oder Bestandteile übergeordneter technischer Systeme verstanden und beschrieben.

Transformation/ Transfor- Transformationsprozesse beschreiben die Zumationsprozess ${ }^{(L)} \quad$ standsänderung eines Operanden. Die Transformation ist mit der Zustandsänderung gleichbedeutend.

Unabhängige Produktmodelleigenschaft (L)
Unabhängige Produktmodelleigenschaften sind diejenigen Produktmodelleigenschaften, die im Rahmen eines Produktmodells unabhängig von anderen Eigenschaften festgelegt werden können. 


\begin{tabular}{|c|c|}
\hline Begriff & Definition \\
\hline Verfahren (L) & $\begin{array}{l}\text { Verfahren beschreiben die geordnete Abfolge von } \\
\text { Operationen, die notwendig sind, um einen Pro- } \\
\text { zess zu realisieren. Verfahren definieren, in wel- } \\
\text { cher Art und Weise die Zustandsänderung abläuft. }\end{array}$ \\
\hline $\begin{array}{l}\text { Verfahrensinduzierte } \\
\text { Eigenschaft (L) }\end{array}$ & $\begin{array}{l}\text { Verfahrensinduzierte Eigenschaften sind Gestaltei- } \\
\text { genschaften, die die verfahrensinduzierten Gestalt- } \\
\text { elemente des Operanden im Endzustand des tech- } \\
\text { nischen Prozesses beschreiben. Sie werden cha- } \\
\text { rakteristischerweise durch ein bestimmtes Verfah- } \\
\text { ren hervorgerufen. }\end{array}$ \\
\hline $\begin{array}{l}\text { Verfahrensinduziertes } \\
\text { Gestaltelement }(\mathrm{L})\end{array}$ & $\begin{array}{l}\text { Verfahrensinduzierte Gestaltelemente sind die Ge- } \\
\text { staltelemente des oder der Operanden des techni- } \\
\text { schen Prozesses, die durch ein bestimmtes Ver- } \\
\text { fahren hervorgerufen werden. }\end{array}$ \\
\hline Verfahrensprinzip (L) & $\begin{array}{l}\text { Das Verfahrensprinzip eines Verfahrens beschreibt } \\
\text { den Wirkzusammenhang zwischen Wirkelementen } \\
\text { und Wirkgrößen, die an der Realisierung der Zu- } \\
\text { standsänderung des Operanden beteiligt sind. }\end{array}$ \\
\hline $\begin{array}{l}\text { Verfahrensprinzip- } \\
\text { relevante Eigenschaft (L) }\end{array}$ & $\begin{array}{l}\text { Verfahrensprinziprelevante Eigenschaften umfas- } \\
\text { sen alle Eigenschaften, die die verfahrensprinzip- } \\
\text { relevanten Wirkelemente beschreiben. }\end{array}$ \\
\hline $\begin{array}{l}\text { Verfahrensprinzip- } \\
\text { relevantes Wirkelement }(\text { (L) }\end{array}$ & $\begin{array}{l}\text { Verfahrensprinziprelevante Wirkelemente umfas- } \\
\text { sen Wirkflächen, Wirkkörper oder Wirkräume des } \\
\text { Operanden oder des Arbeitsmittels des techni- } \\
\text { schen Prozesses, die im Rahmen eines Verfah- } \\
\text { rens in einem Wirkzusammenhang stehen. Sie } \\
\text { werden durch verfahrensprinziprelevante Eigen- } \\
\text { schaften beschrieben. }\end{array}$ \\
\hline Vorgehensmodell (L) & $\begin{array}{l}\text { Vorgehensmodelle setzen sich aus aufeinanderfol- } \\
\text { genden oder parallelisierten Schritten bzw. Aktivi- } \\
\text { täten zusammen, die während der Entwicklung } \\
\text { durchlaufen werden. }\end{array}$ \\
\hline Wirkflächenpaar ${ }^{(L)}$ & $\begin{array}{l}\text { Wirkflächen dienen in erster Linie der Übertragung } \\
\text { von Wirkgrößen (Energien und Signale). Die Wirk- } \\
\text { fläche steht dabei meist in Interaktion mit einer } \\
\text { zweiten Wirkfläche. Eine solche Kombination wird } \\
\text { als Wirkflächenpaar bezeichnet. }\end{array}$ \\
\hline Wirkgröße ${ }^{(L)}$ & $\begin{array}{l}\text { Wirkgrößen wirken im Rahmen eines technischen } \\
\text { Prozesses auf den Operanden ein, um dessen Zu- } \\
\text { standsänderung einzuleiten und voranzutreiben. } \\
\text { Als Wirkgrößen fungieren ausschließlich Energien } \\
\text { oder Signale. }\end{array}$ \\
\hline
\end{tabular}




\begin{tabular}{ll}
\hline Begriff & Definition \\
\hline Wirkprinzip (L) & $\begin{array}{l}\text { Das Wirkprinzip bringt Wirkelemente in einen Wirk- } \\
\text { zusammenhang, indem erste geometrische und } \\
\text { werkstoffliche Eigenschaften festgelegt werden. Es } \\
\text { trägt zur Konkretisierung der Produktfunktion bei. }\end{array}$ \\
\hline $\begin{array}{l}\text { Wirkprinziprelevante } \\
\text { Eigenschaft (L) }\end{array}$ & $\begin{array}{l}\text { Wirkprinziprelevante Eigenschaften, sind die Pro- } \\
\text { duktmodelleigenschaften, die für die Umsetzung } \\
\text { des Wirkprinzips unabdingbar sind. }\end{array}$ \\
\hline Zustand (L) & $\begin{array}{l}\text { Jeder Zustand eines Objekts kann zu bestimmten } \\
\text { Zeitpunkten über eine Menge von Eigenschaften } \\
\text { beschrieben werden. }\end{array}$ \\
\hline
\end{tabular}




\section{Literaturverzeichnis}

Abele et al. (2008)

Abele, E.; Anderl, R.; Birkhofer, H.; Rüttinger, B.: EcoDesign. Von der Theorie in die Praxis. Berlin - New York: Springer Verlag 2008.

\section{Ahmels et al. (2017)}

Ahmels, L.; Bott, A.-K.; Bruder, E.; Gibbels, M.; Gramlich, S.; Hansmann, M.; Karin, I.; Kohler, M.; Lipp, K.; Melz, T.; Müller, C.; Neufeld, D.; Niehuesbernd, J.; Roos, M.; Tomasella, A.; Ulbrich, S.; Wagener, R.; Walter, A.: Manufacturing Induced Properties: Determination, Understanding, and Beneficial Use. In: Groche, P.; Bruder, E.; Gramlich, S. (Hrsg.): Manufacturing Integrated Design. Sheet Metal Product and Process Innovation. Cham: Springer International Publishing 2017, S. 99-145.

\section{Altan, Miller (1990)}

Altan, T.; Miller, R. A.: Design for Forming and other Near Net Shape Manufacturing Processes. In: CIRP Annals - Manufacturing Technology, 39 (2). 1990, S. 609-620. https://doi.org/10.1016/S0007-8506(07)62998-9.

\section{Anderl, Trippner (2000)}

Anderl, R.; Trippner, D.: STEP STandard for the Exchange of Product Model Data. Eine Einführung in die Entwicklung, Implementierung und industrielle Nutzung der Normenreihe ISO 10303 (STEP). Wiesbaden: Vieweg+Teubner Verlag 2000. https://doi.org/10.1007/978-3-322-89096-2.

\section{Anderson (2014)}

Anderson, D. M.: Design for Manufacturability. How to Use Concurrent Engineering to Rapidly Develop Low-Cost, High-Quality Products for Lean Production. Portland: CRC Press 2014.

\section{Andreasen et al. (1985)}

Andreasen, M. M.; Kähler, S.; Lund, T.: Montagegerechtes Konstruieren. Übers. aus d. Engl. von Laschet, Z. u. Duda, A. Berlin u. a.: Springer Verlag 1985.

\section{Andreasen, Hein (1987)}

Andreasen, M. M.; Hein, L.: Integrated Product Development. Berlin u. a.: Springer Verlag 1987. 
Ashby (2010)

Ashby, M. F.: Materials Selection in Mechanical Design. 4. ed. Amsterdam:

Butterworth-Heinemann 2010.

\section{Baehr (2005)}

Baehr, H. D.: Thermodynamik. Grundlagen und technische Anwendungen. 12., neu bearb. und erw. Aufl. Springer-Lehrbuch. Berlin u. a.: Springer 2005.

\section{Bahn et al. (2007)}

Bahn, V.; Bräunlich, H.; Hausstädtler, U.; Kurka, P.; Lachmann, L.; Lorenz, U.; Mauermann, R.; Michael, D.; Seifert, M.; Sterzing, A.; Weiser, M.:

Prozessgestaltung. In: Neugebauer, R. (Hrsg.): Hydro-Umformung. Berlin u. a.: Springer Verlag 2007, S. 33-162.

\section{Bauer (2007)}

Bauer, S.: Konzept und Umsetzung eines Systems zur strukturierten Sammlung und Bereitstellung von DfX-Richtlinien. In: Meerkamm, H. (Hrsg.): Proceedings of the $18^{\text {th }}$ Symposium on Design for X. DfX 2007. Neukirchen: 2007, S. 13-22.

\section{Bauer, Althof (1991)}

Bauer, C.-O.; Althof, W.: Handbuch der Verbindungstechnik. München - Wien: Hanser 1991.

\section{Birkhofer (1980)}

Birkhofer, H.: Analyse und Synthese der Funktionen technischer Produkte.

Fortschr.-Ber. VDI Reihe 1, Nr. 70. Düsseldorf: VDI-Verlag 1980. Dissertation.

\section{Birkhofer (2005)}

Birkhofer, H.: In fünf Minuten von der Aufgabe zur optimalen Lösung - ein Beitrag zur Algorithmisierung der frühen Phasen. In: Meerkamm, H. (Hrsg.): Proceedings of the $16^{\text {th }}$ Symposium on Design for X. DfX 2005. Neukirchen: 2005, S. 47-58.

\section{Birkhofer (2011)}

Birkhofer, $\mathrm{H} .:$ From design practice to design science: the evolution of a career in design methodology research. In: Journal of Engineering Design, 22 (5). 2011, S. 333-359. https://doi.org/10.1080/09544828.2011.555392.

\section{Birkhofer, Nordmann (2002)}

Birkhofer, H.; Nordmann, R.: Maschinenelemente und Mechatronik II. 2., überarb. Aufl. Studienskripte. Aachen: Shaker Verlag 2002. 


\section{Birkhofer, Schott (1996)}

Birkhofer, H.; Schott, H.: Die Entwicklung umweltgerechter Produkte - eine Herausforderung für die Konstruktionswissenschaft. In: Konstruktion, 48 (12). 1996, S. 386-396.

\section{Birkhofer, Wäldele (2005)}

Birkhofer, H.; Wäldele, M.: Applied Engineering Design Science - The Missing Link between Design Science and Design in Industry. In: Hosnedl, S. (Hrsg.):

Proceedings of the Applied Engineering Design Science Workshop. AEDS 2005.

Pilsen, Tschechische Republik: 2005.

\section{Birkhofer, Wäldele (2008)}

Birkhofer, H.; Wäldele, M.: Properties and characteristics and attributes and... - an approach on structuring the description of technical systems. In: Vanek, V.; Hosnedl, S.; Bartak, J. (Hrsg.): Proceedings of the Applied Engineering Design Science Workshop. AEDS 2008. Pilsen, Tschechische Republik: 2008, S. 19-34.

\section{Birkhofer et al. (2007)}

Birkhofer, H.; Anderl, R.; Franke, H.-J.: Life Cycle Engineering. In: Krause, F.-L.; Gausemeier, J. (Hrsg.): Innovationspotenziale in der Produktentwicklung. München - Wien: Hanser Verlag 2007, S. 205-215.

\section{Birkhofer et al. (2012)}

Birkhofer, H.; Rath, K.; Zhao, S.: Umweltgerechtes Konstruieren. In: Rieg, F.;

Steinhilper, R. (Hrsg.): Handbuch Konstruktion. München - Wien: Hanser Verlag 2012, S. 563-581.

\section{Boothroyd et al. (2010)}

Boothroyd, G.; Dewhurst, P.; Knight, W. A.: Product Design for Manufacture and Assembly. $3^{\text {rd }}$ ed. Boca Raton: CRC Press 2010.

\section{Bralla (1999)}

Bralla, J. G.: Design for Manufacturability Handbook. 2. ed. Boston, Massachusetts u. a.: McGraw-Hill 1999.

\section{Bruder (2011)}

Bruder, E.: Thermische Stabilität von Stählen mit ultrafeinkörnigen

Gradientengefügen und deren mechanische Eigenschaften. 2011. Dissertation. http://tuprints.ulb.tu-darmstadt.de/id/eprint/2415. 


\section{Dangelmaier (2003)}

Dangelmaier, W.: Produktion und Information. Berlin - Heidelberg: Springer Verlag 2003. https://doi.org/10.1007/978-3-642-55584-8.

\section{Dannheim (1999)}

Dannheim, F.: Die Entwicklung umweltgerechter Produkte im Spannungsfeld von Ökologie und Ökonomie. Eine Analyse unter besonderer Berücksichtigung der Nutzungsphase. Fortschr.-Ber. VDI Reihe 1, Nr. 320. Düsseldorf: VDI-Verlag 1999. Dissertation.

\section{Denkena, Tönshoff (2011)}

Denkena, B.; Tönshoff, H. K.: Spanen. Grundlagen. 3., bearb. und erw. Aufl. VDI. Berlin: Springer Verlag 2011. https://doi.org/10.1007/978-3-642-19772-7.

\section{DVS 2242-1 (2011)}

DVS 2242-1:2011-04, Mechanisches Fügen von Kunststoffbauteilen Schnappverbindungen. Deutscher Verband für Schweißen und verwandte Verfahren e. V. (Hrsg.). Düsseldorf: DVS Media 2011.

DIN 8580 (2003)

DIN 8580:2003-09, Fertigungsverfahren - Begriffe, Einleitung. Deutsches Institut für Normung e. V. (Hrsg.). Berlin: Beuth Verlag 2003.

\section{DIN 8593-1 (2003)}

DIN 8593-1:2003-09, Fertigungsverfahren Fügen - Teil 1: Zusammensetzen Einordnung, Unterteilung, Begriffe. Deutsches Institut für Normung e. V. (Hrsg.). Berlin: Beuth Verlag 2003.

\section{DIN EN ISO 9000 (2015)}

DIN EN ISO 9000:2015-11, Qualitätsmanagementsysteme - Grundlagen und Begriffe. Deutsches Institut für Normung e. V. (Hrsg.). Berlin: Beuth Verlag 2015.

\section{DIN EN ISO 14040 (2009)}

DIN EN ISO 14040:2009-11, Umweltmanagement - Ökobilanz - Grundsäze und Rahmenbedingungen. Deutsches Institut für Normung e. V. (Hrsg.). Beuth Verlag 2009 .

\section{Dilthey (1994)}

Dilthey, U.: Schweißtechnische Fertigungsverfahren. Schweiß- und Schneidtechnologien. Zweite Aufl. Studium und Praxis. Berlin - Heidelberg: Springer 1994. https://doi.org/10.1007/978-3-662-12982-1. 


\section{Dudenredaktion (2017a)}

Dudenredaktion (o. J.): „Technologie“ auf Duden online. 2017.

http://www.duden.de/node/725198/revisions/1391929/view, zuletzt geprüft am 11.04.2017.

\section{Dudenredaktion (2017b)}

Dudenredaktion (o. J.): „Verfahren“ auf Duden online. 2017.

http://www.duden.de/node/663077/revisions/1318591/view, zuletzt geprüft am 07.04.2017.

\section{Ehrlenspiel (2009)}

Ehrlenspiel, K.: Integrierte Produktentwicklung. Denkabläufe, Methodeneinsatz, Zusammenarbeit. 4., aktualisierte Aufl. München - Wien: Hanser Verlag 2009.

\section{Ehrlenspiel, Meerkamm (2013)}

Ehrlenspiel, K.; Meerkamm, H.: Integrierte Produktentwicklung. Denkabläufe, Methodeneinsatz, Zusammenarbeit. 5., überarbeitete und erw. Aufl. München Wien: Hanser Verlag 2013. https://doi.org/10.3139/9783446436275.

\section{Feldhusen (2008)}

Feldhusen, J.: Gestaltung von Elementen und Systemen. In: Steinhilper, W.; Sauer,

B. (Hrsg.): Konstruktionselemente des Maschinenbaus 1. Grundlagen der Berechnung und Gestaltung von Maschinenelementen. 7. Aufl. Berlin - Heidelberg: Springer Verlag 2008, S. 163-198.

\section{Feldhusen, Grote (2013)}

Feldhusen, J.; Grote, K.-H.: Pahl/Beitz Konstruktionslehre. Methoden und Anwendung erfolgreicher Produktentwicklung. 8. überarbeitete Aufl. Berlin Heidelberg: Springer Verlag 2013. https://doi.org/10.1007/978-3-642-29569-0.

\section{Flach (1994)}

Flach, W.: Grundzüge der Erkenntnislehre. Erkenntniskritik, Logik, Methodologie. Würzburg: Königshausen und Neumann 1994.

\section{Franke (1976)}

Franke, H.-J.: Untersuchungen zur Algorithmisierbarkeit des

Konstruktionsprozesses. Fortschr.-Ber. VDI Reihe 1, Nr. 47. Düsseldorf: VDI-Verlag 1976. Dissertation.

\section{Fritz, Schulze (2010)}

Fritz, A. H.; Schulze, G.: Fertigungstechnik. Berlin - Heidelberg: Springer Verlag 2010. https://doi.org/10.1007/978-3-642-12879-0. 


\section{Gamma (2007)}

Gamma, E.: Design Patterns. Elements of Reusable Object-Oriented Software. 39th printing. Addison-Wesley professional computing series. Boston u. a.: AddisonWesley 2007.

\section{Grabowski et al. (1993)}

Grabowski, H.; Anderl, R.; Polly, A.; Warnecke, H.-J. (Hrsg.): Integriertes

Produktmodell. 1. Aufl. Entwicklungen zur Normung von CIM. Berlin u. a.: Beuth Verlag 1993.

\section{Gramlich (2013)}

Gramlich, S.: Vom fertigungsgerechten Konstruieren zum produktionsintegrierenden Entwickeln. Durchgängige Modelle und Methoden im Produktlebenszyklus. Fortschr.-Ber. VDI Reihe 1, Nr. 423. Düsseldorf: VDI-Verlag 2013. Dissertation.

\section{Gramlich et al. (2011)}

Gramlich, S.; Birkhofer, H.; Bohn, A.: Design Process Automation - a structured product description by properties and development of optimization algorithms. In: Culley, S. J.; Hicks, B. J.; McAloone, T.C.; Howard, T.J.; Clarkson, P. J. (Hrsg.): Proceedings of the $18^{\text {th }}$ International Conference on Engineering Design. ICED 2011. Vol. 1. Kopenhagen, Dänemark: 2011, S. 299-309.

\section{Gramlich et al. (2015)}

Gramlich, S.; Roos, M.; Ahmels, L.; Kaune, V.; Müller, C.; Bauer, O.; Karin, I.;

Tomasella, A.; Melz, T.: Ein wissensbasierter fertigungsintegrierender

Produktentwicklungsansatz. In: Binz, H.; Bertsche, B.; Bauer, W.; Roth, D. (Hrsg.):

Stuttgarter Symposium für Produktentwicklung 2015. Entwicklung smarter Produkte für die Zukunft. SSP 2015. Stuttgart: 2015.

\section{Groche et al. (2007)}

Groche, P.; Vucic, D.; Jöckel, M.: Basics of linear flow splitting. In: Journal of Materials Processing Technology, 183 (2). 2007, S. 249-255.

https://doi.org/10.1016/j.jmatprotec.2006.10.023.

\section{Groche et al. (2010)}

Groche, P.; Müller, C.; Beiter, P.; Ludwig, C.; Schmitt, W.; Ibis, M.: Future trends in cold rolled profile process technology. In: Confederation of British Metalforming: cbm, 19. 2010, S. 16-19. 


\section{Groche et al. (2012a)}

Groche, P.; Schmitt, W.; Bohn, A.; Gramlich, S.; Ulbrich, S.; Günther, U.: Integration of manufacturing-induced properties in product design. In: CIRP Annals Manufacturing Technology, 61 (1). 2012, S. 163-166.

https://doi.org/10.1016/j.cirp.2012.03.041.

\section{Groche et al. (2012b)}

Groche, P.; Birkhofer, H.; Bauer, O.; Göllner, T.; Gramlich, S.; Kaune, V.; Rullmann, F.; Weitzmann, O.: Potenziale einer durchgängigen Produktentstehung - Nutzung technologieinduzierter Eigenschaften zur Entwicklung von Blechstrukturen. In: Konstruktion (11/12). 2012, S. 85-90.

\section{Grote et al. (2008)}

Grote, K.-H.; Engelmann, F.; Beitz, W.; Syrbe, M.; Beyerer, J.: Entwicklung und Konstruktion. In: Czichos, H.; Hennecke, M. (Hrsg.): Hütte. Das Ingenieurwissen. 33., aktualisierte Aufl. Berlin - Heidelberg: Springer Verlag 2008, S. K1-K104.

\section{Grüner (2001)}

Grüner, C.: Die strategiebasierte Entwicklung umweltgerechter Produkte.

Konstruktionstechnik / Maschinenelemente, Nr. 349. Düsseldorf: VDI-Verlag 2001. Dissertation.

\section{Habenicht (2002)}

Habenicht, G.: Kleben. Grundlagen, Technologien, Anwendung. 4., erw. Auflage. VDI-Buch. Berlin - Heidelberg: Springer Verlag 2002. https://doi.org/10.1007/978-3662-08085-6.

\section{Hartl (1999)}

Hartl, C.: Theoretische Grundlagen der IHU. In: Siegert, K. (Hrsg.):

Hydroumformung von Rohren, Strangpreßprofilen und Blechen. Vortragstexte des Symposiums Internationale Konferenz Hydroumformung. Vol. 1. Fellbach bei Stuttgart. Frankfurt am Main: 1999, S. 23-35.

\section{Heidemann (2001)}

Heidemann, B.: Trennende Verknüpfung - Ein Prozessmodell als Quelle für Produktideen. Fortschr.-Ber. VDI Reihe 1, Nr. 351. Düsseldorf: VDI-Verlag 2001. Dissertation. 
Herrmann et al. (2004)

Herrmann, J. W.; Cooper, J.; Gupta, S. K.; Hayes, C. C.; Ishii, K.; Kazmer, D.; Sandborn, P. A.; Wood, W. H.: New Directions in Design for Manufacturing. In: $8^{\text {th }}$ Design for Manufacturing Conference. ASME 2004. Salt Lake City, Utah, USA: 2004, S. 853-861. https://doi.org/10.1115/DETC2004-57770.

\section{Hofmann, Spindler (2014)}

Hofmann, H.; Spindler, J.: Verfahren in der Beschichtungs- und Oberflächentechnik. 3., überarb. Aufl. München: Hanser Verlag 2014. https://doi.org/10.3139/9783446441835.

\section{Hubka (1984)}

Hubka, V.: Theorie technischer Systeme. Grundlagen einer wissenschaftlichen Konstruktionslehre. 2., völlig neubearb. und erw. Aufl. Berlin u. a.: Springer Verlag 1984.

ISO 10303-1 (1994)

ISO 10303-1:1994-12, Industrial automation systems and integration - Product data representation and exchange - Part 1: Overview and fundamental principles.

ISO/TC 184/SC 4 - American National Standards Institute (ANSI) (Hrsg.). Berlin: Beuth Verlag 1994.

Jaroschek (2013)

Jaroschek, C.: Spritzgießen für Praktiker. 3. Aufl. München: Hanser Verlag 2013. https://doi.org/10.3139/9783446436107.

\section{Jöckel (2005)}

Jöckel, M.: Grundlagen des Spaltprofilierens von Blechplatinen. Berichte aus Produktion und Umformtechnik, Nr. 64. Aachen: Shaker Verlag 2005. Dissertation.

\section{Johannaber, Michaeli (2004)}

Johannaber, F.; Michaeli, W.: Handbuch Spritzgießen. 2. Aufl. München: Hanser Verlag 2004. https://doi.org/10.3139/9783446440982.

\section{Kaesche (2011)}

Kaesche, H.: Die Korrosion der Metalle. Physikalisch-chemische Prinzipien und aktuelle Probleme. Klassiker der Technik. Berlin - Heidelberg: Springer Verlag 2011.

\section{Kesselring (1954)}

Kesselring, F.: Technische Kompositionslehre. Anleitung zu technischwirtschaftlichem und verantwortungsbewußtem Schaffen. Berlin - Heidelberg: Springer Verlag 1954. https://doi.org/10.1007/978-3-642-92624-2. 


\section{Kieffer, Hotop (1948)}

Kieffer, R.; Hotop, W.: Pulvermetallurgie und Sinterwerkstoffe. Zweite, verb. Aufl.

Reine und angewandte Metallkunde in Einzeldarstellungen, Nr. 9. Berlin -

Heidelberg: Springer Verlag 1948. https://doi.org/10.1007/978-3-642-94557-1.

\section{Klocke, König (2006)}

Klocke, F.; König, W.: Fertigungsverfahren 4: Umformen. 5., neu bearb. Aufl. VDI-

Buch. Berlin, Heidelberg: Springer Verlag 2006.

\section{Kögl, Moser (1981)}

Kögl, B.; Moser, F.: Grundlagen der Verfahrenstechnik. Vienna: Springer Verlag 1981. https://doi.org/10.1007/978-3-7091-2270-9.

\section{Koller (1994)}

Koller, R.: Konstruktionslehre für den Maschinenbau. Grundlagen zur Neu- und

Weiterentwicklung technischer Produkte. 3., völlig neubearb. und erw. Aufl.

Springer-Lehrbuch. Berlin - Heidelberg: Springer Verlag 1994.

\section{Kunz (1998)}

Kunz, C.: Neue Wege bei der Innenhochdruckumformung von stranggepreßten Aluminiumprofilen. Zürich: 1998. Dissertation.

\section{Lake (2016)}

Lake, M.: Oberflächentechnik in der Kunststoffverarbeitung. Vorbehandeln, beschichten, bedrucken, funktionalisieren, prüfen. 2., akt. Auflage. München: Hanser Verlag 2016.

\section{Leinemann (1974)}

Leinemann, K.: Beschreibung von Konstruktionsobjekten für den Rechnereinsatz. In: Chemie Ingenieur Technik, 46 (10). 1974, S. 439.

\section{Leyer (1963)}

Leyer, A.: Maschinenkonstruktionslehre. Allgemeine Gesichtspunkte. Technica-

Reihe, Nr. 1. Basel - Stuttgart: Birkhäuser Verlag 1963.

\section{Lindemann (2009)}

Lindemann, U.: Methodische Entwicklung technischer Produkte. Methoden flexibel und situationsgerecht anwenden. 3., korr. Aufl. Berlin - Heidelberg: Springer Verlag 2009. 


\section{Lommatzsch et al. (2011)}

Lommazsch, N.; Gramlich, S.; Birkhofer, H.: Linear guides of linear flow split components - Development and integration of potential additional functions. In: Chakrabarti, A. (Hrsg.): Proceedings of the $3^{\text {rd }}$ International Conference on Research into Design. ICoRD 2011. Bangalore, Indien. Singapur: 2011, S. 439-446.

\section{Lotter, Wiendahl (2006)}

Lotter, B.; Wiendahl, H.-P.: Montage in der industriellen Produktion. Ein Handbuch für die Praxis. Berlin - Heidelberg: Springer Verlag 2006. https://doi.org/10.1007/3540-36669-5.

\section{Ludwig et al. (2010)}

Ludwig, C.; Jalizi, B.; Apprich, S.; Abele, E.; Groche, P.: Integration von Fräs- und Spaltprofilierprozessen in Walzprofilieranlagen. In: Groche, P. (Hrsg.):

Tagungsband Sonderforschungsbereich 666, Integrale Blechbauweisen höherer Verzweigungsordnung - Entwicklung, Fertigung, Bewertung. 3.

Zwischenkolloquium. Vol. 3. Bamberg: 2010, S. 61-70.

\section{Lüthen et al. (2017)}

Lüthen, H.; Gramlich, S.; Horn, B.; Mattmann, I.; Pfetsch, M.; Roos, M.; Ulbrich, S.; Wagner, C.; Walter, A.: Finding the Best: Mathematical Optimization Based on Product and Process Requirements. In: Groche, P.; Bruder, E.; Gramlich, S. (Hrsg.): Manufacturing Integrated Design. Sheet Metal Product and Process Innovation. Cham: Springer International Publishing 2017, S. 147-200.

\section{Matek et al. (2011)}

Matek, W.; Roloff, H.; Wittel, H.; Muhs, D.; Jannasch, D.; Voßiek, J.: Roloff/Matek Maschinenelemente. 20. Aufl. Wiesbaden: Vieweg + Teubner 2011.

\section{Meerkamm et al. (2012)}

Meerkamm, H.; Wartzack, S.; Bauer, S.; Krehmer, H.; Stockinger, A.; Walter, M.: Design for X (DFX). In: Rieg, F.; Steinhilper, R. (Hrsg.): Handbuch Konstruktion. München - Wien: Hanser Verlag 2012, S. 443-462.

\section{Mertins et al. (2016)}

Mertins, K.; Kohl, l.; Orth, R.: Ein Referenzmodell für Wissensmanagement. In: Kohl, H.; Mertins, K.; Seidel, H. (Hrsg.): Wissensmanagement im Mittelstand. Berlin - Heidelberg: Springer Verlag 2016. 


\section{Monnerjahn, Fricke (2012)}

Monnerjahn, V.; Fricke, S.: Potenziale des Festwalzens zur Optimierung von

Oberflächen beim Walzprofilieren. In: Groche, P. (Hrsg.): 8. Fachtagung

Walzprofilieren und 4. Zwischenkolloquium des Sonderforschungsbereichs 666.

Darmstadt-Wixhausen. Bamberg: 2012, S. 125-141.

\section{Munirathnam et al. (2007)}

Munirathnam, M.; Vucic, D.; Abele, E.; Groche, P.: Flexible Fertigungsanlage zur Herstellung verzweigter Mehrkammerprofile. In: Groche, P. (Hrsg.): Tagungsband Sonderforschungsbereich 666, Integrale Blechbauweisen höherer Verzweigungsordnung - Entwicklung, Fertigung, Bewertung. 1.

Zwischenkolloquium. Vol. 1. Bamberg: 2007, S. 85-94.

\section{Neugebauer (2007)}

Neugebauer, R.: Hydro-Umformung. Berlin u. a.: Springer Verlag 2007.

\section{Neuwirth et al. (2017)}

Neuwirth, M.; Abedini, S.; Abele, E.; Groche, P.; Köhler, S.; Monnerjahn, V.;

Schäfer, S.; Schmidt, S.; Turan, E.: New Technologies: From Basic Ideas to Mature Technologies. In: Groche, P.; Bruder, E.; Gramlich, S. (Hrsg.): Manufacturing Integrated Design. Sheet Metal Product and Process Innovation. Cham: Springer International Publishing 2017, S. 31-97.

\section{Niehuesbernd et al. (2013)}

Niehuesbernd, J.; Müller, C.; Pantleon, W.; Bruder, E.: Quantification of local and global elastic anisotropy in ultrafine grained gradient microstructures, produced by linear flow splitting. In: Materials Science and Engineering: A, 560. 2013, S. 273277. https://doi.org/10.1016/j.msea.2012.09.067.

\section{Noack, Sterzing (2007)}

Noack, S.; Sterzing, A.: Grundlagen. In: Neugebauer, R. (Hrsg.): HydroUmformung. Berlin u. a.: Springer Verlag 2007, S. 15-32.

\section{Oberender (2006)}

Oberender, C.: Die Nutzungsphase und ihre Bedeutung für die Entwicklung umweltgerechter Produkte. Fortschr.-Ber. VDI Reihe 1, Nr. 385. Düsseldorf: VDIVerlag 2006. Dissertation. 
Pahl et al. (2007)

Pahl, G.; Beitz, W.; Feldhusen, J.; Grote, K.-H.: Konstruktionslehre. Grundlagen erfolgreicher Produktentwicklung Methoden und Anwendung. 7. Aufl. Berlin Heidelberg: Springer Verlag 2007.

Poli (2001)

Poli, C.: Design for manufacturing. A structured approach. Boston: ButterworthHeinemann 2001.

\section{Ponn, Lindemann (2008)}

Ponn, J.; Lindemann, U.: Konzeptentwicklung und Gestaltung technischer Produkte. Optimierte Produkte - systematisch von Anforderungen zu Konzepten. Berlin - Heidelberg: Springer Verlag 2008.

\section{Probst et al. (2012)}

Probst, G.; Raub, S.; Romhardt, K.: Wissen managen. Wie Unternehmen ihre wertvollste Ressource optimal nutzen. 7. Auflage. Wiesbaden: Springer Gabler 2012.

\section{Pröbster (2013)}

Pröbster, M.: Elastisch Kleben. Aus der Praxis für die Praxis. Wiesbaden: Springer Vieweg 2013.

\section{Rehäuser, Krcmar (1996)}

Rehäuser, J.; Krcmar, H.: Wissensmanagement im Unternehmen. Arbeitspapiere, Lehrstuhl für Wirtschaftsinformatik, Universität Hohenheim, Nr. 98. Stuttgart: 1996.

\section{Ringler, Groche (2008)}

Ringler, J.; Groche, P.: Spaltbiegen - Ein neues Verfahren für integrale

Verzweigungen aus der Blechmitte. In: Groche, P. (Hrsg.): Tagungsband

Sonderforschungsbereich 666, Integrale Blechbauweisen höherer

Verzweigungsordnung - Entwicklung, Fertigung, Bewertung. 2.

Zwischenkolloquium. Vol. 2. Bamberg: 2008, S. 63-71.

\section{Rodenacker (1991)}

Rodenacker, W. G.: Methodisches Konstruieren. Grundlagen, Methodik, praktische Beispiele. Vierte, überarb. Aufl. Konstruktionsbücher, Nr. 27. Berlin - Heidelberg: Springer Verlag 1991. 
Röder et al. (2012)

Röder, B.; Gramlich, S.; Birkhofer, H.: Von der abstrakten Anforderung zur formalisierten Entwicklungsaufgabe. In: Groche, P. (Hrsg.): Tagungsband Sonderforschungsbereich 666, Integrale Blechbauweisen höherer Verzweigungsordnung - Entwicklung, Fertigung, Bewertung. 4. Zwischenkolloquium. Vol. 4. Bamberg: 2012, S. 5-14.

\section{Rollmann (2012)}

Rollmann, T.: Simultaneous engineering von integralen Blechbauweisen höherer Verzweigungsordnung - Ein Beitrag zur Integration von Konstruktion und Produktionsprozessplanung. Forschungsberichte aus dem Fachgebiet Datenverarbeitung in der Konstruktion, Nr. 40. Aachen: Shaker Verlag 2012. Dissertation.

\section{Roos et al. (2014)}

Roos, M.; Gramlich, S.; Kloberdanz, H.; Ćorić, M.: Using Manufacturing Technological Potential in Product Design - A Cognition-Based Approach. In: Marjanović, D.; Štorga, M.; Pavković, N.; Bojčetić, N. (Hrsg.): Proceedings of the $13^{\text {th }}$ International Design Conference. DESIGN 2014. Dubrovnik, Kroatien. Zagreb: 2014, S. 1853-1862.

\section{Roos et al. (2016)}

Roos, M.; Horn, B.; Gramlich, S.; Ulbrich, S.; Kloberdanz, H.: Manufacturing Integrated Algorithm-Based Product Design - Case Study of a Snap-Fit Fastening. In: Procedia CIRP, 50. 2016, S. 123-128.

https://doi.org/10.1016/j.procir.2016.04.195.

\section{Roos et al. (2017a)}

Roos, M.; Wagner, C.; Weber Martins, T.; Albrecht, K.; Anderl, R.; Kirchner, E.: Manufacturing-integrated product solutions. Design support between product function and manufacturing processes. In: Materialwissenschaft und Werkstofftechnik, 48 (1). 2017, S. 12-26. https://doi.org/10.1002/mawe.201600727.

\section{Roos et al. (2017b)}

Roos, M.; Wagner, C.; Gramlich, S.; Reichwein, J.; Kirchner, E.: Selective Pre-load Generation: Finding Manufacturing-integrated Solutions for Linear Guides. In: Maier, A.; Škec, S.; Kim, H.; Kokkolaras, M.; Oehmen, J.; Fadel, G.; Salustri, F.; Van der Loos, M. (Hrsg.): Proceedings of the $21^{\text {st }}$ International Conference on Engineering Design. ICED 2017. Vol. 1. Vancouver, Canada: 2017, S. 269-278. 


\section{Roos et al. (2017c)}

Roos, M.; Abedini, S.; Abele, E.; Albrecht, K.; Anderl, R.; Gibbels, M.; Gramlich, S.; Groche, P.; Horn, B.; Hoßfeld, A.; Köhler, S.; Lüthen, H.; Mattmann, I.; Melz, T.; Monnerjahn, V.; Müller, C.; Neuwirth, M.; Niehuesbernd, J.; Özel, M.; Pfetsch, M.; Reising, J.; Schäfer, S.; Schmidt, S.; Turan, E.; Ulbrich, S.; Wagner, C.; Walter, A.; Weber Martins, T.; Zimmermann, A.: The Result: A New Design Paradigm. In: Groche, P.; Bruder, E.; Gramlich, S. (Hrsg.): Manufacturing Integrated Design. Sheet Metal Product and Process Innovation. Cham: Springer International Publishing 2017, S. 301-334.

\section{Ropohl (2009)}

Ropohl, G.: Allgemeine Technologie. Eine Systemtheorie der Technik. 3., überarb. Aufl. Karlsruhe: Universitätsverlag Karlsruhe 2009.

\section{Roth (2000)}

Roth, K.: Konstruieren mit Konstruktionskatalogen. Konstruktionslehre. 3. Aufl., erw. und neu gestaltet. Konstruieren mit Konstruktionskatalogen, Nr. 1. Berlin Heidelberg: Springer Verlag 2000.

\section{Roth (2001)}

Roth, K.: Konstruieren mit Konstruktionskatalogen. Kataloge. 3. Aufl., mit wesentl. Erg. Konstruieren mit Konstruktionskatalogen, Nr. 2. Berlin u. a.: Springer Verlag 2001.

\section{Sauer (2006)}

Sauer, T.: Ein Konzept zur Nutzung von Lösungsobjekten für die

Produktentwicklung in Lern- und Anwendungssystemen. Fortschr.-Ber. VDI Reihe 1, Nr. 390. Düsseldorf: VDI-Verlag 2006. Dissertation.

\section{Schönherr (2002)}

Schönherr, H.: Spanende Fertigung. München - Wien: Oldenbourg Verlag 2002.

\section{Schott (1998)}

Schott, H.: Informationsressourcen und Informationsmanagement für die Entwicklung umweltgerechter Produkte. Fortschr.-Ber. VDI Reihe 1, Nr. 297. Düsseldorf: VDI-Verlag 1998. Dissertation.

\section{Seidel, Hahn (2010)}

Seidel, W. W.; Hahn, F.: Werkstofftechnik. Werkstoffe - Eigenschaften - Prüfung Anwendung. 8., neu bearb. Aufl. Lernbücher der Technik. München: Hanser Verlag 2010. 


\section{Suh (1998)}

Suh, N. P.: Axiomatic Design Theory for Systems. In: Research in Engineering Design, 10 (4). 1998, S. 189-209. https://doi.org/10.1007/s001639870001.

\section{Tangen (2005)}

Tangen, S.: Demystifying productivity and performance. In: International Journal of Productivity and Performance Management, 54 (1). 2005, S. 34-46.

https://doi.org/10.1108/17410400510571437.

\section{Tekkaya et al. (2015)}

Tekkaya, A. E.; Allwood, J. M.; Bariani, P. F.; Bruschi, S.; Cao, J.; Gramlich, S.;

Groche, P.; Hirt, G.; Ishikawa, T.; Löbbe, C.; Lueg-Althoff, J.; Merklein, M.; Misiolek, W. Z.; Pietrzyk, M.; Shivpuri, R.; Yanagimoto, J.: Metal forming beyond shaping.

Predicting and setting product properties. In: CIRP Annals - Manufacturing Technology, 64 (2). 2015, S. 629-653. https://doi.org/10.1016/j.cirp.2015.05.001.

\section{Tröster (2005)}

Tröster, F.: Steuerungs- und Regelungstechnik für Ingenieure. 2., überarb. und enw. Aufl. Oldenbourg Lehrbücher für Ingenieure. München - Wien: Oldenbourg Verlag 2005.

\section{Ulrich, Eppinger (2008)}

Ulrich, K. T.; Eppinger, S. D.: Product Design and Development. 4th ed. Boston u. a.: McGraw-Hill 2008.

\section{VDI 2206 (2004)}

VDI 2206:2004-06, Entwicklungsmethodik für mechatronische Systeme. Verein Deutscher Ingenieure (Hrsg.). Berlin: Beuth Verlag 2004.

\section{VDI 2221 (1993)}

VDI 2221:1993-05, Methodik zum Entwickeln und Konstruieren technischer Systeme und Produkte. Verein Deutscher Ingenieure (Hrsg.). Berlin: Beuth Verlag 1993.

\section{VDI 2223 (2004)}

VDI 2223:2004-01, Methodisches Entwerfen technischer Produkte. Verein

Deutscher Ingenieure (Hrsg.). Berlin: Beuth Verlag 2004.

\section{VDI 2225-3 (1998)}

VDI 2225-3:1998-11, Konstruktionsmethodik - Technisch-wirtschaftliches Konstruieren - Technisch-wirtschaftliche Bewertung. Verein Deutscher Ingenieure (Hrsg.). Berlin: Beuth Verlag 1998. 


\section{VDI 2860 (1990)}

VDI 2860:1990-05, Montage- und Handhabungstechnik; Handhabungsfunktionen, Handhabungseinrichtungen; Begriffe, Definitionen, Symbole. VDI-Gesellschaft Produktion und Logistik (Hrsg.). Berlin: Beuth Verlag 1990.

\section{VDI 3146-1 (1999)}

VDI 3146-1:1999-03 (Entwurf): Innenhochdruck-Umformen - Grundlagen. Verein Deutscher Ingenieure (Hrsg.). Düsseldorf: Beuth Verlag 1999.

\section{Wäldele (2012)}

Wäldele, M.: Erarbeitung einer Theorie der Eigenschaften technischer Produkte. Ein Beitrag für die konventionelle und algorithmenbasierte Produktentwicklung. Fortschr.-Ber. VDI Reihe 1, Nr. 414. Düsseldorf: VDI-Verlag 2012. Dissertation.

\section{Wagner et al. (2014)}

Wagner, C.; Gramlich, S.; Kloberdanz, H.: Entwicklung innovativer Produkte durch Verknüpfung von Funktionsintegration und Fertigungsprozessintegration. In:

Krause, D.; Paetzold, K.; Wartzack, S. (Hrsg.): Proceedings of the 24 $4^{\text {th }}$ Symposium on Design for X. DfX 2014. Bamberg: 2014, S. 361-372.

\section{Wagner et al. (2016)}

Wagner, C.; Roos, M.; Gramlich, S.; Kloberdanz, H.: Process Integrated Design Guidelines - Systematically Linking Manufacturing Processes to Product Design. In: Marjanovic, D.; Storga, M.; Pavkovic, N.; Bojcetic, N.; Skec, S. (Hrsg.): Proceedings of the $14^{\text {th }}$ International Design Conference. DESIGN 2016. Dubrovnik, Kroatien: 2016, S. 739-748.

\section{Wagner et al. (2017)}

Wagner, C.; Ahmels, L.; Gramlich, S.; Groche, P.; Monnerjahn, V.; Müller, C.; Roos, M.: Finding New Opportunities: Technology Push Approach. In: Groche, P.; Bruder, E.; Gramlich, S. (Hrsg.): Manufacturing Integrated Design. Sheet Metal Product and Process Innovation. Cham: Springer International Publishing 2017, S. 275-299.

\section{Weber (2005)}

Weber, C.: CPM/PDD - An extended theoretical approach to modelling products and products development processes. In: Proceedings of the $3^{\text {rd }}$ International PhD Conference on Mechanical Engineering. PhD 2005. Srni, Tschechische Republik. Pilsen: 2005, S. 11-28. 


\section{Weber (2012)}

Weber, C.: Produkte und Produktentwicklungsprozesse abbilden mit Hilfe von Merkmalen und Eigenschaften - eine kritische Zwischenbilanz. In: Krause, D.; Paetzold, K.; Wartzack, S. (Hrsg.): Proceedings of the $23^{\text {rd }}$ Symposium on Design for X. DfX 2012. Bamberg/Erlangen, Deutschland: 2012, S. 25-62.

\section{Weber, Werner (2000)}

Weber, C.; Werner, H.: Klassifizierung von CAx-Werkzeugen für die Produktentwicklung auf der Basis eines neuartigen Produkt- und Prozessmodells. In: Meerkamm, H. (Hrsg.): Proceedings of the $11^{\text {th }}$ Symposium on Design for X. DfX 2000. Schnaittach/Erlangen, Deutschland: 2000, S. 126-143.

\section{Weigand et al. (2010)}

Weigand, B.; Köhler, J.; Wolfersdorf, J.: Thermodynamik kompakt. SpringerLehrbuch. Berlin - Heidelberg: Springer Verlag 2010.

\section{Westkämper (2006)}

Westkämper, E.: Einführung in die Organisation der Produktion. Springer-Lehrbuch. Berlin, Heidelberg: Springer 2006. https://doi.org/10.1007/3-540-30764-8.

\section{Westkämper, Warnecke (2010)}

Westkämper, E.; Warnecke, H.-J.: Einführung in die Fertigungstechnik. Wiesbaden: Vieweg+Teubner 2010. https://doi.org/10.1007/978-3-8348-9798-5.

\section{Zangemeister (1976)}

Zangemeister, C.: Nutzwertanalyse in der Systemtechnik. Eine Methodik zur multidimensionalen Bewertung und Auswahl von Projektalternativen. 4. Aufl. Hamburg: Zangemeister 1976. 


\section{Betreute studentische Arbeiten}

\section{4}

Eller, Dominik; Griesel, Dominic; Gute, Heike; Hau, Raphael René: Konstruktion eines Linearsystems mit spaltprofilierten Führungsflächen und integriertem LinearmotorAntrieb. Advanced Design Project, 2014.

Gögelein, Eva: Die Nutzung fertigungstechnologischen Wissens im Rahmen von Knowledge Based Engineering und konventionellen PE-Ansätzen. BachelorThesis, 2014.

Mattmann, llyas: Transformation von Marktanforderungen in Produkteigenschaften. Master-Thesis, 2014.

Reichwein, Jannik: Analytische Untersuchung von Verschleiß und Versagen bei spaltprofilierten Bauteilen am Beispiel von Schnappverbindungen. Bachelor-Thesis, 2014.

\section{5}

Camborda, Juan Carlos; Döring, Hans Christian; Eller, Dominik; Gute, Heike; Köppen, Christopher; Vogel, Sven: Systematische Integration fertigungstechnologischer Aspekte in die Produktentwicklung. Forschungsseminar, 2015.

Süßenguth, Jan: Untersuchung und Systematisierung relevanter Einflussgrößen auf die Tragfähigkeit von Passfederverbindungen. Bachelor-Thesis, 2015.

Walle, Ernst-Ludwig: Adaption von Wissensmanagementprozessen zur Strukturierung einer fertigungsintegrierenden Produktentwicklung. Master-Thesis, 2015.

\section{6}

Listmann, Victor: Entwicklung einer innovativen spaltprofilierten Schnappverbindung. Bachelor-Thesis, 2016. 


\section{Index}

\section{A}

Antizipieren techn. Prozesse ...39, 80

D

Design for $\mathrm{X}$ 14

Design for Assembly 15

Design for Manufacture 15

Design for Manufacture and Assembly 15

Design Pattern 89,103

Design Pattern Matrix 91, 102, 116

\section{E}

Eigenschaft 21

Ausprägung................................. 21

der Oberfläche ............................ 23

Eigenschaftsnetzwerk .................25

Eigenschaftsrelationen...............25

Gestalteigenschaft ..................... 22

mechanische ............................. 22

Merkmal ...................................... 21

physikalische.............................. 23

Produkteigenschaft abhängige ................................ 21

unabhängige 21

Produktmodelleigenschaft abhängige 22

unabhängige 22

Prozesseigenschaft 60

verfahrensinduzierte $35,40,52,82$ verfahrensprinziprelevante... 35,59 verfahrensrelevante 51,59 werkstoffliche .. 22

\section{$\boldsymbol{F}$}

Fertigung......................................... 1

Fertigungstechnik....................... 8

Fertigungstechnologie..............2, 8 fertigungsintegrierende

Entwicklungsmethodik 83,85 Lösungselemente.....45, 88, 93, 95

Produktlösung $6,45,75$ Fügen 38

Funktionsträger $26,91,100$

G

Ganzheitliche Produkt- und

Prozessentwicklung .......39, 46, 76

Gestalt 22

Gestaltung

fertigungsgerecht ...................... 13

montagegerecht .......................... 13

Gestaltungsrichtlinie ..................... 13

Gestaltvorbild ................................. 13

Größe 19,25

Funktionsgröße 19

Prozessgröße. 20

Wirkgröße 28,73

Zustandsgröße 20

I

Innenhochdruckumformen .. 3, 11, 53, $55,61,100$

M

Mapping 41

Methode 17

Methodik 17

Modell 
Gestaltmodell .............................. 20

Produktmodell .............................. 18

Produktmodellpyramide

Prozessmodell .18

Verfahrensprinzipmodell 58,81

Vorgehensmodell 38

Montieren. 38

\section{N}

Nachbarsystem 59

0

Objekt

\section{$\boldsymbol{P}$}

Physikalischer Effekt 20,55

Process Integrated Design

Guideline 95

Produktion 1

produktionsintegrierende

Entwicklungsmethodik ... 40, 46, 76

Produktlebenslauf.

37,39

$S$

Schnappverbindung 111,117

Spaltprofilieren..... 2, 4, 9, 55, 64, 111

\section{$T$}

technischer Prozess 28

Anfangszustand .28

Arbeitsmittel .28

Einsatzprozess 37,67

Endzustand .. 28

fertigungsanaloger

Nutzungsprozess 37

Fügeprozesse 113

Operand 27,52

Prozesskette
Prozessstruktur 30,53

Transformationsprozess 27

Zustand 34

Zustandsänderung 28,50 technisches Produkt 17

Konkretisierung 18,39

Physikalischer Effekt 20

Produktfunktion 19,73

Gesamtfunktion 20

Teilfunktion 20

Produktgestalt 22

Wirkprinzip 20

V

Verfahren $8,30,32$

Fertigungsverfahren 47

Physikalischer Effekt .55

-sinduziertes Gestaltelement .... 35, 51,91

-srelevantes Gestaltelement ...... 51

Verfahrenskette. 54

Verfahrensprinzip $32,48,57$

-relevante Wirkgröße .59 -relevantes Nachbarelement .. 59 -relevantes Wirkelement.......... 33 -relevantes Wirkelement 59

W

Wirkflächenpaar 114 Wissen

fertigungstechn. Produkt-Wissen $49,57,74,77$ fertigungstechn. Wissen.. 1, 47, 74, 75

Produkt-Wissen 48 